NUREG/CR-7024

PNNL-19417

\title{
Material Property Correlations: Comparisons between FRAPCON-3.4, FRAPTRAN 1.4, and MATPRO
}

Manuscript Completed: August 2010

Date Published: August 2010

Prepared by

W.G. Luscher and K.J. Geelhood

Pacific Northwest National Laboratory

Richland, WA 99352

Prepared for

Division of System Analysis

Office of Nuclear Regulatory Research

U.S. Nuclear Regulatory Commission

Washington DC 20555-0001

NRC Job Code N6609 



\begin{abstract}
The U.S. Nuclear Regulatory Commission uses the computer codes FRAPCON-3 and FRAPTRAN to model steady-state and transient fuel behavior, respectively, in regulatory analysis. In this document, material property correlations for oxide fuels and cladding materials are presented and discussed. Comparisons are made between the material property correlations used in the most recent versions of the codes, FRAPCON-3.4 and FRAPTRAN 1.4. Comparisons are also made with MATPRO, which is a compilation of material property correlations with an extensive history of use with various fuel performance and severe accident codes. In addition to model-to-model comparisons, model-to-data comparisons and source codes for the FRAPCON-3.4 and FRAPTRAN 1.4 correlations are provided.
\end{abstract}




\section{Foreword}

The U.S. Nuclear Regulatory Commission uses the computer codes FRAPCON and FRAPTRAN to model steady-state and transient fuel behavior, respectively, in regulatory analysis. To effectively model fuel behavior, material property correlations must be used for a wide range of operating conditions (e.g., temperature and burnup). In this sense, a "material property" is a physical characteristic of the material whose quantitative value is necessary in the analysis process.

The consolidated resource for "material properties" cited most often in the literature is MATPRO (as documented in Volume 4 of NUREG/CR-6150). MATPRO is a compilation of fuel and cladding material property correlations with an extensive history of use with fuel performance and severe accident codes. However, consistency among the material property correlations in FRAPCON-3, FRAPTRAN, and MATPRO has never been complete, as the material property correlations in the fuel performance codes have evolved to accommodate recent fuel design changes.

The primary purpose of this report is to consolidate the current material property correlations used in FRAPCON and FRAPTRAN into a single document. In addition to describing the material property correlations used in the subroutines of latest versions of the codes, FRAPCON3.4 and FRAPTRAN 1.4, this report also provides a variety of comparisons to the material property correlations used in MATPRO. 


\section{Contents}

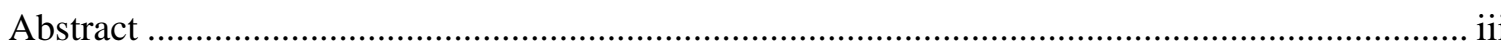

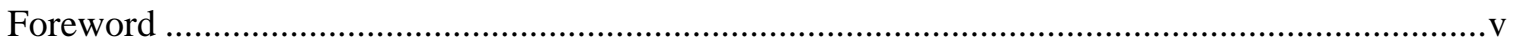

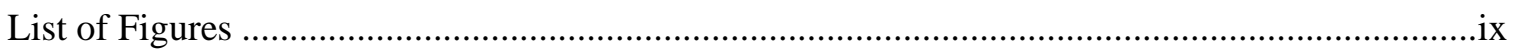

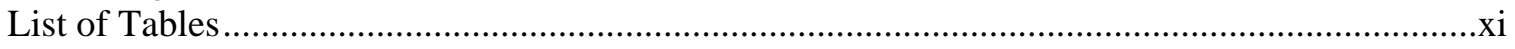

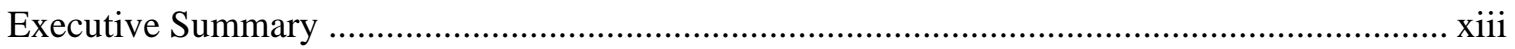

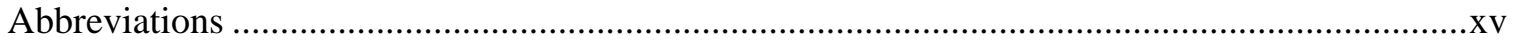

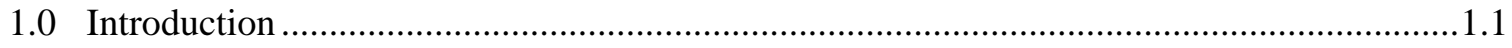

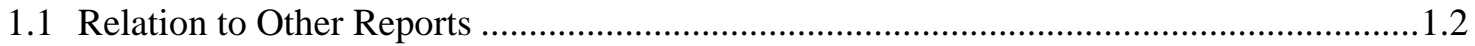

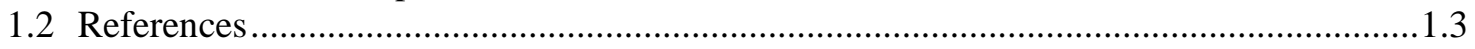

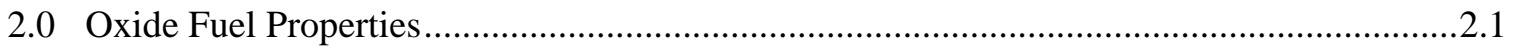

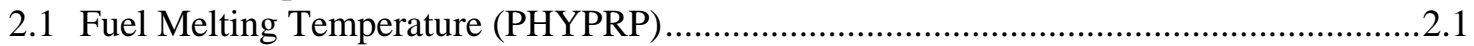

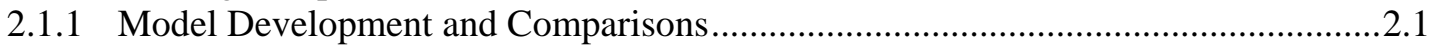

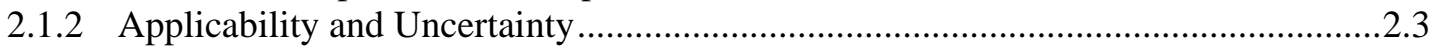

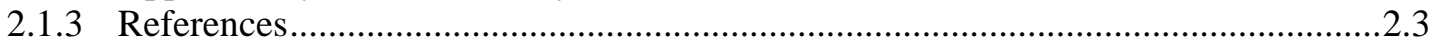

2.2 Fuel Specific Heat Capacity (FCP) and Fuel Enthalpy (FENTHL) ..................................2.4

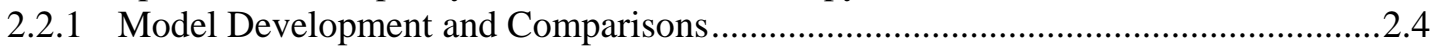

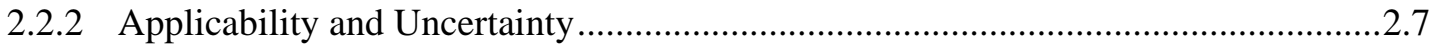

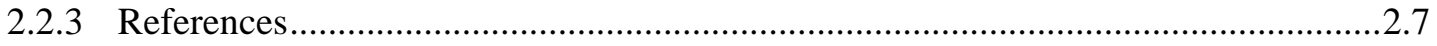

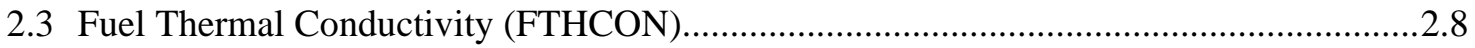

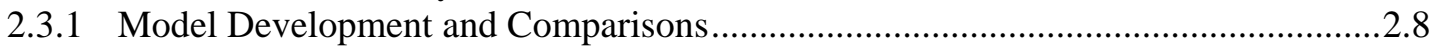

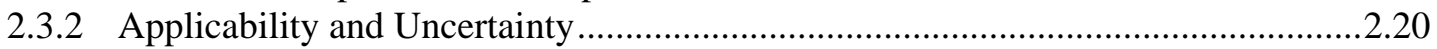

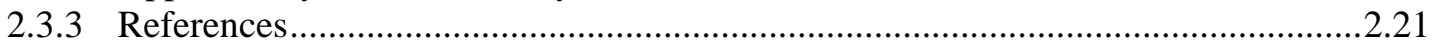

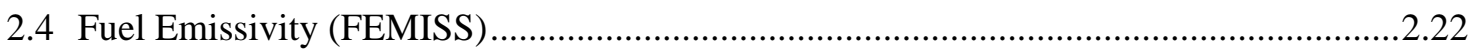

2.4.1 Model Development and Comparisons......................................................................2.2.22

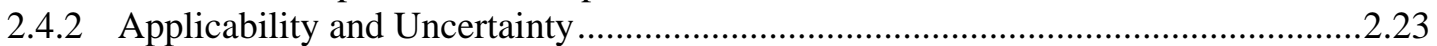

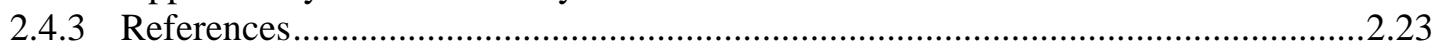

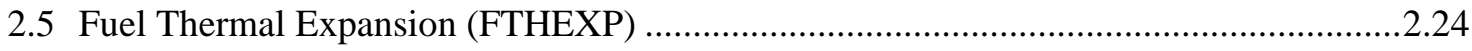

2.5.1 Model Development and Comparisons..................................................................2.24

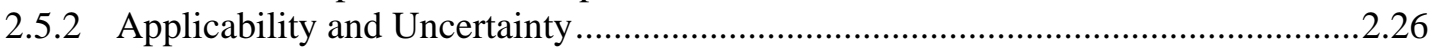

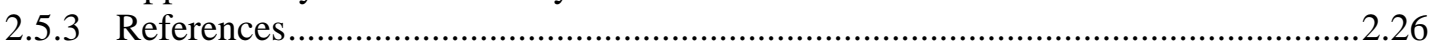

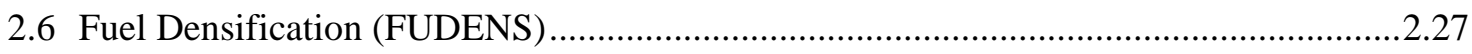

2.6.1 Model Development and Comparisons................................................................2.27

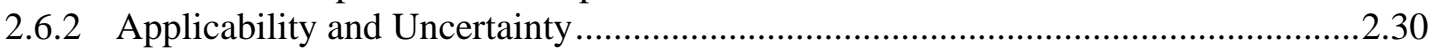

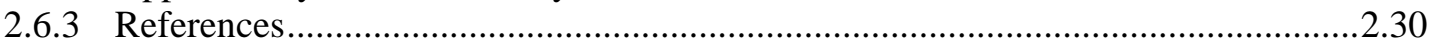

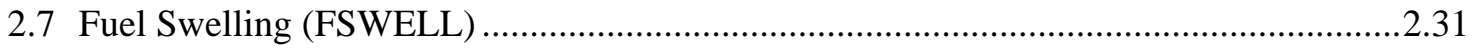

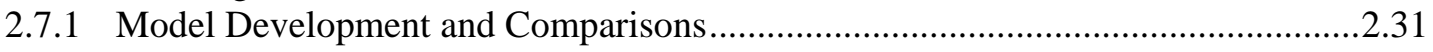

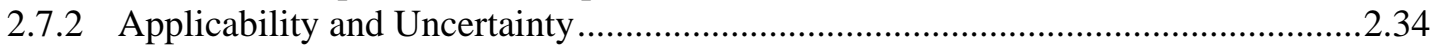

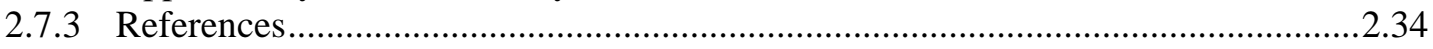

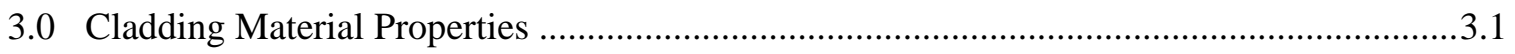

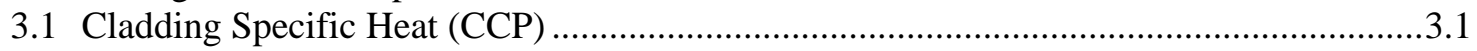

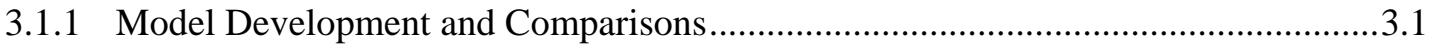

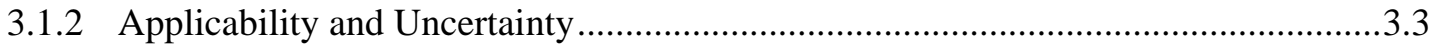

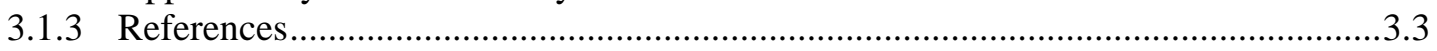

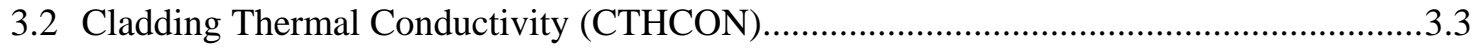

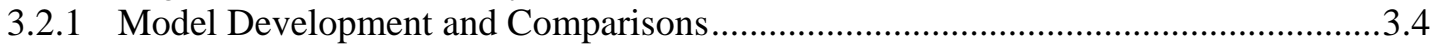

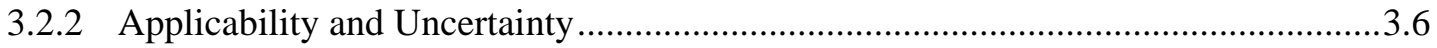

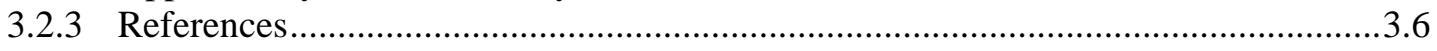

3.3 Cladding Oxide Thermal Conductivity (ZOTCON) ......................................................... 


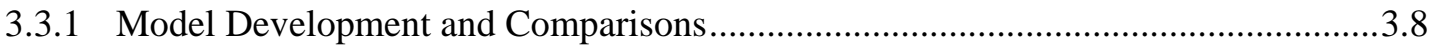

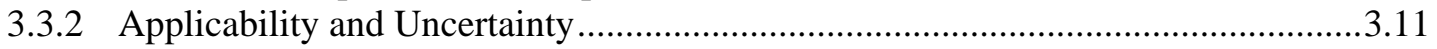

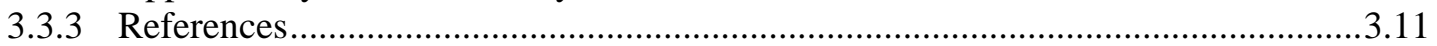

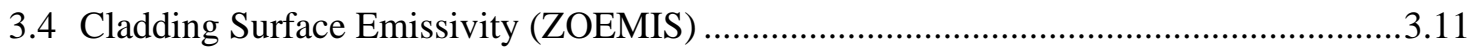

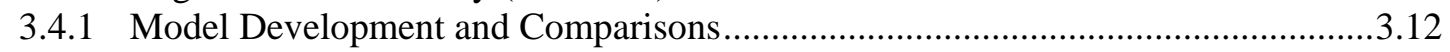

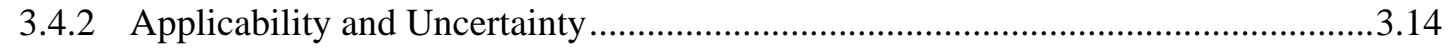

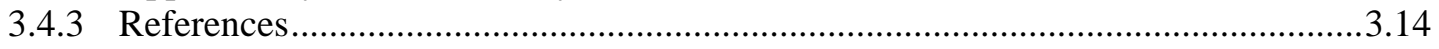

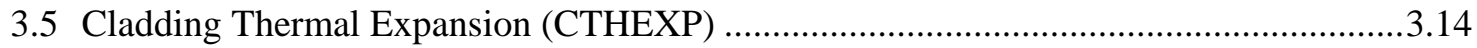

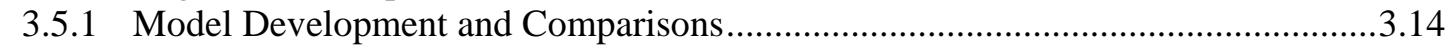

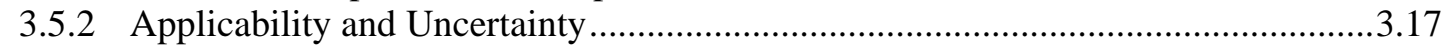

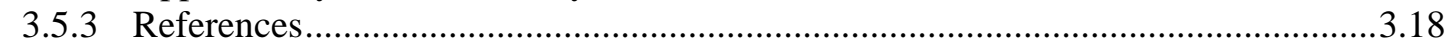

3.6 Cladding Elastic Modulus (CELMOD) and Shear Modulus (CSHEAR) ..........................18

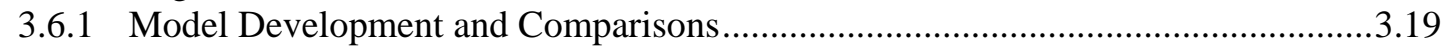

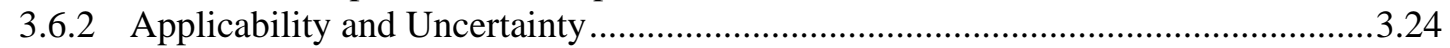

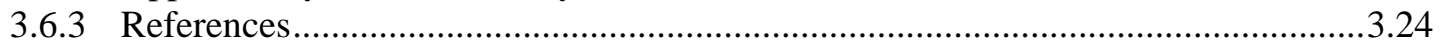

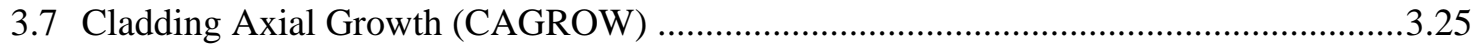

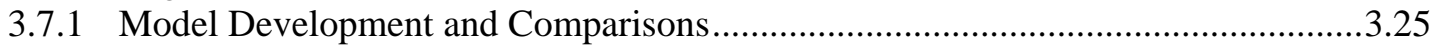

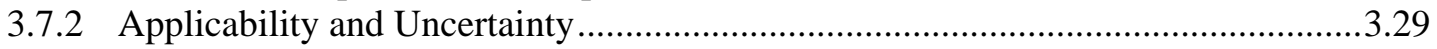

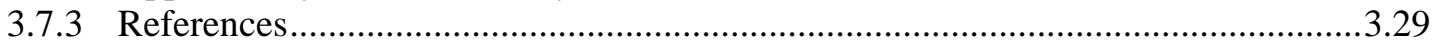

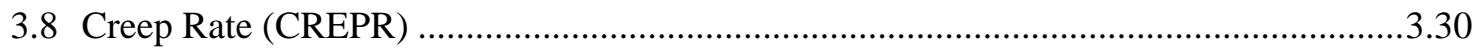

3.8.1 Model Development and Comparisons..............................................................3.30

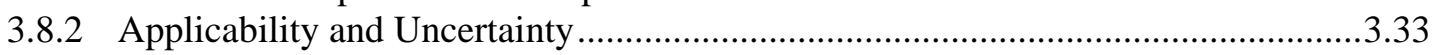

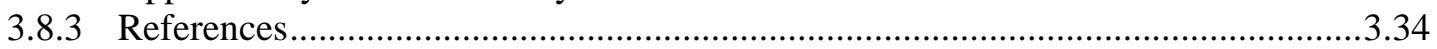

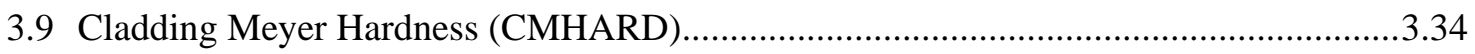

3.9.1 Model Development and Comparisons.................................................................3.35

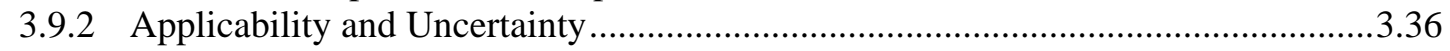

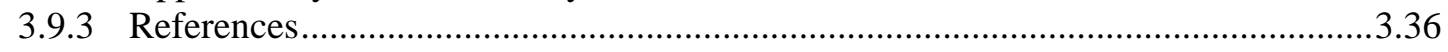

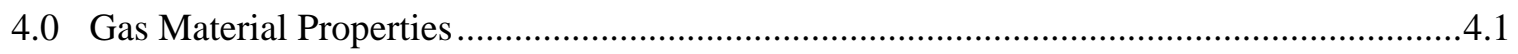

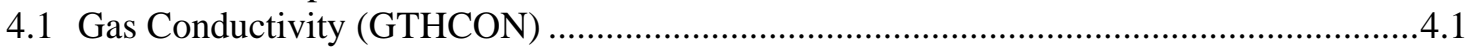

4.1.1 Model Development and Comparisons................................................................. 4.1

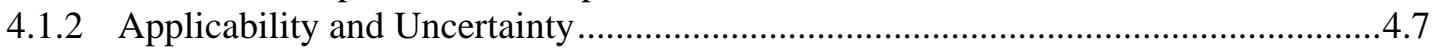

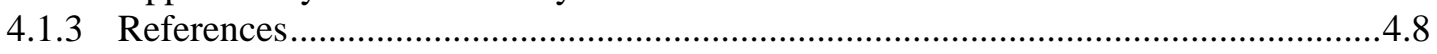

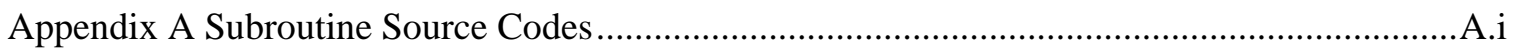




\section{List of Figures}

Figure 2.1-1 A comparison between solidus temperatures calculated by MATPRO using the FHYPRP subroutine and FRAPCON-3/FRAPTRAN (FRAP) using the PHYPRP subroutine. Results for burnup varying from 0 to $62 \mathrm{GWd} / \mathrm{MTU}$ and Pu contents of 0,3 , and $7 \mathrm{wt} \%$ are presented. 2.2

Figure 2.1-2 A comparison between liquidus temperatures calculated by MATPRO using the FHYPRP subroutine and FRAPCON-3/FRAPTRAN (FRAP) using the PHYPRP subroutine. Results for burnup varying from 0 to $62 \mathrm{GWd} / \mathrm{MTU}$ and $\mathrm{Pu}$ contents of 0,3 , and $7 \mathrm{wt} \%$ are presented. 2.3

Figure 2.2-1 Specific heat capacity of $\mathrm{UO}_{2}$ from three experimenters compared with the FRAPCON-3/FRAPTRAN/MATPRO (FRAP) correlation (solid line) for $\mathrm{UO}_{2} .2 .6$

Figure 2.2-2 Specific heat capacity of $\left(\mathrm{U}_{0.8} \mathrm{Pu}_{0.2}\right) \mathrm{O}_{2}$ from three experimenters compared with the FRAPCON-3/FRAPTRAN/MATPRO (FRAP) correlations (solid line) for MOX.

Figure 2.3.3-1 MATPRO and modified NFI thermal conductivity models for $\mathrm{UO}_{2}$ as a function of temperature. Unlike the MATPRO model, the modified NFI model can account for burnup and is presented at burnup levels of 0 and $30 \mathrm{GWd} / \mathrm{MTU}$ for comparison. 2.14

Figure 2.3.3-2 MATPRO model-to-data comparison for thermal conductivity of unirradiated

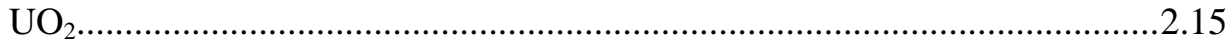

Figure 2.3.3-3 Modified NFI model-to-data comparison for thermal conductivity of unirradiated $\mathrm{UO}_{2}$. 2.15

Figure 2.3.3-4 MATPRO model-to-data comparison for thermal conductivity of irradiated

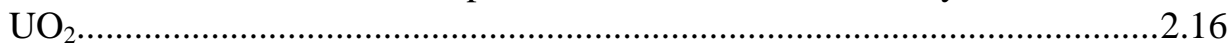

Figure 2.3.3-5 Modified NFI model-to-data comparison for thermal conductivity of irradiated

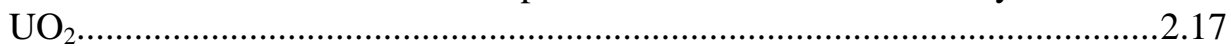

Figure 2.3.3-6 MATPRO and Duriez/modified NFI thermal conductivity models as a function of temperature for MOX fuel with $7 \mathrm{wt} \%$ plutonium content. Unlike the MATPRO model, the Duriez/modified NFI model can account for burnup and is presented at burnup levels of 0 and $30 \mathrm{GWd} / \mathrm{MTU}$ for comparison..........2.18

Figure 2.3.3-7 MATPRO model-to-data comparison for thermal conductivity of MOX modeled with $7 \mathrm{wt} \%$ of plutonium..............................................................2.19

Figure 2.3.3-8 Duriez/modified NFI model-to-data comparison for thermal conductivity of MOX with modeled with $7 \mathrm{wt} \%$ of plutonium. ............................................2.19

Figure 2.4-1 Data to model comparison for FEMISS........................................................2.23

Figure 2.5-1 Model-to-data comparison for $\mathrm{UO}_{2}$ correlation used in MATPRO and FRAPCON-3/FRAPTRAN with updated constants. ....................................2.25

Figure 2.5-2 Model-to-data comparison for $\mathrm{PuO}_{2}$ correlation used in MATPRO and FRAPCON-3/FRAPTRAN....................................................................2.26

Figure 2.6-1 Comparison of densification predictions generated by the FUDENS model and experimental densification measurements determined in reactor. ..................2.30

Figure 2.7-1 Data comparison between MATPRO and FRAPCON-3.4 versions of

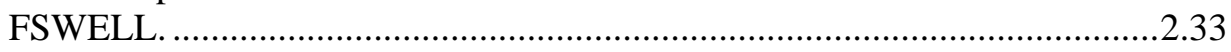

Figure 2.7-2 Comparison between swelling rates determined for various instrumented fuel assemblies tests at Halden and swelling rates predicted by FRAPCON-3.4..2.34

Figure 3.2-1 CTHCON model and data used for development. .3 .5

Figure 3.2-2

CTHCON model and data collected since development..... 3.6 
Figure 3.3-1 Oxide thermal conductivity measurements as a function of temperature compared with the MATPRO and FRAPCON-3.4/FRAPTRAN 1.4 (FRAP)

cladding oxide thermal conductivity models. .................................................3.10

Figure 3.4-1 Model-to-data comparison for cladding oxide emissivity................................13

Figure 3.5-1 Model-to-data comparison for FRAPCON-3.4/FRAPTRAN 1.4 (FRAP) and

MATPRO axial thermal expansion. Data from cladding tubes.......................3.17

Figure 3.5-2 Model-to-data comparison for FRAPCON-3.4/FRAPTRAN 1.4 (FRAP) and

MATPRO diametral thermal expansion. Data from cladding tubes. .............3.17

Figure 3.6-1 Predicted vs. measured values for Young's modulus for various data sets. ..3.23

Figure 3.7-5 Model-to-data comparison for SRA Zircaloy-4 (PWR). The standard error of

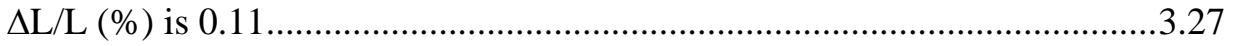

Figure 3.7-6 Model-to-data comparison for RXA Zircaloy-2 (BWR). The standard error of

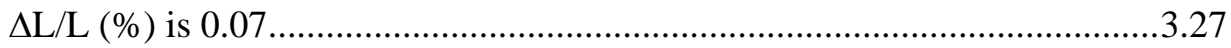

Figure 3.7-7 Model-to-data comparison for M5. M5 data was collected by Gilbon et al.

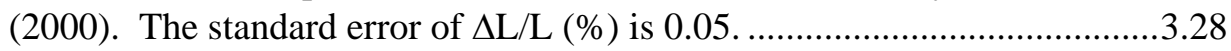

Figure 3.7-8 Model-to-data comparison for ZIRLO. Vandellos data was collected by Irisa (2000) while North Anna and BR-3 data was collected by Sabol (1994). The

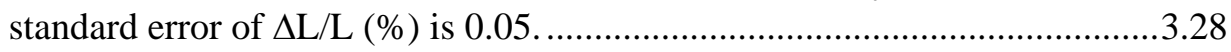

Figure 3.8-1 Predicted vs. measured hoop strain for SRA Zircaloy model..........................3.33

Figure 3.8-2 Predicted vs. measured hoop strain for RXA Zircaloy model. ......................3.33

Figure 3.9-1 CMHARD correlation as a function of temperature with data from Peggs and

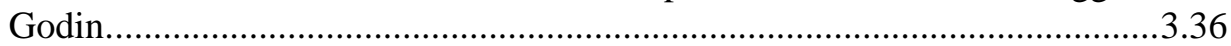

Figure 4.1-1 Model-to-data comparison for He thermal conductivity..................................4.3

Figure 4.1-2 Model-to-data comparison for Ar thermal conductivity. ..................................4.4

Figure 4.1-3 Model-to-data comparison for $\mathrm{Kr}$ thermal conductivity. ...................................5

Figure 4.1-4 Model-to-data comparison for Xe thermal conductivity...................................4.6

Figure 4.1-5 Model-to-data comparison for $\mathrm{H}_{2}$ thermal conductivity. ...............................4.6

Figure 4.1-6 Model-to-data comparison for $\mathrm{N}_{2}$ thermal conductivity.................................

Figure 4.1-7 Model-to-data comparison for steam thermal conductivity. .............................4.7 


\section{List of Tables}

Table 1.1 Roadmap to documentation of models and properties in NRC fuel performance codes, FRAPCON-3.4 and FRAPTRAN 1.4 ...............................................1.2

Table 2.2-1 Constants used in $\mathrm{UO}_{2}, \mathrm{Gd}_{2} \mathrm{O}_{3}$, and $\mathrm{PuO}_{2}$ heat capacity and enthalpy

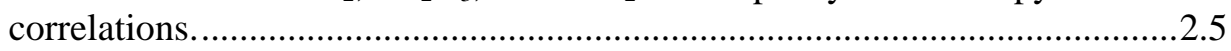

Table 2.5-1 Parameters used in $\mathrm{UO}_{2}$ and $\mathrm{PuO}_{2}$ solid-phase thermal expansion correlations.2.24

Table 3.1-1 Specific heat capacity database for Zircaloy-2, Zircaloy-4, ZIRLO, and M5..3.2

Table 3.1-2 Specific heat capacity database for ZrNb-1 alloys..........................................3.2

Table 3.8-1 Parameters for FRAPCON-3.4 creep equation for SRA and RXA cladding. 3.31

Table 4.1-1 Constants used in gas thermal conductivity correlations in MATPRO............4.1

Table 4.1-2 Constants used in gas thermal conductivity correlations in FRAP. ..................4.2

Table 4.1-3 Standard deviation of gas thermal conductivity correlations............................4.8 


\section{Executive Summary}

The U.S. Nuclear Regulatory Commission uses the computer codes FRAPCON-3 and FRAPTRAN to model steady-state and transient fuel behavior, respectively, in regulatory analysis. In this document, material property correlations for oxide fuels and cladding materials are presented and discussed. Comparisons are made between the material property correlations used in the most recent versions of the codes, FRAPCON-3.4 and FRAPTRAN 1.4. Comparisons are also made with MATPRO, which is a compilation of material property correlations with an extensive history of use with various fuel performance and severe accident codes. In addition to model-to-model comparisons, model-to-data comparisons and source codes for the FRAPCON-3.4 and FRAPTRAN 1.4 correlations are also provided.

This is one of three documents that describe the FRAPCON-3.4 and FRAPTRAN 1.4 fuel performance codes. The FRAPCON-3.4 code structure and behavioral models are described in the FRAPCON-3.4 code description document (Geelhood et al., 2010a). The FRAPTRAN 1.4 code structure and behavioral models are described in the FRAPTRAN 1.4 code description document (Geelhood et al., 2010b). Additional descriptions of mechanical models and model-todata comparisons for mechanical property correlations are provided in the mechanical properties report (Geelhood, 2008). 


\section{Abbreviations}

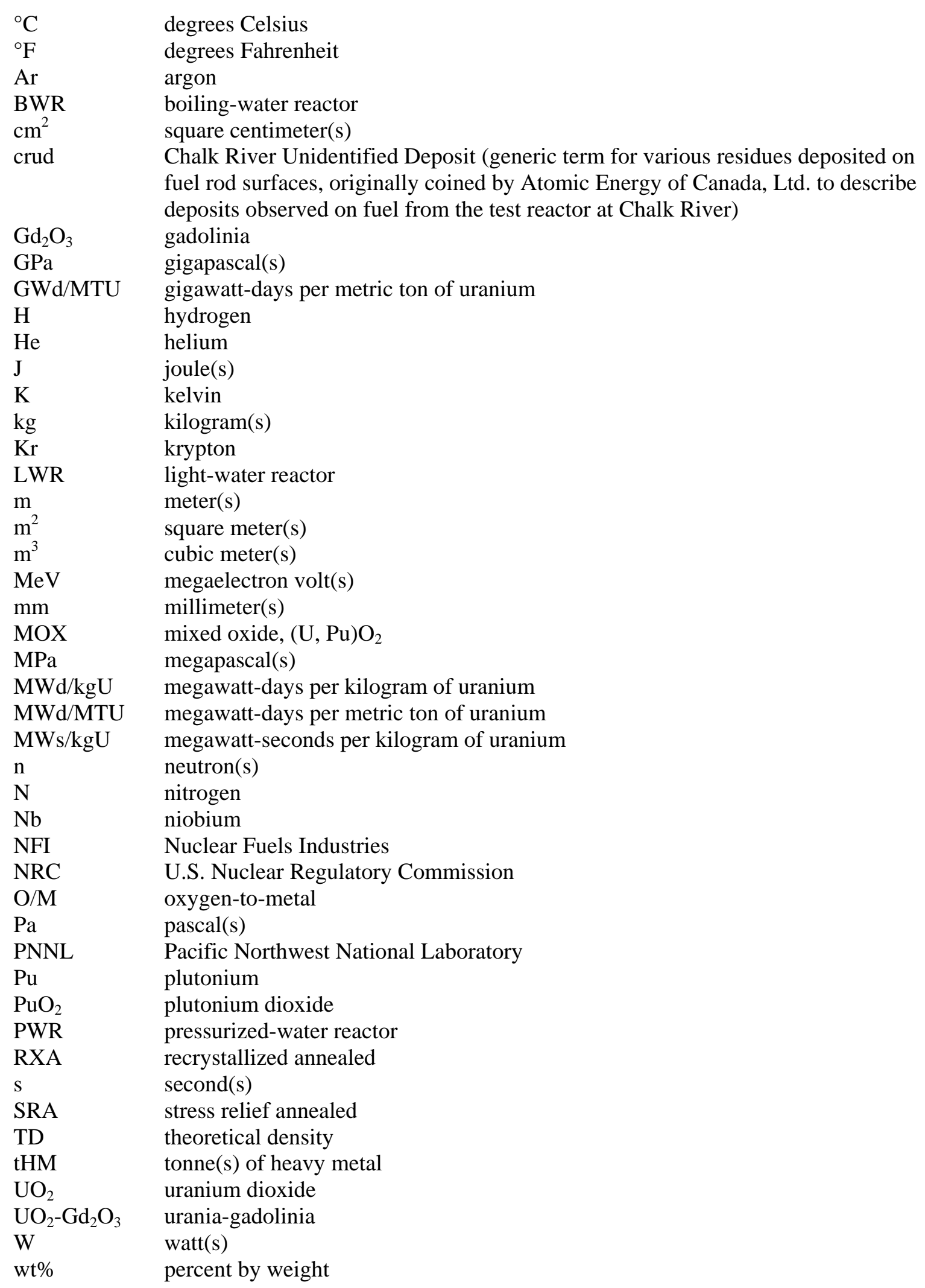


$\mathrm{Xe}$

ZrNb-1

$\mathrm{ZrO}_{2}$

$\mu \mathrm{m}$

xenon

Russian zirconium alloy with $1 \mathrm{wt} \%$ of niobium zirconium dioxide

micrometer(s) 


\subsection{Introduction}

The U.S. Nuclear Regulatory Commission (NRC) uses the computer codes FRAPCON-3 and FRAPTRAN to model steady-state and transient fuel behavior, respectively, in regulatory analysis. To effectively model fuel behavior, material property correlations must be used for a wide range of operating conditions (e.g., temperature and burnup). In this sense, a "material property" is a physical characteristic of the material whose quantitative value is necessary in the analysis process. Further, the property may be used to compare the benefits of one material with those of another. Generally speaking, the material properties of interest in regulatory analysis of nuclear fuel behavior are mechanical or thermodynamic.

The issue of what is and is not a "material property" will never be universally resolved. In this report, properties such as thermal conductivity are included. Other characteristics of the material (e.g., fission gas release) are considered "models" rather than properties and are discussed elsewhere. The material property correlations for the FRAPCON-3 and FRAPTRAN computer codes were partially documented in NUREG/CR-6534 and NUREG/CR-6739, respectively (Lanning et al., 2005; Cunningham et al., 2001) as well as in NUREG/CR-6150 (Siefken et al., 2001). Some of these have been modified or updated since the original code documentation was published.

The primary purpose of this report is to consolidate the current material property correlations used in FRAPCON-3 and FRAPTRAN into one document. Material property correlations for oxide fuels, including uranium dioxide $\left(\mathrm{UO}_{2}\right)$ and mixed oxide (MOX) fuels, are described in Section 2. Throughout this document, the term MOX is used to describe fuels that are blends of uranium and plutonium oxides, $(\mathrm{U}, \mathrm{Pu}) \mathrm{O}_{2}$. The properties for $\mathrm{UO}_{2}$ with other additives (e.g., gadolinia) are also discussed. Material property correlations for cladding materials and gases are described in Sections 3 and 4, respectively.

In addition to describing the material property correlations used in the subroutines of FRAPCON-3 and FRAPTRAN, this report provides a variety of comparisons between material property correlations and data. Although they are frequently identical, comparisons are made between the material property correlations used in the FRAPCON-3 and FRAPTRAN codes. Comparisons are also made between the material property correlations used in MATPRO, a compilation of fuel and cladding material property correlations with an extensive history of use with various fuel performance and severe accident codes. For several reasons, consistency among the material property correlations in FRAPCON-3, FRAPTRAN, and MATPRO has never been complete. However, the current versions of FRAPCON-3 and FRAPTRAN use a relatively consistent set of correlations for the properties that are used by both codes. The material property correlations in the most recent version of MATPRO are documented in Volume 4 of NUREG/CR-6150 (Siefken et al., 2001). In addition to comparison of the various correlations, correlation-to-data comparisons are made with FRAPCON-3, FRAPTRAN, and MATPRO properties.

All comparisons in this report are based on the material property correlations used in the most recent version of the FRAPCON-3 and FRAPTRAN codes, FRAPCON-3.4 and FRAPTRAN 1.4. The source code for each material property correlation discussed is provided for FRAPCON-3.4 and FRAPTRAN 1.4 (see Appendix A) as well as a range of applicability and an estimate of uncertainty, where possible. 


\subsection{Relation to Other Reports}

The full documentation of the steady-state and transient fuel performance codes is described in three documents. The basic fuel, cladding, and gas material properties used in FRAPCON-3.4 and FRAPTRAN 1.4 are described in the material properties handbook (this report). The FRAPCON-3.4 code structure and behavioral models are described in the FRAPCON-3.4 code description document (Geelhood et al., 2010a). The FRAPTRAN 1.4 code structure and behavioral models are described in the FRAPTRAN 1.4 code description document (Geelhood et al., 2010b). Additional descriptions of mechanical models and model-to-data comparisons for mechanical property correlations are provided in the mechanical properties report (Geelhood, 2008).

Table 1.1 shows where each specific material property and model used in the NRC fuel performance codes are documented.

Table 1.1 Roadmap to documentation of models and properties in NRC fuel performance codes, FRAPCON-3.4 and FRAPTRAN 1.4.

\begin{tabular}{|l|l|l|}
\hline Model/Property & FRAPCON-3.4 & FRAPTRAN 1.4 \\
\hline Fuel thermal conductivity & Material properties handbook & Material properties handbook \\
\hline Fuel thermal expansion & Material properties handbook & Material properties handbook \\
\hline Fuel melting temperature & Material properties handbook & Material properties handbook \\
\hline Fuel specific heat & Material properties handbook & Material properties handbook \\
\hline Fuel enthalpy & Material properties handbook & Material properties handbook \\
\hline Fuel emissivity & Material properties handbook & Material properties handbook \\
\hline Fuel densification & Material properties handbook & NA \\
\hline Fuel swelling & Material properties handbook & NA \\
\hline Fission gas release & FRAPCON-3 code description & FRAPTRAN code description \\
\hline Fuel relocation & FRAPCON-3 code description & FRAPTRAN code description \\
\hline Fuel grain growth & & \\
\hline High burnup rim model & FRAPCON-3 code description & NA \\
\hline Nitrogen release & FRAPCON-3 code description & NA \\
\hline Helium release & FRAPCON-3 code description & NA \\
\hline Radial power profile & FRAPCON-3 code description & NA (input parameter) \\
\hline Stored energy & FRAPCON-3 code description & FRAPTRAN code description \\
\hline Decay heat model & NA & FRAPTRAN code description \\
\hline $\begin{array}{l}\text { Fuel and cladding temperature } \\
\text { solution }\end{array}$ & FRAPCON-3 code description & FRAPTRAN code description \\
\hline Cladding thermal conductivity & Material properties handbook & Material properties handbook \\
\hline Cladding thermal expansion & Material properties handbook & Material properties handbook \\
\hline Cladding elastic modulus & Material properties handbook & Material properties handbook \\
\hline Cladding creep model & Material properties handbook & NA \\
\hline Cladding specific heat & Material properties handbook & Material properties handbook \\
\hline
\end{tabular}




\begin{tabular}{|c|c|c|}
\hline Model/Property & FRAPCON-3.4 & FRAPTRAN 1.4 \\
\hline Cladding emissivity & Material properties handbook & Material properties handbook \\
\hline Cladding axial growth & Material properties handbook & NA \\
\hline Cladding Meyer hardness & Material properties handbook & Material properties handbook \\
\hline Cladding annealing & FRAPCON-3 code description & FRAPTRAN code description \\
\hline $\begin{array}{l}\text { Cladding yield stress, ultimate } \\
\text { stress, and plastic deformation }\end{array}$ & FRAPCON-3 code description & FRAPTRAN code description \\
\hline Cladding failure criteria & NA & FRAPTRAN code description \\
\hline Cladding waterside corrosion & FRAPCON-3 code description & NA (input parameter) \\
\hline Cladding hydrogen pickup & FRAPCON-3 code description & NA (input parameter) \\
\hline $\begin{array}{l}\text { Cladding high temperature } \\
\text { oxidation }\end{array}$ & NA & FRAPTRAN code description \\
\hline Cladding ballooning model & NA & FRAPTRAN code description \\
\hline Cladding mechanical deformation & FRAPCON-3 code description & FRAPTRAN code description \\
\hline Oxide thermal conductivity & Material properties handbook & Material properties handbook \\
\hline Crud thermal conductivity & FRAPCON-3 code description & NA \\
\hline Gas conductivity & Material properties handbook & Material properties handbook \\
\hline Gap conductance & FRAPCON-3 code description & FRAPTRAN code description \\
\hline Plenum gas temperature & FRAPCON-3 code description & FRAPTRAN code description \\
\hline Rod internal pressure & FRAPCON-3 code description & FRAPTRAN code description \\
\hline $\begin{array}{l}\text { Coolant temperature and heat } \\
\text { transfer coefficients }\end{array}$ & FRAPCON-3 code description & FRAPTRAN code description \\
\hline \multicolumn{3}{|c|}{ Optional models and properties not developed at PNNL } \\
\hline VVER fuel and cladding models & NA & $\begin{array}{l}\text { NUREG/IA-0164 } \\
\text { (Shestopalov et al., 1999) }\end{array}$ \\
\hline Cladding FEA model & $\begin{array}{l}\text { VTT-R-11337-06 } \\
\text { (Knuttilla, 2006) }\end{array}$ & $\begin{array}{l}\text { VTT-R-11337-06 } \\
\text { (Knuttilla, 2006) }\end{array}$ \\
\hline
\end{tabular}

NA = not applicable

VVER = water-cooled, water-moderated energy reactor

FEA = finite element analysis

\subsection{References}

Lanning, D.D., C.E. Beyer, and K.J. Geelhood. 2005. FRAPCON-3 Updates, Including MixedOxide Fuel Properties, NUREG/CR-6534, Vol. 4, PNNL-11513, Pacific Northwest National Laboratory, Richland, WA.

Cunningham, M.E., C.E. Beyer, F.E. Panisko, P.G. Medvedev, G.A. Berna, and H.H. Scott. 2001. FRAPTRAN: Integral Assessment, NUREG/CR-6739, Vol. 2, PNNL-13576, Pacific Northwest National Laboratory, Richland, WA. 
Siefken, L.J., E.W. Coryell, E.A. Harvego, and J.K. Hohorst. 2001. SCDAP/RELAP5/MOD 3.3 Code Manual MATPRO-A Library of Materials Properties for Light-Water-Reactor-Accident Analysis, NUREG/CR-6150, Vol. 4, Rev. 2, INEL-96/0422, Idaho National Engineering and Environmental Laboratory, Idaho Falls, ID.

Geelhood, K.J., W.G. Luscher, and C.E. Beyer. 2010a. FRAPCON-3.4: A Computer Code for the Calculation of Steady-State, Thermal-Mechanical Behavior of Oxide Fuel Rods for High Burnup, NUREG/CR-7022, Vol. 1, PNNL-19418, Vol. 1, Pacific Northwest National Laboratory, Richland, WA.

Geelhood, K.J., W.G. Luscher, C.E. Beyer, and J.M. Cuta. 2010b. FRAPTRAN 1.4: A Computer Code for the Transient Analysis of Oxide Fuel Rods, NUREG/CR-7023, Vol. 1, PNNL-19400, Vol. 1, Pacific Northwest National Laboratory, Richland, WA.

Geelhood, K.J., W.G. Luscher, and C.E. Beyer. 2008. PNNL Stress/Strain Correlation for Zircaloy, PNNL-17700, Pacific Northwest National Laboratory, Richland, WA.

Shestopalov, A., K. Lioutov, L. Yegorova, G. Abyshov, and K. Mikitiouk. 1999. Modification of USNRC's FRAP-T6 Fuel Rod Transient Code for High Burnup VVER Fuel, NUREG/IA-0164, U.S. Nuclear Regulatory Commission, Washington, D.C.

Knuutila, A. 2006. Improvements on FRAPCON-3/FRAPTRAN Mechanical Modeling, VTT-R11337-06, VTT, Finland. 


\subsection{Oxide Fuel Properties}

Material property correlations for $\mathrm{UO}_{2}$ and $\mathrm{MOX}$ fuel pellets are described in the following subsections. These correlations include the melting temperature, specific heat capacity and enthalpy, thermal conductivity, emissivity, thermal expansion, densification, and swelling of the fuel pellets. When indicated, some correlations account for gadolinia $\left(\mathrm{Gd}_{2} \mathrm{O}_{3}\right)$ additions in $\mathrm{UO}_{2}$ fuel pellets. The subroutine containing the material correlation in the FRAPCON-3/FRAPTRAN codes is given in parentheses in the heading of each subsection.

\subsection{Fuel Melting Temperature (PHYPRP)}

The subroutine PHYPRP is used to calculate the temperature of the first appearance of liquid phase (solidus) and the temperature of the melting point of the last solid phase (liquidus) of $\mathrm{UO}_{2}$ and MOX. These temperatures are calculated as a function of burnup and plutonia content. The same subroutine is used in both FRAPCON-3.4 and FRAPTRAN 1.4 and exhibits smaller burnup dependence than the corresponding MATPRO correlation, FHYPRP.

\subsubsection{Model Development and Comparisons}

The equations used to calculate the $\mathrm{UO}_{2}$ and $\mathrm{MOX}$ melting points were derived by using 3113.15K as the melting temperature of urania, which was determined experimentally by Brassfield (1968), and a least squares fit to parabolic equations for the solidus and liquidus boundaries from the Lyon and Baily (1967) phase diagram for the stoichiometric (U, $\mathrm{Pu}) \mathrm{O}_{2}$ mixed oxide. These correlations are presented in Equations 2.1-1 through 2.1-5.

$$
\begin{aligned}
& \text { sldus }(c)=2840-5.41395 c+7.468390 \times 10^{-3} c^{2} \\
& \text { liqdus }(c)=2840-3.21860 c+1.448518 \times 10^{-2} c^{2}
\end{aligned}
$$

For $\mathrm{c}=0$

$$
\text { ftmelt }=3113.15-5 \mathrm{FBu} / 10000
$$

For c $>0$

$$
\begin{gathered}
\text { ftmelt }=\text { sldus (c) }+273.15-5 \text { FBu } / 10000 \\
\text { fdelta = liqdus (c) }- \text { sldus (c) }-5 \text { FBu } / 10000
\end{gathered}
$$

where

$\begin{array}{lll}\mathrm{C} & = & \text { Pu content (wt\%) } \\ \text { sldus (c) } & = & \text { solidus temperature as a function of Pu content }\left({ }^{\circ} \mathrm{C}\right) \\ \text { liqdus (c) } & = & \text { liquidus temperature as a function of Pu content }\left({ }^{\circ} \mathrm{C}\right) \\ \text { ftmelt } & = & \text { fuel melting temperature }(\mathrm{K}) \\ \mathrm{FBu} & = & \text { burnup (MWd/MTU) } \\ \text { fdelta } & = & \text { temperature range between solidus and liquidus (K) }\end{array}$


The correlations defined above for the subroutine PHYPRP are almost identical to those used in the subroutine FHYPRP documented in MATPRO. However, the burnup dependence of the PHYPRP subroutine differs from the FHYPRP subroutine. A burnup dependence of $3.2 \mathrm{~K} / \mathrm{GWd} / \mathrm{MTU}$ is used in FHYPRP, while a burnup dependence of $0.5 \mathrm{~K} / \mathrm{GWd} / \mathrm{MTU}$ is used in PHYPRP. This change was suggested by Popov et al. (2000) and is based on data from Adamson et al. (1985) and Komatsu et al. (1988).

The burnup-dependent term in Equations 2.1-3 through 2.1-5 reflects this change in burnup dependence. Also, the term $3.21860 \mathrm{c}$ in Equation 2.1-2 is slightly different from the term used in FHYPRP. The coefficient was changed from 3.21660 in FHYPRP to 3.21860 in PHYPRP. However, this change does not appreciably affect the liquidus temperature.

Comparisons between solidus and liquidus temperature calculated by PHYPRP and FHYPRP are presented in Figures 2.1-1 and 2.1-2. Both subroutines predict solidus and liquidus temperatures that decrease with increasing burnup and plutonium concentration. However, these figures reveal a smaller decline in solidus and liquidus temperatures for the FRAPCON-3 and FRAPTRAN subroutine (PHYPRP) than the MATPRO subroutine (FHYPRP). This is a result of reducing the burnup dependence from 3.2 to $0.5 \mathrm{~K} / \mathrm{GWd}$ /MTU between the FHYPRP and PHYPRP subroutines, respectively.

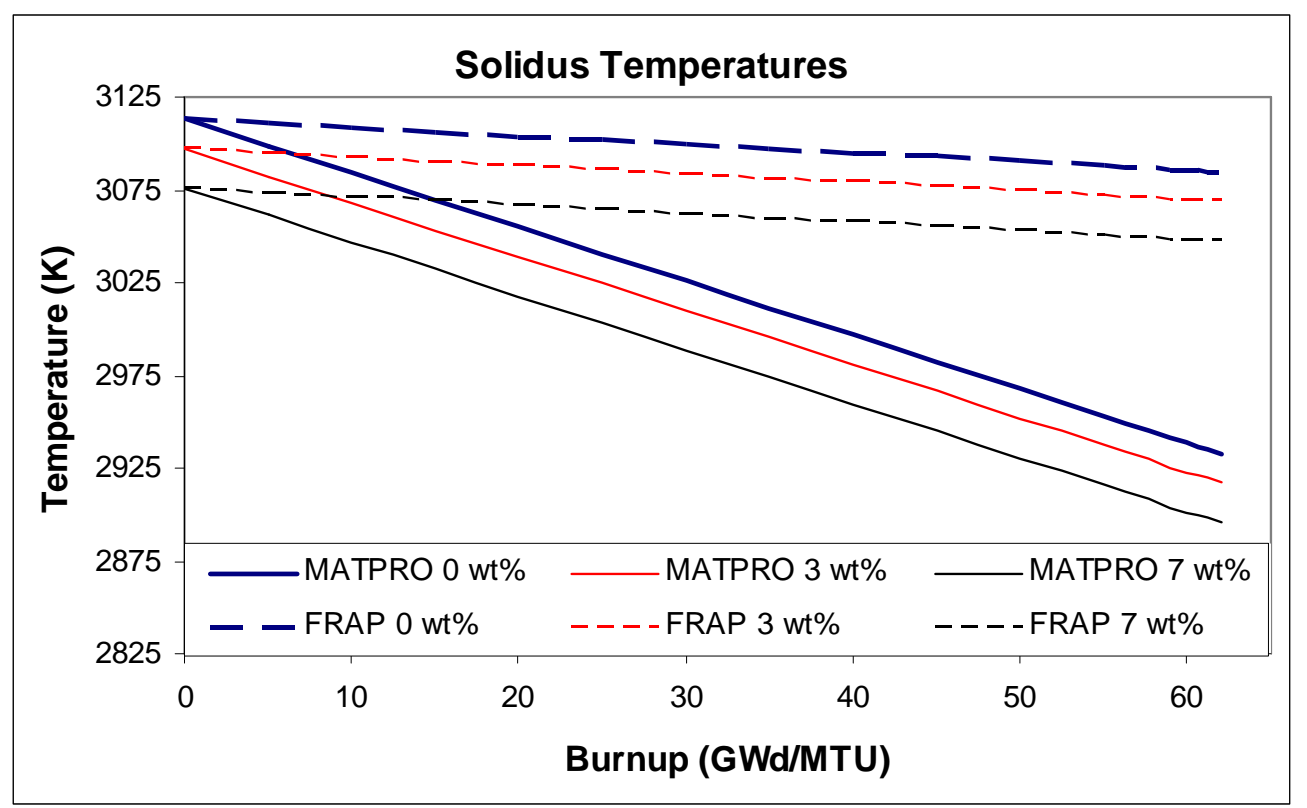

Figure 2.1-1 A comparison between solidus temperatures calculated by MATPRO using the FHYPRP subroutine and FRAPCON-3/FRAPTRAN (FRAP) using the PHYPRP subroutine. Results for burnup varying from 0 to $62 \mathrm{GWd} / \mathrm{MTU}$ and Pu contents of 0,3 , and $7 \mathrm{wt} \%$ are presented. 


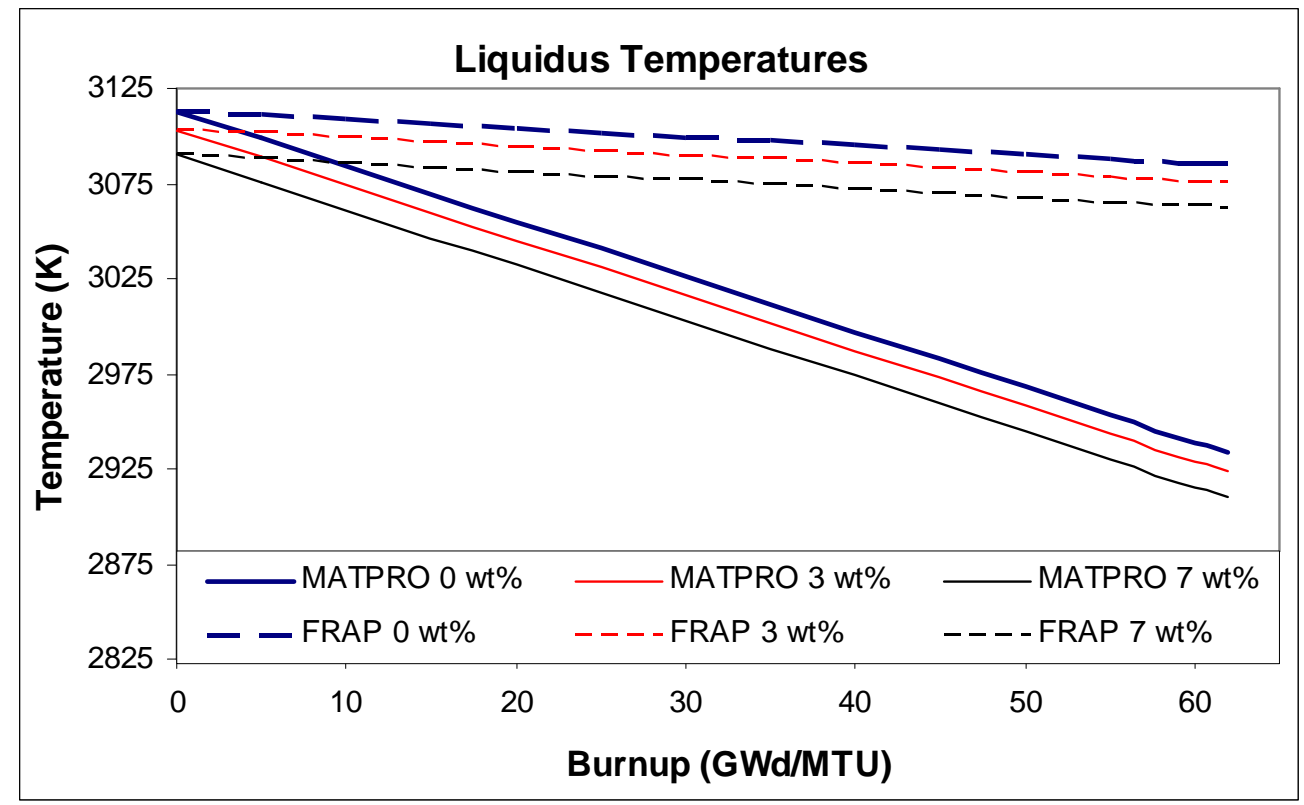

Figure 2.1-2 A comparison between liquidus temperatures calculated by MATPRO using the FHYPRP subroutine and FRAPCON-3/FRAPTRAN (FRAP) using the PHYPRP subroutine. Results for burnup varying from 0 to $62 \mathrm{GWd} / \mathrm{MTU}$ and $\mathrm{Pu}$ contents of 0,3 , and $7 \mathrm{wt} \%$ are presented.

\subsubsection{Applicability and Uncertainty}

The correlation used in PHYPRP is valid through the melting temperature of $\mathrm{UO}_{2}$ and $\mathrm{MOX}$ for burnups up to $62 \mathrm{GWd} / \mathrm{MTU}$. Currently, there are no models for urania-gadolinia $\left(\mathrm{UO}_{2}-\mathrm{Gd}_{2} \mathrm{O}_{3}\right)$ fuel melting. However, there are plans to develop a correlation in a future revision of the code. Due to the experimental data and data interpretations on which these temperature correlations are based, it is difficult to express a quantitative measure of uncertainty. A conservative estimate of uncertainty based on engineering judgment is $\pm 50 \mathrm{~K}$.

\subsubsection{References}

Brassfield, H.C., et al. 1968. Recommended Property and Reactor Kinetics Data for Use in Evaluating a Light-Water-Coolant Reactor Loss-of-Coolant Incident Involving Zircaloy-4 or 304SS-Clad $\mathrm{UO}_{2}$, GEMP-482, General Electric Company, Cincinnati, OH.

Lyon, W.F., and W.E. Baily. 1967. "The Solid Liquid Phase Diagram for the $\mathrm{UO}_{2}-\mathrm{PuO}_{2}$ System,” Journal of Nuclear Materials, 22:332.

Popov, S.G., J.J. Carbajo, V.K. Ivanov, and G.L. Yoder. 2000. Thermophysical Properties of MOX and $\mathrm{UO}_{2}$ Fuel Including the Effects for Irradiation, ORNL/TM 2000/351, Oak Ridge National Laboratory, Oak Ridge, TN.

Adamson, M.G., E.A. Aitken, and R.W. Caputi. 1985. "Experimental and thermodynamic evaluation of the melting behavior of irradiated oxide fuels." Journal of Nuclear Materials, 130:349-365. 
Komatsu, J., T. Tachibana, and K. Konashi. 1988. "The Melting Temperature of Irradiated Oxide Fuel,” Journal of Nuclear Materials, 154:38-44.

\subsection{Fuel Specific Heat Capacity (FCP) and Fuel Enthalpy (FENTHL)}

The subroutines FCP and FENTHL are used to calculate the specific heat capacity and enthalpy of the fuel pellet, respectively. The specific heat capacity and enthalpy of nuclear fuel are modeled empirically as functions of four parameters: temperature, composition, molten fraction, and oxygen-to-metal $(\mathrm{O} / \mathrm{M})$ ratio. The same subroutine is used in FRAPCON-3.4, FRAPTRAN 1.4, and MATPRO.

\subsubsection{Model Development and Comparisons}

Equations for the specific heat capacity and enthalpy of solid $\mathrm{UO}_{2}$ and plutonium dioxide $\left(\mathrm{PuO}_{2}\right)$ are assumed to have the same form, but with different constants. The basic relationships are presented in Equations 2.2-1 and 2.2-2, respectively.

$$
\begin{aligned}
& \mathrm{FCP}=\frac{\mathrm{K}_{1} \theta^{2} \exp (\theta / \mathrm{T})}{\mathrm{T}^{2}[\exp (\theta / \mathrm{T})-1]^{2}}+\mathrm{K}_{2} \mathrm{~T}+\frac{\mathrm{YK}_{3} \mathrm{E}_{\mathrm{D}}}{2 \mathrm{RT}} \exp \left(-\mathrm{E}_{\mathrm{D}} / \mathrm{RT}\right) \\
& \text { FENTHL }=\frac{\mathrm{K}_{1} \theta}{\exp (\theta / \mathrm{T})-1}+\frac{\mathrm{K}_{2} \mathrm{~T}^{2}}{2}+\frac{\mathrm{Y}}{2}\left[\mathrm{~K}_{3} \exp \left(-\mathrm{E}_{\mathrm{D}} / \mathrm{RT}\right)\right]
\end{aligned}
$$

where

$\begin{array}{lll}\text { FCP } & = & \text { specific heat capacity }\left(\mathrm{J} / \mathrm{kg}^{*} \mathrm{~K}\right) \\ \mathrm{FENTHL} & = & \text { fuel enthalpy }(\mathrm{J} / \mathrm{kg}) \\ \mathrm{T} & = & \text { temperature }(\mathrm{K}) \\ \mathrm{Y} & = & \text { oxygen-to-metal ratio } \\ \mathrm{R} & = & \text { universal gas constant } \quad=\quad 8.3143(\mathrm{~J} / \mathrm{mol} * \mathrm{~K}) \\ \theta & = & \text { the Einstein temperature }(\mathrm{K}) \\ \mathrm{E}_{\mathrm{D}} & = & \text { activation energy for Frenkel defects }(\mathrm{J} / \mathrm{mol})\end{array}$

and the constants are given in Table 2.2-1. 
Table 2.2-1 Constants used in $\mathrm{UO}_{2}, \mathrm{Gd}_{2} \mathrm{O}_{3}$, and $\mathrm{PuO}_{2}$ heat capacity and enthalpy correlations.

\begin{tabular}{|c|c|c|c|c|}
\hline Constant & $\mathbf{U O}_{\mathbf{2}}$ & $\mathbf{P u O}_{\mathbf{2}}$ & $\mathbf{G d O}_{2}{ }^{*}$ & Units \\
\hline $\mathrm{K}_{1}$ & 296.7 & 347.4 & 315.86 & $\mathrm{~J} / \mathrm{kg}{ }^{*} \mathrm{~K}$ \\
\hline $\mathrm{K}_{2}$ & $2.43 \times 10^{-2}$ & $3.95 \times 10^{-4}$ & $4.044 \times 10^{-2}$ & $\mathrm{~J} / \mathrm{kg}^{*} \mathrm{~K}^{2}$ \\
\hline $\mathrm{K}_{3}$ & $8.745 \times 10^{7}$ & $3.860 \times 10^{7}$ & 0.0 & $\mathrm{~J} / \mathrm{kg}$ \\
\hline$\theta$ & 535.285 & 571.000 & 348.0 & $\mathrm{~K}$ \\
\hline $\mathrm{E}_{\mathrm{D}}$ & $1.577 \times 10^{5}$ & $1.967 \times 10^{5}$ & 0.0 & $\mathrm{~J} / \mathrm{mol}$ \\
\hline
\end{tabular}

* Gadolinia additions are only accounted for in FRAPCON-3.4.

The constants in Table 2.2-1 were determined by Kerrisk and Clifton (1972) for $\mathrm{UO}_{2}$ and Kruger and Savage (1968) for $\mathrm{PuO}_{2}$. The specific heat capacity of $\mathrm{UO}_{2}$ in the liquid state (see Equation 2.2-3) was determined by Leibotwitz (1971) and assumed to be valid for $\mathrm{PuO}_{2}$ in the liquid state.

$$
\mathrm{FCP}_{\text {Liquid }} \quad=503 \mathrm{~J} / \mathrm{kg}^{*} \mathrm{~K}
$$

Inspection of Equations 2.2-1 and 2.2-2 reveals that fuel enthalpy, or stored energy, is calculated by integrating the fuel specific heat capacity correlation from 0 to $\mathrm{T}$ in kelvin. Stored energy is important in reactor transient analysis because the severity of the transient is significantly affected by the initial stored energy of the fuel. Since $\mathrm{UO}_{2}$ and $\mathrm{PuO}_{2}$ are the principal oxides in light-water reactor (LWR) fuels, they are the constituents considered.

The correlations for specific heat and enthalpy in both $\mathrm{UO}_{2}$ and MOX fuel pellets are identical in FRAPCON-3.4, FRAPTRAN 1.4, and MATPRO. However, FRAPCON-3.4 can accommodate gadolinia additions whereas FRAPTRAN cannot. For a mixture of $\mathrm{UO}_{2}, \mathrm{Gd}_{2} \mathrm{O}_{3}$, and $\mathrm{PuO}_{2}$, the specific heat capacity of the solid is determined by combining the contribution from each constituent in proportion to its weight fraction. When the material is partially molten, the heat capacity is determined similarly with a weighted sum of the solid and molten fractions.

Since the specific heat correlation is only valid above a fuel temperature of about $300 \mathrm{~K}$, the fuel enthalpy correlation is not valid below a temperature of about $300 \mathrm{~K}$. In addition, it is necessary to calculate fuel enthalpy with respect to a reference temperature $\geq 300 \mathrm{~K}$. Thus, the fuel enthalpy at any desired temperature, $\mathrm{T}$, is calculated by evaluating Equation 2.2-2 at $\mathrm{T}$ and a reference temperature, $\mathrm{T}_{\text {ref }}$, of $300 \mathrm{~K}$ and taking the difference [FENTHL(T) - FENTHL( $\left.\mathrm{T}_{\text {ref }}\right)$ ]. For temperatures greater than $2 \mathrm{~K}$ below melting, the molten fraction and heat of fusion are used to interpolate between the enthalpy of unmelted fuel and just-melted fuel at the melting temperature.

Specific heat correlations are compared with independent experimental data in Figure 2.2-1 and Figure 2.2-2 for $\mathrm{UO}_{2}$ and $\mathrm{PuO}_{2}$, respectively. Comparison between the $\mathrm{UO}_{2}$ model and experimental data from Hein (1968), Leibowitz (1969), and Gronvold (1970) in Figure 2.2-1 reveals agreement between predicted and measured values up to high temperatures (a few hundred kelvin below melting). At this point the data begin to fall lower than the model. This is attributed to partial melting due to a non-uniform temperature distribution within the sample. With the exception of the Affortit (1970) data, relatively good agreement is observed in Figure 2.2-2, which compares the values measured by Gibby (1974), Leibowitz (1972), and Affortit with predicted heat capacities for a MOX fuel, $\left(\mathrm{U}_{0.8}, \mathrm{Pu}_{0.2}\right) \mathrm{O}_{2}$. Since the Affortit results are known to be generally low in comparison to results from other investigators, the correlation is considered to be in good agreement with the experimental data. 


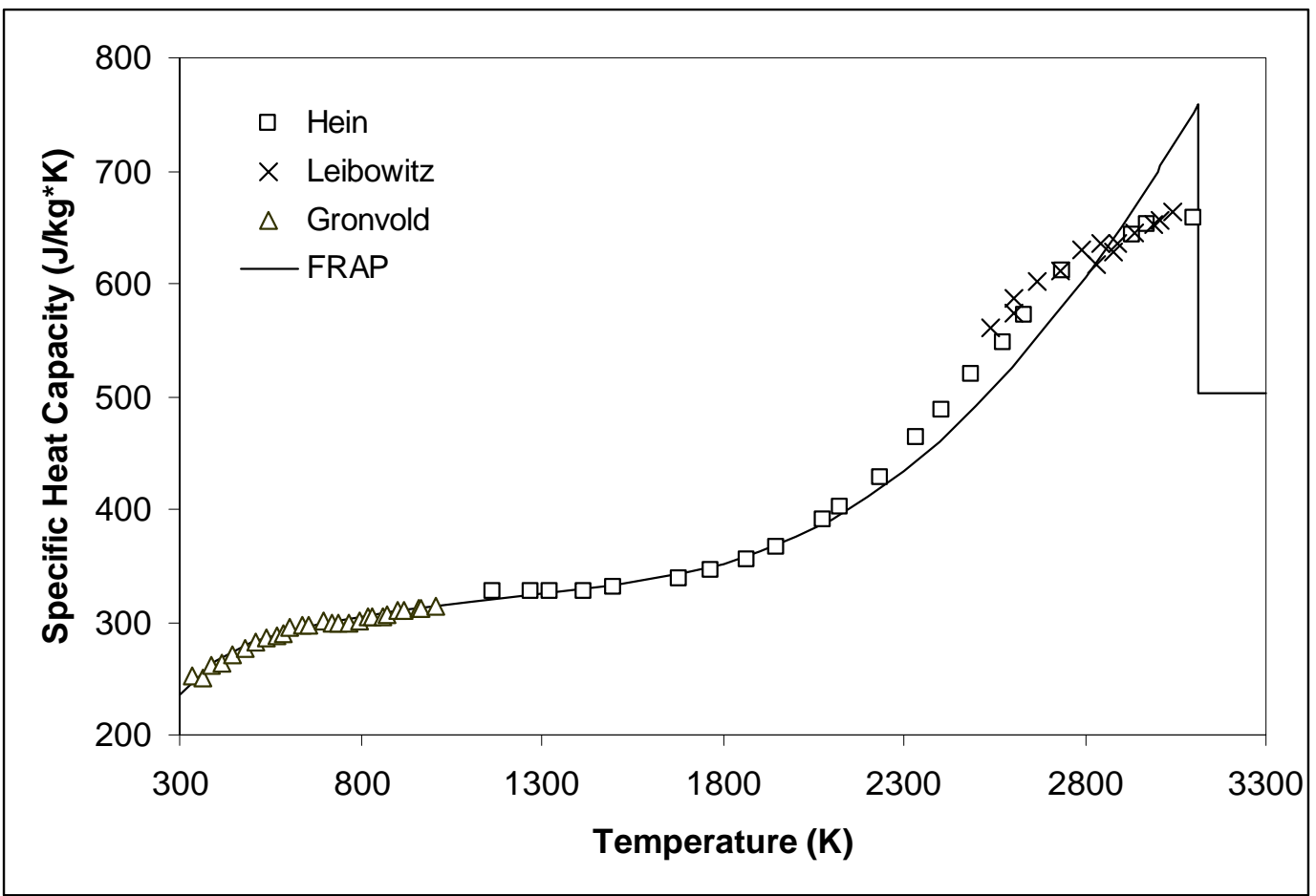

Figure 2.2-1 Specific heat capacity of $\mathrm{UO}_{2}$ from three experimenters compared with the FRAPCON-3/FRAPTRAN/MATPRO (FRAP) correlation (solid line) for $\mathrm{UO}_{2}$. 


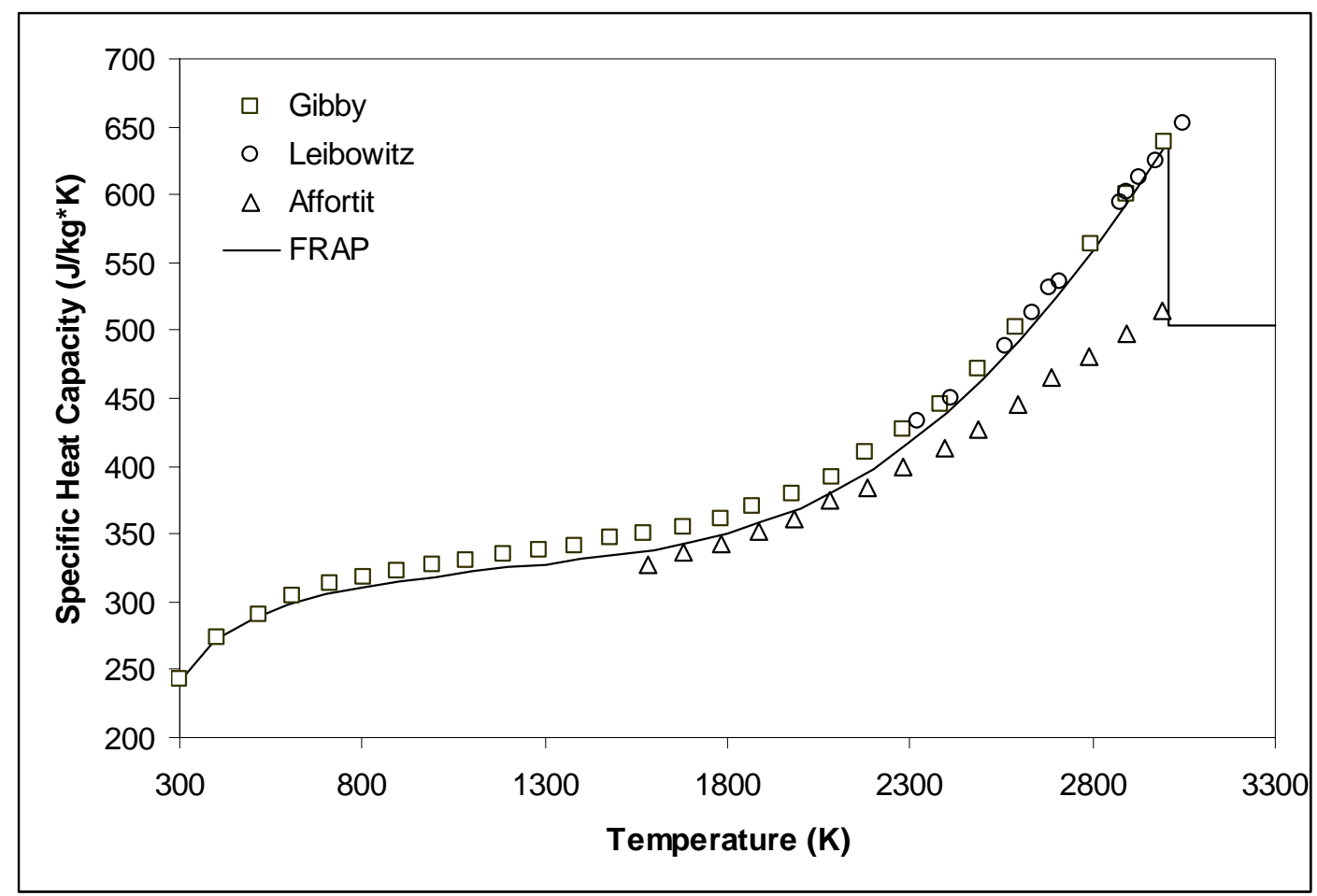

Figure 2.2-2 Specific heat capacity of $\left(\mathrm{U}_{0.8} \mathrm{Pu}_{0.2}\right) \mathrm{O}_{2}$ from three experimenters compared with the FRAPCON-3/FRAPTRAN/MATPRO (FRAP) correlations (solid line) for MOX.

\subsubsection{Applicability and Uncertainty}

The correlations for fuel specific heat and enthalpy are valid for temperatures from $300 \mathrm{~K}$ to more than $4000 \mathrm{~K}$. The standard error of the $\mathrm{UO}_{2}$ specific heat capacity correlation is $\pm 3 \mathrm{~J} / \mathrm{kg}^{*} \mathrm{~K}$. However, this error increases at temperatures above 2300K, as seen in Figure 2.2-1, and may lead to underpredictions and overpredictions of 59 and $66 \mathrm{~J} / \mathrm{kg}^{*} \mathrm{~K}$, respectively. As for the mixedoxide-specific heat capacity correlation, it is 6 to $10 \mathrm{~J} / \mathrm{kg}^{*} \mathrm{~K}$, depending on the fraction of $\mathrm{PuO}_{2}$. For nonstoichiometric fuels, these uncertainties are approximately double.

\subsubsection{References}

Kerrisk, J.F., and D.G. Clifton. 1972. "Smoothed Values for the Enthalpy and Heat Capacity of $\mathrm{UO}_{2}$," Nuclear Technology, 16:531-535.

Kruger, O.L., and H. Savage. 1968. "Heat Capacity and Thermodynamic Properties of Plutonium Dioxide,” Journal of Chemical Physics, 45:4540-4544.

Leibowitz, L., et al. 1971. "Enthalpy of Liquid Uranium Dioxide to 3500 K,” Journal of Nuclear Materials, 39:115-116.

Hein, R.A., L.H. Sjodahl, and R. Swarc. 1968. "Heat Content of Uranium Dioxide from 1,200 to 3,100 K,” Journal of Nuclear Materials, 25:99-102. 
Leibowitz, L., L.W. Mschler, and M.G. Chasanov. 1969. "Enthalpy of Solid Uranium Dioxide from 2,500 K to its Melting Point,” Journal of Nuclear Materials, 29:356-358.

Gronvold, F., et al. 1970. "Thermodynamics of the $\mathrm{UO}_{2+x}$ Phase I. Heat Capacities of $\mathrm{UO}_{2.017}$ and $\mathrm{UO}_{2.254}$ from 300 to $1,000 \mathrm{~K}$ and Electronic Contributions,” Journal of Chemical Thermodynamics, 2:665-679.

Affortit, C., and J. Marcon. 1970. "Chaleur Specifique a Haute Temperature des Oxydes D'Uranium et de Plutonium,” Revue Internationale des Hautes Temperatures et des Refractaires, 7:236-241.

Gibby, R.L., et al. 1974. "Analytical Expressions for Enthalpy and Heat Capacity for UraniumPlutonium Oxide,” Journal of Nuclear Materials, 50:155-161.

Leibowitz, L., D.F. Fischer, and M.G. Chasanov. 1972. "Enthalpy of Uranium Plutonium Oxides (U0.8,Pu0.2) $\mathrm{O}_{1.07}$ from 2,350 to 3,000 K,” Journal of Nuclear Materials, 42:113-116.

\subsection{Fuel Thermal Conductivity (FTHCON)}

The subroutine FTHCON is used to calculate the thermal conductivity of the fuel pellet. Since accurate predictions of fuel rod behavior are strongly dependent on temperature, the calculation of fuel thermal conductivity is critical. The FTHCON subroutine is used in FRAPCON-3.4, FRAPTRAN 1.4, and MATPRO. However, the calculation of fuel thermal conductivity has evolved from the model originally proposed in MATPRO to the modified Nuclear Fuels Industries (NFI) and Duriez/modified NFI models used in the FRAPCON-3.4 and FRAPTRAN 1.4 codes for $\mathrm{UO}_{2}, \mathrm{UO}_{2}-\mathrm{Gd}_{2} \mathrm{O}_{3}$, and $\mathrm{MOX}$, respectively. An additional fuel thermal conductivity model for MOX developed at Halden is included as an option in FRAPCON-3.4. Each of the models is discussed in detail in the following sections along with the evolution of the MATPRO model and the applicability and uncertainty of the models.

Following the initial description of the MATPRO fuel thermal conductivity model, an overview of subsequent thermal conductivity models is given. This historical summary of how thermal conductivity modeling has evolved provides insight to model development and previous motivations for change. For example, the MATPRO model was followed by a model by Lucuta that accounted for burnup degradation and was incorporated in FRAPCON-3. However, there were several issues with the Lucuta model, including its non-standard form and weak burnup degradation term, that led to its replacement by a modified model developed at NFI. Further refinements of this model were implemented by Pacific Northwest National Laboratory (PNNL) to provide a better fit to $\mathrm{UO}_{2}$ data. In addition, PNNL added refinements to include the Duriezderived modifications to the NFI model, which permit MOX fuels to be modeled. These updates were incorporated in FRAPCON-3.3 and continue to be used in FRAPCON-3.4.

\subsubsection{Model Development and Comparisons}

The original MATPRO model for fuel thermal conductivity is based on a mechanistic description of thermal conductivity, including lattice vibration (i.e., phonon) and electron-hole pair (i.e., electronic) contributions (Hagrman et al., 1981). The MATPRO thermal conductivity model is a function of temperature, density, $\mathrm{O} / \mathrm{M}$ ratio, and plutonium content. Burnup is also included; however, it is only used to calculate the melt temperature. Unlike the models used in 
FRAPCON-3.4 and FRAPTRAN 1.4, MATPRO does not account for the degradation of thermal conductivity with increasing burnup.

The dominant contributor to thermal conductivity (i.e., phonon or electron) is dependent on temperature. Phonon contributions to thermal conductivity are dominant below $1500 \mathrm{~K}$, whereas electronic contributions are dominant above $2000 \mathrm{~K}$. In general, thermal conductivity decreases with increasing porosity, but this effect is significantly decreased above $1600 \mathrm{~K}$ due to radiation effects and gas conductivity within the pores at these temperatures. At higher temperatures (> 3000K), estimates of liquid fuel thermal conductivity are made from physical considerations because data for molten fuel have not been found.

The MATPRO model used to express thermal conductivity of solid fuel is described with Equation 2.3-1.

$$
\begin{aligned}
& \mathrm{k}=\left(\frac{\mathrm{D}}{1+\left(6.5-0.00469 \mathrm{~T}^{\prime}\right)(1-\mathrm{D})}\right)\left(\frac{\mathrm{C}_{\mathrm{V}}}{\left(\mathrm{A}+\mathrm{BT}^{\prime \prime}\right)\left(1+3 \mathrm{e}_{\text {th }}\right)}\right) \ldots \ldots \\
& \ldots \ldots+5.2997 \times 10^{-3} \mathrm{~T}[\exp (-13358 / \mathrm{T})]\left[1+0.169[(13358 / \mathrm{T})+2]^{2}\right\}
\end{aligned}
$$

\begin{tabular}{|c|c|c|}
\hline $\mathrm{k}$ & $=$ & hermal conductivity (W/m*K). \\
\hline $\mathrm{D}$ & $=$ & fraction of theoretical density (unitless). \\
\hline $\mathrm{C}_{\mathrm{V}}$ & $=$ & $\begin{array}{l}\text { phonon contribution to the specific heat at constant volume }\left(\mathrm{J} / \mathrm{kg}^{*} \mathrm{~K}\right) \text {. The first } \\
\text { term of the correlation for fuel specific heat capacity is used for this factor. }\end{array}$ \\
\hline$e_{\text {th }}$ & $=$ & $\begin{array}{l}\text { linear strain caused by thermal expansion when temperature is }>300 \mathrm{~K} \text { (unitless); } \\
\text { the MATPRO correlation for fuel thermal expansion is used for this factor }\end{array}$ \\
\hline $\mathrm{T}$ & $=$ & fuel temperature (K). \\
\hline $\mathrm{T}^{\prime}$ & $=$ & $\begin{array}{l}\text { porosity correction for temperature, for temperature }<1364, \mathrm{~T}^{\prime}=6.50- \\
\mathrm{T}^{*}\left(4.69 \times 10^{-3}\right) \text {, for temperature }>1834, \mathrm{~T}^{\prime}=-1 \text {, and for temperatures in the range } \\
\text { from } 1364 \text { to } 1834 \mathrm{~K}, \mathrm{~T}^{\prime} \text { is found by interpolation. }\end{array}$ \\
\hline $\mathrm{T}^{\prime \prime}$ & $=$ & $\begin{array}{l}\text { fuel temperature if }<1800 \mathrm{~K} \text {. For temperatures }>2300 \mathrm{~K}, \mathrm{~T}^{\prime \prime} \text { is equal to } 2050 \mathrm{~K} \text {; } \\
\text { for temperature in the range } 1800 \text { to } 2300 \mathrm{~K}, \mathrm{~T}^{\prime \prime} \text { is found by interpolation. }\end{array}$ \\
\hline A & $=$ & $\begin{array}{l}\text { a factor proportional to the point defect contribution to the phonon mean free } \\
\text { path }\left(\mathrm{m}^{*} \mathrm{~s} / \mathrm{kg}^{*} \mathrm{~K}\right) \text {; the correlation used for this factor is } 0.339+12.6 \mathrm{x} \text { absolute } \\
\text { value }(2.0-\mathrm{O} / \mathrm{M} \text { ratio). }\end{array}$ \\
\hline$B$ & $=$ & $\begin{array}{l}\text { a factor proportional to the phonon-phonon scattering contribution to the phonon } \\
\text { mean free path }\left(\mathrm{m}^{*} \mathrm{~s} / \mathrm{kg} \mathrm{K}^{*} \mathrm{~K}\right) \text {; the correlation used for this factor is } 0.06867 \mathrm{x}(1+ \\
0.6238 \mathrm{x} \text { plutonium content of the fuel). }\end{array}$ \\
\hline
\end{tabular}

where

The first term of Equation 2.3-1 represents the phonon contribution to specific heat. The temperature and density dependence of this term can be seen in Equation 2.3-1. Although not as evident, the dependence of the first term on $\mathrm{O} / \mathrm{M}$ ratio and plutonium content is also included. The specific heat capacity of the fuel $\left(\mathrm{C}_{\mathrm{V}}\right)$ and the phonon scattering factor $(\mathrm{B})$ are dependent on the plutonium content, while the point defect contribution factor (A) is dependent on the $\mathrm{O} / \mathrm{M}$ ratio. Temperature dependence can be observed in the first and second terms of Equation 2.3-1, which represents the electronic contribution. 
This MATPRO model (Hagrman et al., 1981) was initially replaced in FRAPCON-3 and FRAPTRAN with a model from Lucuta (Lucuta et al., 1996) which accounts for the degradation of thermal conductivity due to burnup. The Lucuta model for fuel thermal conductivity, $\mathrm{K}$, is presented in Equation 2.3-2

where

$$
\mathrm{K}=\mathrm{K}_{\mathrm{o}} \bullet \mathrm{FD} \bullet \mathrm{FP} \bullet \mathrm{FM} \bullet \mathrm{FR}
$$

$\mathrm{K}_{\mathrm{o}} \quad=\quad$ thermal conductivity of unirradiated, fully dense urania

$\mathrm{FD} \quad=\quad$ factor for dissolved fission products

$\mathrm{FP} \quad=\quad$ factor for precipitated fission products

$\mathrm{FM}=$ factor to correct for the Maxwell porosity effect

$\mathrm{FR} \quad=\quad$ factor for the radiation effect

Thermal conductivity of unirradiated, fully dense urania and factors included in the Lucuta model are described by the Equations 2.3-3 through 2.3-7.

$$
\begin{gathered}
\mathrm{K}_{\mathrm{o}}=\frac{1}{0.0375+2.165 \times 10^{-4} \mathrm{~T}}+\left[\frac{4.715 \times 10^{9}}{\mathrm{~T}^{2}}\right] \exp \left[-\frac{16361}{\mathrm{~T}}\right] \\
\mathrm{FD}=\left[\frac{1.09}{\mathrm{~B}^{3.265}}+\frac{0.0643}{\sqrt{\mathrm{B}}} \sqrt{\mathrm{T}}\right] \arctan \left[\frac{1}{\left.\frac{1.09}{\mathrm{~B}^{3.265}}+\frac{0.0643}{\sqrt{\mathrm{B}}} \sqrt{\mathrm{T}}\right]}\right] \\
\mathrm{FP}=1+\left[\frac{0.019 \mathrm{~B}}{3-0.019 \mathrm{~B}}\right]\left[\frac{1}{1+\exp \left(-\frac{\mathrm{T}-1200}{100}\right)}\right] \\
\mathrm{FM}=\frac{1-\mathrm{p}}{1+(\mathrm{s}-1) \mathrm{p}} \\
\mathrm{FR}=1-\frac{0.2}{1+\exp \left(\frac{\mathrm{T}-900}{80}\right)}
\end{gathered}
$$

where

$\mathrm{K}_{\mathrm{o}}=$ conductivity of unirradiated, fully dense urania (W/m-K)

$\mathrm{T}=$ temperature $(\mathrm{K})$

$\mathrm{B}=\quad$ burnup in atom\% $(1$ atom $\%=9.383 \mathrm{GWd} / \mathrm{MTU}$ at $200 \mathrm{MeV} /$ fission $)$

$\mathrm{p} \quad=\quad$ porosity fraction (as-fabricated plus swelling)

$\mathrm{s} \quad=\quad$ shape factor $(=1.5$ for spherical pores $)$

Although the Lucuta model for thermal conductivity accounted for burnup degradation, this model tended to overpredict thermal conductivity values at high temperature ( $>2200 \mathrm{~K}$ ). 
Furthermore, the model had too little burnup degradation, making it non-conservative above $30 \mathrm{GWd} / \mathrm{MTU}$. In addition, the form of the Lucuta model is non-standard, which does not facilitate comparison between models. As a result, the Lucuta model was later replaced with a modified version of a thermal conductivity model proposed by NFI (Ohira and Itagaki, 1997) (Lanning et al., 2005).

Both FRAPCON-3.4 and FRAPTRAN 1.4 currently model urania fuel pellet thermal conductivity with the modified version of the pellet thermal conductivity model proposed by NFI (Ohira and Itagaki, 1997). The original NFI model was modified to alter the temperature-dependent portion of the burnup function in the phonon terms and change the electronic term (Lanning et al., 2005). The original (unmodified) and modified versions of the NFI thermal conductivity model are presented in Equations 2.3-8 and 2.3-9, respectively.

$$
\begin{gathered}
\mathrm{K}_{95}=\frac{1}{\mathrm{~A}+\mathrm{BT}+\mathrm{f}(\mathrm{Bu})+\mathrm{g}(\mathrm{Bu}) \mathrm{h}(\mathrm{T})}-\mathrm{CT}^{2}+\mathrm{DT}^{4} \\
\mathrm{~K}_{95}=\frac{1}{\mathrm{~A}+\mathrm{BT}+\mathrm{f}(\mathrm{Bu})+(1-0.9 \exp (-0.04 \mathrm{Bu})) \mathrm{g}(\mathrm{Bu}) \mathrm{h}(\mathrm{T})}+\frac{\mathrm{E}}{\mathrm{T}^{2}} \exp (-\mathrm{F} / \mathrm{T})
\end{gathered}
$$

where

$\begin{array}{lll}\mathrm{K} & = & \text { thermal conductivity, W/m-K } \\ \mathrm{T} & = & \text { temperature, } \mathrm{K} \\ \mathrm{Bu} & = & \text { burnup, GWd/MTU } \\ \mathrm{f}(\mathrm{Bu}) & = & \text { effect of fission products in crystal matrix (solution) } \\ \mathrm{g}(\mathrm{Bu}) & = & 0.00187^{*} \mathrm{Bu} \\ & = & \text { effect of irradiation defects } \\ \mathrm{h}(\mathrm{T}) & = & \text { temperature dependence of annealing on irradiation defects } \\ & = & 1 \\ \mathrm{Q} & = & 1+396 \mathrm{exp}(-\mathrm{Q} / \mathrm{T}) \\ \mathrm{A} & = & 0.0452 \mathrm{~m}-\mathrm{K} / \mathrm{W} \\ \mathrm{B} & = & 2.46 \times 10^{-4} \mathrm{~m}-\mathrm{K} / \mathrm{W} / \mathrm{K} \\ \mathrm{C} & = & 5.47 \times 10^{-9} \mathrm{~W} / \mathrm{m}-\mathrm{K}^{3} \\ \mathrm{D} & = & 2.29 \times 10^{14} \mathrm{~W} / \mathrm{m}-\mathrm{K}^{5} \\ \mathrm{E} & = & 3.5 \times 10^{9} \mathrm{~W}-\mathrm{K} / \mathrm{m} \\ \mathrm{F} & = & 16,361 \mathrm{~K}\end{array}$

The NFI model was further modified in FRAPCON-3.4 with a data correlation presented by Massih (Massih et al., 1992) to include gadolinia content. The modified NFI with gadolinia dependency used in FRAPCON-3.4 is presented in Equation 2.3-10.

$$
\begin{aligned}
& \mathrm{K}=\frac{1}{\mathrm{~A}+\mathrm{a} \cdot \mathrm{gad}+\mathrm{BT}+\mathrm{f}(\mathrm{Bu})+(1-0.9 \exp (-0.04 \mathrm{Bu})) \mathrm{g}(\mathrm{Bu}) \mathrm{h}(\mathrm{T})}+\cdots \\
& \ldots \frac{\mathrm{E}}{\mathrm{T}^{2}} \exp (-\mathrm{F} / \mathrm{T})
\end{aligned}
$$


where

$\begin{array}{llll}\mathrm{a} & = & \text { constant } & = \\ \text { gad } & = & \text { weight fraction of gadolinia }\end{array}$

As applied in FRACON-3.4 and FRAPTRAN 1.4, the above models are adjusted for as-fabricated fuel density (in fraction of theoretical density [TD]) using the Lucuta recommendation for spherical-shaped pores (Lucuta et al., 1996), as follows:

where

$$
\mathrm{K}_{\mathrm{d}}=1.0789 * \mathrm{~K}_{95} *[\mathrm{~d} /\{1.0+0.5(1-\mathrm{d})\}]
$$

$\mathrm{K}_{\mathrm{d}} \quad=\quad$ thermal conductivity adjusted for as-fabricated fuel density, $\mathrm{d}$

$\mathrm{K}_{95}=\quad$ thermal conductivity for 95 percent dense fuel

$\mathrm{d}=$ as-fabricated fuel density

The phonon-term modification in the modified NFI model reduces defect annealing at low burnup from that by Ohira and Itagaki (NFI). However, the original temperature-dependent annealing is restored at higher burnups such that, for burnups greater than $40 \mathrm{GWd} / \mathrm{MTU}$, the phonon term is equivalent to that in the original NFI model. The electronic terms (which in either case become significant above $1500 \mathrm{~K}$ ) are altered in the modified NFI model to a more theoretically based equation. The magnitude is slightly lower than the original model at high temperature. This adjustment was indicated by the Institute for Transuranium Elements data on unirradiated pressurized-water reactor (PWR) pellet material at temperatures approaching fuel melting (Ronchi et al., 1999).

At low burnups ( $<20 \mathrm{GWd} / \mathrm{MTU})$ and low temperatures $(<1000 \mathrm{~K})$, the modified model is higher than the unmodified NFI model and roughly equivalent to the Lucuta model without its radiation term. At higher burnup ( $>30 \mathrm{GWd} / \mathrm{MTU}$ ), the modified model is equivalent to the original NFI model with the exception of the small reduction at very high temperatures.

Fuel thermal conductivity modeling was extended to include MOX in FRAPCON-3 and FRAPTRAN by adopting a model that was a combination of the Duriez stoichiometry-dependent correlation, which is derived from diffusivity measurements on unirradiated fuel pellets, and the modified version of the NFI model, which includes burnup degradation effects. The Duriez data places greater dependence of MOX thermal conductivity on stoichiometry, but only a minor dependence on plutonia content (Duriez et al., 2000). The Duriez/modified NFI model is presented in Equation 2.3-12.

$$
\begin{aligned}
K_{95}= & \frac{1}{A(x)+a \cdot \operatorname{gad}+B(x) T+f(B u)+(1-0.9 \exp (-0.4 B u)) g(B u) h(T)} \ldots \\
& \ldots+\frac{C_{\text {mod }}}{T^{2}} \exp \left(-\frac{D}{T}\right)
\end{aligned}
$$

where

$\mathrm{K}_{95}=\quad$ conductivity at 95 percent $\mathrm{TD}, \mathrm{W} / \mathrm{m}-\mathrm{K}$

$\mathrm{x}=2.00-\mathrm{O} / \mathrm{M}$ (i.e., oxygen-to-metal ratio)

$\mathrm{T}=$ temperature, $\mathrm{K}$

$\mathrm{A}(\mathrm{x})=2.85 \mathrm{x}+0.035, \mathrm{~m}-\mathrm{K} / \mathrm{W}$ 


$\begin{array}{lll}\mathrm{B}(\mathrm{x}) & = & (2.86-7.15 \mathrm{x}) * 1 \mathrm{E}-4 \mathrm{~m} / \mathrm{W} \\ \mathrm{C} & = & 1.689 \times 10^{9}, \mathrm{~W}-\mathrm{K} / \mathrm{m} \\ \mathrm{D} & = & 13,520 \mathrm{~K} \\ \mathrm{a} & = & 1.1599 \\ \mathrm{gad} & = & \text { weight fraction gadolinia (not expected in MOX) } \\ \mathrm{Bu} & = & \text { burnup in } \mathrm{GW} / \mathrm{tHM} \\ \mathrm{f}(\mathrm{Bu}) & = & \text { effect of fission products in crystal matrix (solution) } \\ & = & 0.00187^{*} \mathrm{Bu} \\ \mathrm{g}(\mathrm{Bu}) & = & \text { effect of irradiation defects } \\ & = & 0.038^{0} \mathrm{Bu} .28 \\ \mathrm{~h}(\mathrm{~T}) & = & \text { temperature dependence of annealing on irradiation defects } \\ & = & 1 \\ \mathrm{Q} & = & \text { temperature dependent parameter (“Q } \mathrm{Q} / \mathrm{R} \text { ”) }=6380 \mathrm{~K} \\ \mathrm{C}_{\mathrm{mod}} & = & 1.5 \times 10^{9} \mathrm{~W}-\mathrm{K} / \mathrm{m}\end{array}$

FRAPCON 3 provides an option to use a fuel thermal conductivity model derived at Halden. The Halden correlation does not include dependency on stoichiometry, but does emphasize burnup effects. The Halden correlation tends to overpredict thermal conductivity data at high temperatures (> 2500K) (Lanning et al., 2005).

$$
\begin{aligned}
\mathrm{K}_{95}= & \frac{0.92}{0.1148+\mathrm{a} \cdot \operatorname{gad}+1.1599 \mathrm{x}+0.0040 \mathrm{~B}+2.475 \times 10^{-4}(1-0.00333 \mathrm{~B}) \Phi} \ldots \\
& \ldots+0.0132 \exp (0.00188 \mathrm{~T})
\end{aligned}
$$

where

$\begin{array}{lll}\mathrm{K}_{95} & = & \text { conductivity at } 95 \text { percent } \mathrm{TD}, \mathrm{W} / \mathrm{m}-\mathrm{K} \\ \mathrm{T} & = & \text { temperature, }{ }^{\circ} \mathrm{C} \\ \mathrm{a} & = & 1.1599 \\ \text { gad } & = & \text { weight fraction gadolinia (not expected in } \mathrm{MOX} \text { ) } \\ \mathrm{B} & = & \text { burnup in } \mathrm{MWd} / \mathrm{kg} \mathrm{UO}_{2} \\ \Phi & = & \text { minimum of } 1650^{\circ} \mathrm{C} \text { or current temperature in }{ }^{\circ} \mathrm{C}\end{array}$

To summarize, the modified NFI model is used for $\mathrm{UO}_{2}$, whereas the Duriez/NFI model is used for MOX. The modified NFI model used in FRAPCON-3.4 accounts for gad, but the modified NFI model in FRAPTRAN 1.4 does not. The Duriez/NFI models account for gad in both codes, although no gad additions are included in MOX at this time. Finally, FRAPCON-3.4 has the option for Halden thermal conductivity correlation, but FRAPTRAN does not.

A comparison between the MATPRO model and the modified NFI model is presented in Figure 2.3.3-1. The MATPRO model predicts lower thermal conductivities than the modified NFI model across the applicable temperature range (i.e., 500 to $3000 \mathrm{~K}$ ) for unirradiated $\mathrm{UO}_{2}$. However, unlike the MATPRO model, the modified NFI model can account for degradation of thermal conductivity with increasing burnup. The modified NFI model at a burnup level of $30 \mathrm{GWd} / \mathrm{MTU}$ is shown for comparison and reveals significant degradation of thermal conductivity. This represents an improvement relative to the MATPRO model, which does not account for burnup degradation of thermal conductivity. 


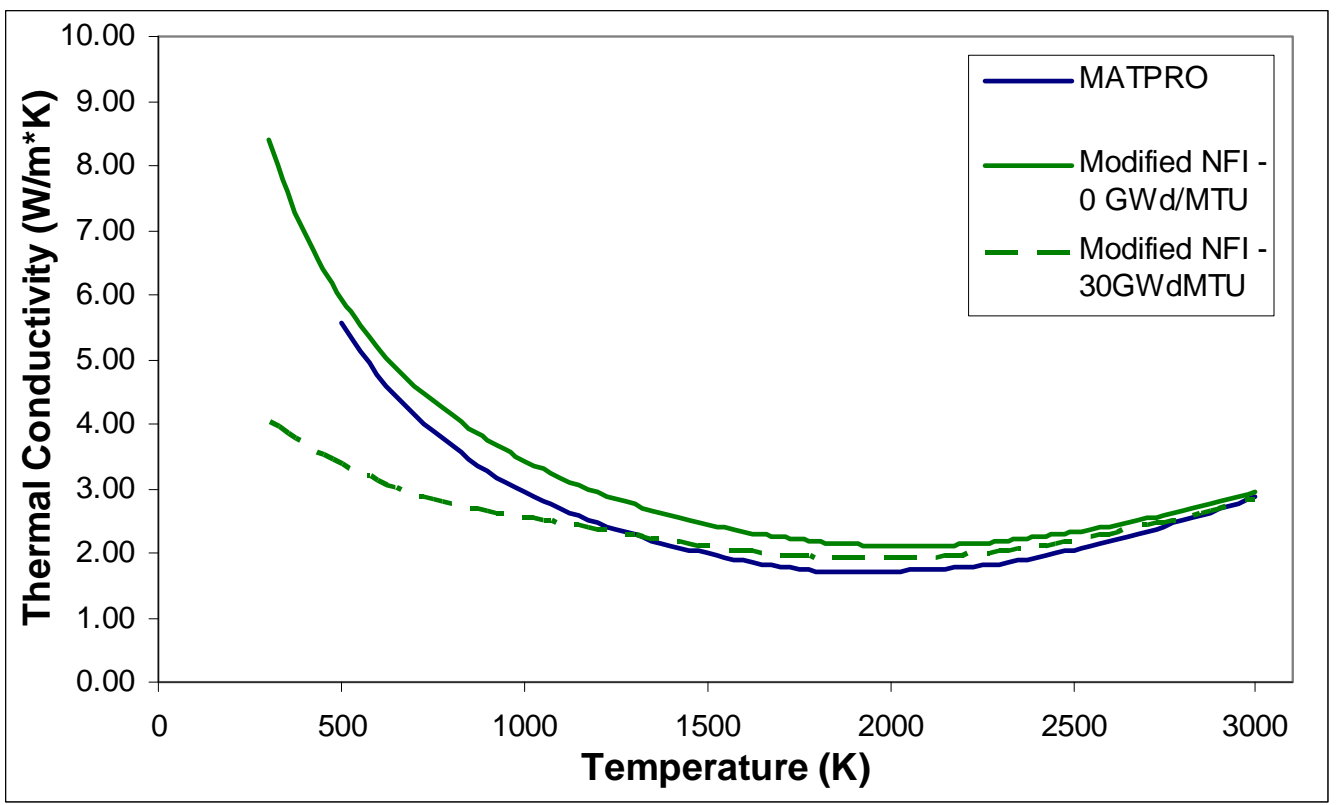

Figure 2.3.3-1 MATPRO and modified NFI thermal conductivity models for $\mathrm{UO}_{2}$ as a function of temperature. Unlike the MATPRO model, the modified NFI model can account for burnup and is presented at burnup levels of 0 and $30 \mathrm{GWd} / \mathrm{MTU}$ for comparison.

Model-to-data comparisons for the MATPRO and modified NFI models and data collected from unirradiated $\mathrm{UO}_{2}$ are made in Figures 2.3.3-2 through 2.3.3-3. In addition to Ronchi (1999), the unirradiated $\mathrm{UO}_{2}$ data sets from that were used to derive the MATPRO model are also included. Based on Figure 2.3.3-2, it can be seen that the MATPRO model tends to underpredict the thermal conductivity of the unirradiated $\mathrm{UO}_{2}$. The average bias in Figure 2.3.3-2 underpredicts thermal conductivity by about 9.5 percent, with a standard deviation of about 11 percent. The modified NFI model also underpredicts the unirradiated data, but only by about 2.4 percent and with a standard deviation of 8 percent (see Figure 2.3.3-3). Based on the smaller bias and standard deviation, it can be seen that the modified NFI model predicts the thermal conductivity of $\mathrm{UO}_{2}$ much better than the MATPRO model. 


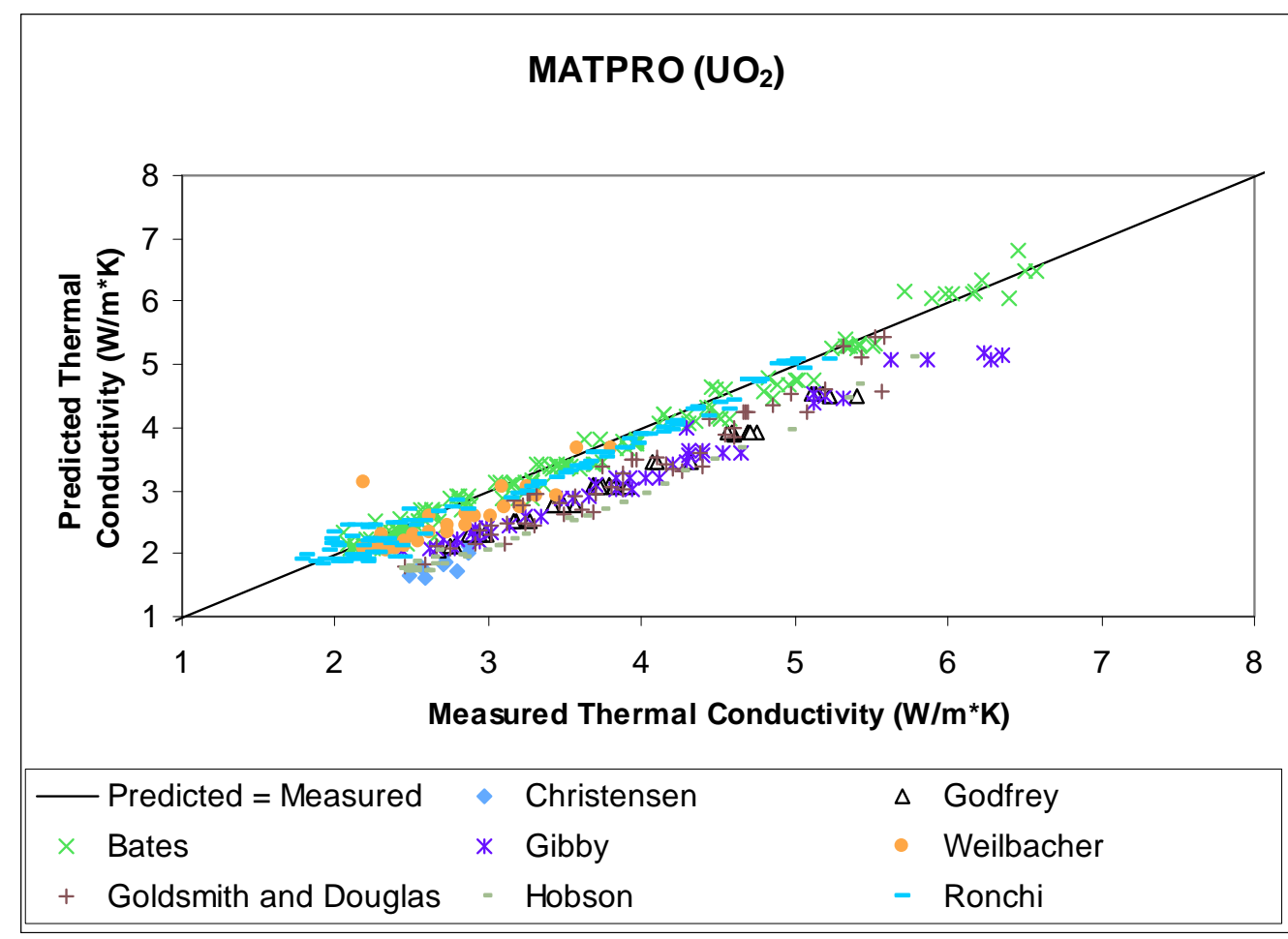

Figure 2.3.3-2 MATPRO model-to-data comparison for thermal conductivity of unirradiated $\mathrm{UO}_{2}$.

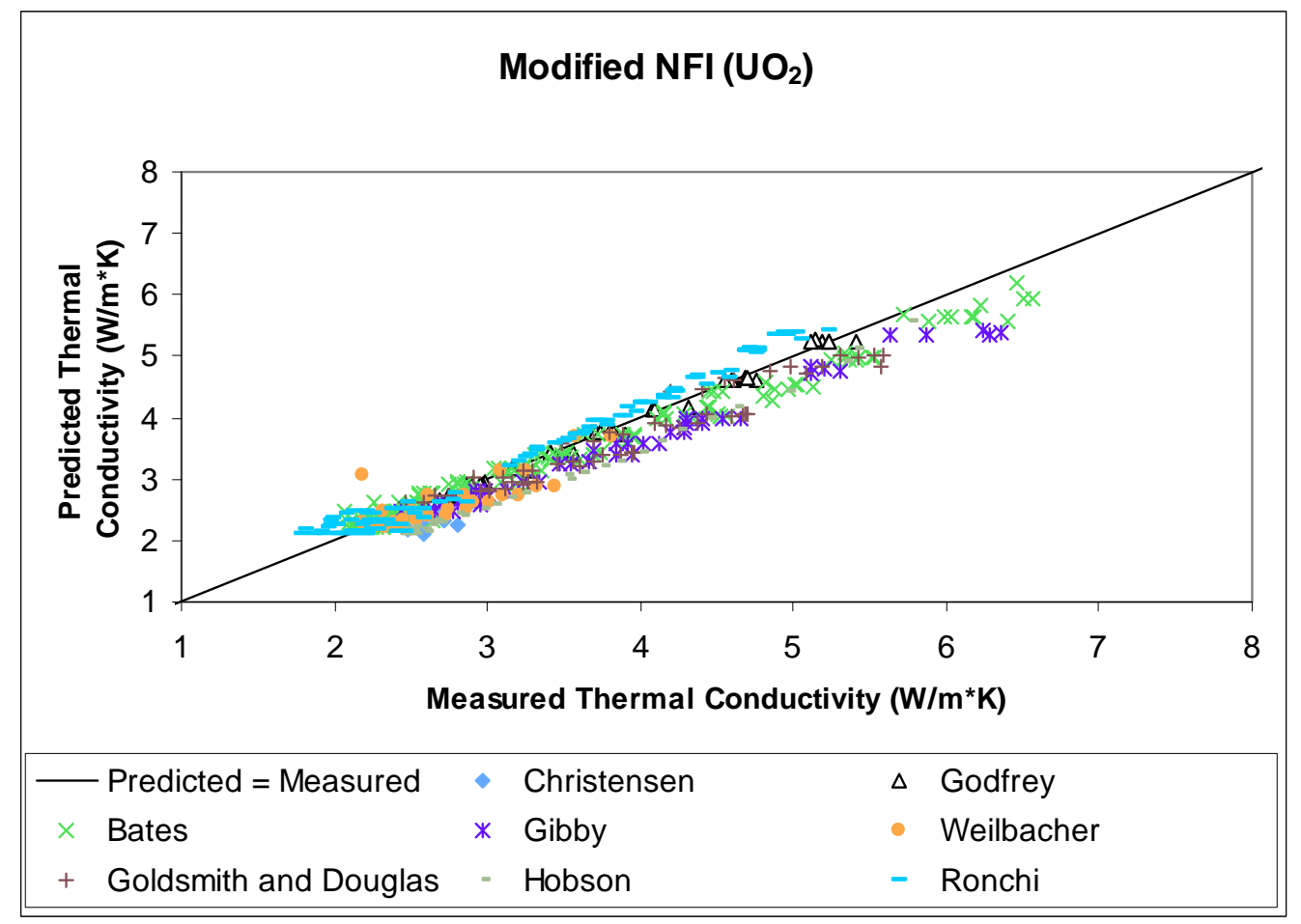

Figure 2.3.3-3 Modified NFI model-to-data comparison for thermal conductivity of unirradiated $\mathrm{UO}_{2}$. 
Model-to-data comparisons for the MATPRO and modified NFI models and data collected from irradiated $\mathrm{UO}_{2}$ are made in Figures 2.3.3-4 through 2.3.3-5. Based on Figure 2.3.3-4, it can be seen that the MATPRO model predicts significantly higher values for thermal conductivity than the measured values obtained from irradiated $\mathrm{UO}_{2}$. The average bias in Figure 2.3.3-4 indicates that the thermal conductivity is overpredicted by about 40 percent, with a standard deviation of about 31 percent. The modified NFI model also tends to overpredict the small amount of irradiated data on average, but only by about 5 percent and with a standard deviation of about 8 percent (see Figure 2.3.3-5). Based on the significantly smaller bias and standard deviation, it can be seen that the modified NFI model predicts the thermal conductivity of irradiated $\mathrm{UO}_{2}$ better than the MATPRO model. The disparity between models is attributed to the degradation of thermal conductivity with increasing burnup, which is accounted for in the modified NFI model but not the MATPRO model.

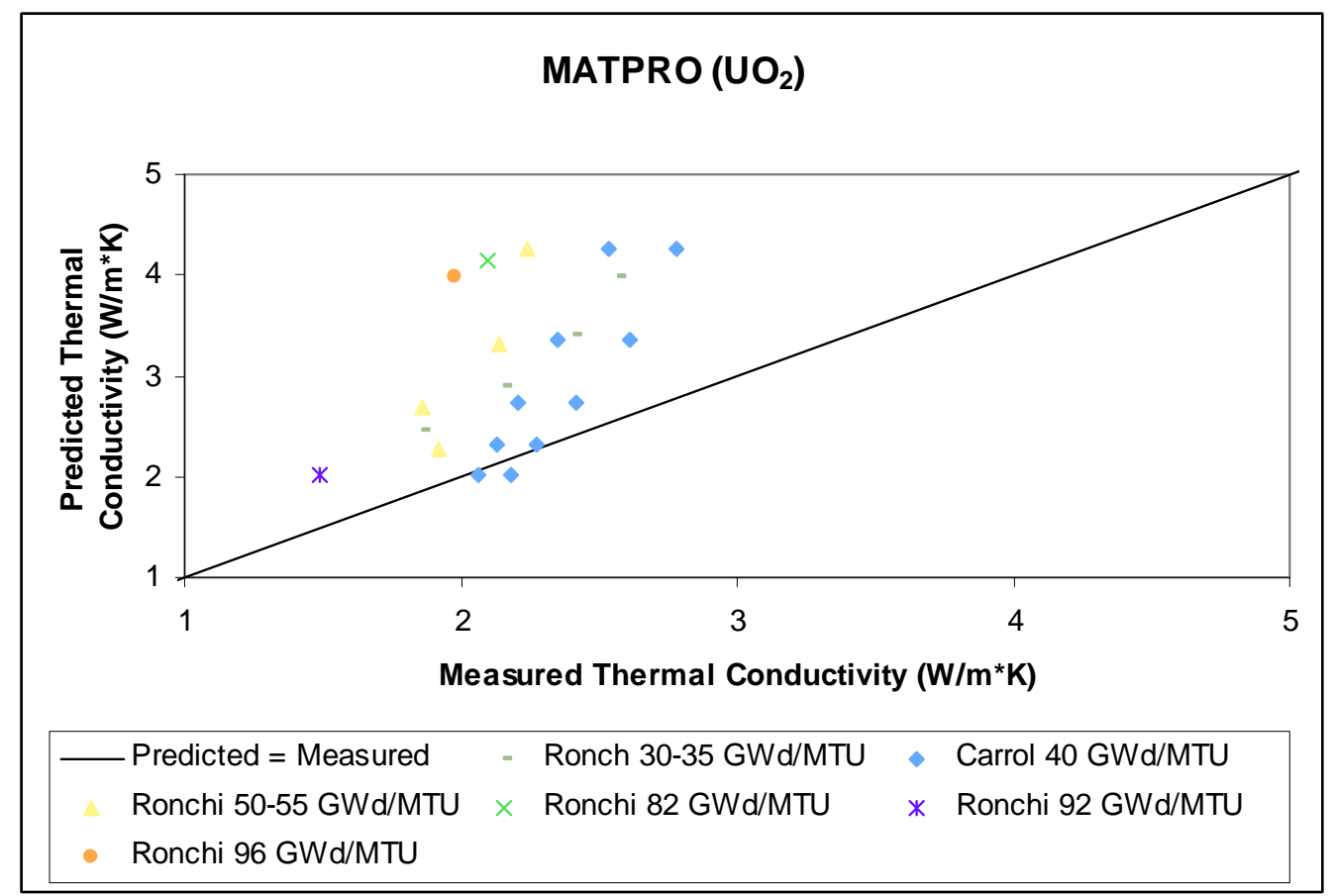

Figure 2.3.3-4 MATPRO model-to-data comparison for thermal conductivity of irradiated $\mathrm{UO}_{2}$. 


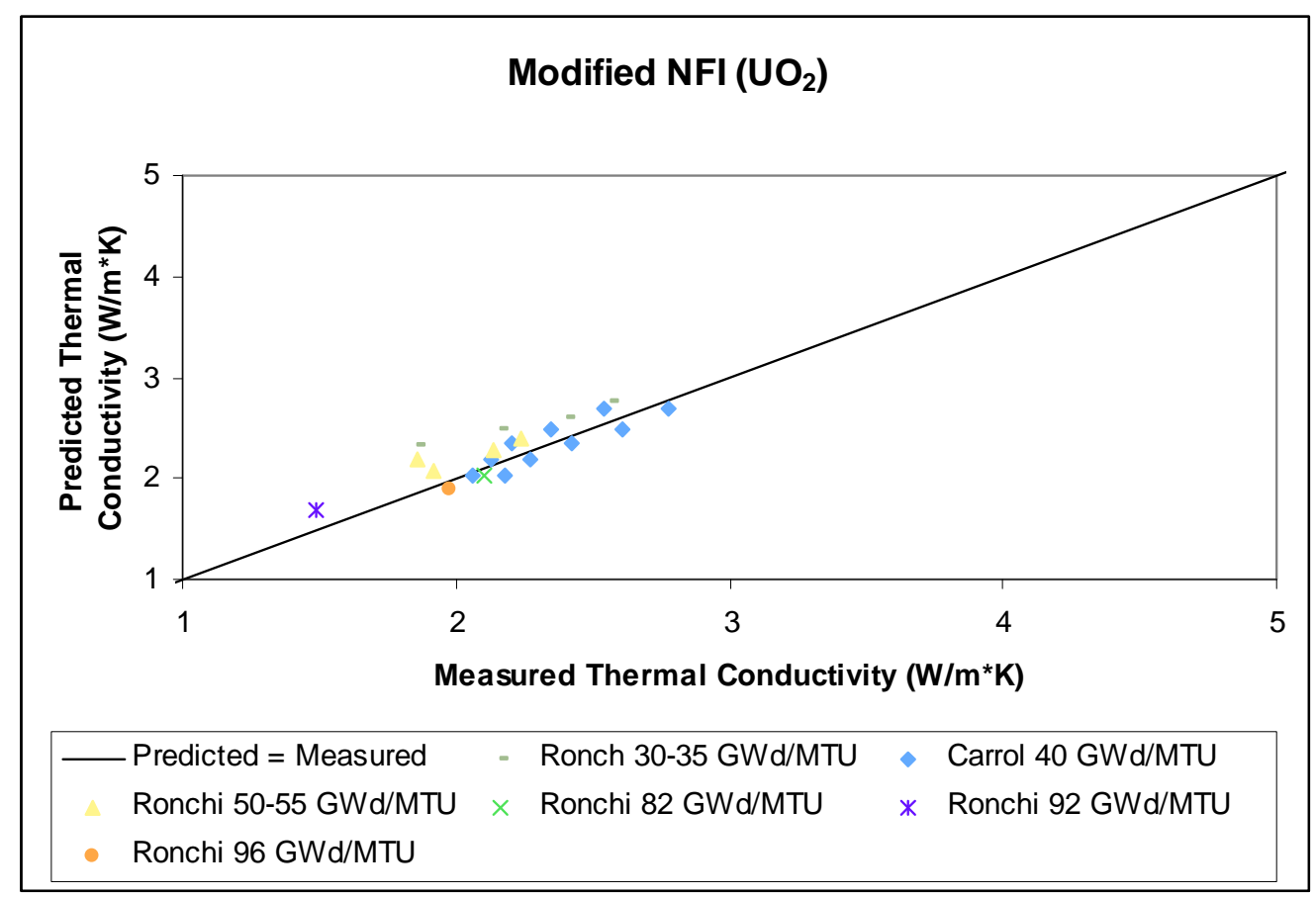

Figure 2.3.3-5 Modified NFI model-to-data comparison for thermal conductivity of irradiated $\mathrm{UO}_{2}$.

A comparison between the MATPRO model and the Duriez/modified NFI model is presented in Figure 2.3.3-6 for MOX fuel with a plutonium concentration of $7 \mathrm{wt} \%$. The MATPRO model predicts lower thermal conductivities than the Duriez/modified NFI model across the applicable temperature range (i.e., 500 to $3000 \mathrm{~K}$ ). However, unlike the MATPRO model, the Duriez/modified NFI model can account for degradation of thermal conductivity with increasing burnup. The Duriez/modified NFI model at a burnup level of $30 \mathrm{GWd} / \mathrm{MTU}$ is shown for comparison and reveals significant degradation of thermal conductivity. This represents an improvement relative to the MATPRO model, which does not account for burnup degradation of thermal conductivity. 


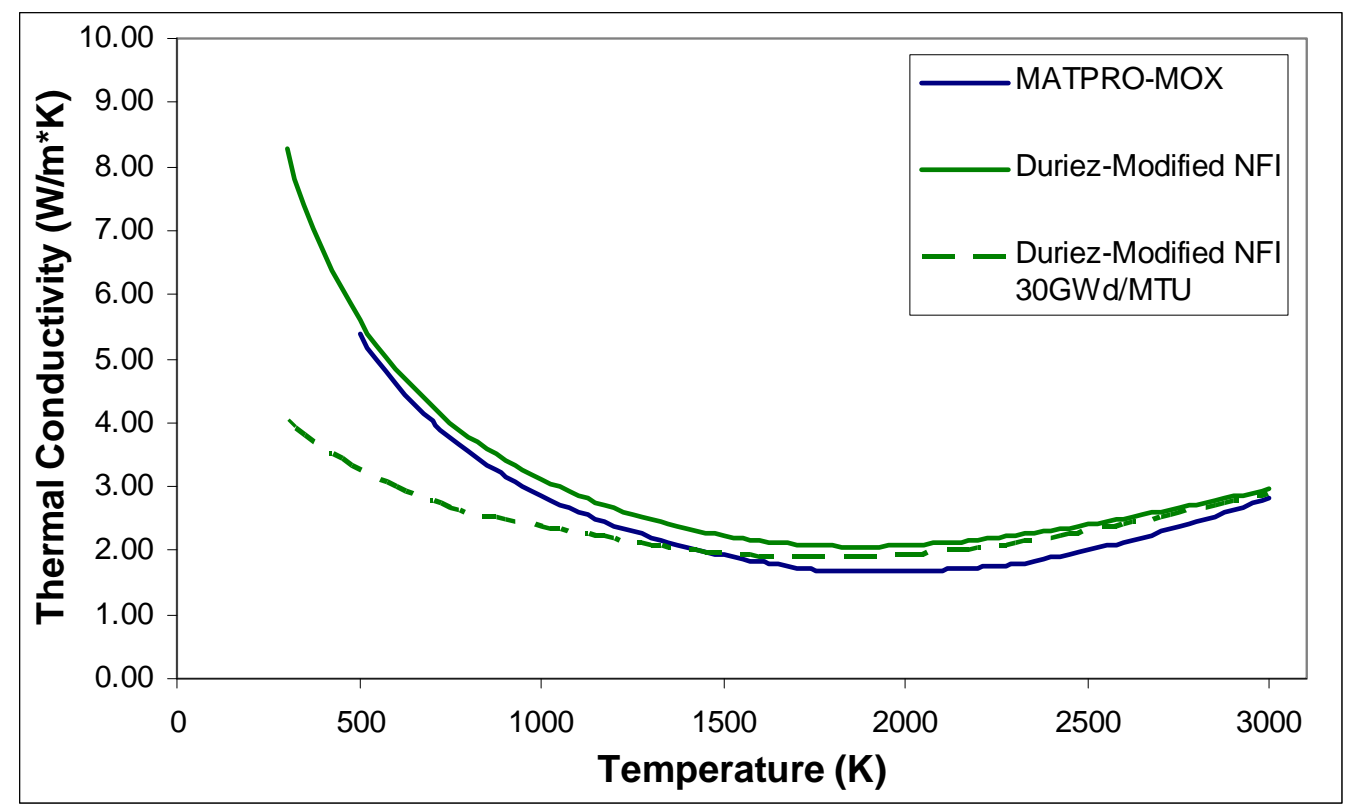

Figure 2.3.3-6 MATPRO and Duriez/modified NFI thermal conductivity models as a function of temperature for MOX fuel with 7 wt\% plutonium content. Unlike the MATPRO model, the Duriez/modified NFI model can account for burnup and is presented at burnup levels of 0 and $30 \mathrm{GWd} / \mathrm{MTU}$ for comparison.

Model-to-data comparisons for the MATPRO and Duriez/modified NFI models and unirradiated MOX fuel are made in Figures 2.3.3-7 and 2.3.3-8. The comparisons utilize the data used to derive the Duriez/modified NFI model. Data collected from MOX with plutonium concentrations of 3 and $15 \mathrm{wt} \%$ were used (Duriez et al., 2000). Based on Figure 2.3.3-7, it can be seen that the MATPRO model tends to underpredict thermal conductivity. The average bias in Figure 2.3.3-7 underpredicts thermal conductivity by about 9 percent at both 3 and $15 \mathrm{wt} \%$ plutonium concentrations. The standard deviation of the MATPRO predictive bias at both plutonium concentrations is under 6 percent. As expected, the Duriez/modified NFI model exhibits better agreement with the limited experimental data from which it was derived. The average bias indicates that the MOX model overpredicts by less than 2 percent and the standard deviation between the model and the data is less than 3 percent. Based on the smaller bias and standard deviation, it can be seen that the Duriez/modified NFI model fits this MOX thermal conductivity data better than the MATPRO model.

It should be noted that the MOX fuel thermal conductivity is strongly influenced by $\mathrm{O} / \mathrm{M}$ ratio, with conductivity decreasing with a deviation from stoichiometry (2.0). Based on Figure 2.3.3-8, it can be seen that plutonium content has a relatively minor effect at concentrations less than $15 \mathrm{wt} \%$. The current MOX fuel is fabricated closer to being stoichiometric than fuel typically fabricated more than 25 years ago. The older data from which the MATPRO model was developed were not as close to being stoichiometric as the more recent data. 


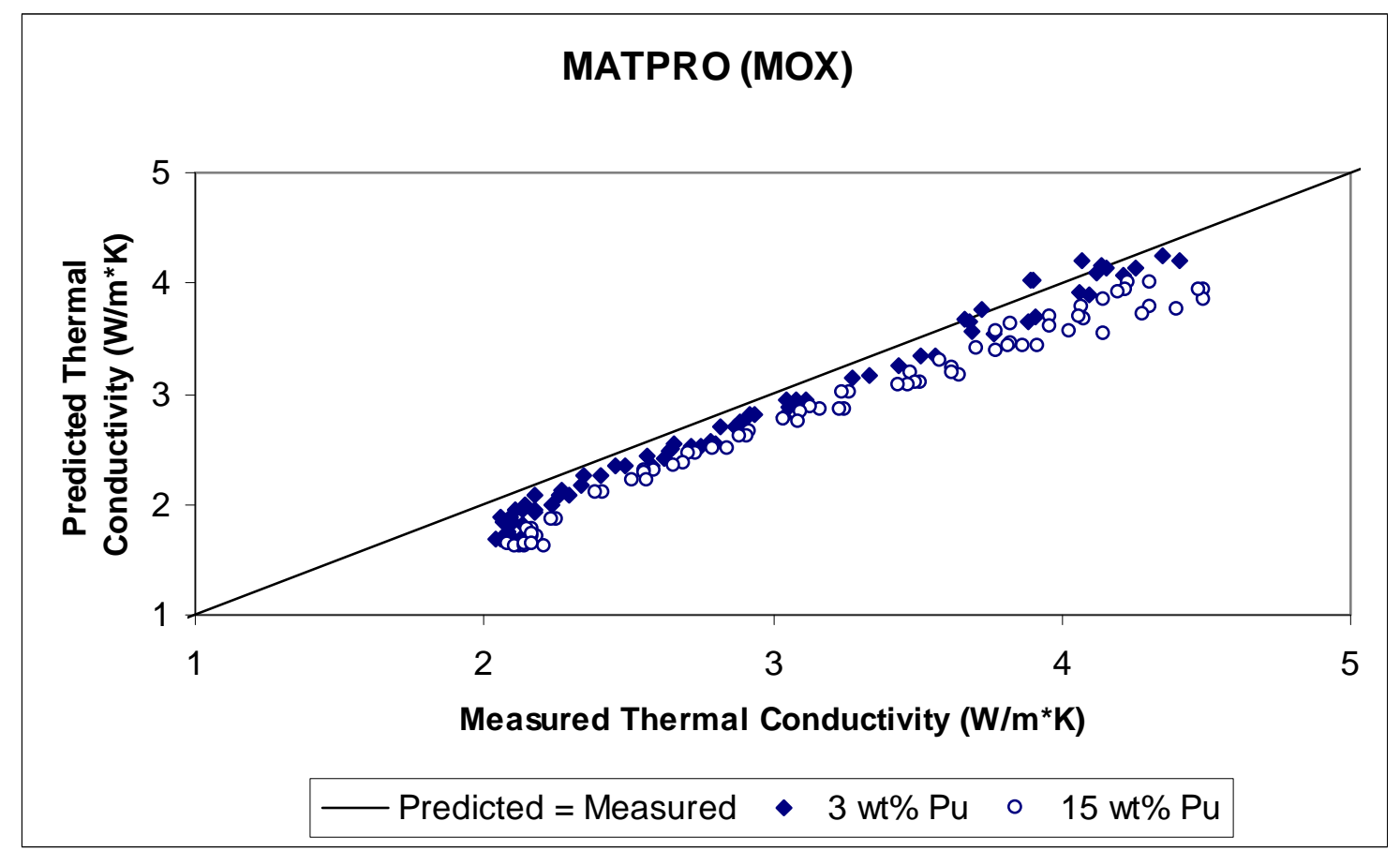

Figure 2.3.3-7 MATPRO model-to-data comparison for thermal conductivity of MOX modeled with $7 \mathrm{wt} \%$ of plutonium.

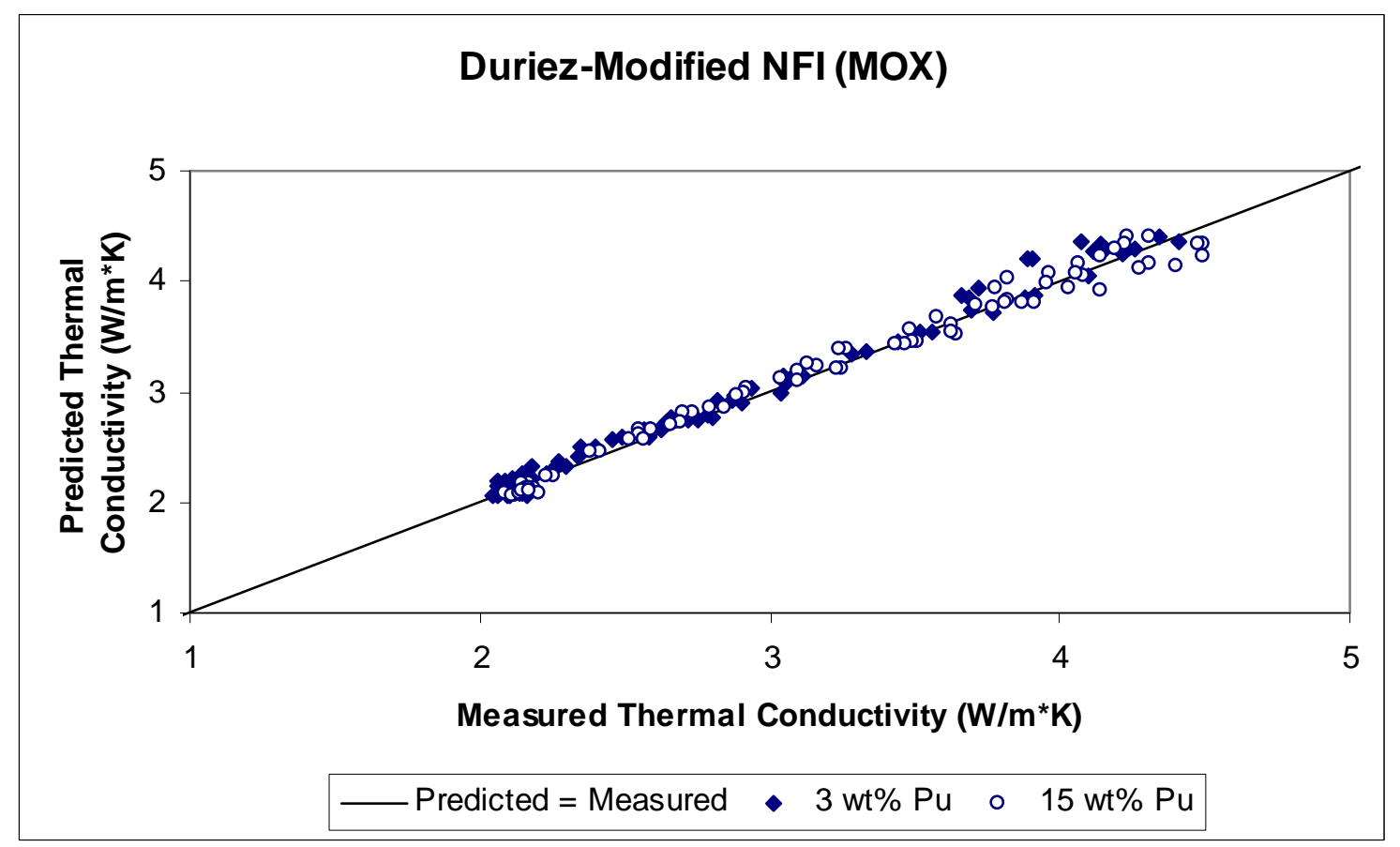

Figure 2.3.3-8 Duriez/modified NFI model-to-data comparison for thermal conductivity of MOX with modeled with $7 \mathrm{wt} \%$ of plutonium. 


\subsubsection{Applicability and Uncertainty}

The following are limits that restrict the applicability of the MATPRO fuel thermal conductivity model (Equation 2.3-1):

- Theoretical density must range between 90 and 100 percent.

- Temperature must range between 500 (the Debye temperature) and 3000K.

- Above $3000 \mathrm{~K}$, it is assumed that the fuel becomes molten and the uncertainties on predictions made above this temperature are large.

In addition, several assumptions have been made to provide an approximate model for effects of variation in the plutonium content and $\mathrm{O} / \mathrm{M}$ ratio of ceramic fuels:

- The effect of variation in theoretical density for MOX fuels has been assumed to be described by the porosity correction derived with $\mathrm{UO}_{2}$ data.

- The high-temperature electronic contribution to thermal conductivity has been assumed to be the same for $\mathrm{PuO}_{2}, \mathrm{UO}_{2}, \mathrm{UO}_{2}-\mathrm{Gd}_{2} \mathrm{O}_{3}$, and nonstoichiometric fuels.

- Variations in plutonium content have been assumed to affect only the phonon-phonon scattering factor.

- Variation in $\mathrm{O} / \mathrm{M}$ ratio has been assumed to affect only the defect term.

The bias and relative error of the MATPRO fuel thermal conductivity model was assessed based on the model-to-data comparisons previously presented for unirradiated $\mathrm{UO}_{2}$, irradiated $\mathrm{UO}_{2}$, and MOX. The average and standard deviations of the bias for unirradiated $\mathrm{UO}_{2}$ are approximately -9.5 and 11 percent, respectively, while the relative error is approximately 19 percent. For irradiated $\mathrm{UO}_{2}$, which has a limited number of data points for comparison, the average and standard deviations of the bias are 40 and 31 percent, respectively. The relative error for the limited number of irradiated $\mathrm{UO}_{2}$ data points is approximately 51 percent. Finally, for MOX, the average and standard deviations of the bias are -9 and 6 percent, respectively, while the relative error is approximately 13 percent. For reference, a negative bias indicates that the model underpredicts the data, whereas a positive bias indicates that the model overpredicts the data

For the modified NFI and the Duriez/modified NFI models, the recommended ranges for application for modeling the thermal conductivity of $\mathrm{UO}_{2}$ are as follows:

- Temperature: 300 to $3000 \mathrm{~K}$

- Rod-average burnup: 0 to $62 \mathrm{GWd} / \mathrm{MTU}$

- As-fabricated density: 92 to 97 percent TD

- Gadolinia content: 0 to $10 \mathrm{wt} \%$

For the Duriez/modified NFI model, the thermal conductivity model is applicable for plutonia particle sizes of less than 20 microns.

The bias and relative error of the modified NFI and the Duriez/modified NFI fuel thermal conductivity models were assessed based on the model-to-data comparisons previously presented for unirradiated $\mathrm{UO}_{2}$, irradiated $\mathrm{UO}_{2}$, and $\mathrm{MOX}$. The average and standard deviations of the bias for unirradiated $\mathrm{UO}_{2}$ are approximately -2.4 and 8 percent, respectively, while the relative error is approximately 9 percent. For irradiated $\mathrm{UO}_{2}$, which has a limited number of data points for 
comparison, the average and standard deviations of the bias are approximately 5 and 8 percent, respectively, while the relative error is approximately 10 percent. Finally, for the Duriez/modified NFI fuel thermal conductivity model for MOX, the average and standard deviations of the bias are less than 2 and 3 percent, respectively, while the relative error is approximately 3 percent. These are values are based on comparisons to the small amount of nearstoichiometric MOX data. For reference, a negative bias indicates that the model underpredicts the data, whereas a positive bias indicates that the model overpredicts the data

\subsubsection{References}

Hagrman, D.L., G.A. Reymann, and G.E. Mason. 1981. A Handbook of Materials Properties for Use in the Analysis of Light Water Reactor Fuel Rod Behavior, MATPRO Version 11 (Revision 2), NUREG/CR-0479 (TREE-1280), prepared by EG\&G Idaho, Inc., Idaho Falls, ID, for the U.S. Nuclear Regulatory Commission, Washington, D.C.

Lucuta, P.G., H.S. Matzke, and I.J. Hastings. 1996. “A Pragmatic Approach to Modeling Thermal Conductivity of Irradiated $\mathrm{UO}_{2}$ Fuel: Review and Recommendations,” Journal of Nuclear Materials, 232:166-180.

Ohira, K., and N. Itagaki. 1997. "Thermal Conductivity Measurements of High Burnup $\mathrm{UO}_{2}$ Pellet and a Benchmark Calculation of Fuel Center Temperature," in Proceedings of the ANS International Topical Meeting on LWR Fuel Performance, Portland, Oregon, March 2-6, 1997, pp. 541-549.

Lanning, D.D., C.E. Beyer, and K.G. Geelhood. 2005. FRAPCON-3 Updates, Including MixedOxide Fuel Properties, NUREG/CR-6534, Vol. 4, PNNL-11513, Pacific Northwest National Laboratory, Richland, WA.

Massih, A.R., S. Persson, and Z. Weiss. 1992. "Modeling of (U,Gd)O $\mathrm{O}_{2}$ Fuel Behavior in Boiling Water Reactors," in Proceedings of Symposium E on Nuclear Materials for Fission Reactors of the 1991 E-MRS Fall Conference; Journal of Nuclear Materials, 188:323-330.

Ronchi, C., M. Sheindlin, M. Musella, and G.J. Hyland. 1999. “Thermal Conductivity of Uranium Dioxide Up to 2900K from Simultaneous Measurement of the Heat Capacity and Thermal Diffusivity,” Journal of Applied Physics, 85(2):776-789.

Duriez, C., J.-P. Allesandri, T. Gervais, and Y. Philipponnea. 2000. "Thermal Conductivity of Hypostoichiometric Low Pu Content (U,Pu) $\mathrm{O}_{2-\mathrm{x}}$ Mixed Oxide,” Journal of Nuclear Materials, 277:143-158.

Bates, J.L., C.A. Hinman, and T. Kawada. 1967. "Electrical Conductivity of Uranium Dioxide," Journal of the American Ceramic Society, 50:652-656.

Goldsmith, L.A., and J.A.M. Douglas. 1973. "Measurements of the Thermal Conductivity of Uranium Dioxide at 670-1270 K,” Journal of Nuclear Materials, 47:31-42.

Christensen, J.A. et al. 1964. "Uranium Dioxide Thermal Conductivity,” Transactions of the American Nuclear Society, 7:391-392. 
Gibby, R.L. 1971. "The Effect of Plutonium Content on the Thermal Conductivity of (U,Pu) $\mathrm{O}_{2}$ Solid Solutions,” Journal of Nuclear Materials, 38:163-177.

Hobson, I.C., R. Taylor, and J.B. Ainscough. 1974. "Effect of Porosity and Stoichiometry on the Thermal Conductivity of Uranium Dioxide,” Journal of Physics, Section D: Applied Physics, 7:1003-1015.

Godfrey, T.G., et al. 1964. Thermal Conductivity of Uranium Dioxide and Armco Iron by an Improved Radial Heat Flow Technique, ORNL-3556, Oak Ridge National Laboratory, Oak Ridge, TN.

Weilbacher, J.C. 1972. “Diffusivite Thermique de l’Oxyde d’Uranium et de l’Oxyde de Thorium a Haute Temperature,” High Temperatures-High Pressure, 4:431-438.

Ronchi, C., M. Sheindlin, D. Staicu, and M. Kinoshita. 2004. "Effect of Burn-up on the Thermal Conductivity of Uranium Dioxide up to 100,000 $\mathrm{MWdt}^{-1}$," Journal of Nuclear Materials, 327:5876.

\subsection{Fuel Emissivity (FEMISS)}

The subroutine FEMISS is used to calculate the total hemispherical fuel emissivity (emissivity integrated over all wavelengths) as a function of temperature. Fuel emissivity is defined as the ratio of radiant energy emitted from a material to that emitted by a black body at the same temperature. The subroutine FEMISS is used to calculate radiant energy transfer from fuel to cladding in conjunction with thermal conduction. Radiant energy transfer can be a significant heat transfer mechanism, depending on the gap size, temperature gradient across the gap, and plenum gas. The FEMISS subroutine used by FRAPCON-3 and FRAPTRAN is the same as the subroutine documented in MATPRO.

\subsubsection{Model Development and Comparisons}

According to the Stefan-Boltzmann law, the total radiant power per unit area emitted by a body at temperature $\mathrm{T}$ is

$$
\mathrm{P}=\mathrm{e} \sigma \mathrm{T}^{4}
$$

where

$\mathrm{P} \quad=\quad$ radiant power per unit area $\left(\mathrm{W} / \mathrm{m}^{2}\right)$

e $\quad=\quad$ total hemispherical emissivity (unitless)

$\sigma \quad=\quad$ the Stefan-Boltzmann constant $\left(5.672 \times 10^{-8} \mathrm{~W} / \mathrm{m}^{2}-\mathrm{K}\right)$

$\mathrm{T} \quad=\quad$ temperature $(\mathrm{K})$

The expression used in the FEMISS subroutine to describe total emissivity is

$$
\mathrm{e}=0.78557+1.5263 \times 10^{-5} \mathrm{~T}
$$

The first term of Equation 2.4-2 is slightly less than the value used in MATPRO, 0.7856. The standard error of Equation 2.4-2 with respect to its database is \pm 6.796 percent, which is in 
agreement with the error reported in MATPRO, \pm 6.8 percent. These small differences are attributed to rounding error and do not significantly alter emissivity predictions.

The emissivity data were measured at temperatures up to approximately $2400 \mathrm{~K}$, and use of FEMISS above this temperature is speculative because of possible high-temperature effects that are not modeled. At the time of model development, there were no data to develop a MOX emissivity equation, so Equation 2.4-2 is also recommended for MOX.

The hemispherical spectral data of Held and Wilder (1969) and the emissivity data of Cabannes (1967) and J.M. Jones and D.G. Murchison (1965) were used in developing the FEMISS model. The emissivity data used to model comparison for FEMISS are presented in Figure 2.4-1.

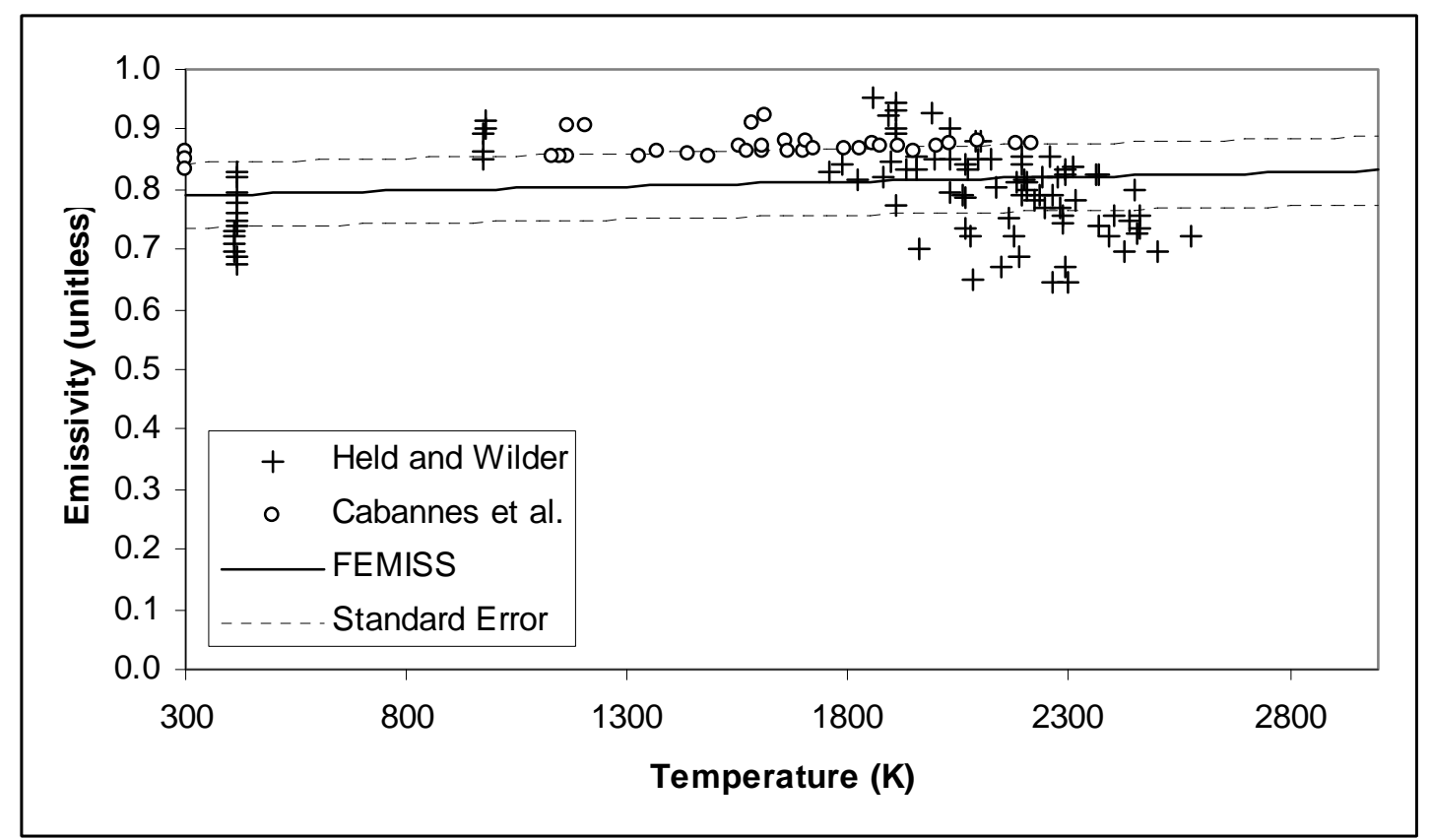

Figure 2.4-1 Data to model comparison for FEMISS.

\subsubsection{Applicability and Uncertainty}

The correlation used in the FEMISS subroutine to calculate fuel emissivity is applicable at temperatures up to $2400 \mathrm{~K}$. The standard error of this correlation with respect to the database from which it was derived is approximately \pm 6.8 percent

\subsubsection{References}

Held, P.C., and D.R. Wilder. 1969. "High Temperature Hemispherical Spectral Emittance of Uranium Oxides at 0.65 and $0.70 \mu \mathrm{m}$,” Journal of the American Ceramic Society, 52(4):182-185.

M.M.F. Cabannes, J.P. Stora, and J. Tsakiris. 1967. "ORTIQUE-MOLECULAIRE-Fracteurs de re'flexion et d'e'mission de $\mathrm{UO}_{2}$ a' haute Temperature,” C.R. Acad. SC. Paris, 264.

J.M. Jones and D.G. Murchison. 1965. “Optical Properties of Uranium Oxides,” Nature, 205(4972):663-665. 


\subsection{Fuel Thermal Expansion (FTHEXP)}

The subroutine FTHEXP models dimensional changes in unirradiated fuel pellets caused by thermal expansion. It can handle any combination of $\mathrm{UO}_{2}, \mathrm{UO}_{2}-\mathrm{Gd}_{2} \mathrm{O}_{3}$, or $\mathrm{PuO}_{2}$ in solid, liquid, or solid-liquid states and includes expansion due to the solid-liquid phase change. Dimensional changes in the fuel affect the pellet-to-cladding gap size, which is a major factor in determining gap heat transfer and thus the stored energy, an important quantity for safety analysis. The subroutine FTHEXP is used in FRAPCON-3.4, FRAPTRAN 1.4, and MATPRO. However, the FRAPCON-3 and FRAPTRAN codes use updated fitting coefficients based on more recent data.

\subsubsection{Model Development and Comparisons}

The FTHEXP subcode models fuel thermal expansion as a function of temperature, fraction of $\mathrm{PuO}_{2}$, and the fraction of fuel which is molten. The $\mathrm{O} / \mathrm{M}$ ratio is not included. When the departure from stoichiometry $(\mathrm{O} / \mathrm{M}-2.0)$ is greater than 0.2 , there is clearly an effect. However, this effect is ignored in modeling thermal expansion, since typical reactor fuels only deviate less than a tenth this much from the stoichiometric composition.

The equations for thermal expansion of $\mathrm{UO}_{2}, \mathrm{UO}_{2}-\mathrm{Gd}_{2} \mathrm{O}_{3}$, and $\mathrm{PuO}_{2}$ have the same form. In the solid phase, Equation 2.5-1 is used.

$$
\Delta \mathrm{L} / \mathrm{L}_{0}=\mathrm{K}_{1} \mathrm{~T}-\mathrm{K}_{2}+\mathrm{K}_{3} \exp \left(-\mathrm{E}_{\mathrm{D}} / \mathrm{kT}\right)
$$

where

$\begin{array}{lll}\Delta \mathrm{L} / \mathrm{L} & = & \text { linear strain caused by thermal expansion (equal to zero at 300K) (unitless) } \\ \mathrm{T} & = & \text { temperature }(\mathrm{K}) \\ \mathrm{E}_{\mathrm{D}} & = & \text { energy of formation of a defect }(\mathrm{J}) \\ \mathrm{k} & = & \text { Boltzmann's constant }\left(1.38 \times 10^{-23} \mathrm{~J} / \mathrm{K}\right)\end{array}$

and $\mathrm{K}_{1}, \mathrm{~K}_{2}$, and $\mathrm{K}_{3}$ are constants to be determined.

The correlation given in Equation 2.5-1 was originally used in MATPRO and was incorporated into both the FRAPCON-3 and FRAPTRAN codes. Data collected by Baldock et al. (1966), Burdick and Paker (1956), Gronvold (1955), Christensen (1963), and Kempeter and Elliott (1958) were used to determine the correlation constants for $\mathrm{UO}_{2}$ used in MATPRO. However, newer data provided by Martin (1988) and Momin et al. (1991) required the constants to be updated to improve the fit between the correlation and high-temperature data. These updated constants are included in FRAPCON-3.4/FRAPTRAN 1.4 (FRAP). Table 2.5-1 presents the original MATPRO constants and the updated constants for $\mathrm{UO}_{2}$, as well as the constants for $\mathrm{PuO}_{2}$.

Table 2.5-1 Parameters used in $\mathrm{UO}_{2}$ and $\mathrm{PuO}_{2}$ solid-phase thermal expansion correlations.

\begin{tabular}{|c|c|c|c|c|}
\hline Constant & $\begin{array}{c}\text { MATPRO } \\
\mathbf{U O}_{2}\end{array}$ & $\begin{array}{c}\text { FRAP } \\
\mathbf{U O}_{2}\end{array}$ & PuO $_{2}$ & Units \\
\hline $\mathrm{K}_{1}$ & $1.0 \times 10^{-5}$ & $9.80 \times 10^{-6}$ & $9.0 \times 10^{-6}$ & $\mathrm{~K}^{-1}$ \\
\hline $\mathrm{K}_{2}$ & $3.0 \times 10^{-3}$ & $2.61 \times 10^{-3}$ & $2.7 \times 10^{-3}$ & Unitless \\
\hline $\mathrm{K}_{3}$ & $4.0 \times 10^{-2}$ & $3.16 \times 10^{-1}$ & $7.0 \times 10^{-2}$ & Unitless \\
\hline $\mathrm{E}_{\mathrm{D}}$ & $6.9 \times 10^{-20}$ & $1.32 \times 10^{-19}$ & $7.0 \times 10^{-20}$ & $\mathrm{~J}$ \\
\hline
\end{tabular}


For mixed $\mathrm{UO}_{2}$ and $\mathrm{PuO}_{2}$, the thermal expansion of the solid is found by combining the contribution from each constituent in proportion to its weight fraction.

During melting, an expansion equal to a linear strain of 0.043 occurs. If the fuel is partially molten, the strain due to thermal expansion is given by Equation 2.5-2.

where

$$
\Delta \mathrm{L} / \mathrm{L}_{0}=\Delta \mathrm{L} / \mathrm{L}_{0}\left(\mathrm{~T}_{\mathrm{m}}\right)+0.043 \cdot \mathrm{FACMOT}
$$

$\Delta \mathrm{L} / \mathrm{L}_{0}\left(\mathrm{~T}_{\mathrm{m}}\right) \quad=\quad$ thermal expansion strain of solid fuel from equations with $\mathrm{T}=\mathrm{T}_{\mathrm{m}}$

$\mathrm{T}_{\mathrm{m}} \quad=\quad$ melting temperature of the fuel (K)

FACMOT $=$ fraction of the fuel which is molten (unitless)

If FACMOT $=0.0$, the fuel is all solid;

If FACMOT $=1.0$, the fuel is all molten

The correlation used to describe the expansion of entirely molten fuel is given by Equation 2.5-3.

$$
\Delta \mathrm{L} / \mathrm{L}_{0}=\Delta \mathrm{L} / \mathrm{L}_{0}\left(\mathrm{~T}_{\mathrm{m}}\right)+0.043+3.6 \times 10^{-5}\left[\mathrm{~T}-\left(\mathrm{T}_{\mathrm{m}}+\Delta \mathrm{T}_{\mathrm{m}}\right)\right]
$$

The solid-to-liquid phase transition is isothermal only for pure $\mathrm{UO}_{2}$ or pure $\mathrm{PuO}_{2}$. For $\mathrm{MOX}$, the transition occurs over a finite temperature range, denoted in Equation 2.5-3 by $\Delta \mathrm{T}_{\mathrm{m}}$.

Comparisons between the databases for $\mathrm{UO}_{2}$ and $\mathrm{PuO}_{2}$ with their respective correlations are presented in Figures 2.5-1 and 2.5-2, respectively. In Figure 2.5-1, it can be seen that the updated constants used in FRAPCON-3.4/FRAPTRAN 1.4 (FRAP) for $\mathrm{UO}_{2}$ provide a better fit to the high-temperature data than the previous constants used in MATPRO. The comparison in Figure 2.5-2 reveals good agreement between the correlation and the database.

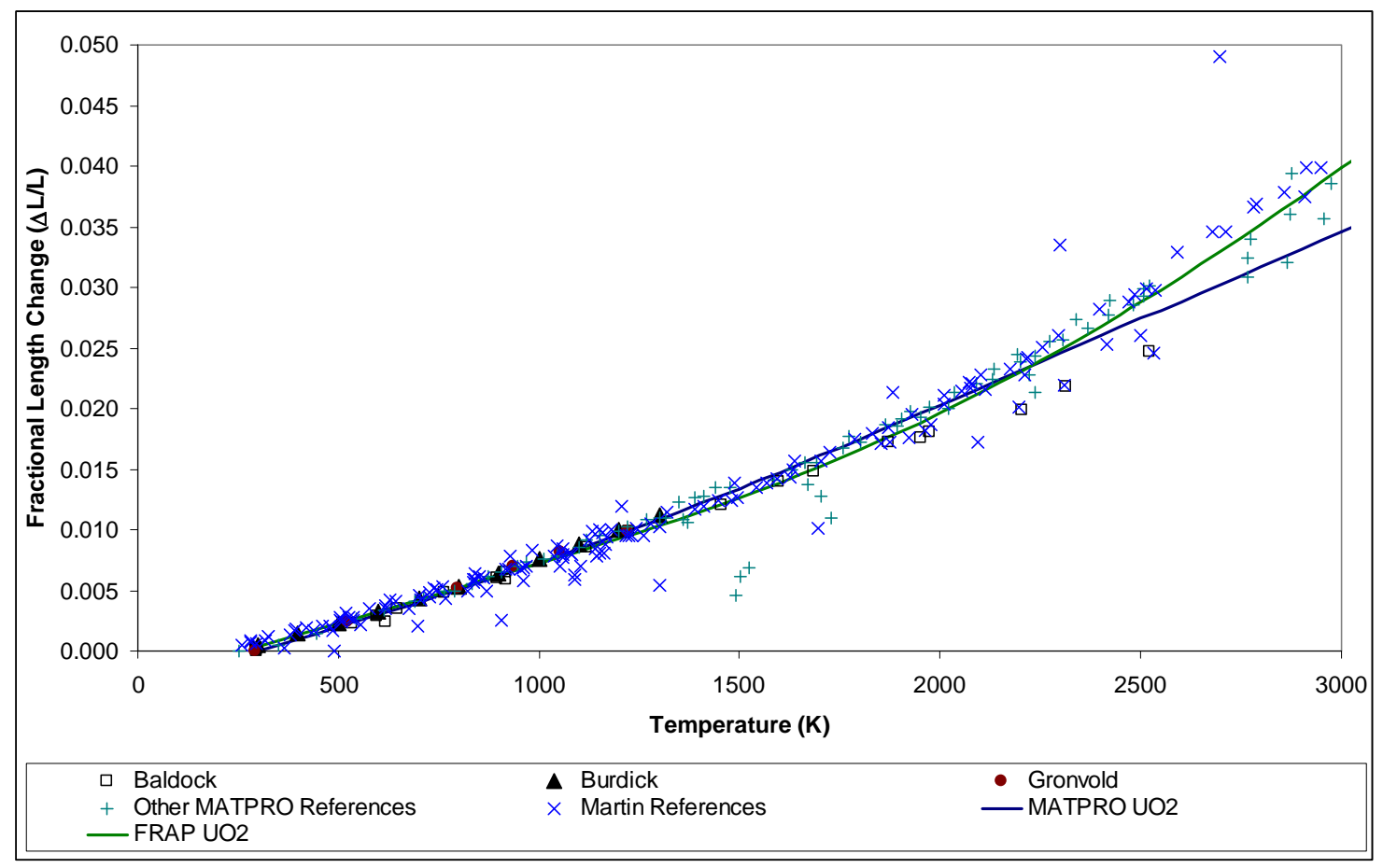

Figure 2.5-1 Model-to-data comparison for $\mathrm{UO}_{2}$ correlation used in MATPRO and FRAPCON-3/FRAPTRAN with updated constants. 


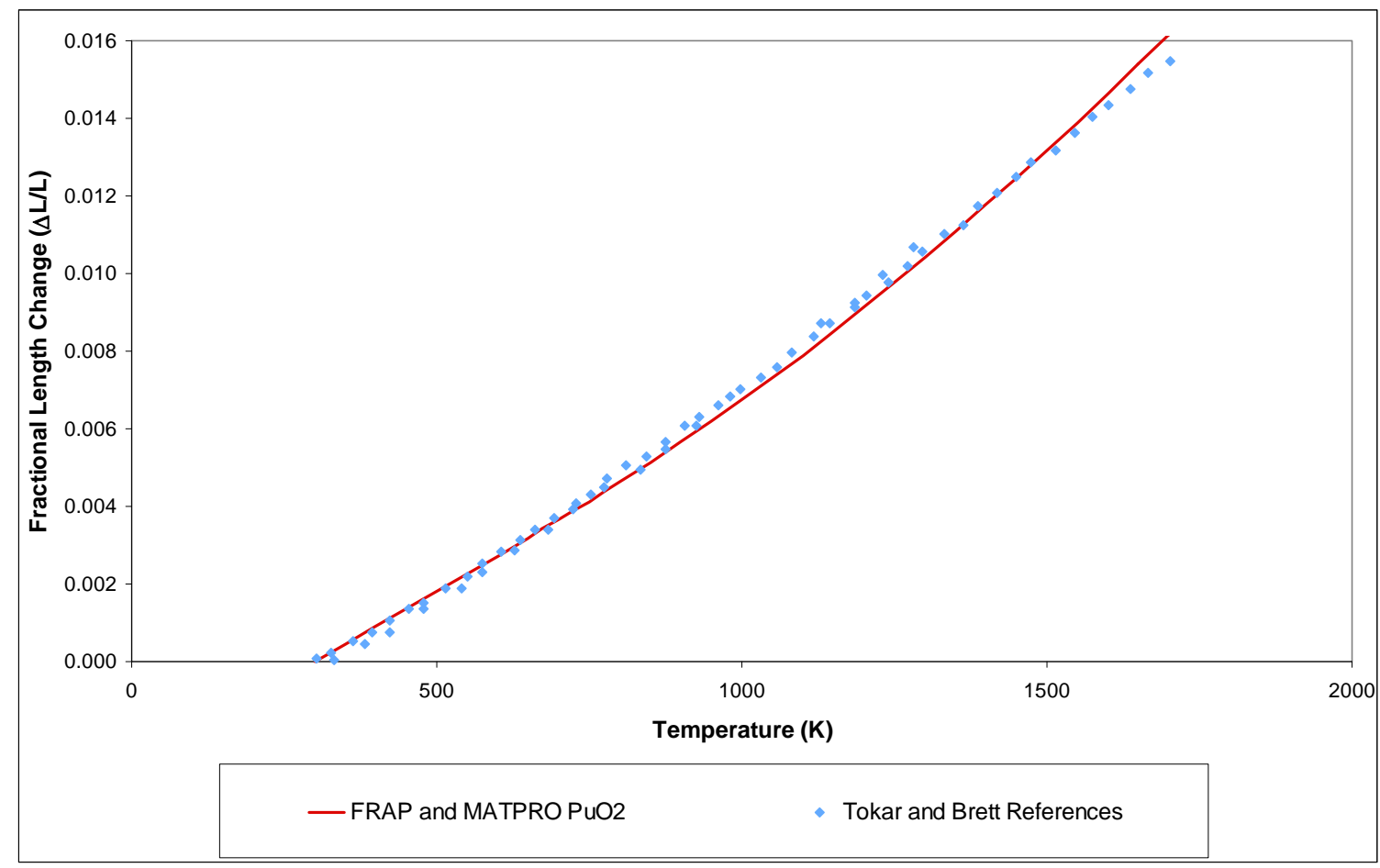

Figure 2.5-2 Model-to-data comparison for $\mathrm{PuO}_{2}$ correlation used in MATPRO and FRAPCON-3/FRAPTRAN.

\subsubsection{Applicability and Uncertainty}

The fuel thermal expansion correlation used in the FTHEXP subroutine is applicable through the melting temperature of the fuel (3111.15K for $\left.\mathrm{UO}_{2}\right)$. The uncertainty of the pooled data was found to be temperature dependent, increasing approximately linearly with temperature. Therefore, a percentage error is given rather than a fixed number. The $\pm \sigma$ limits were found to be within \pm 10 percent of the calculated value.

\subsubsection{References}

Baldock, P.J., et al. 1966. “The X-ray Thermal Expansion of Near-Stoichiometric $\mathrm{UO}_{2}$," Journal of Nuclear Materials, 18:305-313.

Burdick, M.D., and H.S. Parker. 1956. "Effect of Particle Size on Bulk Density and Strength Properties of Uranium Dioxide Specimens," Journal of the American Ceramic Society, 39:181187.

Gronvold, F. 1955. "High Temperature X-ray Study of Uranium Oxides in the $\mathrm{UO}_{2}-\mathrm{U}_{3} \mathrm{O}_{8}$ Region,” Journal of Inorganic and Nuclear Chemistry, 1:357-370.

J.A. Christensen. 1963. “Thermal Expansion and Change in Volume of Uranium Dioxide on Melting” Journal of the American Ceramic Society, 46:607-608.

Kempter, C.P., and R.E. Elliott. 1958. "Thermal Expansion of $\mathrm{UN}, \mathrm{UO}_{2}, \mathrm{UO}_{2}-\mathrm{ThO}_{2}$, and $\mathrm{ThO}_{2}$," Journal of Chemical Physics, 30:1524-1526. 
Martin, D.G. 1988. "The Thermal Expansion of Solid $\mathrm{UO}_{2}$ and (U,Pu) Mixed Oxides - A Review and Recommendations,” Journal of Nuclear Materials, 152:94-101.

Momin, A.C., E.B. Mirza, and M.D. Mathews. 1991. "High Temperature X-Ray Diffacractometric Studies on the Lattice Thermal Expansion Behavior of $\mathrm{UO}_{2}, \mathrm{ThO}_{2}$, and $\left(\mathrm{U}_{0.2} \mathrm{Th}_{0.8}\right) \mathrm{O}_{2}$ Doped with Fission Products,” Journal of Nuclear Materials, 185:308-310.

Tokar, M., and A.W. Nutt. 1972. “Thermal Expansion of $\mathrm{PuO}_{2}$ from 25 to $1420^{\circ} \mathrm{C}$,” Transactions of the American Nuclear Society, 10:210-211.

Brett, N.H., and L.E. Russel. 1960. "The Thermal Expansion of $\mathrm{PuO}_{2}$ and Some Other Actinide Oxides Between Room Temperature an $1000^{\circ} \mathrm{C}$," in Proceedings of the Second International Conference on Plutonium Metallurgy, Grenoble, France, April 19-22, 1960, pp. 397-410.

\subsection{Fuel Densification (FUDENS)}

The subroutine FUDENS calculates fuel dimensional changes due to densification of $\mathrm{UO}_{2}$ and MOX during the first few thousand hours of water reactor operation. If available, data from resintering tests should be input by the user to determine densification (Regulatory Guide 1.126 [NRC 1978]). Otherwise, the subcode FUDENS calculates densification as a function of fuel burnup, temperature, and initial density.

The data used to develop FUDENS were taken from irradiated fuel which also included swelling (Rolstad, 1974). If fuel densification is much greater than swelling during the first 1000 hours of irradiation, then, to a first approximation, swelling can be neglected during this period. This was done during the development of the FUDENS model.

The FUDENS subroutine is used in FRAPCON-3.4 and is similar to the correlation described by MATPRO. However, FUDENS is not included in FRAPTRAN because FRAPTRAN is intended for transient events occurring over a short time scale. Since densification occurs over longer time scales, it is not included in FRAPTRAN.

\subsubsection{Model Development and Comparisons}

The subroutine FUDENS uses one of two methods to calculate the maximum density change during irradiation. The RSNTR method uses the density change observed during resintering tests (1973K for 24 hours based on Regulatory Guide 1.126 [NRC 1978]) in a laboratory furnace and is the preferred input for the calculation. When RSNTR $>0$, MATPRO uses either Equation 2.6-1 or 2.6-2 and 2.6-6 while FRAPCON-3.4 uses Equations 2.6-3 and 2.6-6. If a resintering density change is not input (i.e., RSNTR $=0$ ), the TSINT method uses the initial unirradiated density of the fuel and the fuel fabrication sintering temperature and burnup for density calculations. For TSNTR calculations, both MATPRO and FRAPCON-3.4 use either Equation 2.6-4 or 2.6-5 and 2.6-6. Both the RSNTR and TSNTR methods for MATPRO and FRAPCON-3.4 are described by the following equations to calculate the maximum densification length change during irradiation.

In the MATPRO version of FUDENS, if a nonzero value for the resintering density change is input, then either Equation 2.6-1 or Equation 2.6-2 is used, depending on the fuel temperature, FTEMP (Siefken et al., 2001). 


$$
\begin{aligned}
& \text { For FTEMP }<1000 \mathrm{~K} \quad\left(\frac{\Delta \mathrm{L}}{\mathrm{L}}\right)_{\mathrm{m}}=-(0.0015) \mathrm{RSNTR} \\
& \text { For FTEMP } \geq 1000 \mathrm{~K} \quad\left(\frac{\Delta \mathrm{L}}{\mathrm{L}}\right)_{\mathrm{m}}=-(0.00285) \mathrm{RSNTR}
\end{aligned}
$$

In FRAPCON-3.4, if the resintering density is a nonzero input, then the following equation is used to calculate the maximum dimensional change.

$$
\left(\frac{\Delta \mathrm{L}}{\mathrm{L}}\right)_{\mathrm{m}}=100 * \mathrm{RSNTR} /(3.0 * \text { FDENS })
$$

If zero is input for the resintering density change, then the MATPRO and FRAPCON-3.4 models use either Equation 2.6-4 or Equation 2.6-5, depending on the fuel temperature, FTEMP (Rolstad et al., 1974).

$$
\begin{aligned}
& \text { For FTEMP }<1000 \mathrm{~K}\left(\frac{\Delta \mathrm{L}}{\mathrm{L}}\right)_{\mathrm{m}}=\frac{-22.2(100-\mathrm{DENS})}{(\mathrm{TSINT}-1453)} \\
& \text { For FTEMP } \geq 1000 \mathrm{~K} \quad\left(\frac{\Delta \mathrm{L}}{\mathrm{L}}\right)_{\mathrm{m}}=\frac{-66.6(100-\mathrm{DENS})}{(\mathrm{TSINT}-1453)}
\end{aligned}
$$

where

$$
\begin{array}{lll}
\left(\frac{\Delta \mathrm{L}}{\mathrm{L}}\right)_{\mathrm{m}} & = & \text { maximum possible dimension change of fuel due to irradiation (percent) } \\
\text { RSNTR } & = & \text { resintered fuel density change }\left(\mathrm{kg} / \mathrm{m}^{3}\right) \\
\text { FTEMP } & = & \text { fuel temperature }(\mathrm{K}) \\
\text { DENS } & = & \text { initial density (percent theoretical) } \\
\text { TSINT } & = & \text { sintering temperature }(\mathrm{K}) \\
\text { FDENS } & = & \text { starting density }\left(\mathrm{kg} / \mathrm{m}^{3}\right)
\end{array}
$$

Densification as a function of burnup is calculated using

$$
\frac{\Delta \mathrm{L}}{\mathrm{L}}=\left(\frac{\Delta \mathrm{L}}{\mathrm{L}}\right)_{\mathrm{m}}+\mathrm{e}^{[-3(\mathrm{FBU}+\mathrm{B})]}+\left(2.0 \mathrm{e}^{[-35(\mathrm{FBU}+\mathrm{B})]}\right)
$$

where

$$
\begin{array}{lll}
\left(\frac{\Delta \mathrm{L}}{\mathrm{L}}\right) & = & \text { dimension change (percent) } \\
\text { FBU } & = & \text { fuel burnup (MWd/kgU) }
\end{array}
$$



$=0$ when $\mathrm{FBU}=0$

The FUDENS subcode presented in Equation 2.6-6 is used to calculate total densification, and then the densification from the previous time step is subtracted to obtain the incremental densification. The incremental densification for the time step being considered is the output of the subcode FUDENS and is determined by Newton's method (Hamming, 1971).

The relationship between densification and burnup in Equation 2.6-6 was originally suggested by Rolstad et al. (1974). This relationship was adopted for use in the FUDENS subroutine because it successfully describes the burnup dependence of both the original data (Rolstad et al., 1974) and EPRI data (Freshley et al., 1976) that was collected shortly thereafter. Since these initial studies, there has been additional in-reactor densification data collected for $\mathrm{UO}_{2}$ and MOX fuels irradiated in thermal reactors.

Banks (1974) reported densification data on solid and annular $\mathrm{UO}_{2}$ pellets irradiated in the Winfrith Steam Generating Heavy Water Reactor and the Halden Boiling Water Reactor. The solid pellets experienced centerline temperatures of 733 to $2443 \mathrm{~K}$ over the burnup range 0.37 to 23.7 MWd/kgU. They concluded that pellets with centerline temperatures less than $1423 \mathrm{~K}$ densified during irradiation, but at higher temperatures dimensional change was affected by intergranular fission gas swelling. Therefore, only data for temperatures less than $1423 \mathrm{~K}$ were included in comparison with the FUDENS model (Equations 2.6-4, 2.6-5, and 2.6-6). The mechanism for densification was determined to be the loss of pores with initial diameters less than $2 \mu \mathrm{m}$.

Freshley et al. (1978) reported densification data on MOX pellets irradiated in the radially adjustable facility tubes of the General Electric Test Reactor. Two pins were irradiated with fuel centerline temperatures up to $2048 \mathrm{~K}$ and burnup to $4.6 \mathrm{MWd} / \mathrm{kgM}$. The pellets were fabricated with sintering temperatures from 1748 to $2008 \mathrm{~K}$ and had starting density values ranging from 91 to 95 percent TD. They concluded that dilute additions of $\mathrm{PuO}_{2}$ in $\mathrm{UO}_{2}$ (up to 6 wt $\% \mathrm{PuO}_{2}$ ) do not change the densification behavior of the fuel. The mechanism of densification was observed to be sintering of pores with initial diameters of $0.5 \mu \mathrm{m}$ or smaller.

Small (1987) irradiated four types of $\mathrm{UO}_{2}$ pellets in the Harwell Materials Test Reactor. Pellet centerline temperatures ranged from 623 to $1823 \mathrm{~K}$ and burnup ranged from 0.0025 to

2.6 MWd/kgU. Three of the four types of pellets were fabricated with pores smaller than $2 \mu \mathrm{m}$ in diameter to more carefully examine the mechanisms behind densification. For reference, fuel currently fabricated has very few pores less than $2 \mu \mathrm{m}$ in diameter. Densification was determined by measuring porosity before and after irradiation. At centerline temperatures above $1273 \mathrm{~K}$, densification was caused by sintering of pores less than $1 \mu \mathrm{m}$ in diameter.

Figure 2.6-1 compares measured and predicted values of densification using the TSNTR correlation, which is used in both FRAPCON-3.4 and MATPRO and incorporates Equations 2.6-4, 2.6-5, and 2.6-6. 


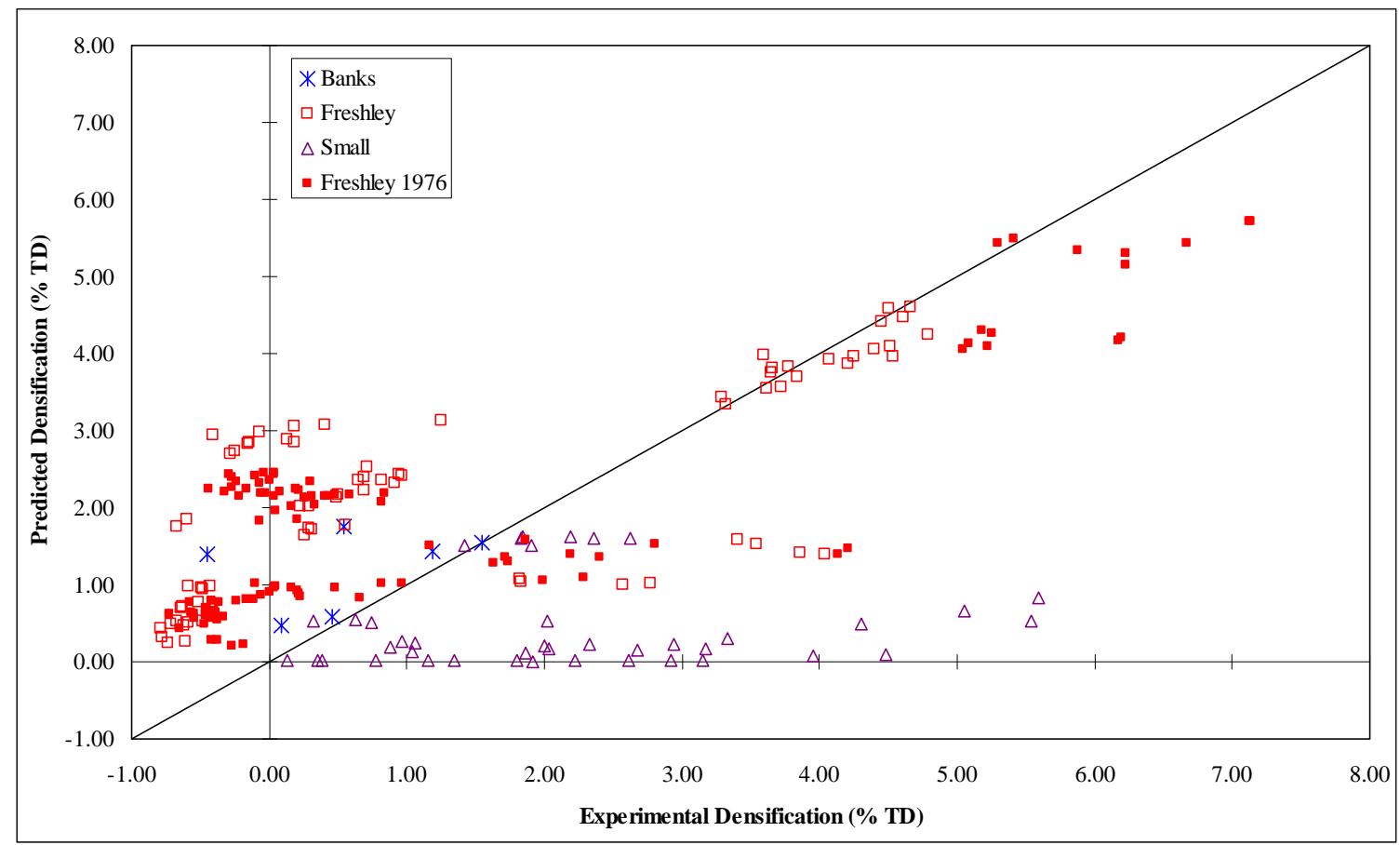

Figure 2.6-1 Comparison of densification predictions generated by the FUDENS model and experimental densification measurements determined in reactor.

This data comparison demonstrates that basing densification on the sintering temperature provides a large degree of uncertainty. However, it should be noted that the Small (1987) data, which exhibited very high densification at very low burnup (less than $0.07 \mathrm{MWd} / \mathrm{kgU}$ ), possessed a non-prototypic pore size distribution that was heavily weighted toward smaller diameters. Three of the four fuel types had 100 percent of their porosity in pores smaller than $2 \mu \mathrm{m}$ diameter, which is atypical of current fuel fabrication.

Since in-reactor densification is strongly influenced by fuel fabrication, ex-reactor re-sintering tests are the preferred method for predicting in-reactor fuel densification (NRC, 1978).

Ex-reactor resintering tests conducted by Freshly et al. (1976 and 1978) at 1600 and $1700^{\circ} \mathrm{C}$ provided reasonable estimate of in-reactor densification tests with standard deviations of 0.5 to 0.6 percent of the theoretical density.

\subsubsection{Applicability and Uncertainty}

The densification correlation used in the subroutine FUDENS is applicable to fuels with pore size distributions that are similar to those included in the Freshley et al. (1976) study. Due to the scatter in the experimental data, it is difficult to establish a meaningful measure of uncertainty.

\subsubsection{References}

Rolstad, E., et al. 1974. "Measurements of the Length Changes of $\mathrm{UO}_{2}$ Fuel Pellets During Irradiation," Enlarged HPG Meeting on Computer Control and Fuel Research, June 4-7, 1974. 
NRC - U.S. Nuclear Regulatory Commission. 1978. An Acceptable Model and Related Statistical Methods for the Analysis of Fuel Densification. Regulatory Guide 1.126, Rev.1, U.S. Nuclear Regulatory Commission, Washington, D.C.

Siefken, L.J., E.W. Coryell, E.A. Harvego, J.K. Hohorst. 2001. SCDAP/RELAP5/MOD 3.3 Code Manual MATPRO-A Library of Materials Properties for Light-Water-Reactor-Accident Analysis, NUREG/CR-6150, Vol. 4, Rev. 2, INEL-96/0422, Idaho National Engineering and Environmental Laboratory, Idaho Falls, ID.

Hamming, R.W. 1971. Introduction to Applied Numerical Analysis, New York: McGraw-Hill Book Company, Inc.

Freshley, M.D., D.W. Brite, J.L. Daniel, and P.E. Hart. 1976. "Irradiation-Induced Densification of $\mathrm{UO}_{2}$ Pellet Fuel,” Journal of Nuclear Materials, 62:138-166.

Banks, D.A. 1974. "Some Observations of Density and Porosity Changes in $\mathrm{UO}_{2}$ Fuel Irradiated in Water-Cooled Reactors,” Journal of Nuclear Materials, 54:97-107.

Freshley, M.D., D.W. Brite, J.L. Daniel, and P.E. Hart. 1978. "Irradiation-Induced Densification and $\mathrm{PuO}_{2}$ Particle Behavior in Mixed-Oxide Pellet Fuel,” Journal of Nuclear Materials, 81:6392.

Small, G.J. 1987. "Densification of Uranium Dioxide at Low Burn-Up,” Journal of Nuclear Materials, 148:302-315.

\subsection{Fuel Swelling (FSWELL)}

The subroutine FSWELL calculates fuel swelling, which is caused by the buildup of solid and gaseous fission products during irradiation. Fuel swelling (FSWELL) is combined with creep-induced elongation (FCREEP), densification due to pressure sintering (FHOTPS), and irradiation (FUDENS) to calculate the overall dimensional changes in fuel.

The gaseous swelling correlation in FSWELL is not used in FRAPCON-3 because it significantly overpredicts swelling and its effects on cladding deformation. The FSWELL correlation for solid swelling is used in both MATRPO and FRAPCON-3. However, the FSWELL solid swelling correlation used in FRAPCON-3.4 has evolved from the original model described in MATPRO. The FSWELL subroutine is not used in FRAPTRAN 1.4 because FRAPTRAN 1.4 is intended for shorter time scales than those that result in significant solid swelling.

\subsubsection{Model Development and Comparisons}

In MATPRO, the correlation used by FSWELL to calculate swelling due to the buildup of solid fission products is give by Equation 2.7.1.

$$
\mathrm{S}_{\mathrm{S}}=2.5 \times 10^{-29} \mathrm{~B}_{\mathrm{S}}
$$

where

$\mathrm{S}_{\mathrm{S}}=$ fractional volume change due to solid fission products

$\left(\mathrm{m}^{3}\right.$ volume change $/ \mathrm{m}^{3}$ fuel) 
$\mathrm{B}_{\mathrm{S}} \quad=\quad$ burnup during a time step (fissions $/ \mathrm{m}^{3}$ )

At temperatures below 2800K, swelling due to the buildup of gaseous fission products is given by Equation 2.7-2.

$$
\mathrm{S}_{\mathrm{g}}=8.8 \times 10^{-56}(2800-\mathrm{T})^{11.73} \mathrm{e}^{[-0.0162(2800-\mathrm{T})]} \mathrm{e}^{\left[-8.0 \times 10^{-27} \mathrm{~B}\right]} \mathrm{B}_{\mathrm{S}}
$$

where

$\mathrm{S}_{\mathrm{g}} \quad=\quad$ fractional volume change due to gas fission products $\left(\mathrm{m}^{3}\right.$ volume change $/ \mathrm{m}^{3}$ fuel)

$\mathrm{T}=$ temperature $(\mathrm{K})$

$\mathrm{B}=$ total burnup of fuel (fissions $/ \mathrm{m}^{3}$ )

For temperatures greater than $2800 \mathrm{~K}, \mathrm{~S}_{\mathrm{g}}$ is zero because the gas that causes swelling is assumed to have released. The correlations presented in Equations 2.7-1 and 2.7-2 are used to determine swelling due to solid and gaseous fission product buildup, respectively, in the FSWELL subcode described by MATPRO.

Unlike the MATPRO version of FSWELL, FRAPCON-3.4 does not include a correlation for swelling due to the buildup of gaseous fission products. Results of an NRC-sponsored test rod were compared with predictions made by FRAPCON-3 and revealed that swelling models that included gaseous fission product buildup tend to overpredict test results at burnup levels up to 10 GWd/MTU (Lanning and Bradley, 1984; Lanning et al., 1997). It was determined that swelling could be modeled better by eliminating the contribution from gaseous fission products and increasing the contribution of solid fission products from 0.699 percent (MATPRO and FRAPCON-2) to 0.77 percent (FRAPCON-3) per $10 \mathrm{GWd} / \mathrm{MTU}$.

In FRAPCON-3.4, the swelling due to solid fission products was decreased to 0.62 percent per $10 \mathrm{GWd} / \mathrm{MTU}$ for burnup less than $80 \mathrm{GWd} / \mathrm{MTU}$ and increased to 0.86 percent per

$10 \mathrm{GWd} / \mathrm{MTU}$ for burnup greater than $80 \mathrm{GWd} / \mathrm{MTU}$. The correlations used to calculate fuel swelling under these conditions are given in Equations 2.7-3 and 2.7-4, respectively.

For burnup $<80 \mathrm{GWd} / \mathrm{MTU}$

$$
\text { soldsw }=\text { bus } *\left(2.315 \times 10^{-23}+\text { sigswell } * 2.315 \times 10^{-24}\right)
$$

For burnup > $80 \mathrm{GWd} / \mathrm{MTU}$

$$
\text { soldsw }=\text { bus } *\left(3.211 \times 10^{-23}+\text { sigswell } * 3.211 \times 10^{-24}\right)
$$

where

$\begin{array}{lll}\text { soldsw } & = & \text { fractional volume change due to solid fission products }\left(\mathrm{m}^{3} \text { volume change } / \mathrm{m}^{3}\right. \\ \text { sigswell } & = & \text { user-defined parameter that incrementally changes error } \\ \text { bus } & = & \text { fuel burnup during time step (analogous to } \mathrm{B}_{\mathrm{S}} \text { in MATPRO model) } \\ \text { fdens } & = & \text { initial pellet density }\left(\mathrm{kg} / \mathrm{m}^{3}\right) \\ \text { bu } & = & \text { burnup at end of time step }(\mathrm{MWs} / \mathrm{kgU}) \\ \text { bul } & = & \text { burnup at end of previous time step }(\mathrm{MWs} / \mathrm{kgU})\end{array}$

and 


$$
\text { bus }=\text { fdens } * 2.974 \times 10^{10} *(\mathrm{bu}-\mathrm{bul})
$$

The variable "bus" in Equations 2.7-3 and 2.7-4 is analogous to the variable " $\mathrm{B}_{\mathrm{s}}$ " in Equation 2.7-1. However, "bus" permits the fractional volume change due to solid fission products to be determined as a function of burnup in $\mathrm{MWs} / \mathrm{kgU}$ as opposed to fissions $/ \mathrm{m}^{3}$.

A comparison between the MATPRO and the FRAPCON-3.4 versions of FSWELL is presented in Figure 2.7-1 along with experimental data from immersion density measurements. Based on this figure, it can be seen that the FRAPCON-3.4 model predicts less swelling than the MATPRO model, which tends to overpredict the experimentally determined data points.

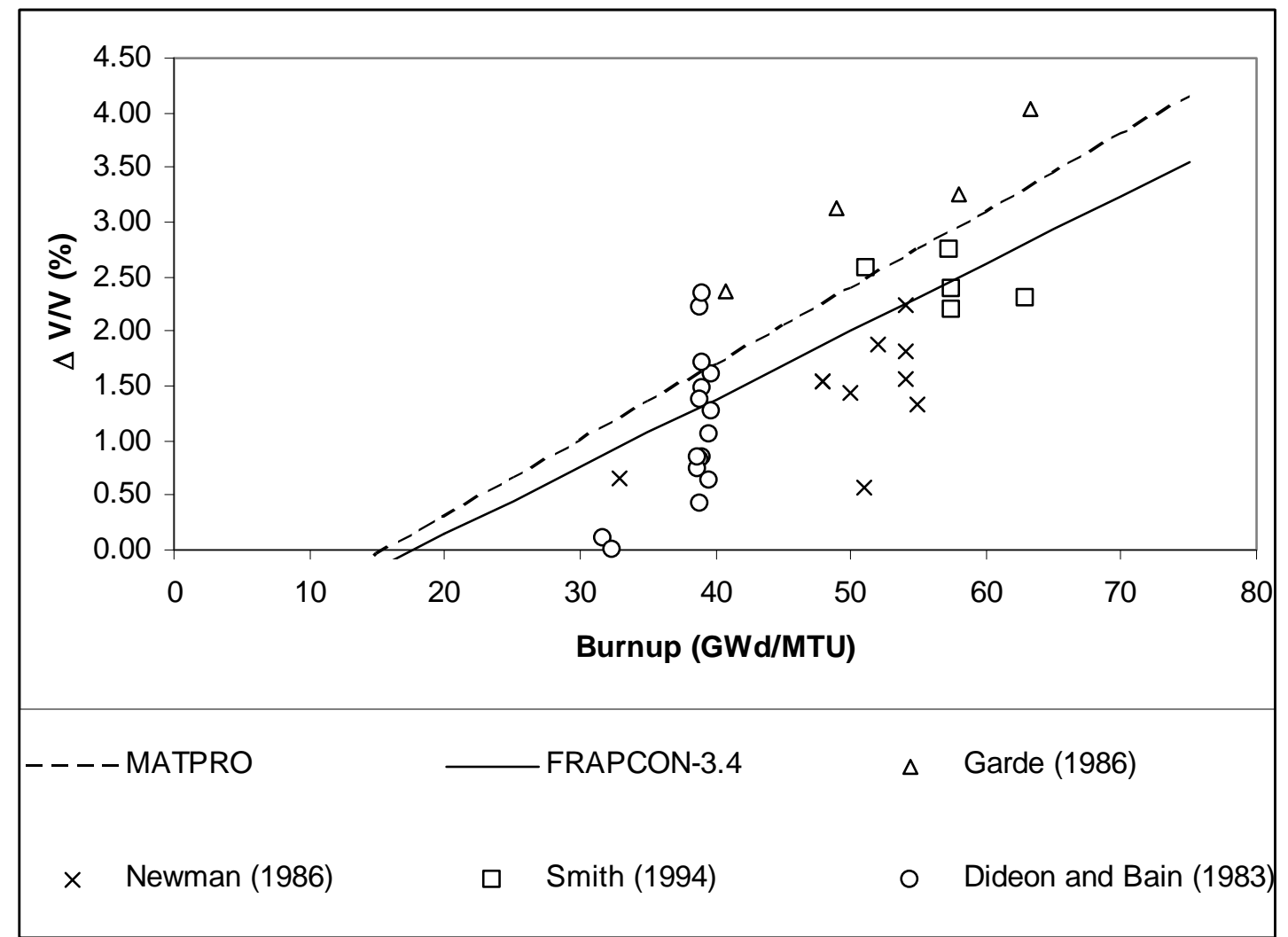

Figure 2.7-1 Data comparison between MATPRO and FRAPCON-3.4 versions of FSWELL.

For burnup $>80 \mathrm{GWd} / \mathrm{MTU}$, a higher swelling rate is used in the FRAPCON-3.4 version of FSWELL. Data comparisons between FRAPCON-3.4 predictions and data from instrumented fuel assemblies tested at Halden are presented in Figure 2.7-2 for varying levels of burnup. These Halden swelling data are based on measurements of fuel stack length and cladding diameter change with burnup and converted to volume swelling assuming isotropic swelling. The diametral cladding change data versus burnup is particularly valuable because it implicitly includes the effects of cladding restraint on fuel swelling. 


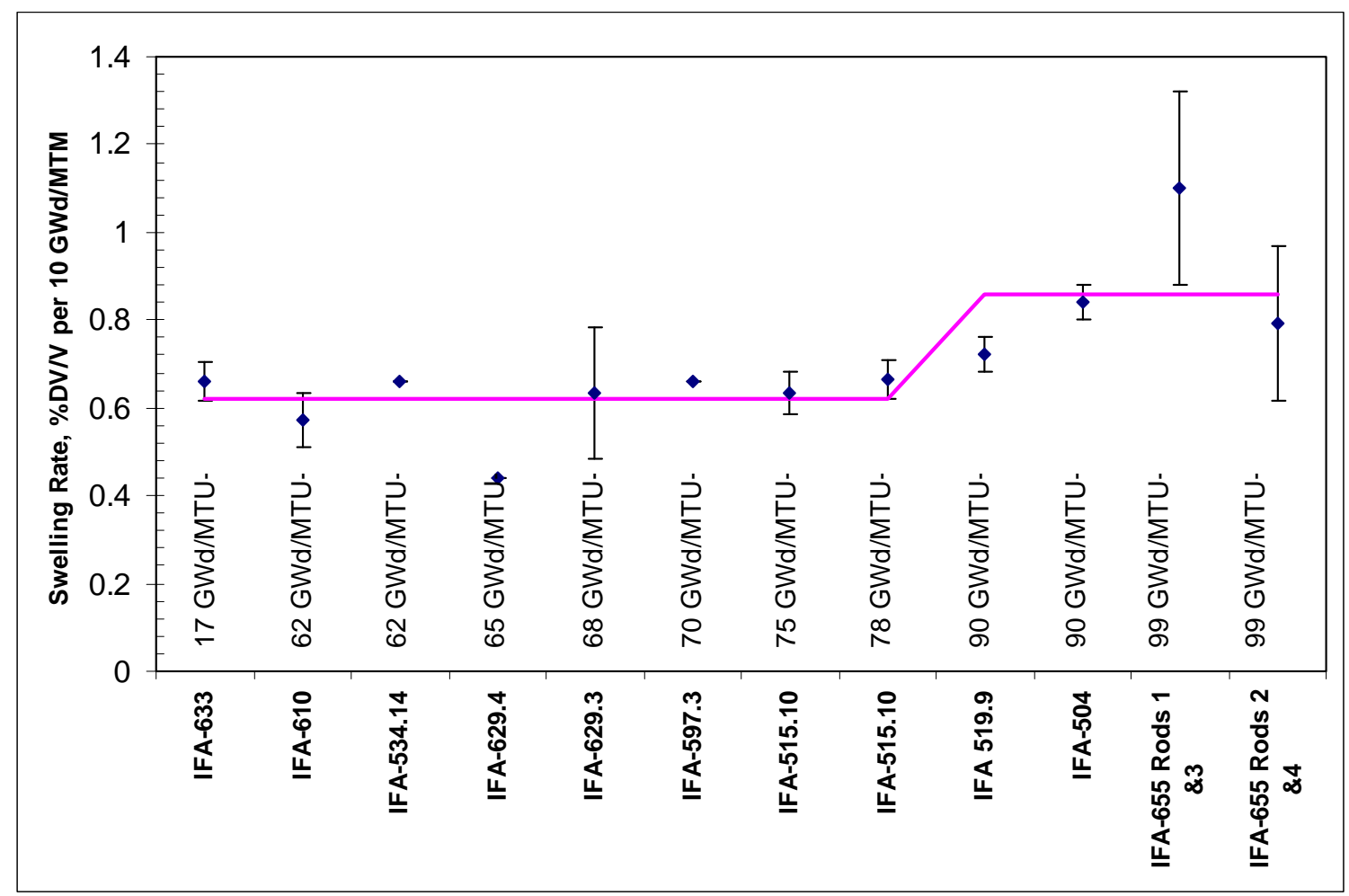

Figure 2.7-2 Comparison between swelling rates determined for various instrumented fuel assemblies tests at Halden and swelling rates predicted by FRAPCON-3.4.

\subsubsection{Applicability and Uncertainty}

Based on the data from which it is derived, the correlation used in FSWELL is applicable to fuels with burnups up to $100 \mathrm{GWd} / \mathrm{MTU}$. Below $80 \mathrm{GWd} / \mathrm{MTU}$, a 1-sigma uncertainty of 0.08 percent $\Delta \mathrm{V} / \mathrm{V}$ per $10 \mathrm{GWd} / \mathrm{MTU}$ has been calculated. Above $80 \mathrm{GWd} / \mathrm{MTU}$, a 1 sigma uncertainty of 0.16 percent $\Delta \mathrm{V} / \mathrm{V}$ per $10 \mathrm{GWd} / \mathrm{MTU}$ has been calculated.

\subsubsection{References}

Lanning, D.D., and E.R. Bradley. 1984. Irradiation History and Interim Postirradiation Data for IFA-432, NUREG/CR-3071, Pacific Northwest Laboratory for the U.S. Nuclear Regulatory Commission, Washington, D.C.

Lanning, D.D. C.E. Beyer, C.L. Painter. 1997. FRAPCON-3: Modifications to Fuel Rod Material Properties and Performance Models for High-Burnup Application, NUREG/CR-6534, Vol. 1, Pacific Northwest National Laboratory for the U.S. Nuclear Regulatory Commission, Washington, D.C.

Garde, A.M. 1986. Hot Cell Examination of Extended Burnup Fuel from Fort Calhoun, DOE/ET-34030-11 (CEND-427), Combustion Engineering, Windsor, CT.

Newman, L.W. 1986. The Hot Cell Examination of Oconee 1 Fuel Rods after Five Cycles of Irradiation, DOE/ET-34212-36, Babcock and Wilcox, Lynchburg, VA. 
Smith, G.P., R.C. Pirek, H.R. Freeburn, and D. Schrire. 1994. The Evaluation and Demonstration of Methods for Improved Nuclear Fuel Utilization, DOE/ET-34-013-15, Combustion Engineering, Windsor, CT.

Dideon, C.G., and G.M. Bain. 1983. Fuel Performance under Extended Burnup for the B\&W 15x15 Design, DOE/ET-34212-38, Babcock and Wilcox, Lynchburg, VA. 


\subsection{Cladding Material Properties}

Material property correlations for fuel claddings are described in the following subsections. Unless otherwise specified, the correlations below are applicable to Zircaloy-2, -4, ZIRLO, and M5. In addition, several FRAPTRAN 1.4 subroutines include additional correlations to describe a Russian zirconium alloy containing $1 \mathrm{wt} \%$ niobium (ZrNb-1). The subroutine used to determine the material correlation in either the FRAPCON-3 or FRAPTRAN codes is given in parentheses.

\subsection{Cladding Specific Heat (CCP)}

The specific heat subcode, CCP, determines the true specific heat at constant pressures for cladding. Specific heat calculations are based on interpolation of measured data. The correlation in FRAPCON-3.4, FRAPTRAN 1.4, and MATPRO is applicable to Zircaloy-2, Zircaloy-4, ZIRLO, and M5 alloys. However, FRAPTRAN 1.4 contains correlations to calculate the specific heat of ZrNb-1. This calculation can be based on data collected at either fast or slow heating rates and the user can designate the appropriate data set.

\subsubsection{Model Development and Comparisons}

The CCP subcode requires temperature as an input to calculate specific heat. For the alpha phase of the Zircaloy alloys (temperature less than 1090K), CCP returns linear interpolations for the points listed in Table 3.1-1. These data points are based on precise data taken by Brooks and Stansbury (1966) with a Zircaloy-2 sample that had been vacuum-annealed at $1075 \mathrm{~K}$ to remove hydrogen, which would have otherwise affected the measurement.

The standard errors associated with this interpolation technique differ between MATPRO and FRAPCON-3.4/FRAPTRAN 1.4. In MATPRO, the standard error of the CCP interpolation was based on 90 points in the Brooks and Stansbury (1966) database and was found to be temperature dependent. For the 57 data points between 300 and $800 \mathrm{~K}$, the standard error is $1.1 \mathrm{~J} / \mathrm{kg}^{*} \mathrm{~K}$. Between 800 and $1090 \mathrm{~K}$, it is $2.8 \mathrm{~J} / \mathrm{kg}^{*} \mathrm{~K}$. The FRAPCON-3.4/FRAPTRAN 1.4 codes assume a standard error of $10 \mathrm{~J} / \mathrm{kg} * \mathrm{~K}$ below $1090 \mathrm{~K}$.

For temperatures from 1090 to 1300K (where Brooks and Stansbury do not report results), values of specific heat proposed by Deem and Eldridge (1967) are adopted. The Deem and Eldridge (1967) values are based on measurements of enthalpy and temperature which provide considerably less precise specific heat data than the results of Brooks and Stansbury (1966).

As a result, the MATPRO standard error estimated from the Deem and Eldridge (1967) data in the region of 1090 through $1310 \mathrm{~K}$ is $10.7 \mathrm{~J} / \mathrm{kg} * \mathrm{~K}$. This standard error is a measure only of the precision of the fit, since only a single data source is used. The standard error in FRAPCON-3.4/FRAPTRAN is assumed to be $25 \mathrm{~J} / \mathrm{kg}^{*} \mathrm{~K}$ between 1090 and $1300 \mathrm{~K}$.

Above the alpha + beta to beta transformation temperature (about 1250K) and up to about $1320 \mathrm{~K}$, a constant value of $355.7 \mathrm{~J} / \mathrm{kg} * \mathrm{~K}$ was reported by Deem and Eldridge (1967). This value agrees well with a value of 365.3 reported by Coughlin and King (1950) for pure beta zirconium. The standard error of specific heat calculations made above $1300 \mathrm{~K}$ is assumed to be $100 \mathrm{~J} / \mathrm{kg}^{*} \mathrm{~K}$ in the FRAPCON-3.4/FRAPTRAN 1.4 codes. 
In addition to Zircaloy alloys, FRAPTRAN 1.4 has been modified to include specific heat calculations for ZrNb-1. These calculations are based on data collected at two different heating rates. Depending on the user input, specific heat calculations can be based on either the fast or the slow heating rate data. The data used to interpolate specific heat values for the ZrNb-1 alloy are presented in Table 3.1-2. There is no standard error described for specific heat calculations based on these data sets.

Table 3.1-1 Specific heat capacity database for Zircaloy-2, Zircaloy-4, ZIRLO, and M5.

\begin{tabular}{|c|c|c|c|c|}
\hline \multirow[b]{2}{*}{$\frac{\text { Temperature }}{(\mathrm{K})}$} & \multirow[b]{2}{*}{$\frac{\text { Specific Heat }}{\underline{\text { Capacity }}}$} & \multirow[b]{2}{*}{ Source } & \multicolumn{2}{|c|}{ Standard Error } \\
\hline & & & $\frac{\text { MATPRO }}{\left(\mathrm{J} / \mathrm{kg}^{*} \mathrm{~K}\right)}$ & $\frac{\frac{\text { FRAPCON-3 / }}{\text { FRAPTRAN }}}{\frac{\left(\mathrm{J} / \mathrm{kg}^{*} \mathrm{~K}\right)}{2}}$ \\
\hline 300 & 281 & Brooks and Stansbury & 1.1 & 10 \\
\hline 400 & 302 & Brooks and Stansbury & 1.1 & 10 \\
\hline 640 & 331 & Brooks and Stansbury & 1.1 & 10 \\
\hline 1090 & 375 & Brooks and Stansbury & 2.8 & 10 \\
\hline 1093 & 502 & Deem and Eldridge & 10.7 & 25 \\
\hline 1113 & 590 & Deem and Eldridge & 10.7 & 25 \\
\hline 1133 & 615 & Deem and Eldridge & 10.7 & 25 \\
\hline 1153 & 719 & Deem and Eldridge & 10.7 & 25 \\
\hline 1173 & 816 & Deem and Eldridge & 10.7 & 25 \\
\hline 1193 & 770 & Deem and Eldridge & 10.7 & 25 \\
\hline 1213 & 619 & Deem and Eldridge & 10.7 & 25 \\
\hline 1233 & 469 & Deem and Eldridge & 10.7 & 25 \\
\hline 1248 & 356 & Deem and Eldridge & 10.7 & 25 \\
\hline 2098 & 356 & Coughlin and King & 100 & 100 \\
\hline 2099 & 356 & Coughlin and King & 100 & 100 \\
\hline
\end{tabular}

Table 3.1-2 Specific heat capacity database for ZrNb-1 alloys.

$\begin{array}{ccl}\frac{\text { Temperature }}{(\mathrm{K})} & \frac{\text { Specific Heat Capacity }}{(\mathrm{J} / \mathrm{kg} \mathrm{K})} & \underline{\text { Source }} \\ & & \\ & & \\ & & \\ 280 & 345 & \\ 473 & 360 & \text { Volkov B. Yu et al. } \\ 573 & 370 & \text { Volkov B. Yu et al. } \\ 673 & 380 & \text { Volkov B. Yu et al. } \\ 773 & 383 & \text { Volkov B. Yu et al. } \\ 873 & 385 & \text { Volkov B. Yu et al. } \\ 883 & 448 & \text { Volkov B. Yu et al. } \\ 973 & 680 & \text { Volkov B. Yu et al. } \\ 1025 & 816 & \text { Volkov B. Yu et al. } \\ 1073 & 770 & \text { Volkov B. Yu et al. } \\ & & \text { Volkov B. Yu et al. }\end{array}$




\begin{tabular}{ccc}
\hline 1153 & 400 & Volkov B. Yu et al. \\
1173 & 392 & Volkov B. Yu et al. \\
1248 & 356 & Volkov B. Yu et al. \\
& -------- High Heating Rate $(>$ 1000 K/s) -------- \\
1100 & 412 & Ljusternik V.E. et al. \\
1110 & 420 & Ljusternik V.E. et al. \\
1120 & 480 & Ljusternik V.E. et al. \\
1134 & 600 & Ljusternik V.E. et al. \\
1142 & 1000 & Ljusternik V.E. et al. \\
1150 & 1400 & Ljusternik V.E. et al. \\
1155 & 1600 & Ljusternik V.E. et al. \\
1161 & 1400 & Ljusternik V.E. et al. \\
& & \\
\hline
\end{tabular}

\subsubsection{Applicability and Uncertainty}

The CCP subroutine is valid over the temperature range from which the data sets have been collected on zirconium alloys (300 to 2099K). This correlation is applied to Zircaloy-2, -4, ZIRLO, and M5. However the standard error increases with increasing temperature as indicated in Table 3.1-1.

\subsubsection{References}

Brooks, C.R., and E.E. Stansbury. 1966. “The Specific Heat of Zircaloy-2 from 50 to $700^{\circ}$ C,” Journal of Nuclear Materials, 18:223.

Deem, H.W., and E.A. Eldridge. 1967. Specific Heats of Transformation of Zirclaoy-2 and Low Nickel Zircaloy-2, USAEC BM1-1803, Battelle Memorial Institute, Columbus, $\mathrm{OH}$.

Coughlin, J.P., and E.G. King. 1950. "High-Temperature Heat Contents of Some ZirconiumContaining Substances,” Journal for the American Chemical Society, 72:2262.

Volkov, B. Yu., et al. 1989. Library of Subprograms on Physical and Mechanical Properties of the N1-Alloy Fuel Rod Cladding Material, Preprint IAE-4941/11, Institute of Automation and Electrometry, Moscow, Russia.

Ljusternik, V.E., et al. 1993. "Experimental research of zirconium reactor materials thermal properties: 110-alloy,” M.J. Higher Temperature Thermal Physics, 31(4).

\subsection{Cladding Thermal Conductivity (CTHCON)}

The subroutine CTHCON is used to calculate cladding thermal conductivity, which is required for accurate predictions of fuel temperature. The thermal conductivity of the cladding is primarily a function of temperature. Other characteristics, such as residual stress levels, crystal orientation, and minor composition differences, may have secondary effects on thermal conductivity. The correlation used in CTCHCON to calculate cladding thermal conductivity is the same in MATPRO, FRAPCON-3.4, and FRAPTRAN 1.4. This correlation is applied to Zircaloy-2, -4, ZIRLO, and M5. In addition, FRAPTRAN 1.4 includes a correlation to calculate the cladding thermal conductivity of ZrNb-1. 


\subsubsection{Model Development and Comparisons}

Considering only temperature as the defining parameter, the thermal conductivity of Zircaloy for temperatures less than 2098K is described by CTHCON in Equation 3.2-1. The uncertainty of the conductivity calculations is given in Equation 3.2-2.

$$
\begin{gathered}
\mathrm{k}=7.51+2.09 \times 10^{-2} \mathrm{~T}-1.45 \times 10^{-5} \mathrm{~T}^{2}+7.67 \times 10^{-9} \mathrm{~T}^{3} \\
\sigma_{\mathrm{k}}=1.01
\end{gathered}
$$

For temperatures greater than or equal to $2098 \mathrm{~K}$, the thermal conductivity and uncertainty are given in Equations 3.2-3 and 3.2-4, respectively.

$$
\begin{aligned}
& k=36 \\
& \sigma_{k}=5
\end{aligned}
$$

where

$\begin{array}{lll}\mathrm{k} & = & \text { thermal conductivity of Zircaloy }\left(\mathrm{W} / \mathrm{m}^{*} \mathrm{~K}\right) \\ \mathrm{T} & = & \text { temperature }(\mathrm{K}) \\ \sigma_{\mathrm{k}} & = & \text { standard deviation }(\mathrm{W} / \mathrm{m} * \mathrm{~K})\end{array}$

Equation 3.2-1 predicts k very well from room temperature to the data limit of about $1800 \mathrm{~K}$ (Figure 3.2-1) and may be extrapolated with some confidence to the melting point. The standard deviation $\left(\sigma_{\mathrm{k}}\right)$ of the data with respect to this correlation appears to be temperature independent over the data range.

The standard deviation of the constants in Equation 3.2-1 is 20 to 30 percent of the value of the constant. Jensen (1969) performed a parametric analysis of several variables involved in the estimation of fuel and cladding temperatures. Both steady-state and transient analysis showed that variations of \pm 20 percent resulted in calculated cladding temperature variations of about 2.8K. Fuel centerline temperatures are more sensitive to cladding thermal conductivity and showed variations of 28K. Similar findings were reported by Korber and Unger (1974).

For temperatures less than $2133 \mathrm{~K}$, the thermal conductivity of the ZrNb-1 alloy is calculated by FRAPTRAN 1.4 with the correlation presented in Equation 3.2-5.

$$
\mathrm{k}=15.0636 \mathrm{e}^{0.000461843 * \mathrm{~T}}
$$

For temperatures above $2133 \mathrm{~K}$, the thermal conductivity of the $\mathrm{ZrNb}-1$ cladding is assumed to be $36 \mathrm{~W} / \mathrm{m} * \mathrm{~K}$.

Figure 3.2-1 compares the CTHCON model as incorporated in MATPRO, FRAPCON-3.4, and FRAPTRAN-1.4 with the data on which it is based, extrapolated to the melting point of Zircaloy (2098K).

Figure 3.2-2 compares the CTHCON model with the Zircaloy thermal conductivity and 
diffusivity data that were not included in development of the model. Thermal diffusivity data were converted to thermal conductivity using the recommended models in Fink (2000) for specific heat and density, respectively. Although different alloys were examined, there were no discernable differences between the alloys. The scatter in the data due to differences in alloy, form, or measurement direction is comparable to the variation between individual data sets. The scatter in the data is distributed relatively uniformly around the curve representing the CTHCON model, except for anomalously high Peggs data below $1000 \mathrm{~K}$ and the anomalously low Bunnell et al. (1983) data above 1300K.

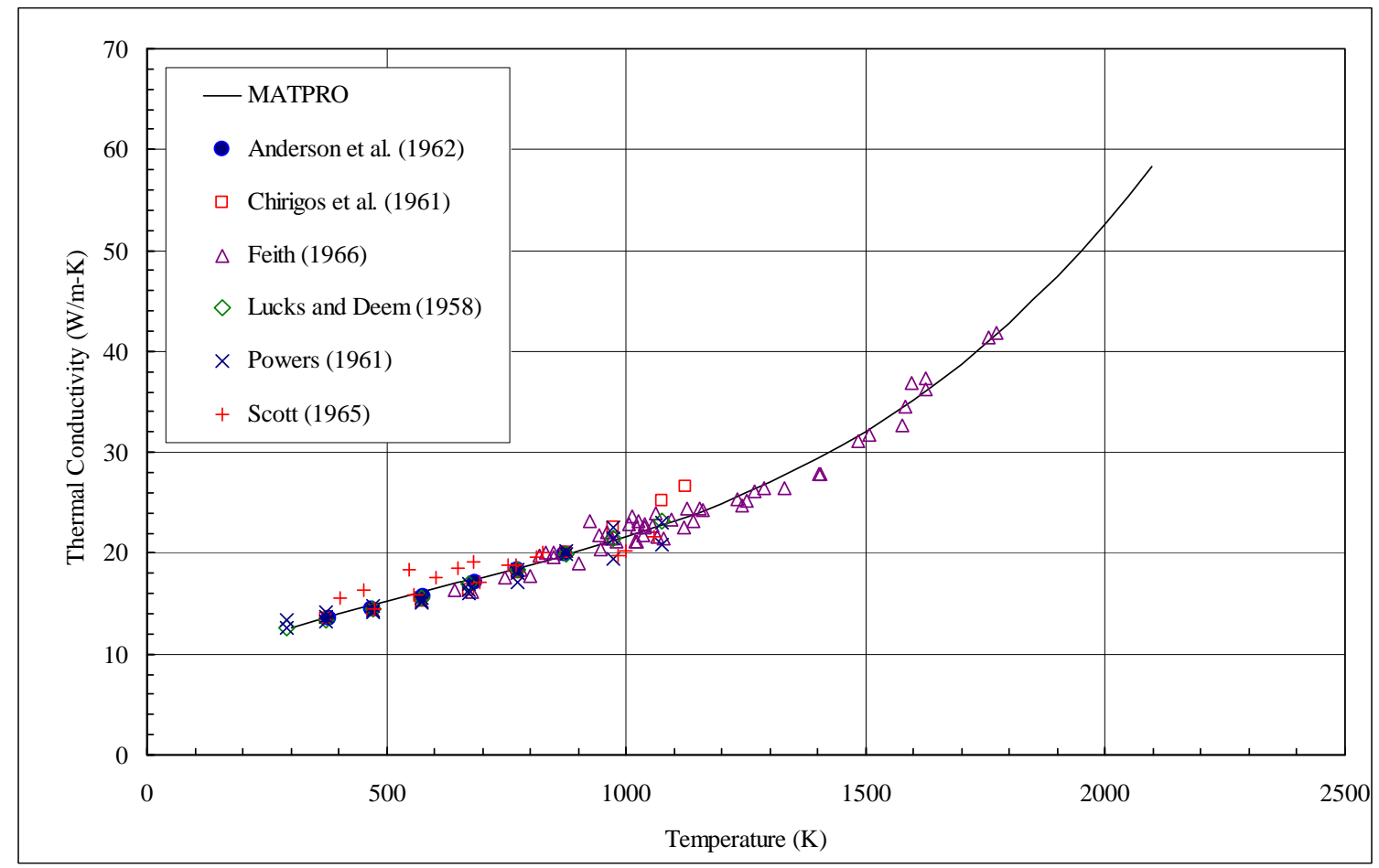

Figure 3.2-1 CTHCON model and data used for development. 


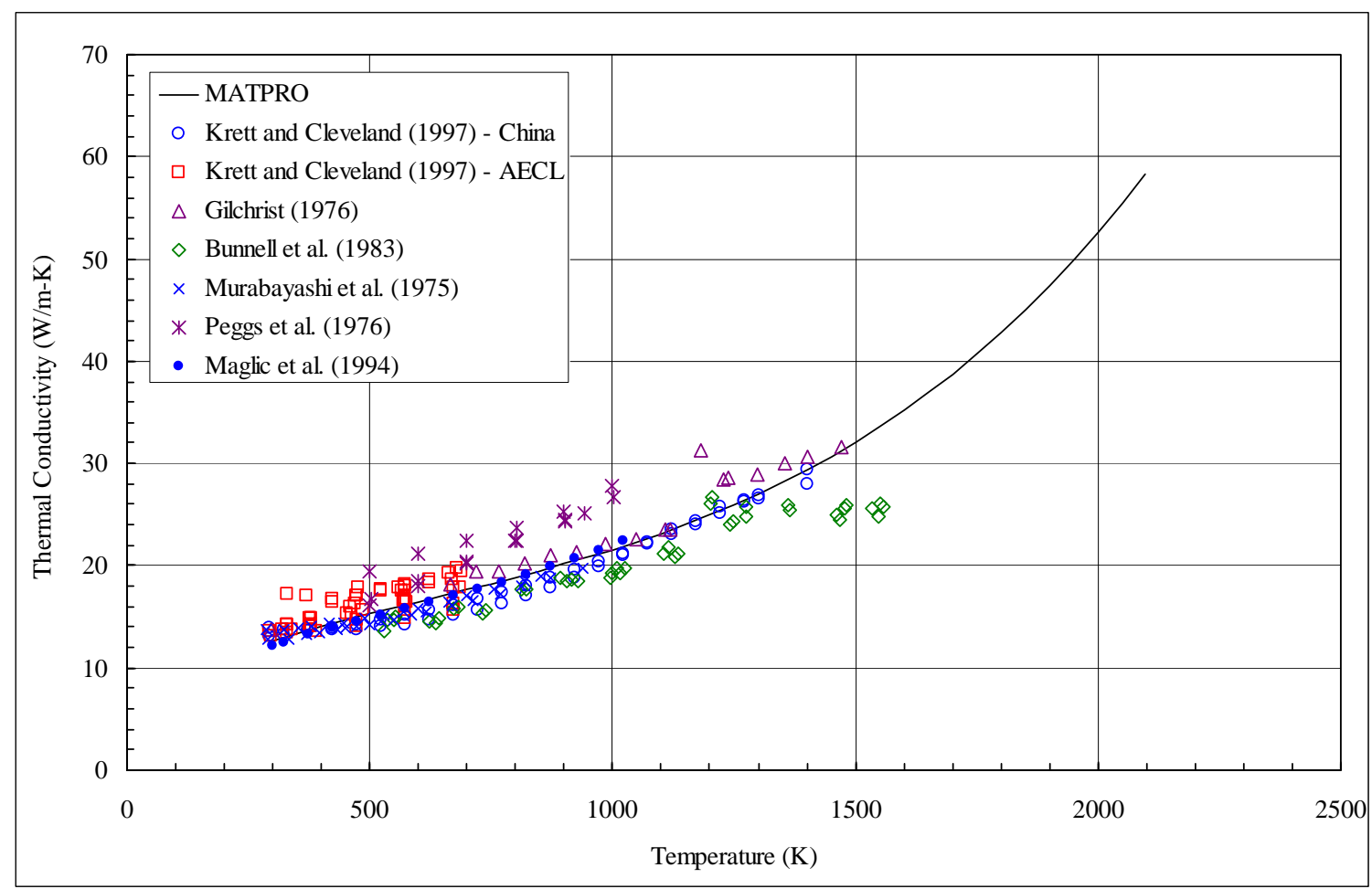

Figure 3.2-2 CTHCON model and data collected since development.

\subsubsection{Applicability and Uncertainty}

The correlation used in CTHCON to calculate the cladding thermal conductivity may be applied to temperatures up to the melting temperature of Zircaloy (2098K). The uncertainty through this range of temperature is given by Equation 3.2-2. Above the melting temperature, the uncertainty increases to the value given by Equation 3.2-4.

\subsubsection{References}

Jensen, S.E. 1969. Parametric Studies of Fuel Pin Temperature Response, IDO-17295, Philips Petroleum Company, Atomic Energy Division, Idaho Falls, ID.

Korber, H., and H.E. Unger. 1974. "Sensitivity Study on Core Heatup and Meltdown by Variation of Heat Conductivity and Thermal Emissivity," Transactions for the American Nuclear Society, 18:234-235.

Fink, J.K. 2000. “Zircaloy Thermal Conductivity,” International Nuclear Safety Center Material Properties Database, http://www.insc.anl.gov/matprop/zircaloy/index.php, Argonne National Laboratory, Argonne, IL.

Bunnell, L.R., J.L. Bates, and G.B. Mellinger. 1983. "Some High-Temperature Properties of Zircaloy-Oxygen Alloys,” Journal of Nuclear Materials, 116(2-3):219-232.

Anderson, W.K., C.J. Beck, A.R. Kephar, and J.S. Theilacker. 1962. “Zirconium Alloys,” Reactor Structural Materials: Engineering Properties as Affected by Nuclear Reactor Service, 
ASTM-STP-314, pp. 62-93, American Society for Testing and Materials, West Conshohocken, PA.

Chirigos, J.N., et al. 1961. “Development of Zircaloy-4,” Fuel Element Fabrication, New York: Academic Press, pp. 19-55.

Feith, A.D. 1966. Thermal Conductivity and Electrical Resistivity of Zircaloy-4, GEMP-669, October 1966, General Electric Company, Missile and Space Division, Cincinnati, OH.

Lucks, C.F., and H.W. Deem. 1958. Progress Relating to Civilian Applications during June, 1958, R.W. Dayton and C.R. Tipton, Jr., eds., BMI-1273, pp. 7-9, Battelle Memorial Institute, Columbus, $\mathrm{OH}$.

Powers, A.E. 1961. Application of the Ewing Equation for Calculating Thermal Conductivity from Electrical Conductivity from Electrical Conductivity, KAPL-2146, Knolls Atomic Power Laboratory, General Electric Company, Schenectady, NY.

Scott, D.B. 1965. Physical and Mechanical Properties of Zircaloy 2 and 4, WCAP-3269-41, pp. 5, 9, Westinghouse Electric Corporation, Atomic Power Division, Pittsburgh, PA.

Krett, V., and J. Cleveland. 1997. Thermophysical Properties of Materials for Water Cooled Reactors, IAEA-TECDOC-949, pp. 67-84, International Atomic Energy Agency, Vienna, Austria.

Gilchrist, K.E. 1976. "Thermal Property Measurements on Zircaloy-2 and Associated Oxide Layers up to $1200^{\circ}$ C,” Journal of Nuclear Materials, 62:257-264.

Murabayashi, M., S. Tanaka, and Y. Takahashi. 1975. "Thermal Conductivity and Heat Capacity of Zircaloy-2, -4 and Unalloyed Zirconium," Journal of Nuclear Science and Technology, 12(10):661-662.

Peggs, I.D., A.M. Stadnyk, and D.P. Godin. 1976. “Thermophysical Properties of ZirconiumAlloy Fuel Channel Components,” High Temperatures-High Pressures, 8(4):441-450.

Maglic, K.D., N.L.j. Perovic, and A.M. Stanimirovic. 1994. "Calorimetric and Transport Properties of Zircalloy 2, Zircalloy 4, and Inconel 625,” International Journal of Thermophysics, 15(4):741-755.

\subsection{Cladding Oxide Thermal Conductivity (ZOTCON)}

The ZOTCON subroutine calculates the thermal conductivity of the zirconium oxide layer that forms on zirconium alloys. Cladding temperature is the only parameter used to calculate zirconium oxide thermal conductivity. The correlation used in the ZOTCON subroutine differs between MATPRO and the FRAPCON-3.4/FRAPTRAN 1.4 codes. The MATPRO correlation is based on an arbitrary correction to an earlier correlation that was fit to a large database of oxide thermal conductivity measurements. This database contained measurements from a variety of zirconium oxide materials and test methods. The correction was applied to better fit data that was more representative of the oxide layer that forms on zirconium alloys. The correlation used in FRAPCON-3.4 and FRAPTRAN 1.4 is based on measurements obtained by Kingery (1954) from fully dense and porous (87 percent TD) zirconium oxides. 


\subsubsection{Model Development and Comparisons}

Oxide thermal conductivity models in MATPRO and FRAPCON-3.4/FRAPTRAN 1.4 are only a function of temperature. After the cladding temperature is established, the thermal conductivity of the oxide layer can be calculated using the correlation determined from either the MATPRO or the FRAPCON-3.4/FRAPTRAN 1.4 databases.

The MATPRO (2001) correlation is derived from a database that includes data collected by Adams (1954), Maki (1973), Lapshov and Bashkatov (1973), and Gilchrist (1976). The correlation used in FRAPCON-3.4/FRAPTRAN 1.4 is derived from data collected by Kingery (1954). The FRAPCON-3.4/FRAPTRAN correlation and database was adopted from an earlier version of MATPRO (Hagrman et al., 1981).

The database for the MATPRO (2001) model includes data from a variety of zirconium oxide materials and test conditions. The Maki (1973) data include two samples oxidized in steam over a small temperature range and show a sharp increase in conductivity between 400 and 500K. The principal recommendation for the data is that they were taken with black oxide from Zircaloy tubes. Two sets of data attributed to Waldman by Maki are also shown in the table. The data of Lapshov and Bashkatov (1973) are from films formed by plasma sputtering of zirconium dioxide on tungsten substrates. However, these data may not be representative of Zircaloy cladding oxide thermal conductivity because sputtered coatings are quite porous. In addition, these films do not have the same $\mathrm{O} / \mathrm{M}$ ratio as cladding oxide and may not adhere well to the substrate. The Gilchrist (1976) data includes two types of oxide films. One is a nodular oxide and the other a black oxide characteristic of the kinds of layers usually reported in high-temperature tests with cladding. The nodular oxide thermal conductivities are much lower than the black oxide thermal conductivities, and both kinds of oxide have conductivities that are significantly lower than the stabilized Zircaloy dioxide conductivities reported by Adams (1954). Considerable uncertainty is reported by Gilchrist (1976) because of difficulty in measuring oxide film thickness.

The database for the FRAPCON-3.4 and FRAPTRAN 1.4 model is based on data from Kingery (1954). The data are from two different samples; one was a bulk sample of 100 percent dense $\mathrm{ZrO}_{2}$ and the other with approximately 13 percent porosity.

Each database was fit with a different model to relate the thermal conductivity of the cladding oxide layer to temperature. The equations used to describe these models are presented in Equations 3.3-1 and 3.3-2 for MATPRO and FRAPCON-3.4/FRAPTRAN 1.4 (i.e., FRAP), respectively. These models relate the thermal conductivity of the cladding oxide layer, $\mathrm{K}$ $(\mathrm{W} / \mathrm{m} * \mathrm{~K})$, to cladding temperature, $\mathrm{T}(\mathrm{K})$.

$$
\begin{gathered}
\mathrm{K}_{\text {MATPRO }}=0.835+1.81 \times 10^{-4} \mathrm{~T} \\
\mathrm{~K}_{\mathrm{FRAP}}=1.9599-\mathrm{T}^{*}\left(2.41 \times 10^{-4}-\mathrm{T}^{*}\left(6.43 \times 10^{-7}-\mathrm{T}^{*} 1.946 \times 10^{-10}\right)\right)
\end{gathered}
$$

Both databases are presented in Figure 3.3-1 along with both cladding oxide thermal conductivity models. In addition, a more recent data set from Gilchrist (1979) is included. Figure 3.3-1 reveals that the FRAP model fits the Kingery data from which it was derived and agrees well with the measurements performed by Adams (1954). The FRAP model tends to overestimate both the Gilchrist data sets and the data from Lapshov and Bashkatov (1973). However, as stated earlier, the earlier Gilchrist (1976) data is associated with considerable uncertainty and the data from 
Lapshov and Bashkatov (1973) is likely to be low due to different O/M ratios and high porosity in the sputtered films.

The MATPRO model was initially fit to the data collected by Adams (1954), but it was adjusted to the form presented in Equation 3.3-1 to better fit the Gilchrist (1976) data for black oxide. The rationale for this correction was that the black oxide was more prototypic of the cladding oxide layer and was achieved by dividing the original equation by two. 


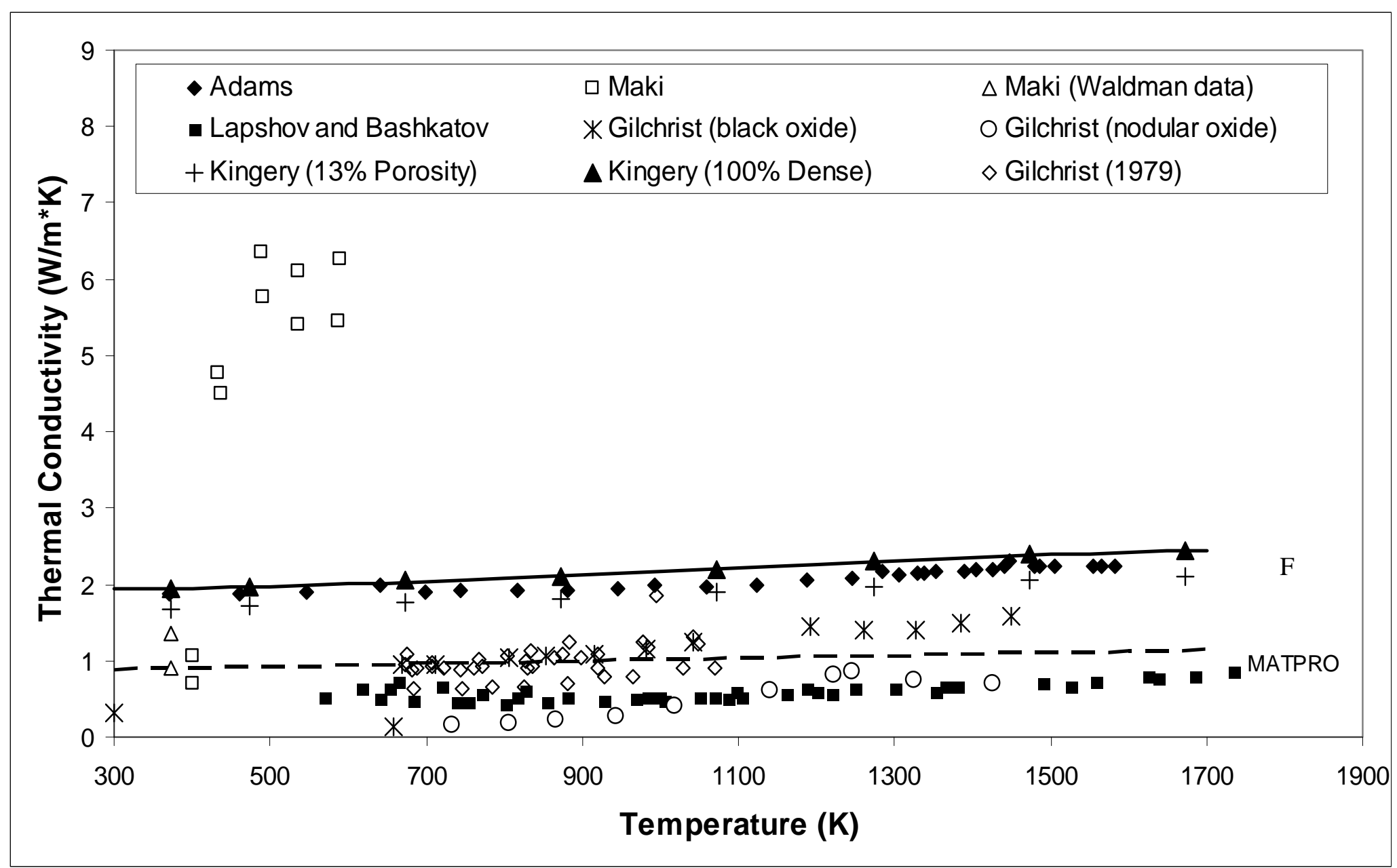

Figure 3.3-1 Oxide thermal conductivity measurements as a function of temperature compared with the MATPRO and FRAPCON3.4/FRAPTRAN 1.4 (FRAP) cladding oxide thermal conductivity models. 


\subsubsection{Applicability and Uncertainty}

The subroutine ZOTCON is applicable over the temperature range for which the data was collected, 375 to $1673 \mathrm{~K}$. Since the relatively high density of the bulk $\mathrm{ZrO}_{2}$ materials bias the thermal conductivity measurements high, it is difficult to establish a meaningful measurement of uncertainty.

The MATPRO model is applicable from 300K to the melting temperature (2973K). Upon melting the thermal conductivity, $\mathrm{K}_{\text {Liquid }}(\mathrm{W} / \mathrm{m} * \mathrm{~K})$, is assumed to be described by Equation 3.3-3 (Hagrman et al., 1981).

$$
\mathrm{K}_{\text {Liquid }}=1.4
$$

The standard error for the MATPRO model is approximately $\pm 0.75 \mathrm{~W} / \mathrm{m} * \mathrm{~K}$, but is assumed to be within 10 percent for materials that are known to be dense (>87\%TD) $\mathrm{ZrO}_{2}$.

\subsubsection{References}

Adams, M. 1954. “Thermal Conductivity: III, Prolate Spheroidal Envelope Method,” Journal of the American Ceramic Society, 37:74-79.

Maki, H. 1973. "Heat Transfer Characteristics of Zircaloy-2 Oxide Film,” Journal of Nuclear Science and Technology, 10:107-175.

Lapshov, V. N., and A. V. Bashkatov. 1973. "Thermal Conductivity of Coatings of Zirconium Dioxide Applied by the Plasma Sputtering Method,” Heat Transfer-Soviet Research, 5:19-22.

Gilchrist, K. E. 1976. “Thermal Property Measurements on Zircaloy-2 and Associated Oxide Layers,” Journal of Nuclear Materials, 62:257-264.

Kingery, W.D., et al. 1954. "Thermal Conductivity: X, Data for Several Pure Oxide Materials Corrected to Zero Porosity,” Journal of the American Ceramic Society, 37:107-100.

Hagrman, D.L., et al. 1981. MATPRO-Version 11 (Revision 2). A Handbook of Materials Properties for Use in the Analysis of Light Water Reactor Fuel Rod Behavior, NUREG/CR-0479 (TREE-1280, Rev. 2), EG\&G Idaho, Inc., Idaho Falls, ID.

Gilchrist, K.E. 1979. “Thermal Conductivity of Oxide Deposited on Zircaloy Fuel Tube Material - A Continuation of Previous Work,” Journal of Nuclear Materials, 82:193-194.

\subsection{Cladding Surface Emissivity (ZOEMIS)}

The subcode ZOEMIS returns the cladding surface emissivity, which is directly proportional to the radiant heat transfer from the cladding surface during an abnormal transient. The ZOEMIS model described in MATPRO is the same as the model used in FRAPCON-3.4 and FRAPTRAN 1.4 . 


\subsubsection{Model Development and Comparisons}

Surface emissivities are significantly affected by surface layers on the cladding. For cladding with thin oxide coatings, the oxide surface thickness is only a few wavelengths of near infrared radiation and is partly transparent. Oxide thickness is an important parameter for these thin coatings. Thicker oxide layers are opaque, so the oxide thickness is not as important as the nature of the outer oxide surface, which is affected by temperature and by chemical environment. The effect of temperature has been modeled, but variations in crud on the external cladding surface and chemical reaction products on the inside surface are not modeled explicitly.

The model for emissivity was constructed by considering measured emissivities reported by several investigators. Expressions used to predict the emissivity of Zircaloy cladding surfaces are summarized below.

When the cladding surface temperature has not exceeded 1500K, emissivities are modeled by Equations 3.4-1 and 3.4-2. Equation 3.4-1 is used for oxide layers less than $3.88 \times 10^{-6} \mathrm{~m}$ thick and Equation 3.4-2 is used for oxide layers equal to or greater than $3.88 \times 10^{-6} \mathrm{~m}$ thick. Both equations relate the hemispherical emissivity, $\varepsilon_{1}$ (unitless), to the oxide layer thickness, $\mathrm{d}(\mathrm{m})$.

$$
\begin{gathered}
\varepsilon_{1}=0.325+0.1246 \times 10^{6} \mathrm{~d} \\
\varepsilon_{1}=0.808642-50.0 \mathrm{~d}
\end{gathered}
$$

When the maximum cladding temperature has exceeded $1500 \mathrm{~K}$, emissivity is taken to be the larger of 0.325 and the result of Equation 3.4-3. Equation 3.4-3 relates the emissivity above $1500 \mathrm{~K}, \varepsilon_{2}$ (unitless), to $\varepsilon_{1}$ and the maximum cladding temperature, $\mathrm{T}(\mathrm{K})$.

$$
\varepsilon_{2}=\varepsilon_{1} \exp \left[\frac{1500-\mathrm{T}}{300}\right]
$$

Comparisons between data and the high and low temperature models are presented in Figure 3.4-1. 


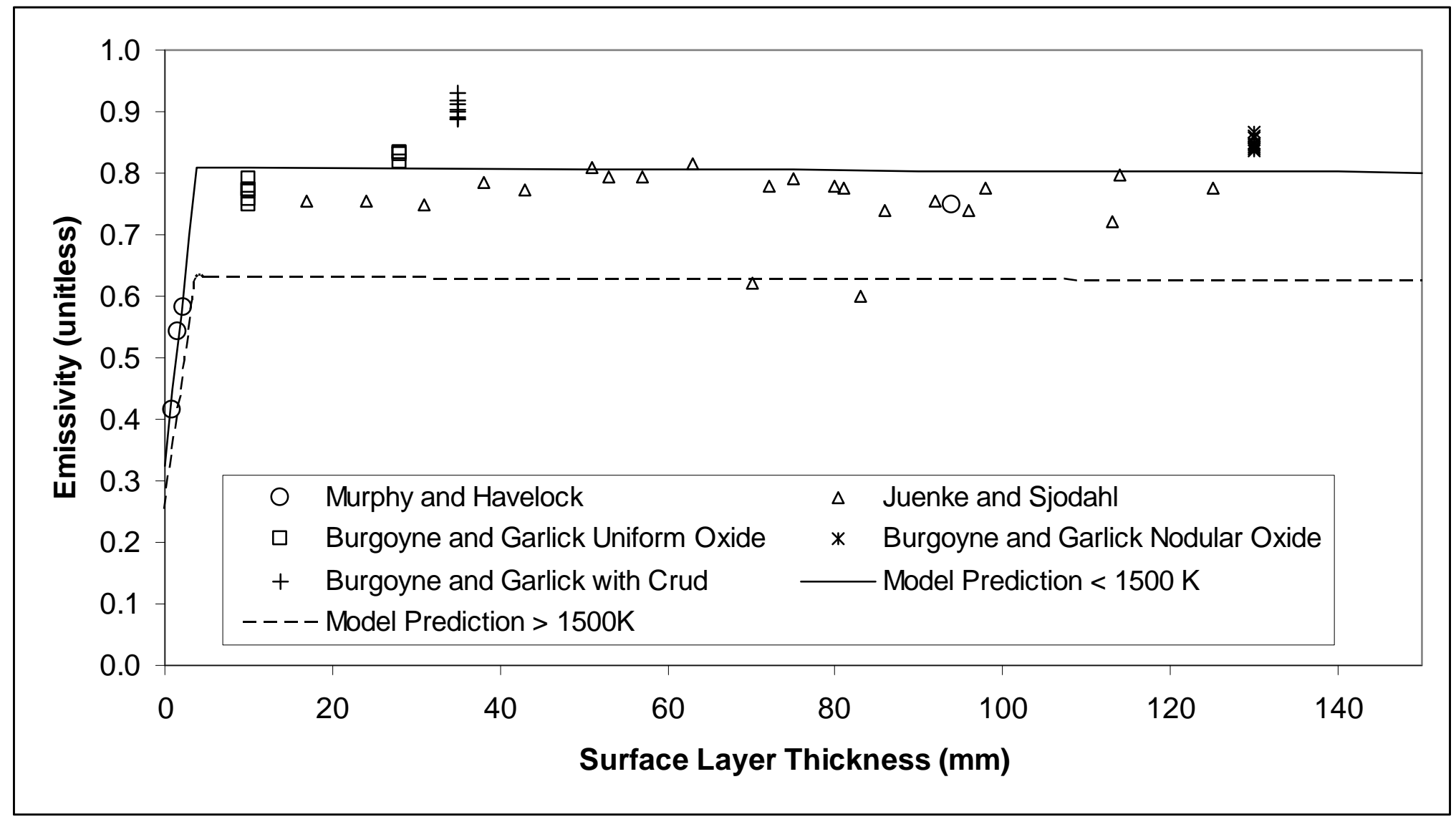

Figure 3.4-1 Model-to-data comparison for cladding oxide emissivity. 


\subsubsection{Applicability and Uncertainty}

The absolute standard error, $\sigma_{1}$, expected from the use for Equation 3.4-1 to predict emissivity in a reactor when cladding surface temperature has never exceeded $1500 \mathrm{~K}$ is given by Equation 3.4-4.

$$
\sigma_{1}= \pm 0.1
$$

When the maximum cladding temperature exceeds $1500 \mathrm{~K}$, the expected standard error is estimated by $\sigma_{2}$, which is described by Equation 3.4-5.

$$
\sigma_{2}= \pm 0.1 \exp \left[\frac{\mathrm{T}-1500}{300}\right]
$$

If Equations 3.4-3 and 3.4-5 predict values of $\varepsilon_{2}$ and $\sigma_{2}$ that fall inside the range of physically possible values of emissivity $(0.0-1.0)$, the value $\sigma_{2}$ is returned as the expected standard error. If the prediction $\varepsilon_{2}+\sigma_{2}$ is greater than 1 or if $\varepsilon_{2}-\sigma_{2}$ is less than 0 , the standard error of Equation 3.4-5 is modified to limit $\varepsilon_{2}+\sigma_{2}$ at 1 and/or $\varepsilon_{2}-\sigma_{2}$ at 0 .

\subsubsection{References}

Juenke, E.F., and L.H. Sjodahl. 1968. "Physical and Mechanical Properties: Emittance Measurements,” Advanced Pressure Vessel Materials, GEMP-1008, pp. 239-242, General Electric Co., Missile and Space Division, Cincinnati, OH.

Murphy, E.V., and F. Havelock. 1976. "Emissivity of Zirconium Alloys in Air in the Temperature Range 100-400 C,” Journal of Nuclear Materials, 60:167-176.

Burgoyne, T.B. and A. Garlick. 1976. "The effect of oxidation and crud deposition on the emissivity of Zircaloy-2 cladding." Paper presented to the OECD-CSNI Meeting on the Behavior of Water Reactor Fuel Elements under Accident Conditions, Spåtnid, Norway, Sept. 13-16, 1976.

\subsection{Cladding Thermal Expansion (CTHEXP)}

The subroutine CTHEXP returns the axial and diametral components of thermal expansion in the cladding as a function of temperature. The MATPRO model calculates these strain components for single crystal Zircaloy and applies these to polycrystalline cladding materials through the use of pole figures collected over a cladding section. The FRAPCON-3.4/FRAPTRAN 1.4 models use the correlation from Mehan and Wiesinger (1961) for temperatures below 1073K and a constant value of thermal expansion above 1273K. Values of thermal expansion between 1073 and $1273 \mathrm{~K}$ are determined in the FRAPCON-3.4/FRAPTRAN 1.4 codes by linear interpolation. In addition, the FRAPTAN 1.4 model includes a calculation of the thermal expansion for ZrNb-1.

\subsubsection{Model Development and Comparisons}

In the MATPRO model, a total of six correlations that are functions of temperature only are used to find single crystal thermal strains. In addition, basal plane symmetry $\left(\varepsilon_{11}=\varepsilon_{22}\right)$ is assumed. 
The model was developed for as fabricated Zircaloy-4, but comparisons with Zircaloy-2 and zirconium data also show good agreement for these materials.

The correlations for single crystal thermal strains are:

For $300<\mathrm{T}<1083 \mathrm{~K}$

$$
\begin{aligned}
& \varepsilon_{11}=4.95 \times 10^{-6} \mathrm{~T}-1.485 \times 10^{-3} \\
& \varepsilon_{33}=1.26 \times 10^{-5} \mathrm{~T}-3.78 \times 10^{-3}
\end{aligned}
$$

where

$\varepsilon_{11}=\quad$ circumferential thermal expansion $(\mathrm{m} / \mathrm{m})$

$\varepsilon_{33}=$ axial thermal expansion $(\mathrm{m} / \mathrm{m})$

For $1083 \leq \mathrm{T}<1244 \mathrm{~K}$

$$
\begin{aligned}
& \varepsilon_{11}=\left[2.77763+1.09822 \cos \left(\frac{\mathrm{T}-1083}{161} \pi\right)\right] \times 10^{-3} \\
& \varepsilon_{33}=\left[2.77763+1.09822 \cos \left(\frac{\mathrm{T}-1083}{161} \pi\right)\right] \times 10^{-3}
\end{aligned}
$$

where the arguments of the cosines are in radians.

For $1244 \leq \mathrm{T}<2098 \mathrm{~K}$

$$
\begin{aligned}
& \varepsilon_{11}=9.7 \times 10^{-6} \mathrm{~T}-1.04 \times 10^{-2} \\
& \varepsilon_{33}=9.76 \times 10^{-6} \mathrm{~T}-4.4 \times 10^{-3}
\end{aligned}
$$

For temperatures $\geq 2098 \mathrm{~K}$, consideration of the volume change associated with melting is required. Since no data were found for the MATPRO model, a typical 2 percent volume increase at melt was assumed (Siefken et al., 2001). The expressions used for the thermal strain in liquid zirconium (temperatures $\geq 2098 \mathrm{~K}$ ) are thus:

$$
\varepsilon_{\mathrm{p}}=\frac{2}{3} \varepsilon_{11}+\frac{1}{3} \varepsilon_{33}+0.0067
$$

$\varepsilon_{\mathrm{p}} \quad=\quad$ thermal expansion strain in liquid Zircaloy $(\mathrm{m} / \mathrm{m})$

$\varepsilon_{11}=\quad$ circumferential thermal expansion strain of a single crystal of Zircaloy at 2098K $(\mathrm{m} / \mathrm{m})$

$\varepsilon_{33}=\quad$ axial thermal expansion strain of a single crystal of Zircaloy at $2098 \mathrm{~K}(\mathrm{~m} / \mathrm{m})$

where the $\varepsilon_{11}$ and $\varepsilon_{33}$ are calculated by Equations $3.5-3$ and 3.5-4, respectively. 
To obtain cladding strains from these single crystal strains, it is necessary to do a volume weighted averaging of crystalline orientation over the entire cladding section. Such an averaging requires the collection of pole figures and is described in greater detail in the MATPRO (2001) handbook. The input parameters for typical cladding are not readily available.

The model for cladding thermal expansion in the FRAPCON-3.4/FRAPTRAN (FRAP) codes is different from the MATPRO model but provides similar predictions of expansion ( $<6$ percent). The data for the FRAP correlation used from room temperature to 1273K was taken from Mehan and Wiesinger (1961), Scott (1965), and Kearns (1965). Above 1273K, the coefficient of thermal expansion is the constant value of $9.7 \times 10^{-6}$, as recommended by Lustman and Kerze (1955). Between 1073 and 1273K (approximately the alpha-beta transition range for Zircaloy), the thermal expansion components are determined by linear interpolation.

The correlations used to calculate the axial and diametral components of thermal expansion in the cladding between room temperature and 1073K are presented in Equations 3.5-8 and 3.5-9, respectively. Strain is given a function of temperature, $\mathrm{T}\left({ }^{\circ} \mathrm{C}\right)$.

$$
\begin{gathered}
\varepsilon_{\text {axial }}=-2.5060 \times 10^{-5}+4.4410 \times 10^{-6} \mathrm{~T} \\
\varepsilon_{\text {diametral }}=-2.3730 \times 10^{-5}+6.7210 \times 10^{-6} \mathrm{~T}
\end{gathered}
$$

The correlations used to calculate the axial and diametral components of thermal expansion in the cladding above $1273 \mathrm{~K}$ are presented in Equations 3.5-10 and 3.5-11, respectively. Strain is given a function of temperature, $\mathrm{T}\left({ }^{\circ} \mathrm{C}\right)$.

$$
\begin{gathered}
\varepsilon_{\text {axial }}=-8.300 \times 10^{-3}+9.70 \times 10^{-6} \mathrm{~T} \\
\varepsilon_{\text {diametral }}=-6.800 \times 10^{-5}+9.70 \times 10^{-6} \mathrm{~T}
\end{gathered}
$$

Model-to-data comparisons for the MATPRO and FRAPCON-3.4/FRAPTRAN 1.4 models are presented in Figures 3.5-1 and 3.5-2 for axial and diametral thermal expansion, respectively. These comparisons illustrate that the axial and diametral components of cladding thermal expansions are being underpredicted. These correlations will be examined further and possibly revised in a future revision of FRAPCON-3.4/FRAPTRAN 1.4. 


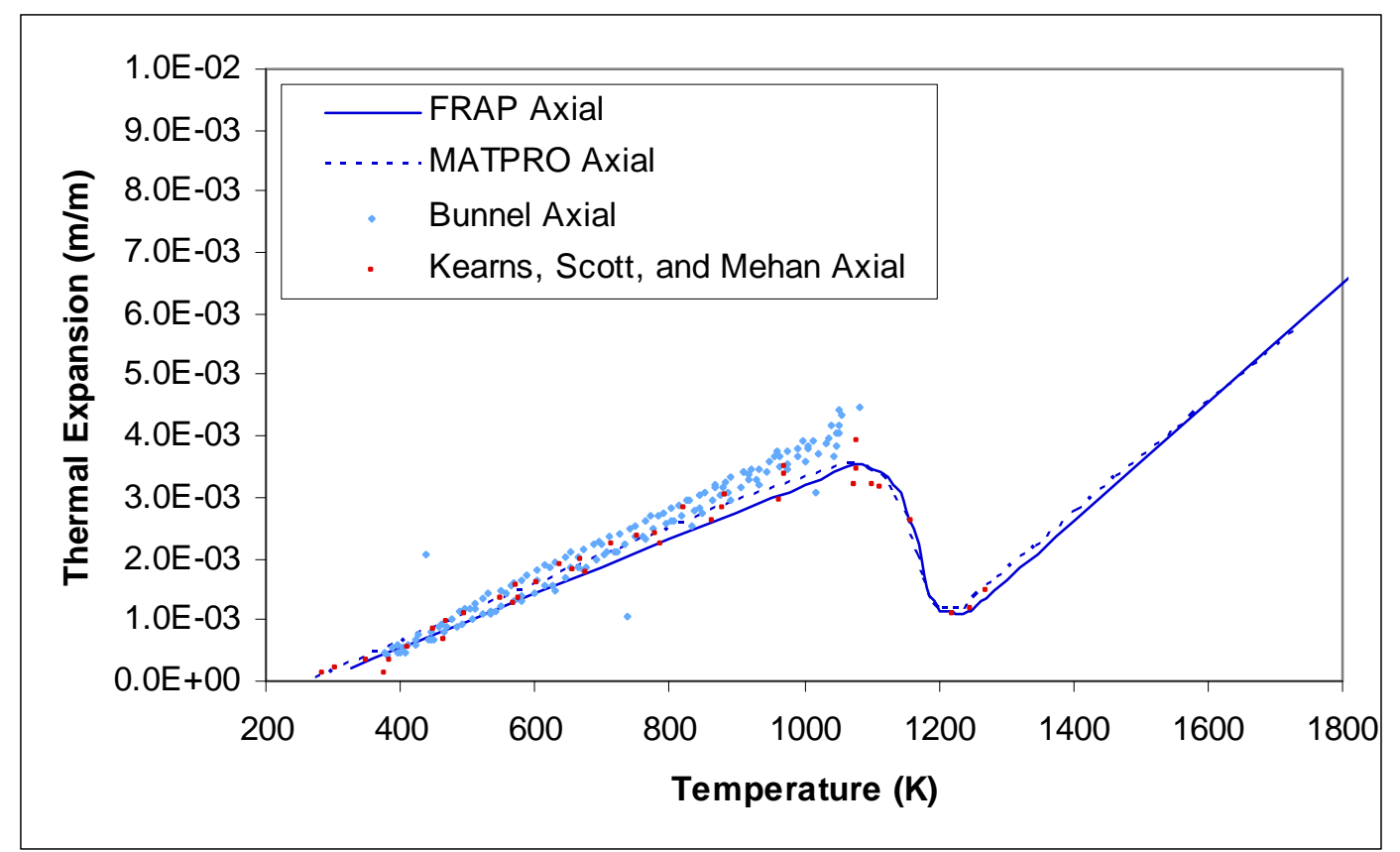

Figure 3.5-1 Model-to-data comparison for FRAPCON-3.4/FRAPTRAN 1.4 (FRAP) and MATPRO axial thermal expansion. Data from cladding tubes.

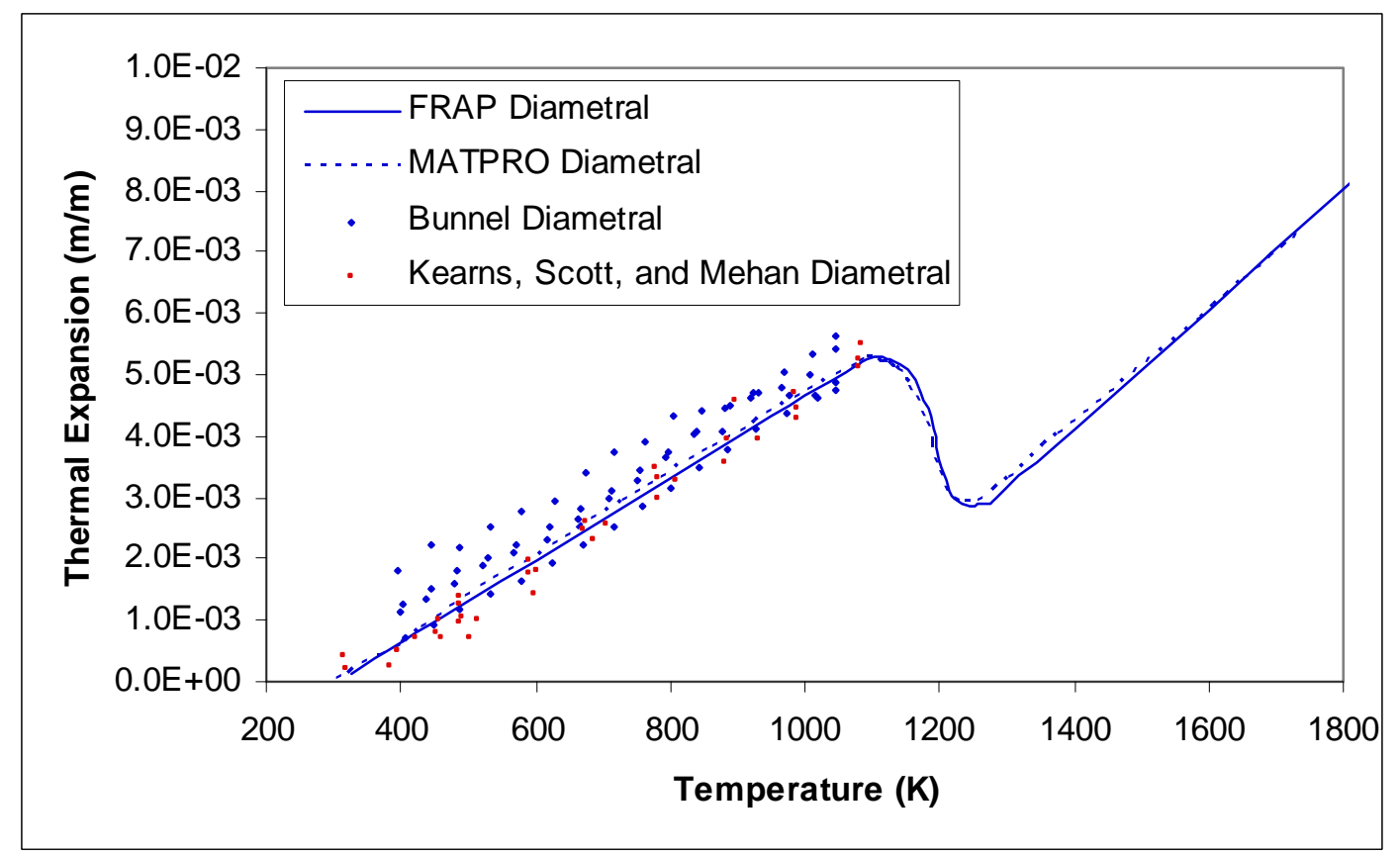

Figure 3.5-2 Model-to-data comparison for FRAPCON-3.4/FRAPTRAN 1.4 (FRAP) and MATPRO diametral thermal expansion. Data from cladding tubes.

\subsubsection{Applicability and Uncertainty}

The subroutine CTHEXP is applicable over the temperature range for which the experimental data was collected, which included temperatures up to $1100 \mathrm{~K}$. The uncertainty of the axial and diametral thermal expansion correlations has not been quantified. 


\subsubsection{References}

Mehan, R.L., and F.W. Wiesinger. 1961. Mechanical Properties of Zircaloy-2, KAPL-2110, General Electric Company, Knolls Atomic Power Laboratory, Schenectady, NY.

Siefken, L.J., E.W. Coryell, E.A. Harvego, and J.K. Hohorst. 2001. SCDAP/RELAP5/MOD 3.3 Code Manual MATPRO-A Library of Materials Properties for Light-Water-Reactor-Accident Analysis, NUREG/CR-6150, Vol. 4, Rev. 2, INEL-96/0422, Idaho National Engineering and Environmental Laboratory, Idaho Falls, ID.

Scott, P.B. 1965. Physical and Mechanical Properties of Zircaloy-2 and -4, WCAP-3269-41, Westinghouse Electric Corporation, Atomic Power Division, Pittsburgh, PA.

Kearns, J.J. 1965. Thermal Expansion and Preferred Orientation in Zircaloy, WAPD-TM-472, pp. 17-18, Bettis Atomic Power Laboratory, Pittsburgh, PA.

Lustman and F. Kerze. 1955. The Metallurgy of Zirconium, McGraw-Hill Book Company, New York, p. 355.

Bunnell, L.R., et al. 1977. High Temperature Properties of Zircaloy Oxygen Alloys, EPRI NP524, Battelle Pacific Northwest Laboratory, Richland, WA.

\subsection{Cladding Elastic Modulus (CELMOD) and Shear Modulus (CSHEAR)}

Elastic moduli are required to relate stresses to strains. The elastic moduli are defined by the generalized form of Hooke's law as elements of the fourth rank tensor that relates the second rank stress and strain tensors below the yield point. In practice, cladding is frequently assumed to be an isotropic material. In such a case, only two independent elastic moduli are needed to describe the relation between elastic stress and strain: the Young's modulus and the shear modulus.

The subcodes CELMOD and CSHEAR are used in the MATPRO and the FRAPCON-3.4/FRAPTRAN 1.4 (FRAP) codes to determine the Young's modulus and the shear modulus. The tensor from which these moduli are derived is calculated by CELAST, which is included in MATPRO and determines the compliance matrix for isotropic cladding. However, CELAST is not used in the FRAP codes. The subcode CELAST would only be required if the Young's modulus and shear modulus for an anisotropic cladding is desired and, as stated previously, the cladding is assumed to be isotropic.

Elastic moduli are affected primarily by temperature and oxygen content. Fast neutron fluence, cold work, and texture effects are also included in the models described herein, but they are not as important as temperature and oxygen content for typical LWR fuel rod cladding. The models are based primarily on data published by Bunnell et al. (1977), Fisher and Renken (1964), Armstrong and Brown (1964), and Padel and Groff (1976), since these data include the best description of texture for the temperature range in which they were used. Data from several other sources are used to evaluate the expected standard error of the CELMOD and CSHEAR codes and to estimate the effect of fast neutron fluence (Shober, 1957; Whitmarsh, 1962). To calculate Zircaloy elastic moduli at temperatures greater than the melting temperature of Zircaloy (2098K), the moduli are set to zero (actually, $1.0 \times 10^{-10}$ is used to avoid dividing by zero). 


\subsubsection{Model Development and Comparisons}

\section{$\underline{\text { CELMOD }}$}

The CELMOD subcode used in MATPRO is similar to the one used in FRAPCON-3.4/FRAPTRAN 1.4 (FRAP). The differences between the codes will be discussed in this subsection.

MATPRO uses Equations 3.6-1 and 3.6-2 to describe the Young's modulus in the alpha phase and the beta phase, respectively.

$$
\begin{gathered}
\mathrm{Y}=\left(1.088 \times 10^{11}-5.475 \times 10^{7} \mathrm{~T}+\mathrm{K}_{1}+\mathrm{K}_{2}\right) / \mathrm{K}_{3} \\
\mathrm{Y}=9.21 \times 10^{10}-4.05 \times 10^{7} \mathrm{~T}
\end{gathered}
$$

where

$\begin{array}{lll}\mathrm{Y} & = & \text { Young's modulus for Zircaloy-2 and }-4 \text { with random texture }(\mathrm{Pa}) \\ \mathrm{T} & = & \text { cladding temperature }(\mathrm{K}) \\ \mathrm{K}_{1} & = & \text { modification to account for the effect of oxidation }(\mathrm{Pa}) \\ \mathrm{K}_{2} & = & \text { modification to account for the effect of cold work }(\mathrm{Pa}) \\ \mathrm{K}_{3} & = & \text { modification to account for the effect of fast neutron fluence (unitless) }\end{array}$

In the alpha plus beta phase, MATPRO determines Y by linearly interpolating between values calculated at the alpha to alpha plus beta and the alpha plus beta to beta phase boundaries.

The MATPRO expressions used to model the effects of oxidation, cold work, and fast neutron fluence are presented in Equations 3.6-3 through 3.6-5.

$$
\begin{gathered}
\mathrm{K}_{1}=\left(6.61 \times 10^{11}+5.912 \times 10^{8} \mathrm{~T}\right) \Delta \\
\mathrm{K}_{2}=-2.6 \times 10^{10} \mathrm{C} \\
\mathrm{K}_{3}=0.88+0.12 \exp \left(-\frac{\Phi}{10^{25}}\right)
\end{gathered}
$$

where
$\Delta \quad=$
average oxygen concentration minus oxygen concentration of as-received cladding (kg oxygen/kg Zircaloy). As-received oxygen concentrations are so small (0.0012 kg oxygen $/ \mathrm{kg}$ Zircaloy) that the exact magnitude of the as-received concentration will not affect the correlation predictions.
$\mathrm{C}=\quad$ cold work (unitless ratio of areas).
$\Phi=$ fast neutron fluence $\left(\mathrm{n} / \mathrm{m}^{2}\right)$.

The standard error of CELMOD is $6.4 \times 10^{9} \mathrm{~Pa}$. Although FRAPCON-3.4 is coded to include this error, this value is not used. This standard error is not included in FRAPTRAN 1.4. 
The CELMOD subcode included in the FRAP codes differs slightly from the MATPRO version in several ways. For instance, the expression for Young's modulus in the alpha phase is presented in Equation 3.6-6.

$$
\text { cel mod }=\left(1.088 \times 10^{11}-5.475 \times 10^{7} * \text { ctemp }+c_{1} * \text { deloxy }+c_{3} * \text { cwkf }\right) / c_{2}
$$

where

celmod $\quad=\quad$ Young's modulus $(\mathrm{Pa})$

ctemp $\quad=\quad$ cladding temperature $(\mathrm{K})$

deloxy $\quad=\quad$ input average oxygen concentration excluding oxide layer $(\mathrm{kg}$ oxygen $/ \mathrm{kg}$ Zircaloy)(hardwired to zero in FRAPCON-3.4/FRAPTRAN 1.4)

cwkf $\quad=\quad$ input effective cold work (unitless ratio of areas)

In Equation 3.6-6, $c_{1}, c_{2}$, and $c_{3}$ are expressions that account for oxygen content, cold work, and fast neutron fluence respectively.

Equations 3.6-7 through 3.6-9 describe the constants $c_{1}, c_{2}$, and $c_{3}$, respectively.

$$
\begin{aligned}
& \mathrm{c}_{1}=\left(1.16 \times 10^{11}+\text { ctemp }^{*} 1.037 \times 10^{8}\right) * 5.7015 \\
& \mathrm{c}_{2}=1.0 \\
& \mathrm{c}_{3}=-2.6 \times 10^{10}
\end{aligned}
$$

For neutron fluences greater than $1 \times 10^{22}, c_{2}$ is given by Equation 3.6-10.

where

$$
c_{2}=0.88 *\left(1-\exp \left(- \text { fnck } / 1 \times 10^{25}\right)\right)+\exp \left(- \text { fnck } / 1 \times 10^{25}\right)
$$

fnck $=$ input effective fast fluence $\left(\mathrm{n} / \mathrm{m}^{2}\right)$

Both FRAPCON-3.4 and FRAPTRAN 1.4 use the same correlations to determine Young's modulus in the subcode CELMOD. Although FRAPCON-3.4 includes the coding to return the standard error of the model, neither FRAPCON-3.4 nor FRAPTRAN 1.4 uses these errors. FRAPTRAN 1.4 includes additional coding to determine Young's modulus for ZrNb-1.

\section{CSHEAR}

In MATPRO, the expressions used to determine shear modulus in the alpha and beta phases are given in Equations 3.6-11 and 3.6-12, respectively.

$$
\begin{gathered}
G=\frac{\left(4.04 \times 10^{10}-2.168 \times 10^{7} \mathrm{~T}+\mathrm{K}_{1}+\mathrm{K}_{2}\right)}{\mathrm{K}_{3}} \\
\mathrm{G}=3.49 \times 10^{10}-1.66 \times 10^{7} \mathrm{~T}
\end{gathered}
$$

In the alpha plus beta phase, MATPRO determines $\mathrm{G}$ by linearly interpolating between values calculated at the alpha to alpha plus beta and the alpha plus beta to beta phase boundaries. 
The constants $\mathrm{K}_{1}, \mathrm{~K}_{2}$, and $\mathrm{K}_{3}$ have the same definitions as stated before in CELMOD. However, the expression used to model the effect of oxidation for shear modulus is given in Equation 3.6-13.

$$
\mathrm{K}_{1}=\left(7.07 \times 10^{11}+2.315 \times 10^{8} \mathrm{~T}\right) \Delta
$$

The standard error of the CSHEAR code is $9 \times 10^{9} \mathrm{~Pa}$.

The CSHEAR subcode included in the FRAP codes differs slightly from the MATPRO version in several ways. For instance, the expression for shear modulus in the alpha phase is presented in Equation 3.6-14.

$$
\text { cshear }=\left(4.04 \times 10^{10}-2.168 \times 10^{7} * \text { ctemp }+c_{1} * \text { deloxy }+c_{3} * \text { cwkf }\right) / c_{2}
$$

where

$\begin{array}{lll}\text { cshear } & = & \text { shear modulus }(\mathrm{Pa}) \\ \text { ctemp } & = & \text { cladding temperature }(\mathrm{K}) \\ \text { deloxy } & = & \text { input average oxygen concentration excluding oxide layer } \\ \text { cwkf } & = & \text { input effective cold work (unitless ratio of areas) }\end{array}$

In Equation 3.6-14, $c_{1}, c_{2}$, and $c_{3}$ are expressions that account for oxygen content, cold work, and fast neutron fluence, respectively.

Equations 3.6-15 through 3.6-17 describe the constants $c_{1}, c_{2}$, and $c_{3}$, respectively.

$$
\begin{gathered}
\mathrm{c}_{1}=7.07 \times 10^{11}-\text { ctemp }^{*} 2.315 \times 10^{8} \\
\mathrm{c}_{2}=1.0 \\
\mathrm{c}_{3}=-0.867 \times 10^{10}
\end{gathered}
$$

For neutron fluences greater than $1 \times 10^{22}, c_{2}$ is given by Equation 3.6-18.

where

$$
c_{2}=0.88 *\left(1-\exp \left(- \text { fnck } / 1 \times 10^{25}\right)\right)+\exp \left(- \text { fnck } / 1 \times 10^{25}\right)
$$

fnck $=$ input effective fast fluence $\left(\mathrm{n} / \mathrm{m}^{2}\right)$

Both FRAPCON-3.4 and FRAPTRAN 1.4 use the same correlations to determine Young's modulus in the subcode CSHEAR. Although FRAPCON-3.4 includes the coding to return the standard error of the model, neither FRAPCON-3.4 nor FRAPTRAN 1.4 returns the error. FRAPTRAN 1.4 includes additional coding to determine Young's modulus, shear modulus, and Poisson's ratio as a function of temperature for $\mathrm{ZrNb}-1$.

Since there is limited data available from shear modulus measurements, a model-to-data comparison for Young's modulus is presented. Figure 3.6-1 presents predicted vs. measured Young's modulus values for data sets used in the development of the CELMOD models as well as more recent data. 
The upper and lower bounds of Figure 3.6-1 represent an interval of $\pm 6.4 \mathrm{GPa}$. It may be noted that the data fit is slightly better below $80 \mathrm{GPa}$ ( $\sim 4$ percent) than above $80 \mathrm{GPa}$ ( $>5$ percent). This is attributed to the relatively low temperatures that were used to collect these data (e.g., a quarter of Bolmaro's data is collected at $<250^{\circ} \mathrm{F}$ ). Nevertheless the CELMOD model, in general, provides a satisfactory fit to the data within the temperature range it will be applied for predicting in-reactor performance $\left(>250^{\circ} \mathrm{F}\right)$. 


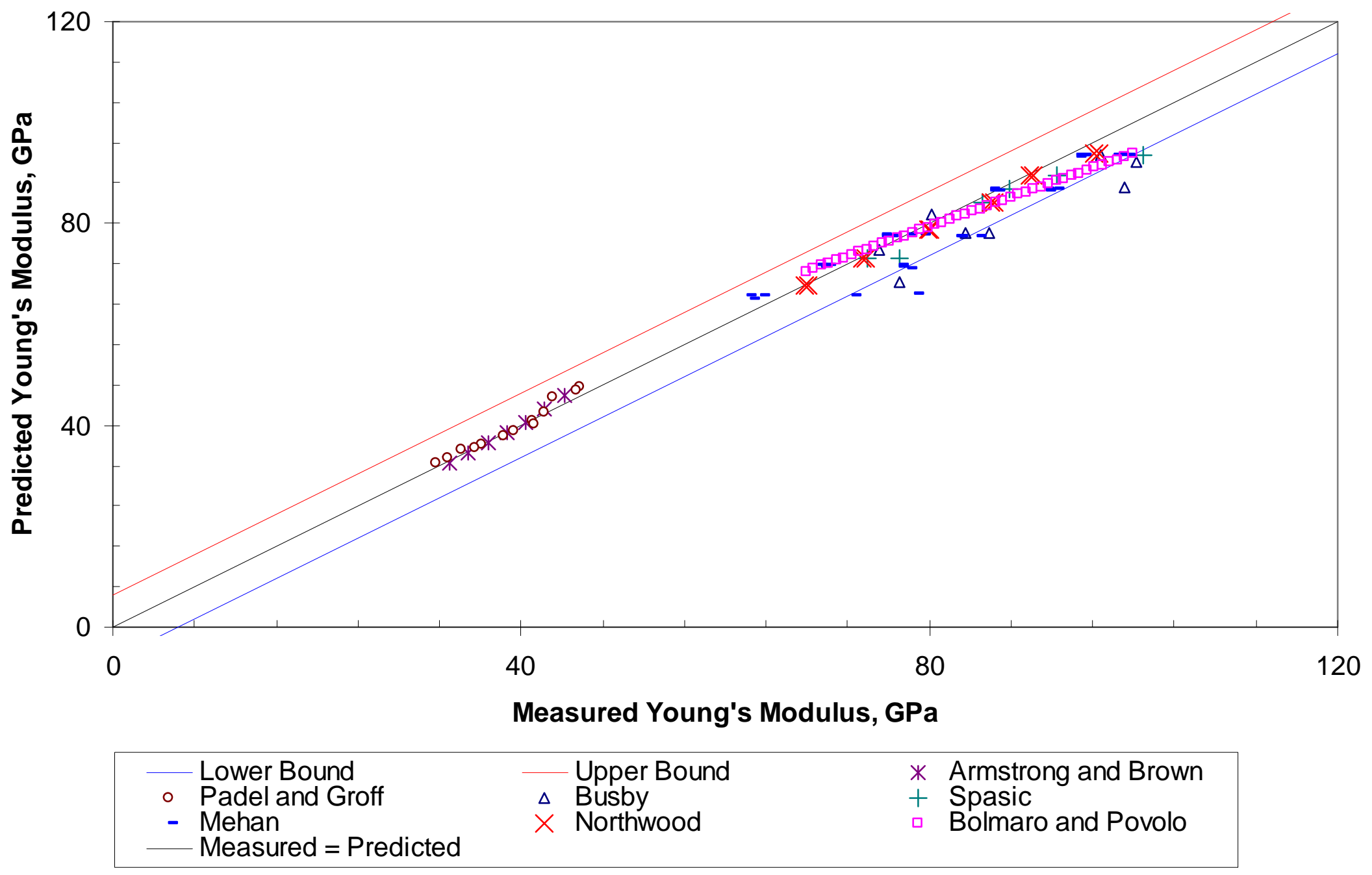

Figure 3.6-1 Predicted vs. measured values for Young's modulus for various data sets. 


\subsubsection{Applicability and Uncertainty}

The CELMOD and CSHEAR correlations are applicable below the melting temperature of the cladding (2098K). Above this temperature, the moduli are assumed to be zero (but actually set at $1 \times 10^{-10}$ to avoid dividing by zero). The CELMOD and CSHEAR correlations are similar between MATPRO and the FRAPCON-3.4/FRAPTRAN 1.4. The uncertainty of the predictions for CELMOD are within $6.4 \mathrm{GPa}$ (the upper and lower bounds), and typically $<5$ percent below $80 \mathrm{GWd} / \mathrm{MTU}$. The uncertainty of the CSHEAR predictions is estimated to be within $9 \mathrm{GPa}$.

\subsubsection{References}

Bunnell, L.R., et al. 1977. High Temperature Properties of Zircaloy Oxygen Alloys, EPRI NP524, Battelle Pacific Northwest Laboratory, Richland, WA.

Fisher, E.S., and C.J. Renken. 1964. "Single - Crystal Elastic Moduli and the CHP-BCC Transformation in Ti, Zr, and Hf,” Physical Review, 135(2A):A482-494.

Armstrong, P.E., and H.L. Brown. 1964. "Dynamic Young's Modulus Measurement above $1000^{\circ} \mathrm{C}$ on Some Pure Polycrystalline Metals and Commercial Graphites,” Transactions of the Metallurgical Society of AIME 230, August 1964, pp. 962-966.

Padel, A., and A. Groff. 1976. "Variation du Module de Young du Zirconium b en Function de la Temperature,” Journal of Nuclear Materials 59:325-326.

Shober, F.B., et al. 1957. The Mechanical Properties of Zirconium and Zircaloy-2, BMI-1168, Battelle Memorial Institute, Columbus, $\mathrm{OH}$.

Whitmarsh, C.L. 1962. Review of Zircaloy-2 and Zircaloy-4 Properties Relevant to N.S. Savannah Reactor Design, ORNL-3281, Oak Ridge National Laboratory, Oak Ridge, TN.

Mehan, R.L. 1958. Modulus of Elasticity of Zircaloy-2 Between Room Temperature and $1,000^{\circ} \mathrm{F}, \mathrm{KAPL}-\mathrm{M}-\mathrm{RLM}-16$, General Electric Company, Knolls Atomic Power Laboratory, Schenectady, NY.

Mehan, R.L., and F.W. Wiesinger. 1961. Mechanical Properties of Zricaloy-2, KAPL-2110, pp. 11-12, General Electric Company, Knolls Atomic Power Laboratory, Schenectady, NY.

Busby, C.C. 1966. Properties of Zircaloy-4 Tubing, WAPD-TM-585, p. 65, Bettis Atomic Power Laboratory, Pittsburgh, PA.

Northwood, D.O., et al. 1975. “Elastic Constants of Zirconium Alloys,” Journal of Nuclear Materials, 55:299-310.

Spasic, Z., et al. 1968. Conference on the Use of Zirconium Alloys in Nuclear Reactors, Marlanske Lanze, Czechoslovakia, USAEC CONF-681086, 1968, pp. 277-284.

Bolmaro, R.E., and F. Povolo. 1988. "Anelastic behaviour of materials under multiaxial strains," J. Materials Science, 23:371. 


\subsection{Cladding Axial Growth (CAGROW)}

The subcode CAGROW calculates the factional change in length of Zircaloy tubes due to irradiation-induced growth. The change in length of commercial fuel rods due to irradiation growth is small. However, growth can be a significant fraction of the clearance between the rod and the top and bottom assembly nozzles. Contact with the nozzles can cause rods to bow and possibly fail at points where the rods contact each other. In addition, rod growth increases the internal void volume that impacts the rod internal pressure calculation.

The original CAGROW model used in MATPRO was modified in FRAPCON-3.4 to better describe the cladding axial growth at higher fluence. The MATPRO version of CAGROW was applied to both Zircaloy-2 and -4. The FRAPCON-3.4 version includes correlations for Zircaloy-2 and -4, M5, and ZIRLO. These correlations may be applied to either PWR or boiling water reactor (BWR) conditions. The CAGROW subroutine is not used in FRAPTRAN 1.4 because significant irradiation axial growth is not expected under the short time scales considered by FRAPTRAN 1.4.

\subsubsection{Model Development and Comparisons}

The MATPRO correlation used to describe cladding axial growth is presented in Equation 3.7-1. This equation has been developed to model the irradiation growth of Zircaloy tubes at temperature between 40 and $360^{\circ} \mathrm{C}$ (the normal range of cladding temperatures in LWRs).

$$
\Delta \mathrm{L} / \mathrm{L}=\mathrm{A}[\exp (240.8 / \mathrm{T})](\Phi \mathrm{t})^{1 / 2}\left(1-\mathrm{f}_{\mathrm{z}}\right)(1+0.02 \mathrm{CW})
$$

where

$\begin{array}{lll}\Delta \mathrm{L} / \mathrm{L} & = & \text { fractional change in length due to growth. } \\ \mathrm{A} & = & 1.407 \mathrm{x} 10^{-16}\left(\mathrm{n} / \mathrm{m}^{2}\right)^{1 / 2} . \\ \mathrm{T} & = & \text { cladding temperature }(\mathrm{K}) . \\ \Phi & = & \text { fast neutron flux }\left(\mathrm{n} / \mathrm{m}^{2} \mathrm{~s}\right)(\mathrm{E}>1.0 \mathrm{MeV}) . \\ \mathrm{t} & = & \text { time }(\mathrm{s}) .\end{array}$

Axial growth for temperature below $40^{\circ} \mathrm{C}$ is approximated by using $\mathrm{T}=40^{\circ} \mathrm{C}$ in Equation $3.7-1$ and growth above $360^{\circ} \mathrm{C}$ is approximated by using $\mathrm{T}=360^{\circ} \mathrm{C}$.

The MATPRO subroutine CAGROW was based on low-burnup, low-fluence data found in Harbottle (1970), Kreyns (1960), and Daniel (1971, 1972). Consequently, the CAGROW model was updated in FRAPCON-3.4 to better model axial growth under greater fluences ( $>1 \times 10^{25}$ $\mathrm{n} / \mathrm{m}^{2}$ ). A model proposed by Franklin (1982) that was based on high fluence PWR data was adopted for FRAPCON-3.4. This model is presented in Equation 3.7-2 and relates the axial growth, ax (m/m), of stress relief annealed (SRA) Zircaloy-4 in a PWR to fluence (n/ $\left.\mathrm{cm}^{2}\right)$.

$$
\mathrm{ax}=2.18 \times 10^{-21} * \Phi^{0.845}
$$


where

ax $\quad=\quad$ axial growth increment $(\mathrm{m} / \mathrm{m})$

$\Phi=$ fast neutron fluence $\left(\mathrm{n} / \mathrm{cm}^{2}\right)(\mathrm{E}>1.0 \mathrm{MeV})$

The expression in Equation 3.7-2 is used to determine the axial growth at the beginning and the end of a time increment. The difference is used to determine the axial growth for the time increment, which is the value returned by the CAGROW subroutine. The expression in Equation 3.7-2 can be used to describe the axial growth of fully recrystallized annealed (RXA) Zircaloy-2 cladding in a BWR by multiplying by a factor of 0.5 .

In addition to updating the cladding axial growth model for SRA Zircaloy-4 and RXA Zircaloy-2, new correlations have been added to describe the cladding axial growth of M5 and ZIRLO. The cladding axial growth correlations for M5 and ZIRLO are presented in Equations 3.7-3 and 3.7-4, respectively. These equations relate the cladding axial growth, ax $(\mathrm{m} / \mathrm{m})$, to fluence $\left(\mathrm{n} / \mathrm{cm}^{2}\right)$. The variables have the same definitions as those described in Equation 3.7-2 and the time difference is used to determine the axial growth during the time increment, which is the value returned by the CAGROW subroutine.

For M5 cladding:

$$
\mathrm{ax}=7.013 \times 10^{-21} \Phi^{0.81787}
$$

For ZIRLO cladding:

$$
\mathrm{ax}=9.7893 \times 10^{-25} \Phi^{0.98239}
$$

Model-to-data comparisons are presented in Figures 3.7-5 through 3.7-8 for SRA Zircaloy-4, RXA Zircaloy-2, M5, and ZIRLO, respectively. Based on these figures, it appears that the cladding axial growth of these alloys can be predicted well with the CAGROW subroutine. 


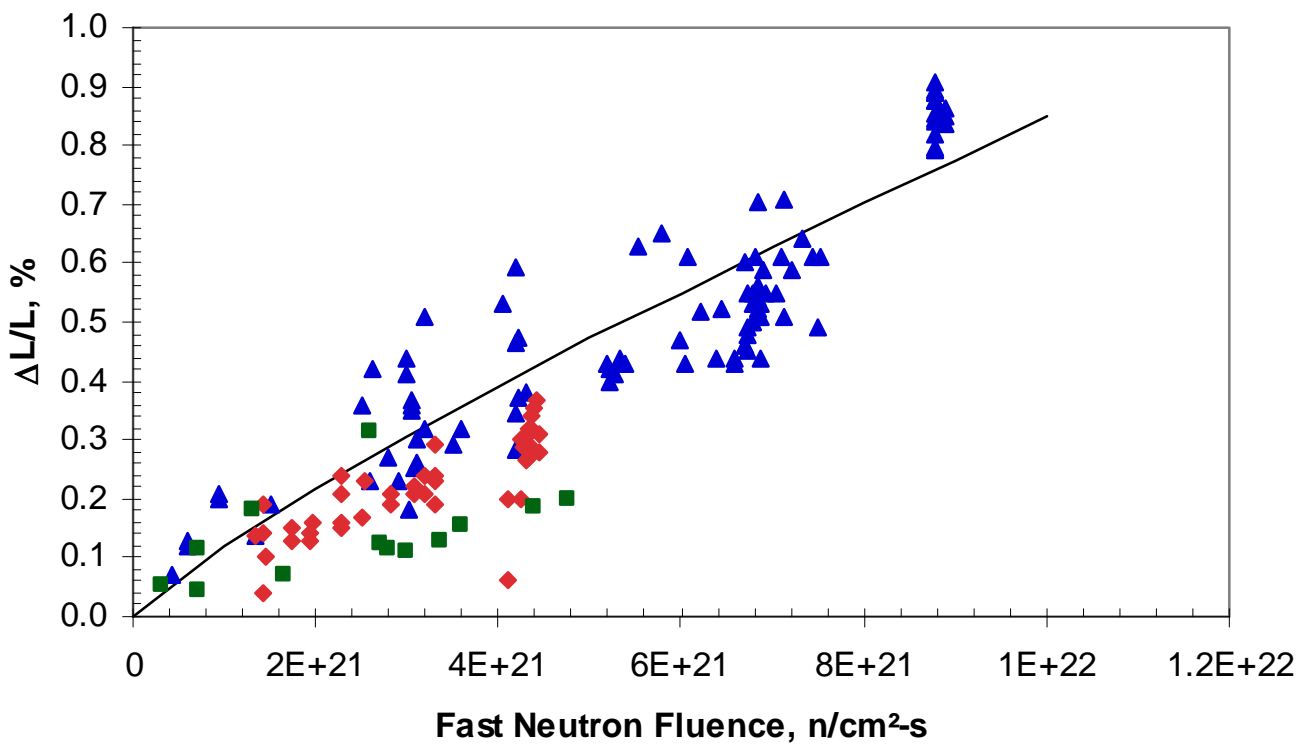

\ Newman - Franklin - Gilbon —Zr-4 PWR Model

Figure 3.7-5 Model-to-data comparison for SRA Zircaloy-4 (PWR). The standard error of $\Delta \mathrm{L} / \mathrm{L}(\%)$ is 0.11 .

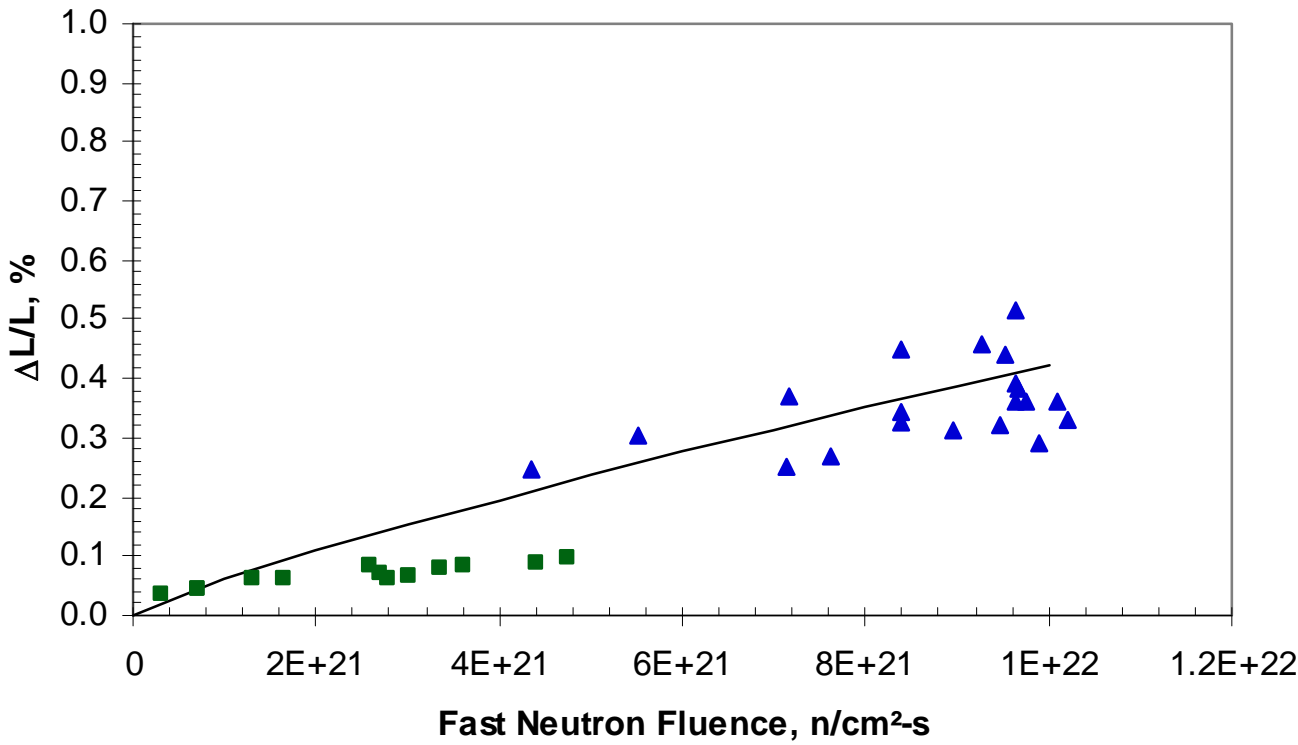

\ FRAPCON Data - Gilbon —Zr-2 BWR Model

Figure 3.7-6 Model-to-data comparison for RXA Zircaloy-2 (BWR). The standard error of $\Delta \mathrm{L} / \mathrm{L}(\%)$ is 0.07 . 


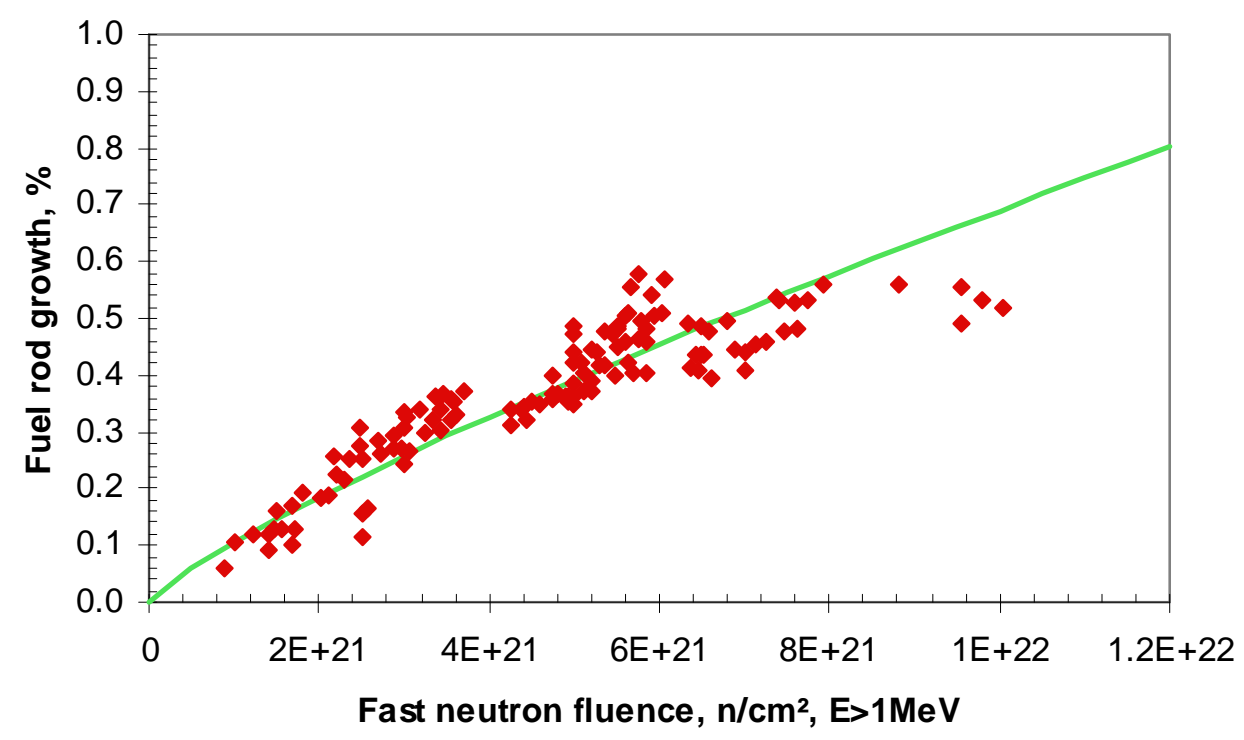

- M5 Data —PWR M5 Model

Figure 3.7-7 Model-to-data comparison for M5. M5 data was collected by Gilbon et al. (2000). The standard error of $\Delta \mathrm{L} / \mathrm{L}(\%)$ is 0.05 .

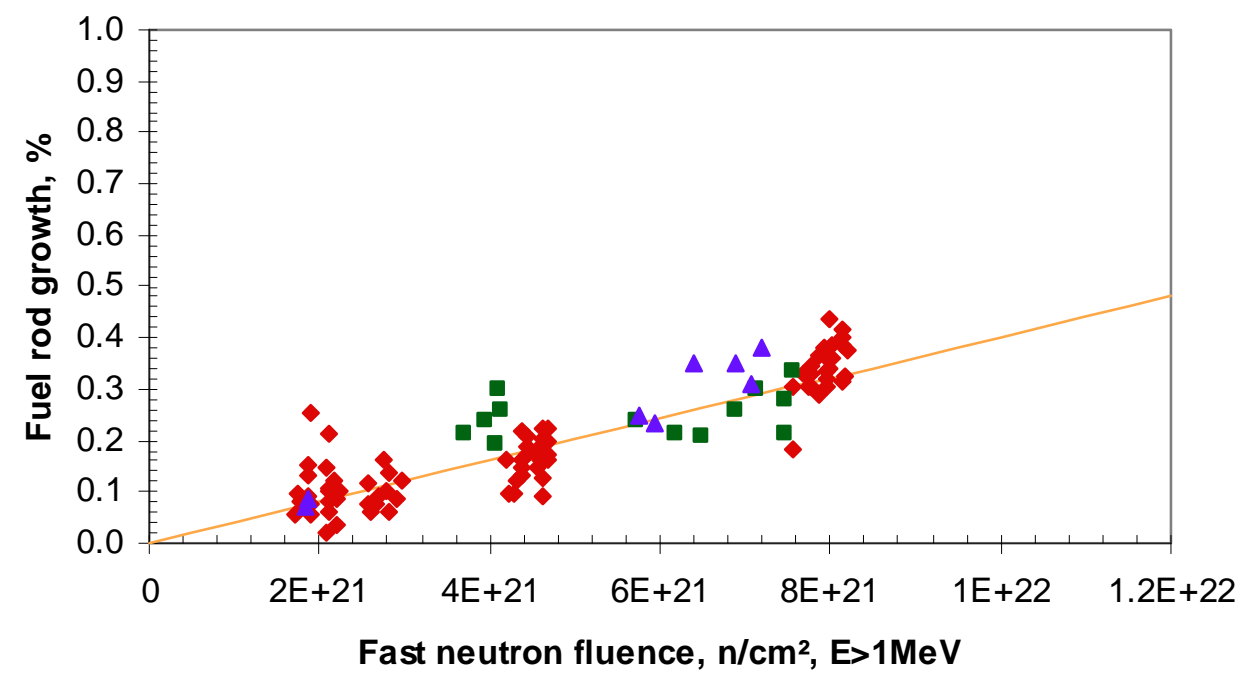
- Vandellos ZIRLO Data
- BR-3 ZIRLO Data
- North Anna ZIRLO Data PWR ZIRLO Model

Figure 3.7-8 Model-to-data comparison for ZIRLO. Vandellos data was collected by Irisa (2000) while North Anna and BR-3 data was collected by Sabol (1994). The standard error of $\Delta \mathrm{L} / \mathrm{L}(\%)$ is 0.05 . 


\subsubsection{Applicability and Uncertainty}

The CAGROW model in FRAPCON-3.4 is applicable over the temperature range from 700 to $900 \mathrm{~K}$ for local burnups up to $65 \mathrm{GWd} / \mathrm{MTU}$ (or $12 \times 10^{25} \mathrm{n} / \mathrm{m}^{2}$ fluence, $\mathrm{E}>1 \mathrm{MeV}$ ). The absolute uncertainties for the growth strains predicted for stress-relief annealed Zr-4, M5, and ZIRLO are $0.11,0.05$, and 0.05 , respectively. These three alloys are typically used in PWRs, whereas recrystallized $\mathrm{Zr}-2$ is used in BWRs. The absolute uncertainty in growth strains for $\mathrm{Zr}-2$ is 0.07 .

\subsubsection{References}

Harbottle, J.E. 1970. “The Temperature and Neutron Dose Dependence of Irradiaton Growth in Zircaloy-2," Irradiation Effect on Structural Alloys for Nuclear Reactor Applications, ASTMSTP-485, American Society of Testing and Materials, West Conshohocken, PA, pp. 287-299.

Kreyns, P.H., quoted by E. Duncombe et al. 1996. Comparison with Experiment of Calculated Changes and Failure Analysis of Irradiated Bulk Oxide Fuel Test Rods Using the CYGRO01 Computer Program, WAPD-TM-583, Bettis Atomic Power Laboratory, Pittsburgh, PA.

Daniel, R.C. 1971. In-Pile Dimensional Changes of Zircaloy-4 Tubing Having Low Hoop Stresses (LWBR Development Program), WAPD-TM-973, Bettis Atomic Power Laboratory, Pittsburgh, PA.

Daniel, R.C. 1972. "In-Pile Dimensional Changes of Zircaloy-4 Tubing Having Stresses (Light Water Breeder Reactor Development Program),” Nuclear Technology, 14:171-186.

Franklin, D.G. 1982. “Zircaloy Cladding Deformation during Power Reactor Irradiation.” In Proceedings of the Fifth International Symposium on Zirconium in the Nuclear Industry, ASTMSTP-754, American Society of Testing and Materials, West Conshohocken, PA, pp. 235-267.

Newman, L.W. 1986. The Hot Cell Examination of Oconee 1 Fuel Rods After Five Cycles of Irradiation, DOE/ET/34212-50, Babcock and Wilcox Company, Lynchburg, VA.

Gilbon, D., A. Soniak, S. Doriot, and J.P. Mardon. 2000. "Irradiation Creep and Growth Behavior, and Microstructural Evolution of Advanced Zr-Base Alloys," Zirconium in the Nuclear Industry: Twelfth International Symposium, ASTM-STP-1354, GP Sabol and GD Moan eds., American Society of Testing and Materials, West Conshohocken, PA, pp. 51-73.

Irisa, Y., Murai, K., Goto, K., Suzuki, S., Sabate, R., Alonso, J.M. 2000. "Segmented Fuel Rod Irradiation Programme on Advanced Materials for High Burnup,” in Proceedings of the 2000 Light Water Fuel Performance Meeting, Park City, UT, April 10-13, 2000.

Sabol, G.P., R.J. Comstock, R.A. Weiner, P. Larouere, and R.N. Stanutz. 1994. "In-Reactor Corrosion Performance of ZIRLO and Zircaloy-4,” Zirconium in the Nuclear Industry: Tenth International Symposium, ASTM-STP-1425, A.M. Garde and E.R. Bradley, eds. American Society for Testing and Materials, Philadelphia, PA, pp. 724-744. 


\subsection{Creep Rate (CREPR)}

The subcode CREPR is used by FRAPCON-3.4 to determine the cladding creep rate, accounting for both thermal and irradiation creep. The CREPR subcode was not used in MATPRO. Also, since creep is a time-dependent deformation process, CREPR is not used in FRAPTRAN 1.4, because creep occurs over much longer time intervals than those considered in FRAPTRAN 1.4 of a minute or less. The FRAPTRAN calculation of cladding ballooning as a result of a LOCA is handled with a separate subroutine named BALON2

\subsubsection{Model Development and Comparisons}

The creep model used by FRAPCON-3.4 is based on the model given by Limbäck and Andersson (1996). This model uses a thermal creep model described by Matsuo (1987) and an empirical irradiation creep rate with tuned model parameters that were fit to data by Franklin et al. (1983). The Limbäck model was further modified by PNNL to use effective stress rather than hoop stress as an input so that the difference in creep behavior during tensile and compressive creep would be modeled correctly. Several of the fitting coefficients were consequently changed to accommodate this modification. In addition, a temperature-dependent term was added to the formula for irradiation creep strain rate. This model has different parameters for SRA and RXA cladding types, and provides much more reasonable creep strains in the LWR range of temperatures and cladding hoop stresses than the previous model used in FRAPCON-3.3.

The steady-state thermal and irradiation creep rates are given by Equations 3.8-1 and 3.8-2, respectively.

$$
\begin{gathered}
\dot{\varepsilon}_{\text {th }}=A \frac{E}{T}\left(\sinh \frac{\mathrm{a}_{\mathrm{i}} \sigma_{\text {eff }}}{\mathrm{E}}\right)^{\mathrm{n}} \exp \left(\frac{-\mathrm{Q}}{\mathrm{RT}}\right) \\
\dot{\varepsilon}_{\mathrm{irr}}=\mathrm{C}_{\mathrm{o}} \cdot \phi^{\mathrm{C}_{1}} \cdot \sigma_{\mathrm{eff}}^{\mathrm{C}_{2}} \cdot \mathrm{f}(\mathrm{T})
\end{gathered}
$$

where

$\begin{array}{lll}\dot{\varepsilon}_{\text {th }} & = & \text { thermal strain rate (in./in./hr) } \\ \dot{\varepsilon}_{\text {irr }} & = & \text { irradiation strain rate (in./in./hr) } \\ \mathrm{T} & = & \text { temperature (K) } \\ \sigma_{\text {eff }} & = & \text { effective stress }(\mathrm{MPa}) \\ \mathrm{R} & = & \text { universal gas constant }(0.008314 \mathrm{~kJ} / \mathrm{mol}-\mathrm{K}) \\ \phi & = & \text { fast neutron flux }\left(\mathrm{n} / \mathrm{m}^{2}-\mathrm{s}\right)\end{array}$

The variables $A, E, a_{i}, n,-Q, C_{0}, C_{1}, C_{2}$, and the function $f(T)$ have different values depending on temperature, flux, and cladding type (SRA or RXA). The values for these variables under different conditions are presented in Table 3.8-1. 
Table 3.8-1 Parameters for FRAPCON-3.4 creep equation for SRA and RXA cladding.

\begin{tabular}{|c|c|c|c|}
\hline Parameter & Units & SRA Cladding & RXA Cladding \\
\hline A* & $\mathrm{K} / \mathrm{MPa} / \mathrm{hr}$ & $1.08 \times 10^{9} *$ & $5.47 \times 10^{8} *$ \\
\hline$E$ & $\mathrm{MPa}$ & \multicolumn{2}{|c|}{$1.149 \times 10^{5} * \mathrm{~T}$} \\
\hline $\mathrm{a}_{\mathrm{i}}$ & $\mathrm{MPa}^{-1}$ & \multicolumn{2}{|c|}{$\begin{array}{c}650\left\{1-0.56\left[1-\exp \left(-1.4 \times 10^{-27} * \Phi^{1.3}\right)\right]\right\} \\
\Phi=\text { fast neutron fluence }\left(\mathrm{n} / \mathrm{cm}^{2}\right)\end{array}$} \\
\hline $\mathrm{n}$ & unitless & 2.0 & 3.5 \\
\hline $\mathrm{Q}$ & $\mathrm{kJ} / \mathrm{mole}$ & \multicolumn{2}{|c|}{201} \\
\hline $\mathrm{C}_{\mathrm{o}}$ & $\begin{array}{l}\left(\mathrm{n} / \mathrm{m}^{2}-\mathrm{s}\right)^{-\mathrm{Cl}} \\
\mathrm{MPa}^{-\mathrm{C} 2}\end{array}$ & $4.0985 \times 10^{-24}$ & $1.87473 \times 10^{-24}$ \\
\hline $\mathrm{C}_{1}$ & unitless & \multicolumn{2}{|c|}{0.85} \\
\hline $\mathrm{C}_{2}$ & unitless & \multicolumn{2}{|c|}{1.0} \\
\hline$f(T)^{*}$ & unitless & $\begin{array}{ll}\mathrm{T}<570 & 0.7283 \\
570<\mathrm{T}<625 & -7.0237+0.0136 \mathrm{~T} \\
\mathrm{~T}>625 & 1.4763 \\
\end{array}$ & $\begin{array}{l}0.7994 \\
-3.18562+0.00699132 \mathrm{~T} \\
1.1840\end{array}$ \\
\hline
\end{tabular}

*Parameters changed from original Limbäck equation.

The thermal and irradiation creep rates may be added together as shown in Equation 3.8-3 and used to calculate the saturated primary hoop strain, which is presented in Equation 3.8-4.

$$
\begin{gathered}
\dot{\varepsilon}_{\text {th }+ \text { irr }}=\dot{\varepsilon}_{\text {th }}+\dot{\varepsilon}_{\text {irr }} \\
\varepsilon_{\mathrm{p}}^{\mathrm{s}}=0.0216 \cdot \dot{\varepsilon}_{\text {th }+ \text { irr }}^{0.109}\left(2-\tanh \left(35500 \cdot \dot{\varepsilon}_{\text {th }+ \text { irr }}\right)\right)^{-2.05}
\end{gathered}
$$

The total thermal strain can then be calculated as a function of time, $t$ (hours), as shown in Equation 3.8-5. However, in FRAPCON-3.4 the strain rate is used, which is obtained by taking the derivative of Equation 3.8-5. This derivative is presented in Equation 3.8-6, which relates the total thermal strain rate to the saturated primary hoop strain, the combined thermal and irradiation creep rates, and time, t, in hours.

$$
\begin{gathered}
\varepsilon_{\mathrm{H}}=\varepsilon_{\mathrm{p}}^{\mathrm{s}}\left(1-\exp \left(-52 \cdot \sqrt{\dot{\varepsilon}_{\mathrm{th}+\text { irr }} \cdot \mathrm{t}}\right)\right)+\dot{\varepsilon}_{\mathrm{th}+\mathrm{irr}} \cdot \mathrm{t} \\
\dot{\varepsilon}_{\mathrm{H}}=\frac{52 \cdot \varepsilon_{\mathrm{p}}^{\mathrm{s}} \cdot \dot{\varepsilon}_{\mathrm{th}+\mathrm{irr}}^{1 / 2}}{2 \cdot \mathrm{t}^{1 / 2}} \exp \left(-52 \cdot \sqrt{\dot{\varepsilon}_{\mathrm{th}+\text { irr }} \cdot \mathrm{t}}\right)+\dot{\varepsilon}_{\text {th }+ \text { irr }}
\end{gathered}
$$

The effective stress in the cladding is found using the principal stresses at the mid-wall radius using the thick wall formula. The principle stresses can be determined with Equations 3.8-7 through 3.8-9.

$$
\begin{gathered}
\sigma_{\mathrm{r}}=\frac{\mathrm{P}_{\mathrm{i}} \mathrm{r}_{\mathrm{i}}^{2}-\mathrm{P}_{\mathrm{o}} \mathrm{r}_{\mathrm{o}}^{2}+\frac{\mathrm{r}_{\mathrm{i}}^{2} \mathrm{r}_{\mathrm{o}}^{2}\left(\mathrm{P}_{\mathrm{o}}-\mathrm{P}_{\mathrm{i}}\right)}{\mathrm{r}^{2}}}{\mathrm{r}_{\mathrm{o}}^{2}-\mathrm{r}_{\mathrm{i}}^{2}} \\
\sigma_{\mathrm{t}}=\frac{\mathrm{P}_{\mathrm{i}} \mathrm{r}_{\mathrm{i}}^{2}-\mathrm{P}_{\mathrm{o}} \mathrm{r}_{\mathrm{o}}^{2}-\frac{\mathrm{r}_{\mathrm{i}}^{2} \mathrm{r}_{\mathrm{o}}^{2}\left(\mathrm{P}_{\mathrm{o}}-\mathrm{P}_{\mathrm{i}}\right)}{\mathrm{r}^{2}}}{\mathrm{r}_{\mathrm{o}}^{2}-\mathrm{r}_{\mathrm{i}}^{2}}
\end{gathered}
$$




$$
\sigma_{1}=\frac{P_{i} r_{i}^{2}-P_{o} r_{o}^{2}}{r_{o}^{2}-r_{i}^{2}}
$$

where

$\begin{array}{lll}\mathrm{P}_{\mathrm{i}} & = & \text { inner pressure } \\ \mathrm{P}_{\mathrm{o}} & = & \text { outer pressure } \\ \mathrm{r}_{\mathrm{i}} & = & \text { inner radius } \\ \mathrm{r}_{\mathrm{o}} & = & \text { outer radius } \\ \mathrm{r} & = & \text { radius within tube } \\ \sigma_{\mathrm{r}} & = & \text { radial stress } \\ \sigma_{\mathrm{t}} & = & \text { tangential stress } \\ \sigma_{\mathrm{l}} & = & \text { longitudinal stress }\end{array}$

The effective stress can then be calculated by Equation 3.8-10.

$$
\sigma_{\text {eff }}=\sqrt{0.5\left(\left(\sigma_{1}-\sigma_{t}\right)^{2}+\left(\sigma_{t}-\sigma_{r}\right)^{2}+\left(\sigma_{r}-\sigma_{l}\right)^{2}\right)}
$$

The subcode CREPR can be used for newer alloys like M5 and ZIRLO. It has been found that the Zircaloy RXA model adequately describes the creep behavior of M5 (Gilbon et al., 2000; Soniak et al., 2002). The Zircaloy SRA model is used for ZIRLO with a reduction factor of 0.8. The reduction factor is the result of studies that have shown that ZIRLO exhibits about 80 percent of the Zircaloy-4 creepdown (Sabol et al., 1994).

Model-to-data comparisons are presented in Figures 3.8-1 and 3.8-2 for SRA and RXA Zircaloy cladding. Creep data was obtained from irradiated SRA and RXA tube from Franklin (1983), Soniak et al. (2002), and Gilbon et al. (2000). The relative standard error for the predictions is 14.5 percent for SRA and 21.6 percent for RXA cladding. 


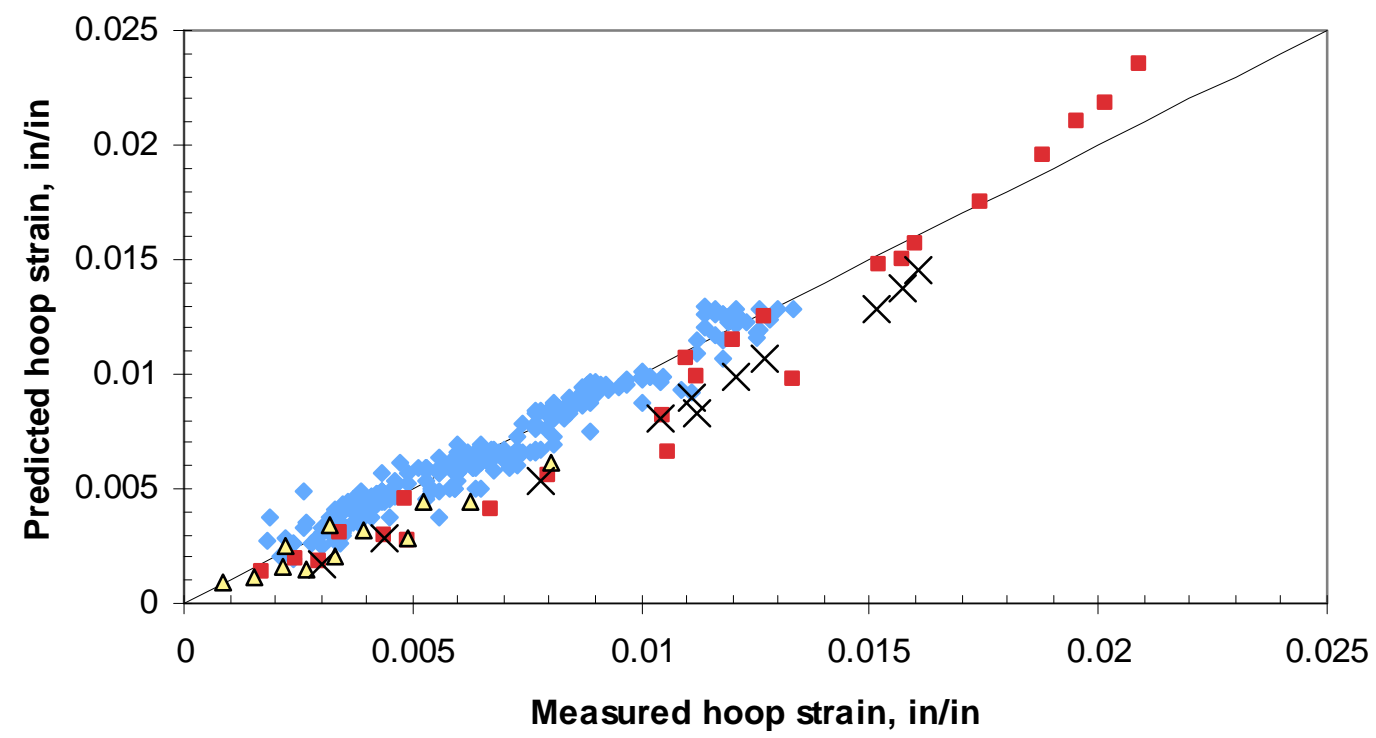

- Franklin - Soniak $350^{\circ} \mathrm{C} \Delta$ Soniak $320^{\circ} \mathrm{C} \times$ Gilbon — Predicted=Measured

Figure 3.8-1 Predicted vs. measured hoop strain for SRA Zircaloy model.

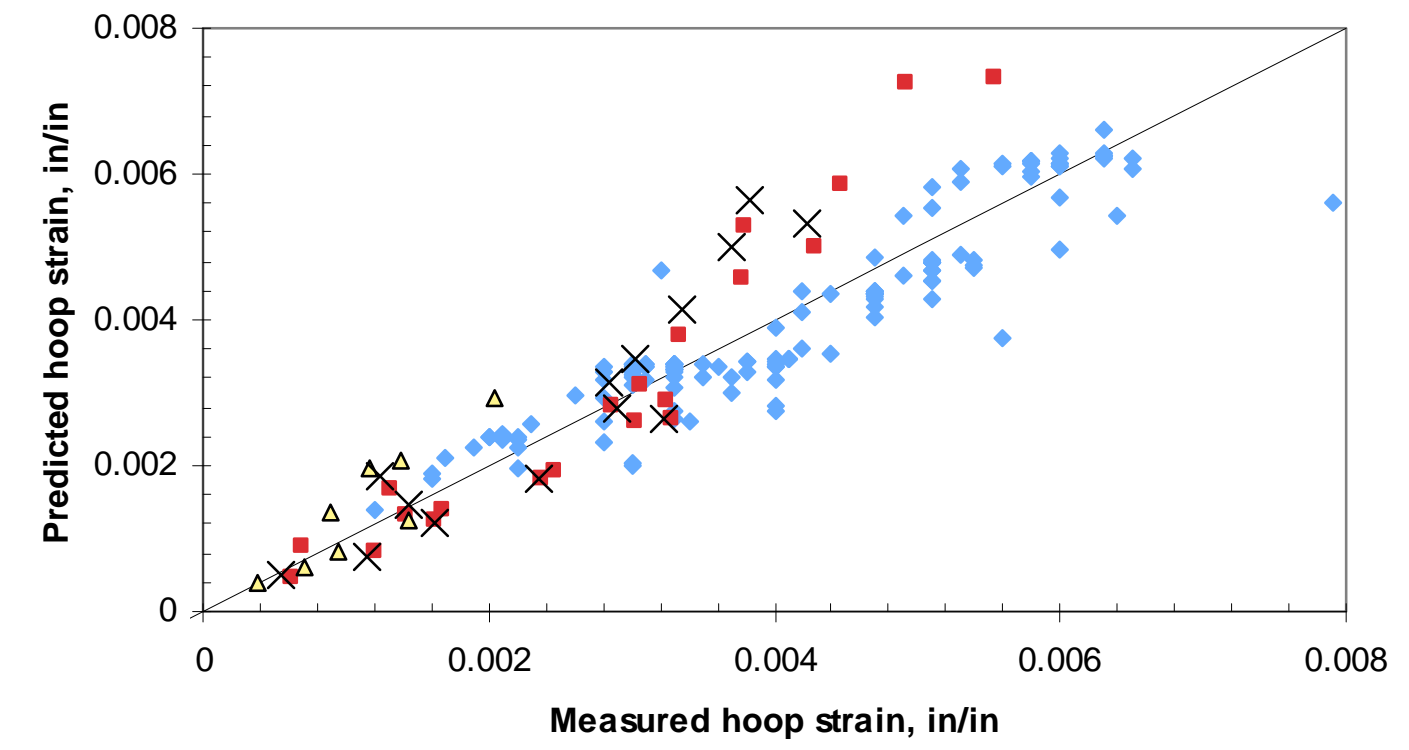

- Franklin - Soniak $350^{\circ} \mathrm{C} \Delta$ Soniak $320^{\circ} \mathrm{C} \times$ Gilbon _ Predicted=Measured

Figure 3.8-2 Predicted vs. measured hoop strain for RXA Zircaloy model.

\subsubsection{Applicability and Uncertainty}

The subroutine CREPR is applicable over the range of conditions for which the data was collected. The database spans a temperature range of 570 to $625 \mathrm{~K}$, an effective stress range of 40 to $130 \mathrm{MPa}$, and a fast neutron flux range of $1 \times 10^{17}$ to $2 \times 10^{18} \mathrm{n} / \mathrm{m}^{2}$-s. This covers the nominal 
operating conditions for LWRs. The relative standard error for the predictions is 14.5 percent for SRA and 21.6 percent for RXA cladding. The uncertainty for predicting cladding creep in a fuel rod will most likely be greater than these values because there may be greater uncertainty in the estimate of fast neutron flux and cladding temperature than those from the creep data used to determine the creep model uncertainties.

\subsubsection{References}

Limbäck, M., and T. Andersson. 1996. “A Model for Analysis of the Effect of Final Annealing on the In- and Out-of-Reactor Creep Behavior of Zircaloy Cladding," Zirconium in the Nuclear Industry: Eleventh International Symposium, ASTM STP 1295, E.R. Bradley and G.P. Sabol, eds., American Society for Testing and Materials, West Conshohocken, PA, pp. 448-468.

Matsuo, Y. 1987. “Thermal Creep of Zircaloy-4 Cladding under Internal Pressure,” Journal of Nuclear Science and Technology, 24(2):111-119.

Franklin, D.G., G.E. Lucas, A.L. Bement. 1983. "Creep of Zirconium Alloys in Nuclear Reactors,” ASTM STP 815, American Society for Testing and Materials, West Conshohocken, PA.

Gilbon, D., A. Soniak, S. Doriot, and J.P. Mardon. 2000. “Irradiation Creep and Growth Behavior, and Microstructural Evolution of Advanced Zr-Base Alloys," Zirconium in the Nuclear Industry: Twelfth International Symposium, ASTM-STP-1354, G.P. Sabol and G.D. Moan, eds., American Society of Testing and Materials, West Conshohocken, PA, pp. 51-73.

Soniak, A., N. L’Hullier, J-P. Mardon, V. Rebeyrolle, P. Boffioux, C. Bernaudat. 2002. "Irradiation Creep Behavior of Zr-Base Alloys," Zirconium in the Nuclear Industry: Thirteenth International Symposium, ASTM STP 1423, G.D. Moan and P. Rudling, eds., ASTM International, West Conshohocken, PA, pp.837-862.

Sabol, G.P., Comstock, R.J., Weiner, R.A., Larouere, P., Stanutz, R.N. 1994. "In-Reactor Corrosion Performance of ZIRLO ${ }^{\mathrm{TM}}$ and Zircaloy-4," Zirconium in the Nuclear Industry: Tenth International Symposium, ASTM STP 1245, A.M. Garde and E.R. Bradley, eds. American Society for Testing and Materials, Philadelphia, PA, pp. 724-744.

\subsection{Cladding Meyer Hardness (CMHARD)}

The subroutine CMHARD calculates Meyer hardness as a function of cladding temperature. Hardness is one of the parameters required for calculating fuel-to-cladding contact conductance. As the contact pressure between the two surfaces increases, the points of contact enlarge due to localized plastic deformation and the solid-to-solid thermal conductance is improved. The Meyer hardness is used by Ross and Stoute (1962) in their heat transfer correlation as an indication of the hardness of resistance to deformation of the softer (Zircaloy) material.

The same CMHARD subroutine is used in the MATPRO, FRAPCON-3.4, and FRAPTRAN 1.4 codes. However, FRAPTRAN 1.4 includes additional coding that ensures that the minimum hardness returned is $1.94 \times 10^{8} \mathrm{~N} / \mathrm{m}^{2}$ (the highest temperature data point) and includes provisions for $\mathrm{ZrNb}-1$. 


\subsubsection{Model Development and Comparisons}

In MATPRO, the Meyer hardness number is a measure of indentation hardness and is defined in conjunction with Meyer's law, which is presented in Equation 3.9-1.

where

$$
\mathrm{L}=\operatorname{ad}^{\mathrm{n}}
$$

$\mathrm{L} \quad=\quad$ load

$\mathrm{d}=\quad$ the diameter of impression at the surface of a specimen in a static ball test

$\mathrm{n}=$ the Meyer work hardening coefficient

$\mathrm{a}=\mathrm{a}$ material constant

The Meyer hardness number (MH) is defined as $4 \mathrm{~L} / \pi \mathrm{d}^{2}$. Other hardness numbers are available (Brinell, Rockwell, etc.), and conversion from one to another is possible. However, the routine CMHARD was created to provide information required by the Ross and Stoute gap conductance model that includes a dependence on Meyer hardness.

Meyer hardness numbers for temperatures from 298 to 877K were taken from Peggs and Godin (1975). A regression analysis of the reciprocal of the Meyer hardness values versus the log of temperature was used to obtain the analytical expression used in CMHARD. The correlation used is given by Equation 3.9-2.

$$
\mathrm{MH}=\exp \left\{2.6034 \times 10^{1}+\mathrm{T}\left\{-2.6394 \times 10^{-2}+\mathrm{T}\left[4.3502 \times 10^{-5}+\mathrm{T}\left(2.5621 \times 10^{-8}\right)\right]\right\}\right\}
$$

where

$\begin{array}{lll}\mathrm{MH} & = & \text { Meyer hardness }\left(\mathrm{N} / \mathrm{m}^{2}\right) \\ \mathrm{T} & = & \text { temperature }(\mathrm{K})\end{array}$

The Meyer hardness decreased rapidly with increasing temperature, beginning at $2 \times 10^{9} \mathrm{MPa}$ at room temperature and decreasing to $2 \times 10^{8} \mathrm{MPa}$ at $875 \mathrm{~K}$. The hardness is presumed to continue its rapid rate of decrease at temperatures above $875 \mathrm{~K}$. The minimum Meyer hardness number of Zircaloy cladding is $1.0 \times 10^{5} \mathrm{~N} / \mathrm{m}^{2}$.

A comparison between the CMHARD correlation and the data from which it was derived is presented in Figure 3.9-1. 


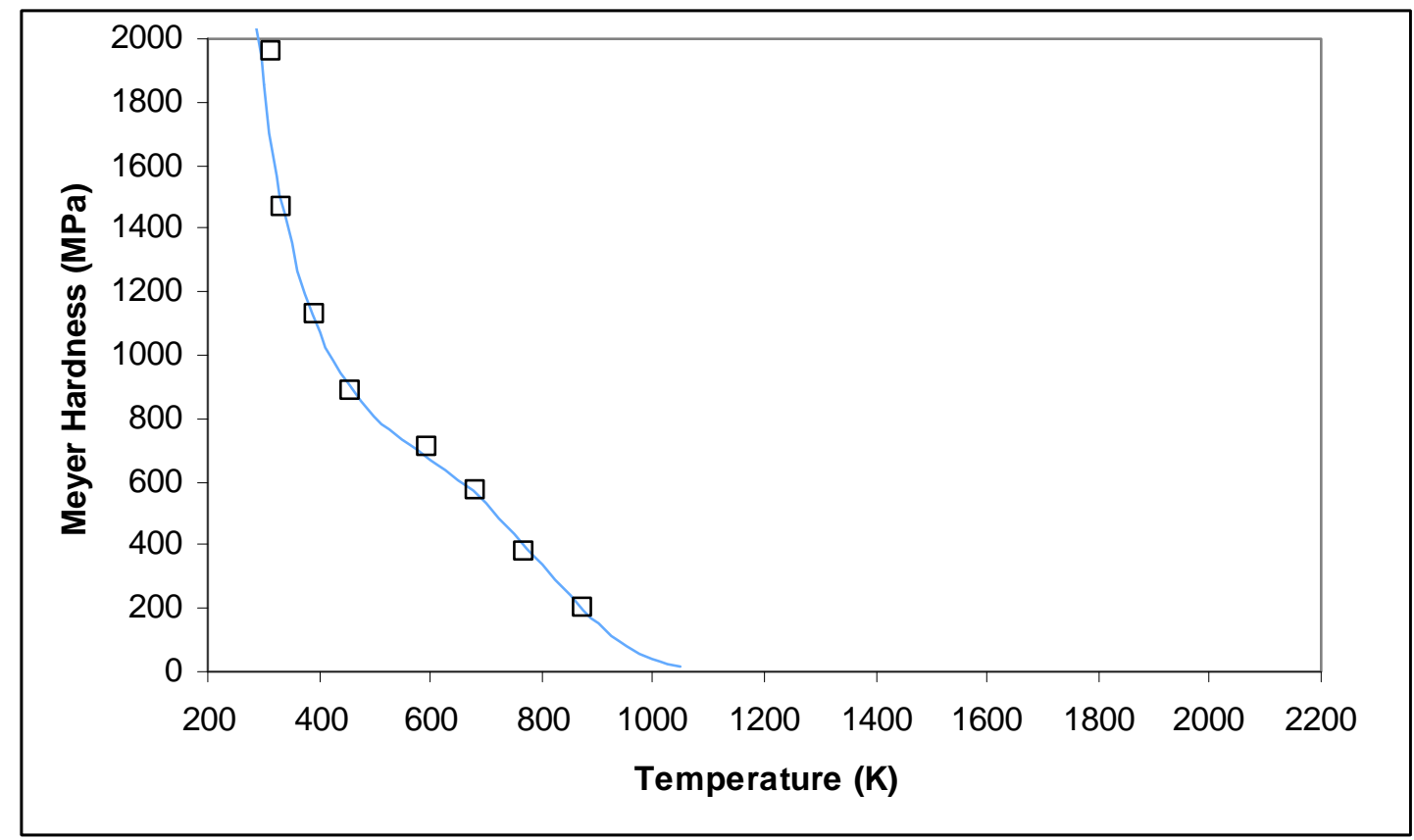

Figure 3.9-1 CMHARD correlation as a function of temperature with data from Peggs and Godin.

\subsubsection{Applicability and Uncertainty}

The subroutine CMHARD is applicable for temperatures up to 875K. An estimate of the uncertainty has not been established for this correlation due to the limited data.

\subsubsection{References}

Ross, A.M., and R.L. Stoute. 1962. Heat Transfer Coefficient Between $\mathrm{UO}_{2}$ and Zircaloy-2, AECL-1552, Atomic Energy of Canada, Ltd., Chalk River, Ontario.

Peggs, I.D., and D.P. Godin. 1975. “The Yield Strength-Hot Hardness Relationship of Zircaloy4,” Journal of Nuclear Materials, 57:246-248. 


\subsection{Gas Material Properties}

This section describes material property correlations for gap gases. The subroutine used to determine the material correlation in either the FRAPCON-3/FRAPTRAN codes is given in parentheses in the heading of each subsection.

\subsection{Gas Conductivity (GTHCON)}

The subroutine GTHCON calculates the gas thermal conductivity as a function of temperature and gas fraction for seven gases: helium (He), argon (Ar), krypton (Kr), xenon (Xe), hydrogen $(\mathrm{H})$, nitrogen $(\mathrm{N})$, and water vapor (steam). The MATPRO, FRAPCON-3.4, and FRAPTRAN 1.4 codes use similar correlations to determine the gas thermal conductivity. However, FRAPCON-3.4 and FRAPTRAN 1.4 use updated fitting parameters to better estimate gas thermal conductivity at higher temperatures.

\subsubsection{Model Development and Comparisons}

The heat conductance of gas-filled gaps or pores is dependent on the thermal conductivity of the gas mixture when the dimensions of the gas-filled regions are large compared to the mean distance between gas molecule collisions (mean free path of the gas molecules). This section presents data and correlations for the thermal conductivities of the gases of interest in fuel rod analysis.

The correlations used for the gases of interest are all of the form presented in Equation 4.1-1.

where

$$
\mathrm{K}=\mathrm{AT}^{\mathrm{B}}
$$

$\mathrm{K}=$ thermal conductivity $(\mathrm{W} / \mathrm{m}-\mathrm{K})$

$\mathrm{T}=$ gas temperature $(\mathrm{K})$

The constants A and B are fitting parameters. The values for A and B used in MATPRO for each gas are given in Table 4.1-1 and the values used in FRAPCON-3.4 and FRAPTRAN 1.4 (FRAP) are given in Table 4.1-2.

Table 4.1-1 Constants used in gas thermal conductivity correlations in MATPRO.

\begin{tabular}{|c|c|c|}
\hline Gas & A & B \\
\hline $\mathrm{He}$ & $2.639 \times 10^{-3}$ & 0.7085 \\
\hline $\mathrm{Ar}$ & $2.986 \times 10^{-4}$ & 0.7224 \\
\hline $\mathrm{Kr}$ & $8.247 \times 10^{-5}$ & 0.8363 \\
\hline $\mathrm{Xe}$ & $4.351 \times 10^{-5}$ & 0.8616 \\
\hline $\mathrm{H}_{2}$ & $1.097 \times 10^{-3}$ & 0.8785 \\
\hline $\mathrm{N}_{2}$ & $5.314 \times 10^{-4}$ & 0.6898 \\
\hline
\end{tabular}


Table 4.1-2 Constants used in gas thermal conductivity correlations in FRAP.

\begin{tabular}{|c|c|c|}
\hline Gas & A & B \\
\hline $\mathrm{He}$ & $2.531 \times 10^{-3}$ & 0.7146 \\
\hline $\mathrm{Ar}$ & $4.092 \times 10^{-4}$ & 0.6748 \\
\hline $\mathrm{Kr}$ & $1.966 \times 10^{-4}$ & 0.7006 \\
\hline $\mathrm{Xe}$ & $9.825 \times 10^{-5}$ & 0.7334 \\
\hline $\mathrm{H}_{2}$ & $1.349 \times 10^{-3}$ & 0.8408 \\
\hline $\mathrm{N}_{2}$ & $2.984 \times 10^{-4}$ & 0.7799 \\
\hline
\end{tabular}

The MATPRO steam correlation is also used in FRAPCON-3.4/FRAPTRAN 1.4 codes. For temperatures less than or equal to $973.15 \mathrm{~K}$, the correlation presented in Equation 4.1-2 is used. For temperatures greater than $973.15 \mathrm{~K}$, the correlation presented in Equation 4.1-3 is used to determine the thermal conductivity of steam. Temperature, $\mathrm{T}$, is in kelvin and pressure, $\mathrm{P}$, is in pascals.

$$
\begin{gathered}
\mathrm{k}=4.44 \times 10^{-6} * \mathrm{~T}^{1.45}+9.45 \times 10^{-5} *\left(2.1668 \times 10^{-9} * \mathrm{P} / \mathrm{T}\right)^{1.3} \\
\mathrm{k}=\mathrm{P} / \mathrm{T} *\left(-2.8516 \times 10^{-8}+9.424 \times 10^{-10} * \mathrm{~T}-6.005 \times 10^{-14} * \mathrm{~T}^{2}\right)+\ldots \\
1.009 * \mathrm{P}^{2} / \mathrm{T}^{2} /(\mathrm{T}-273.15)^{4.2}+\left[17.6 \times 10^{-4}+5.87 \times 10^{-5} *(\mathrm{~T}-273.15)+\ldots\right. \\
\left.1.08 \times 10^{-7} *(\mathrm{~T}-273.15)^{2}-4.51 \times 10^{-11} *(\mathrm{~T}-273.15)^{3}\right]
\end{gathered}
$$

The thermal conductivity of gas mixtures is calculated with Equation 4.1-4.

$$
\mathrm{k}_{\text {mix }}=\sum_{\mathrm{i}}^{\mathrm{n}}\left(\frac{\mathrm{k}_{\mathrm{i}} \mathrm{x}_{\mathrm{i}}}{\mathrm{x}_{\mathrm{i}}+\sum_{\mathrm{j}=1}^{\mathrm{n}}\left(1-\delta_{\mathrm{ij}}\right) \Psi_{\mathrm{ij}} \mathrm{x}_{\mathrm{j}}}\right)
$$

where $\Psi_{\mathrm{ij}}$ is given in Equation 4.1-5

$$
\Psi_{\mathrm{ij}}=\phi_{\mathrm{ij}}\left(1+2.41 \frac{\left(\mathrm{M}_{\mathrm{i}}-\mathrm{M}_{\mathrm{j}}\right)\left(\mathrm{M}_{\mathrm{i}}-0.142 \mathrm{M}_{\mathrm{j}}\right)}{\left(\mathrm{M}_{\mathrm{i}}+\mathrm{M}_{\mathrm{j}}\right)^{2}}\right)
$$

and $\phi_{\mathrm{ij}}$ is given in Equation 4.1-6

and

$$
\phi_{i j}=\frac{\left[1+\left(\frac{k_{i}}{k_{j}}\right)^{1 / 2}\left(\frac{M_{i}}{M_{j}}\right)^{1 / 4}\right]^{2}}{2^{3 / 2}\left(1+\frac{M_{i}}{M_{j}}\right)^{1 / 2}}
$$

\footnotetext{
$\delta_{\mathrm{ij}} \quad=\quad$ Kronecker delta $=1$ for $\mathrm{i}=\mathrm{j}, 0$ otherwise (unitless)

$\mathrm{n}=$ number of components in mixture (unitless)

$\mathrm{M}_{\mathrm{i}} \quad=\quad$ molecular weight of component $\mathrm{i}(\mathrm{kg})$
} 


$$
\begin{array}{lll}
\mathrm{x}_{\mathrm{i}} & = & \text { mole fraction of component } \mathrm{i} \text { (unitless) } \\
\mathrm{k}_{\mathrm{i}} & = & \text { thermal conductivity of the component } \mathrm{i}(\mathrm{W} / \mathrm{m}-\mathrm{K})
\end{array}
$$

Model-to-data comparisons for the gas conductivity models are presented in Figures 4.1-1 through 4.1-7.

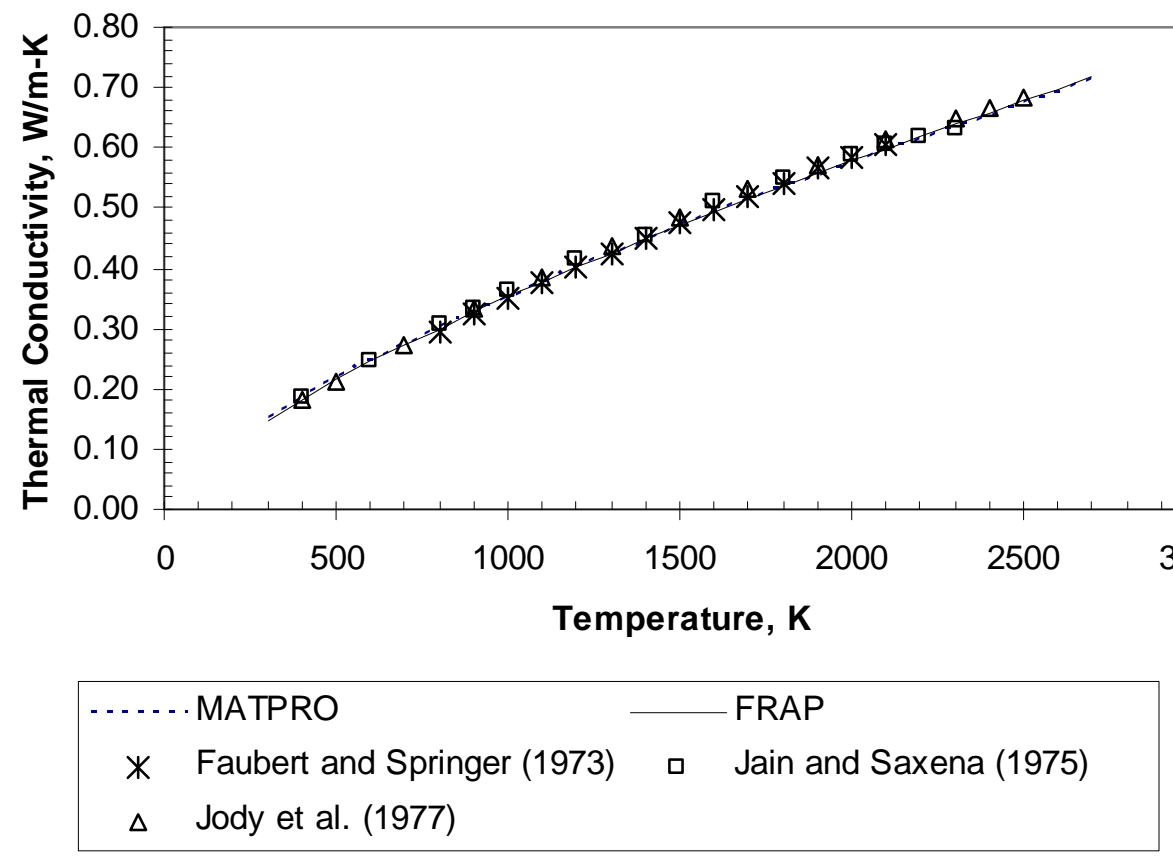

Figure 4.1-1 Model-to-data comparison for He thermal conductivity. 


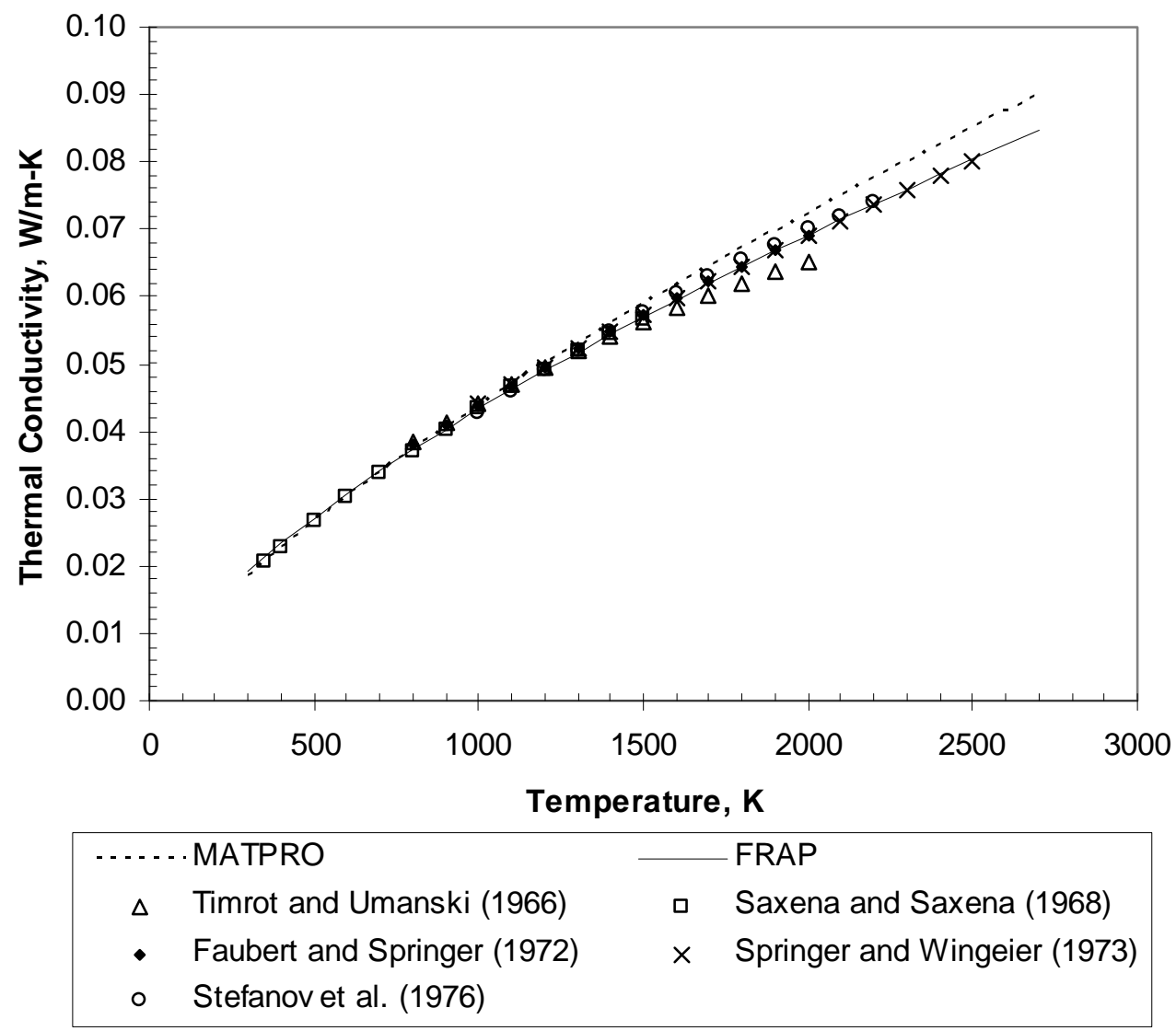

Figure 4.1-2 Model-to-data comparison for Ar thermal conductivity. 


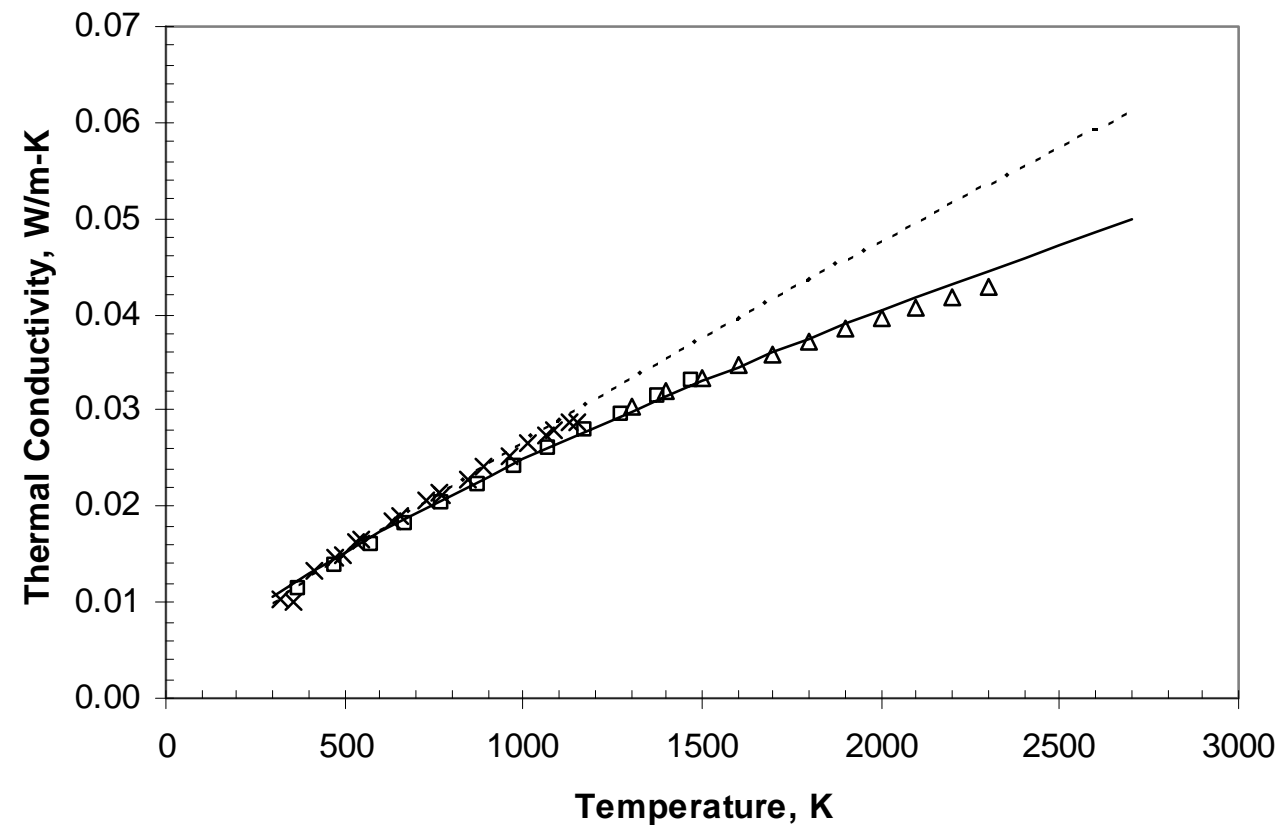

\begin{tabular}{|c|c|c|c|}
\hline & MATPRO & & FRAP \\
\hline 口 & Saxena and Saxena (1969) & $\Delta$ & Stefanov et al. (1976) \\
\hline$x$ & Vargaftik and Yakush (1971) & & \\
\hline
\end{tabular}

Figure 4.1-3 Model-to-data comparison for Kr thermal conductivity. 


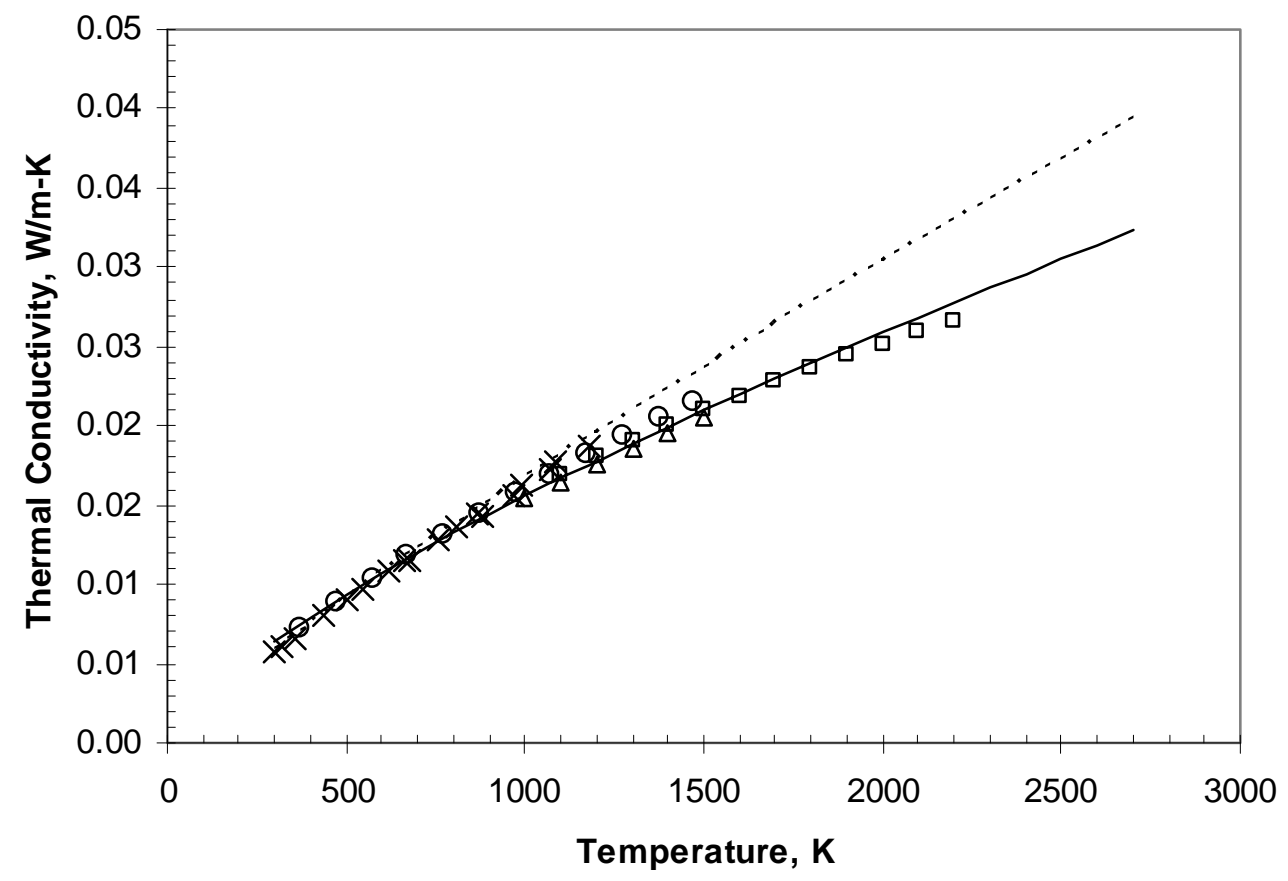

\begin{tabular}{|c|c|c|c|}
\hline - & MATPRO & 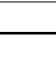 & FRAP \\
\hline$\square$ & Stefanov (1976) & $\Delta$ & Springer and Wigneier (1973) \\
\hline 0 & Saxena and Saxena (1969) & $x$ & Vargaftik and Yakush (1971) \\
\hline
\end{tabular}

Figure 4.1-4 Model-to-data comparison for Xe thermal conductivity.

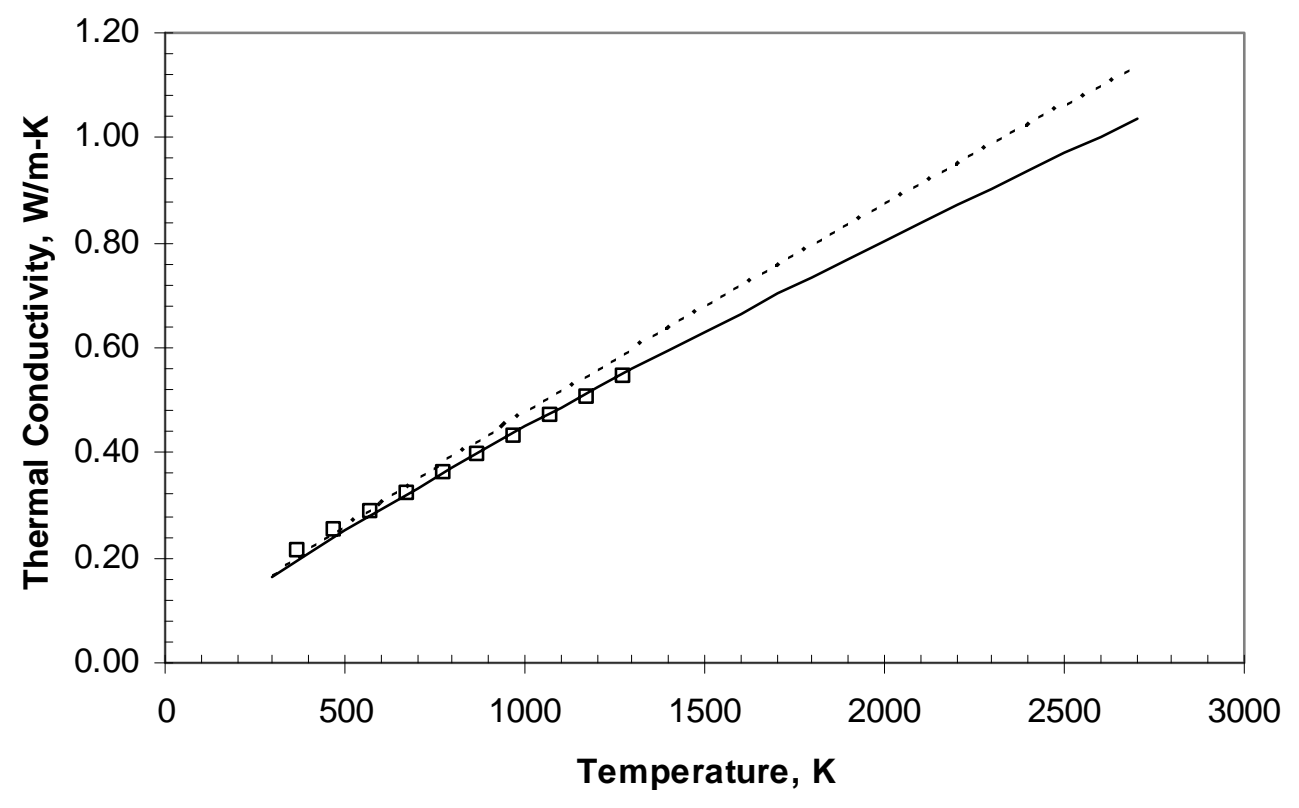

.......MATPRO — FRAP 口 Saxena and Saxena (1970)

Figure 4.1-5 Model-to-data comparison for $\mathrm{H}_{2}$ thermal conductivity. 


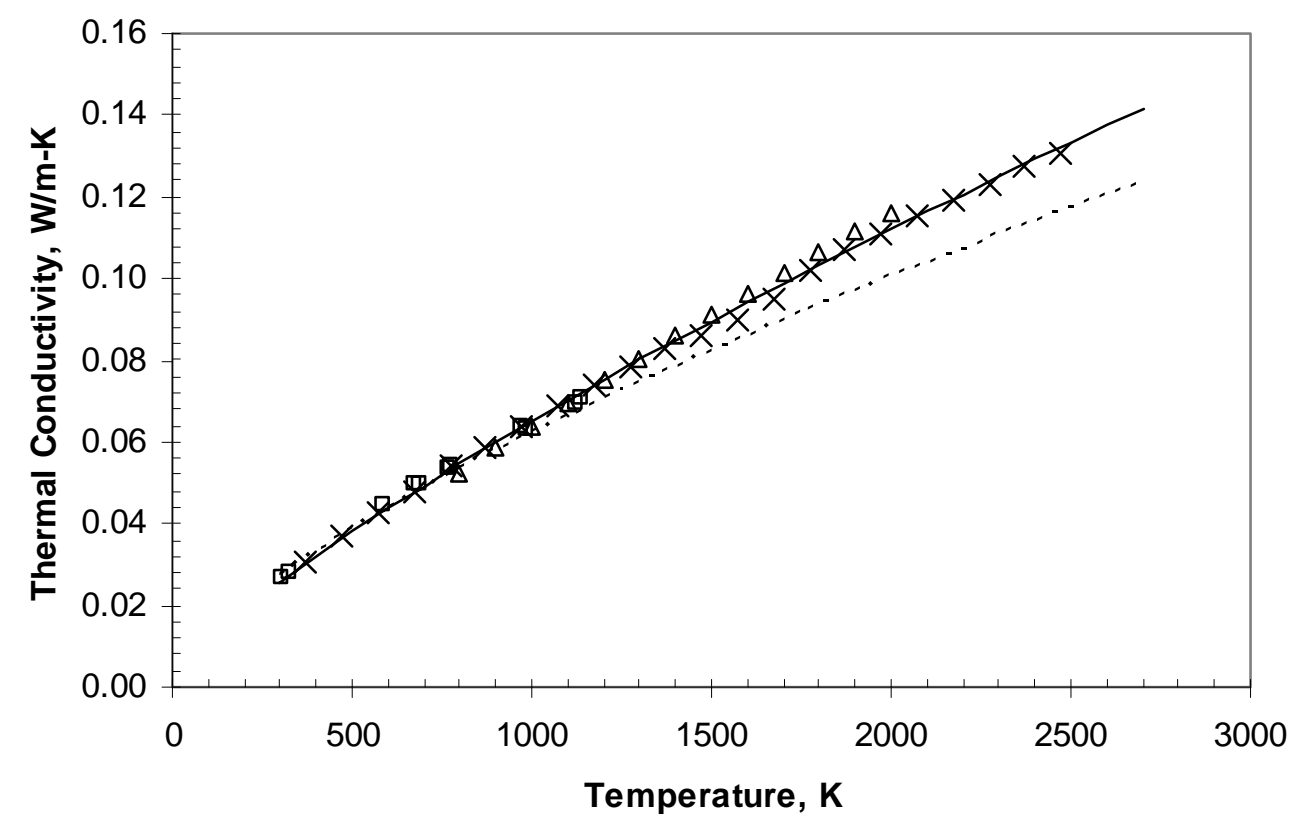

\begin{tabular}{|c|c|c|c|}
\hline$\cdots$ & MATPRO & & -FRAP \\
\hline$\square$ & Vargaftik and Zimina (1964) & $\Delta$ & Faubert and Springer (1972) \\
\hline$x$ & Chen and Saxena (1973) & & \\
\hline
\end{tabular}

Figure 4.1-6 Model-to-data comparison for $\mathrm{N}_{2}$ thermal conductivity.

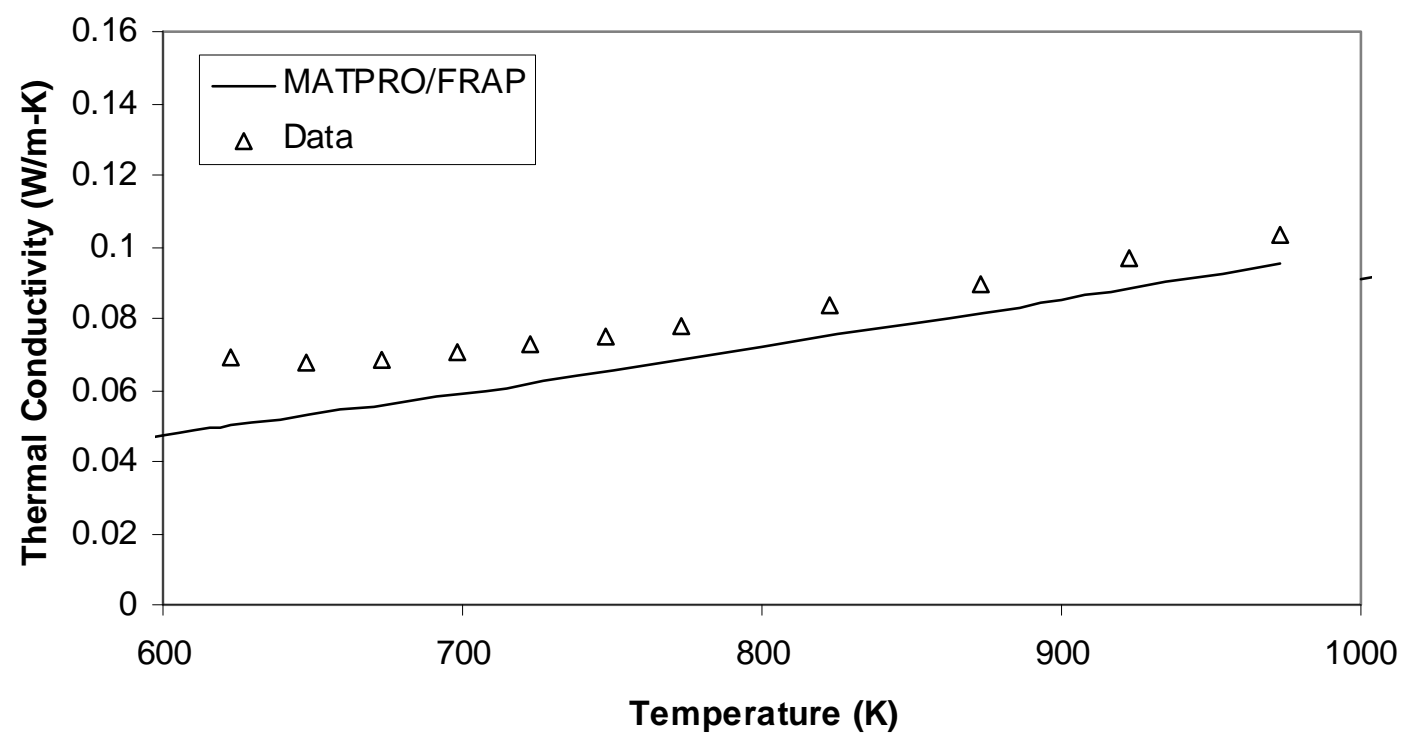

Figure 4.1-7 Model-to-data comparison for steam thermal conductivity.

\subsubsection{Applicability and Uncertainty}

The gas thermal conductivity correlations for used in GTHCON are applicable over the temperature range for which data was collected. The standard error for each correlation is given in Table 4.1-3. 
Table 4.1-3 Standard deviation of gas thermal conductivity correlations.

\begin{tabular}{|c|c|}
\hline Gas & Standard Deviation \\
\hline $\mathrm{He}$ & $8.99 \times 10^{-3}$ \\
\hline $\mathrm{Ar}$ & $9.66 \times 10^{-4}$ \\
\hline $\mathrm{Kr}$ & $8.86 \times 10^{-4}$ \\
\hline $\mathrm{Xe}$ & $5.34 \times 10^{-4}$ \\
\hline $\mathrm{H}_{2}$ & $1.67 \times 10^{-2}$ \\
\hline $\mathrm{N}_{2}$ & $1.97 \times 10^{-4}$ \\
\hline Steam & $1.69 \times 10^{-3}$ \\
\hline
\end{tabular}

\subsubsection{References}

Faubert, F.M., and G.S. Springer. 1973. "Measurement of the Thermal Conductivity of Helium up to $2100^{\circ} \mathrm{K}$ by the Column Method,” Journal of Chemical Physics, 58(10):4080-4083.

Jain, P.C., and S.C. Saxena. 1975. "Transport Properties of Helium in the Temperature Range 400-2300K,” Chemical Physics Letters, 36(4):489-491.

Jody, B.J., S.C. Saxena, V.P.S. Nain, and R.A. Aziz. 1977. "Thermal Conductivity of Helium: A Problem for the Repulsive Wall of the Interatomic Potential,” Chemical Physics, 22:53-58.

Timrot, D.L., and A.S. Umanskii. 1966. “Thermal Conductivity of Hydrogen and Argon,” High Temperature, 3:285-287.

Saxena, V.K., and S.C. Saxena. 1968b. "Measurement of the Thermal Conductivity of Argon Using Hot-Wire Type Thermal Diffusion Columns,” Chemical Physics Letters, 2(1):44-46.

Faubert, F.M., and G.S. Springer. 1972. "Measurement of the Thermal Conductivity of Argon, Krypton, and Nitrogen in the Range 800-2000 K," Journal of Chemical Physics, 57(6):23332340.

Springer, G.S., and E.W. Wingeier. 1973. "Thermal Conductivity of Neon, Argon, and Xenon at High Temperatures,” Journal of Chemical Physics, 59(5):2747-2750.

Stefanov, B., L. Zarkova, and D. Oliver. 1976. "Measurement of the Thermal-Conductivity Coefficient of Gases and Vapors up to $2500^{\circ} \mathrm{K}$,” High Temperature, 14(1):48-57.

Saxena, V.K., and S.C. Saxena. 1969. "Thermal Conductivity of Krypton and Xenon in the Temperature Range 350-1500K,” Journal of Chemical Physics, 51(8):3361-3368.

Vargaftik, N.B., and L.V. Yakush. 1971. "Measurement of the Thermal Conductivities of Neon, Krypton, and Xenon over a Wide Range of Temperatures,” Journal of Engineering Physics, 21(3):1156-1161.

Saxena, S.C., and V.K. Saxena. 1970. "Thermal Conductivity Data for Hydrogen and Deuterium in the Range $100-1100^{\circ} \mathrm{C}$," Journal of Physics A, 3(3):309-320.

Vargaftik, N.B., and N.Kh. Zimina. 1964. "Thermal Conductivity of Nitrogen at High Temperatures,” High Temperature, 2(6):782-790. 
Chen, S.H.P., and S.C. Saxena. 1973. "Experimental Determination of Thermal Conductivity of Nitrogen in the Temperature Range 100-2200 C," High Temperature Science, 5(3):206-233. 
Appendix A

\section{Subroutine Source Codes}




\section{Appendix A: Subroutine Source Codes}

\section{A.1 Uranium Dioxide/Mixed Oxide Properties}

\section{A.1.1 Fuel Melting Temperature (PHYPRP)}

\section{FRAPCON-3.4: PHYPRP}

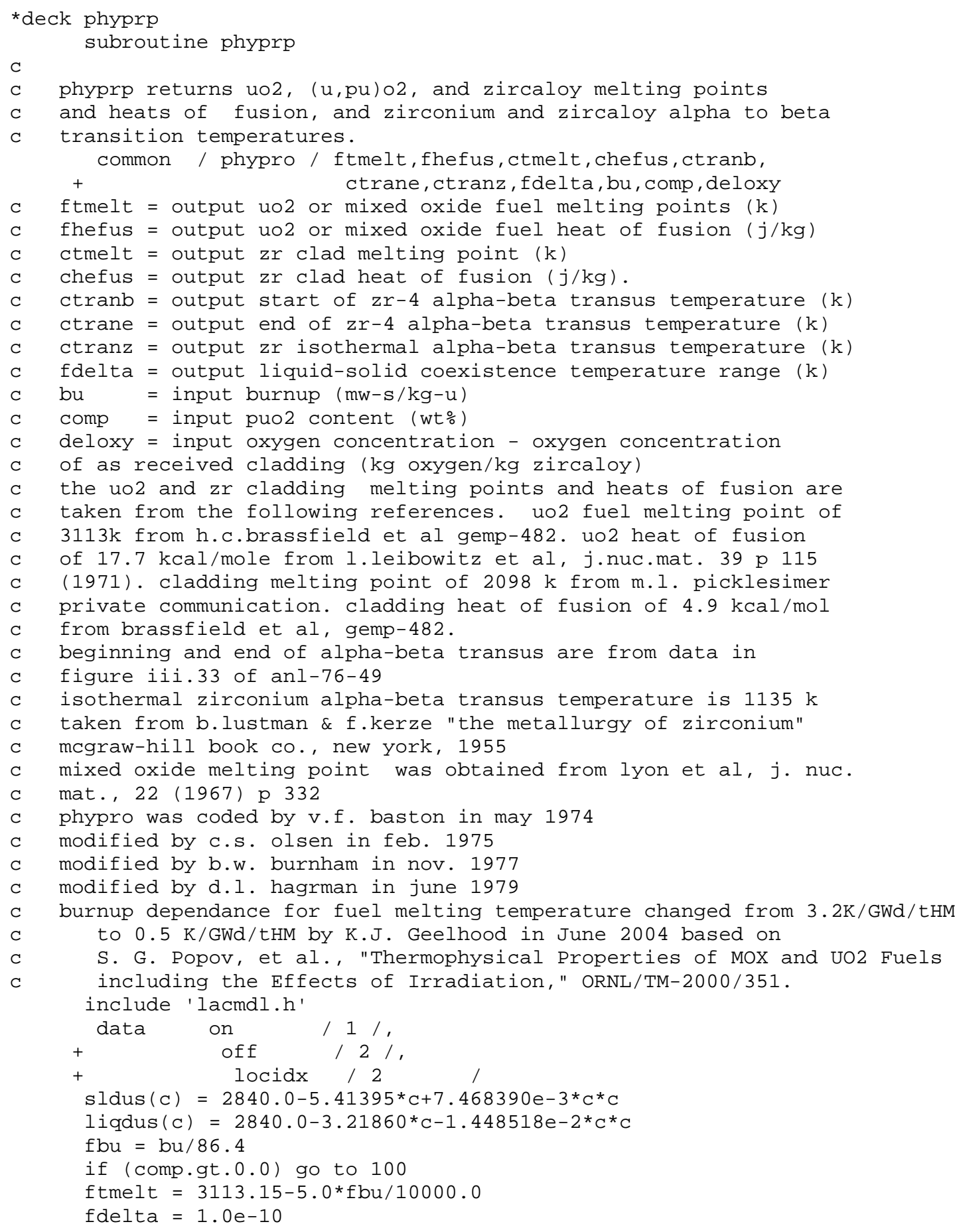


go to 110

100

$\mathrm{c} 1=\mathrm{comp}$

ftmelt $=$ sldus $(\mathrm{c} 1)+273.15-5.0 * \mathrm{fbu} / 10000.0$

fdelta $=$ liqdus $(\mathrm{c} 1)-\operatorname{sldus}(\mathrm{c} 1)-5.0 * \mathrm{fbu} / 10000 . \odot$

110

fhefus $=27.4 \mathrm{e}+4$

ctmelt $=2098.15$

chefus $=22.5 \mathrm{e}+04$

wfox $=$ deloxy+0.0012

ctranb $=1094 .+$ wfox $*(-1.289 e+03+w f o x * 7.914 e+05)$

if (wfox.lt.0.025) go to 120

ctranb $=1556.4+3.8281 \mathrm{e}+04 *($ wfox -0.025$)$

120 ctrane $=392.46 *\left(\left(100 .{ }^{*}\right.\right.$ deloxy +0.1242807$\left.) * * 2+3.1417\right)$

if (deloxy.lt.4.7308937e-03) go to 130

ctrane $=\left(100 .{ }^{*}\right.$ deloxy +0.12$) * 491.157+1081.7413$

130

continue

ctranz $=1135.15$

return

end

\section{FRAPTRAN 1.4: PHYPRP}

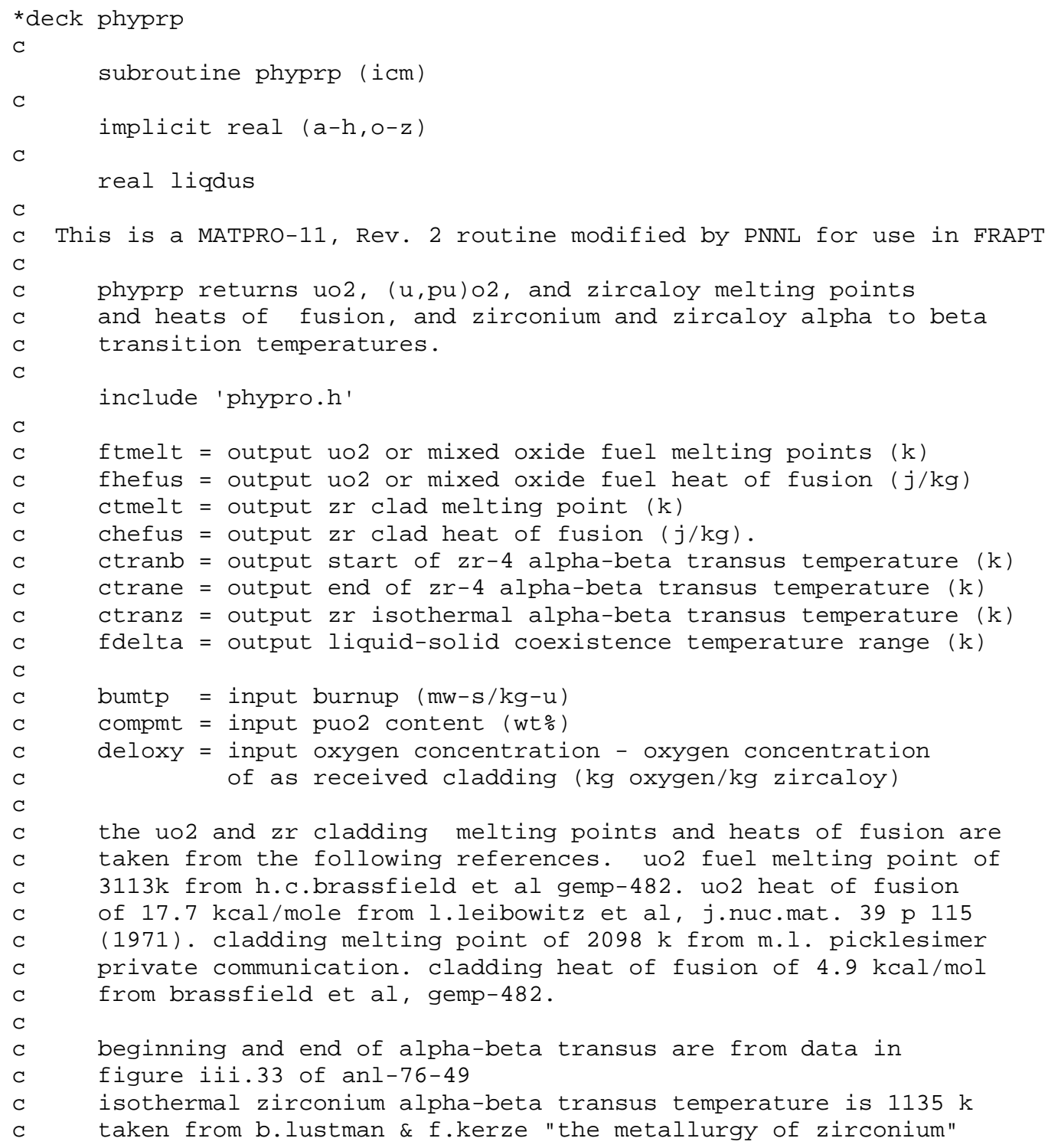




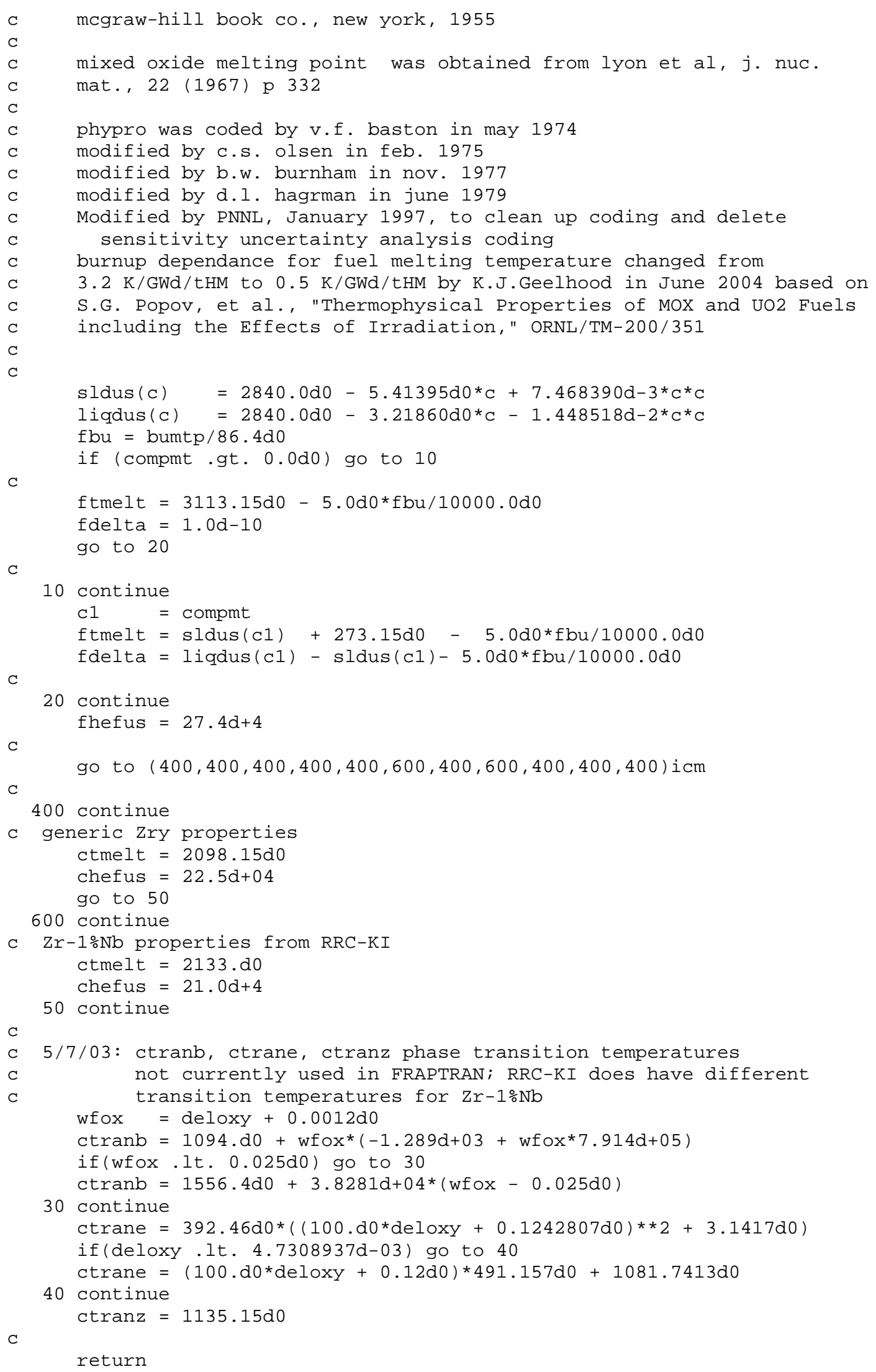




\section{A.1.2 Fuel Specific Heat Capacity (FCP) and Fuel Enthalpy (FENTHL)}

\section{FRAPCON-3.4: FCP}

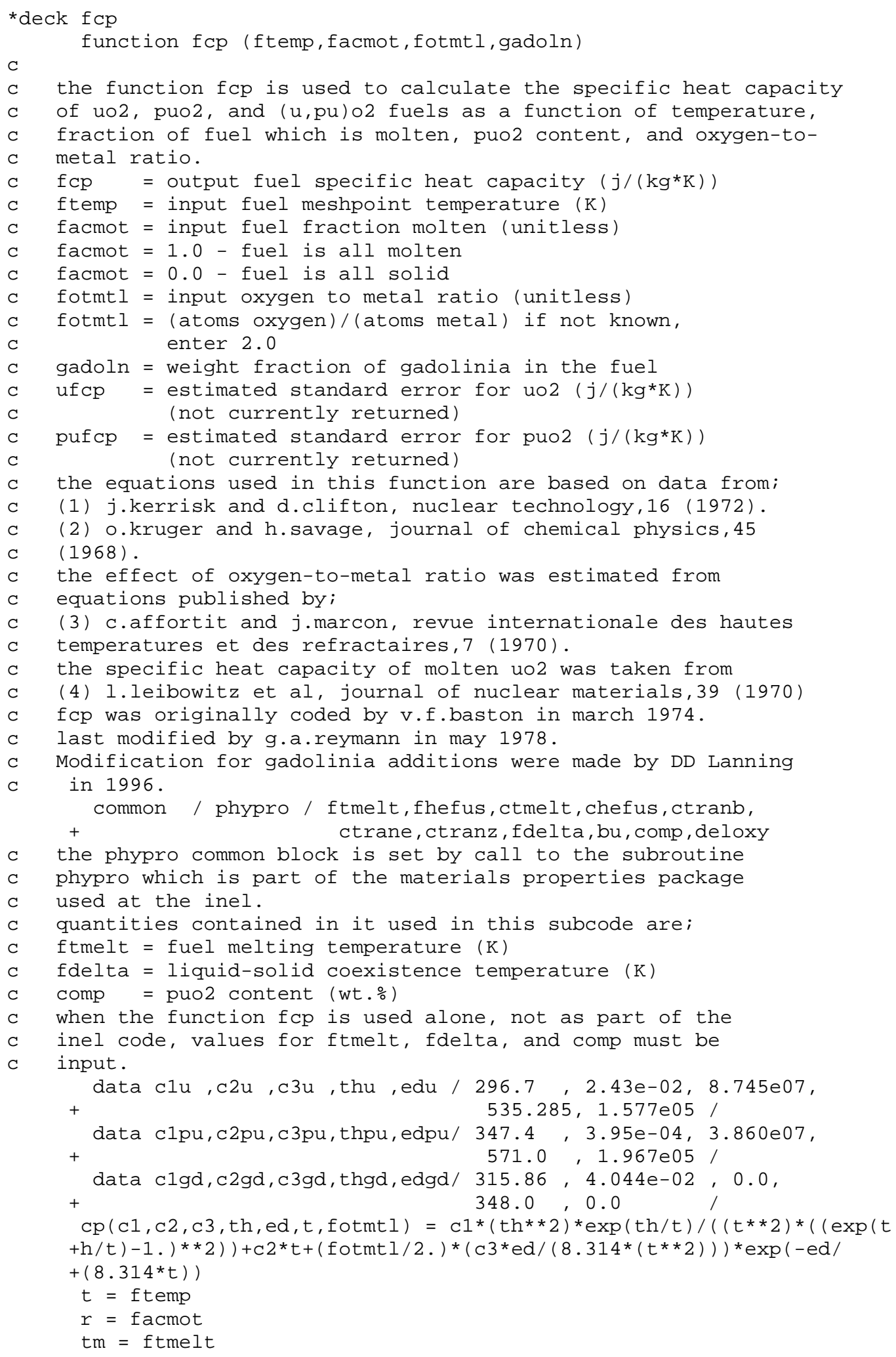


fcomp $=$ comp $/ 100.0$

fcpmol $=503.0$

c fcpmol = specific heat capacity of molten fuel $\left(j /\left(\mathrm{kg}^{*} \mathrm{~K}\right)\right)$

if (t.gt. (tm+fdelta)) go to 100

$f c p=c p(c 1 u, c 2 u, c 3 u, t h u, e d u, t, f o t m t l) *(1.0-f c o m p)+c p(c 1 p u, c 2 p u, c 3$

+ pu, thpu, edpu, $t$, fotmtl)* fcomp

$f c p=f c p *(1.0-$ gadoln $)+$ gadoln*cp $(c 1 g d, c 2 g d, c 3 g d$, thgd, edgd, $t$,

+ fotmtl)

if (t.lt.(tm-0.1)) go to 110

$f c p=(1 . \Theta-r) * f c p+r * f c p m o l$

go to 110

$100 \quad \mathrm{fcp}=\mathrm{fcpmol}$

ufcp $=2.0$

pufcp $=5.6$

110

continue

return

end

\section{FRAPTRAN 1.4: FCP}

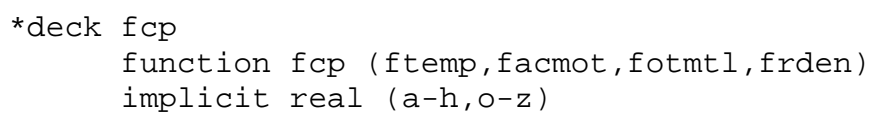

C

C

C

C

C

C

C

C

$\mathrm{C}$

C

$\mathrm{C}$

C

C

C

C

C

C

C

C

C

C

C

C

C

C

C

C

C

C

C

C

C

C

This is a MATPRO-11, Rev. 2 routine modified by PNNL for use in FRAPT

the function fcp is used to calculate the specific heat capacity of uo2, puo2, and ( $u, p u) 02$ fuels as a function of temperature, fraction of fuel which is molten, puo2 content, and oxygen-tometal ratio.

fcp = output fuel specific heat capacity $\left(j /\left(k g^{*} k\right)\right)$

ftemp = input fuel meshpoint temperature $(\mathrm{k})$

facmot $=$ input fuel fraction molten (unitless)

facmot $=1.0$ - fuel is all molten

facmot $=0.0-$ fuel is all solid

fotmtl = input oxygen to metal ratio (unitless)

fotmtl = (atoms oxygen)/(atoms metal) if not known, enter 2.0

ufcp = estimated standard error for uo2 $\left(j /\left(\mathrm{kg}^{*} \mathrm{k}\right)\right)$

(not currently returned)

pufcp = estimated standard error for puo2 $\left(j /\left(\mathrm{kg}^{*} \mathrm{k}\right)\right)$ (not currently returned)

the equations used in this function are based on data from;

(1) j.kerrisk and d.clifton, nuclear technology, 16 (1972).

(2) o.kruger and h.savage, journal of chemical physics, 45 (1968).

the effect of oxygen-to-metal ratio was estimated from equations published by;

(3) c.affortit and j.marcon, revue internationale des hautes temperatures et des refractaires, 7 (1970).

the specific heat capacity of molten uo2 was taken from

(4) 1.leibowitz et al, journal of nuclear materials, 39 (1970)

fcp was originally coded by v.f.baston in march 1974 .

last modified by g.a.reymann in may 1978 .

Modified by PNNL, January 1997, to clean up coding and delete lic analysis and sensitivity uncertainty coding 


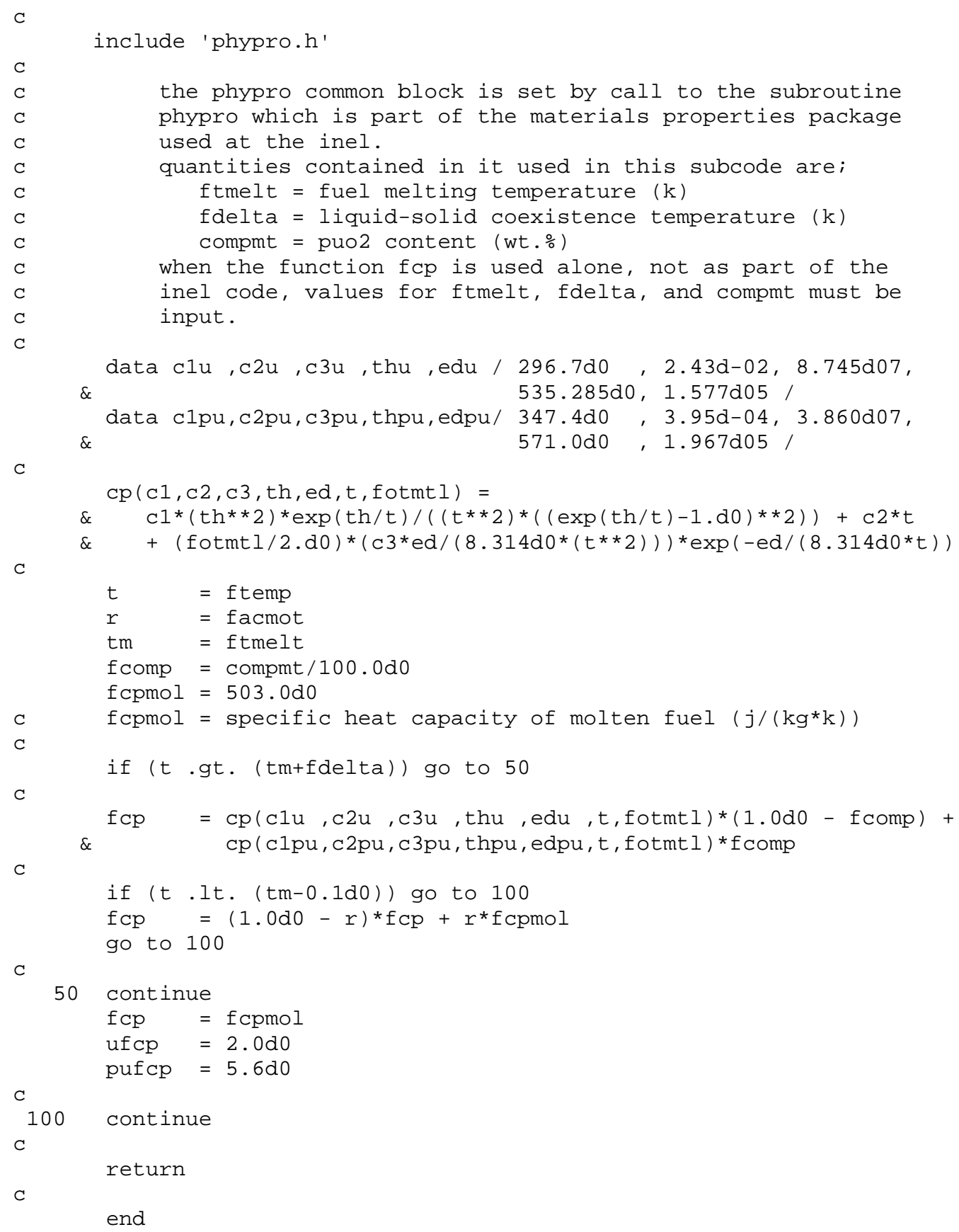

\section{FRAPCON-3.4: FENTHL}

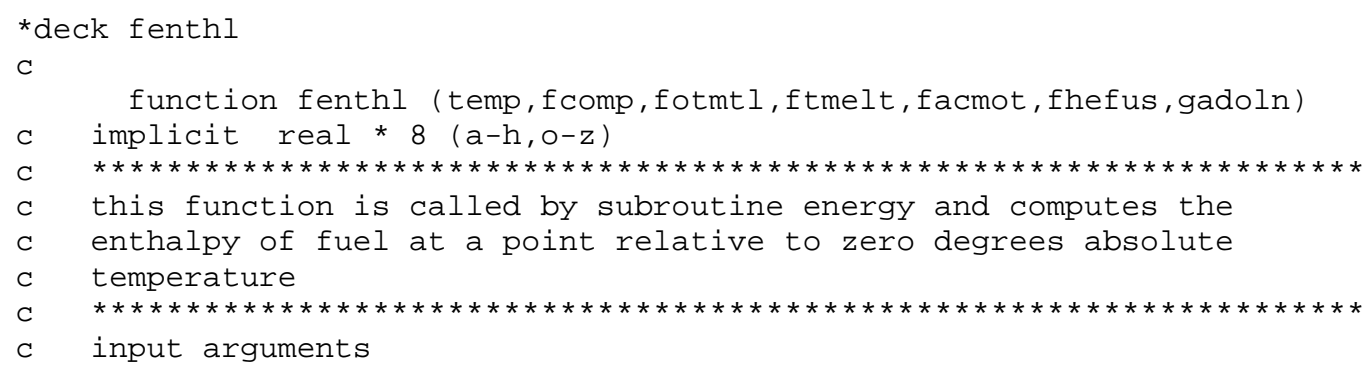




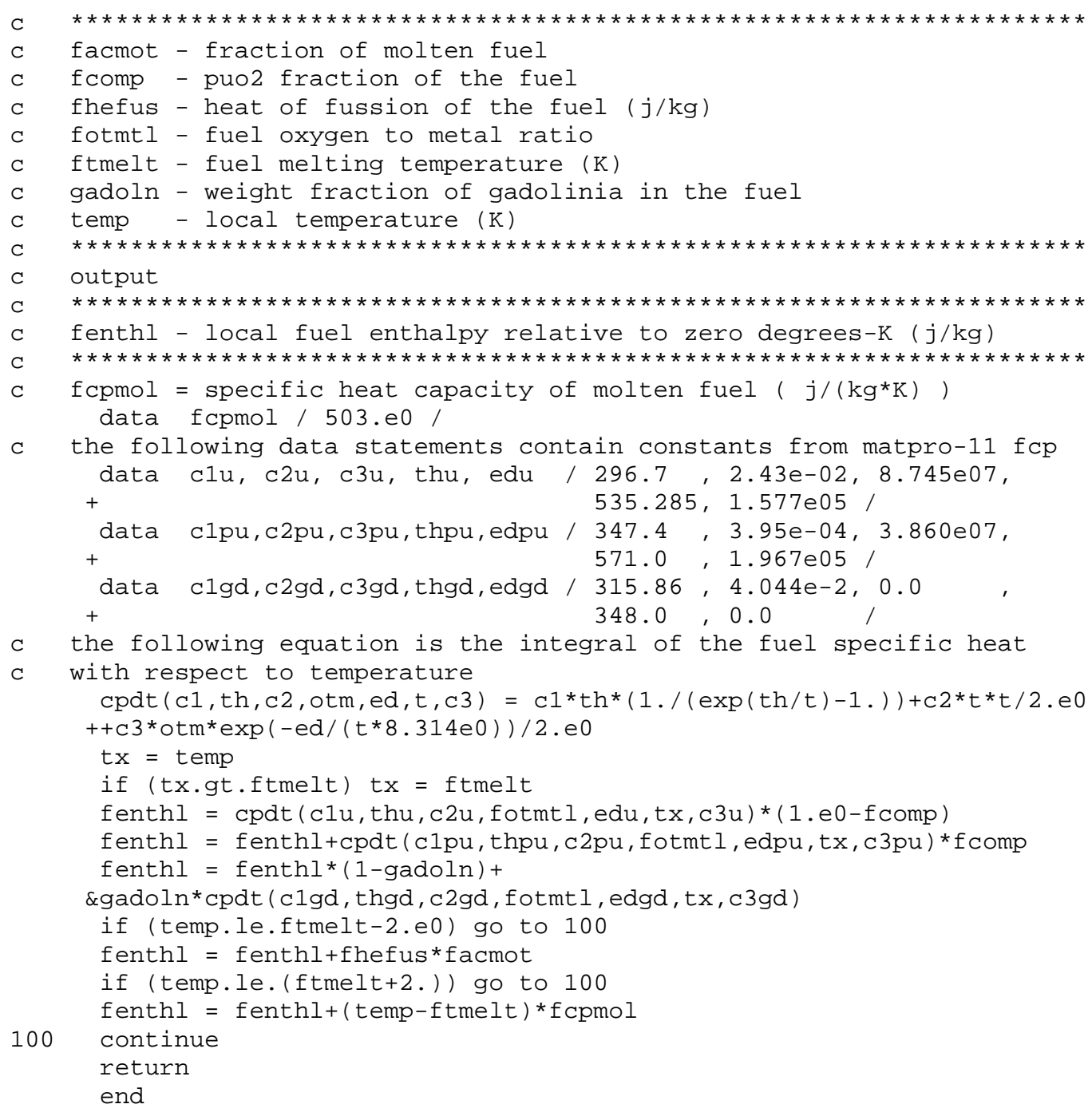

\section{FRAPTRAN 1.4: FENTHL}

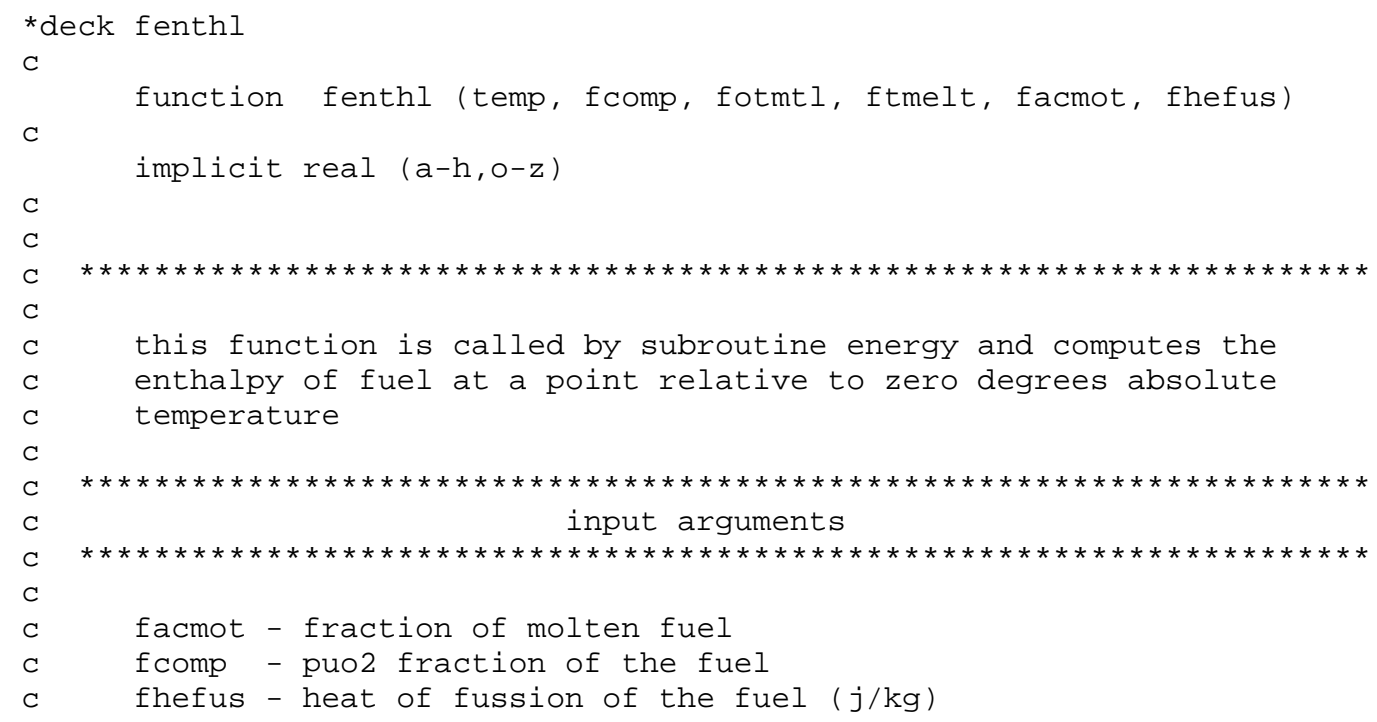




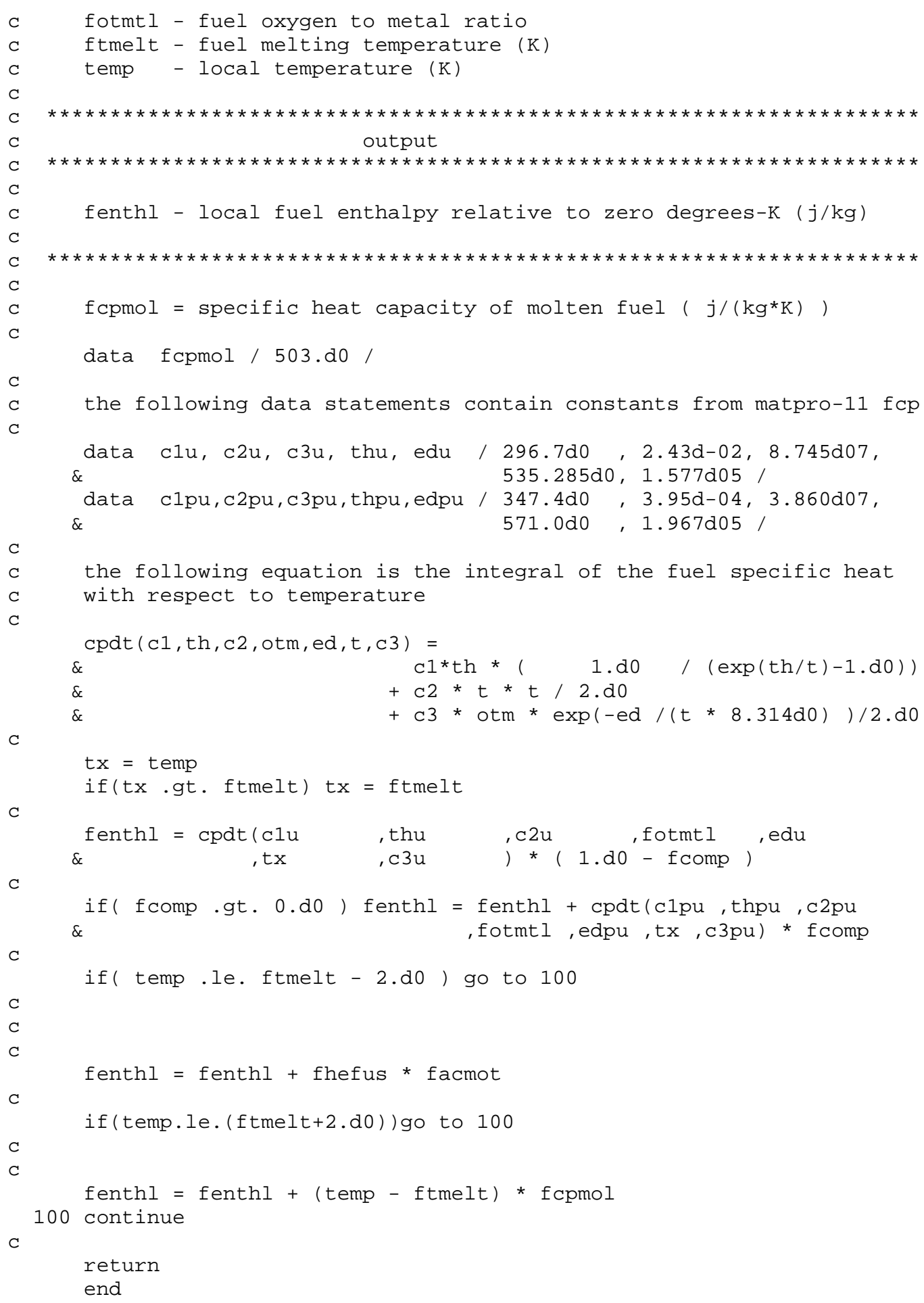

\section{A.1.3 Fuel Thermal Conductivity (FTHCON)}

\section{FRAPCON-3.4: FTHCON}

*deck fthcon

subroutine fthcon (ftemp, fraden, fotmtl, con, burnup

+ , gadoln, imox) 


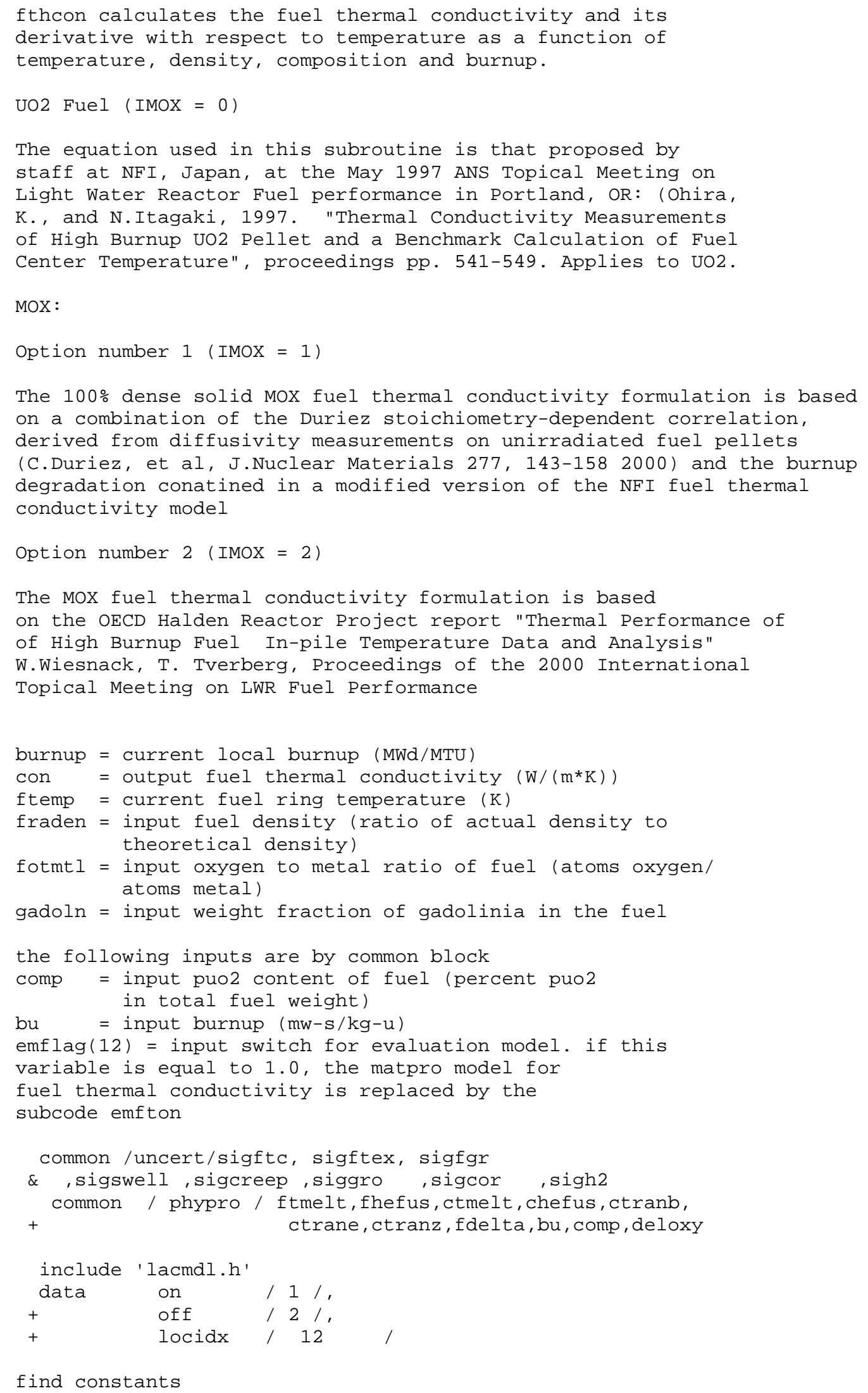


C

c Porosity correction is Lucuta correction, not Maxwell-Euken

$c$ as proposed by Duriez et al.

$x=2.0-$ fotmt 1

$\mathrm{ax}=2.85 * x+0.035$

C

$c x=\left(2.86-7.15^{*} x\right) * 1.0 \mathrm{e}-4$

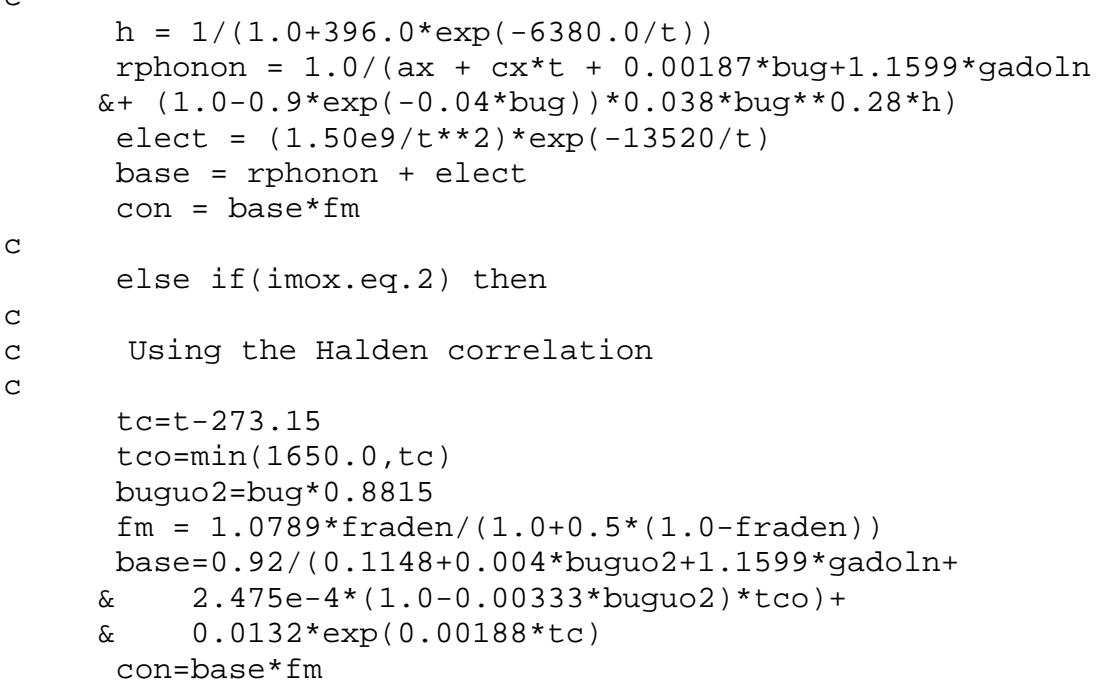




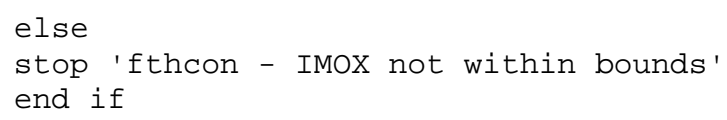

\section{FRAPTRAN 1.4: FTHCON}

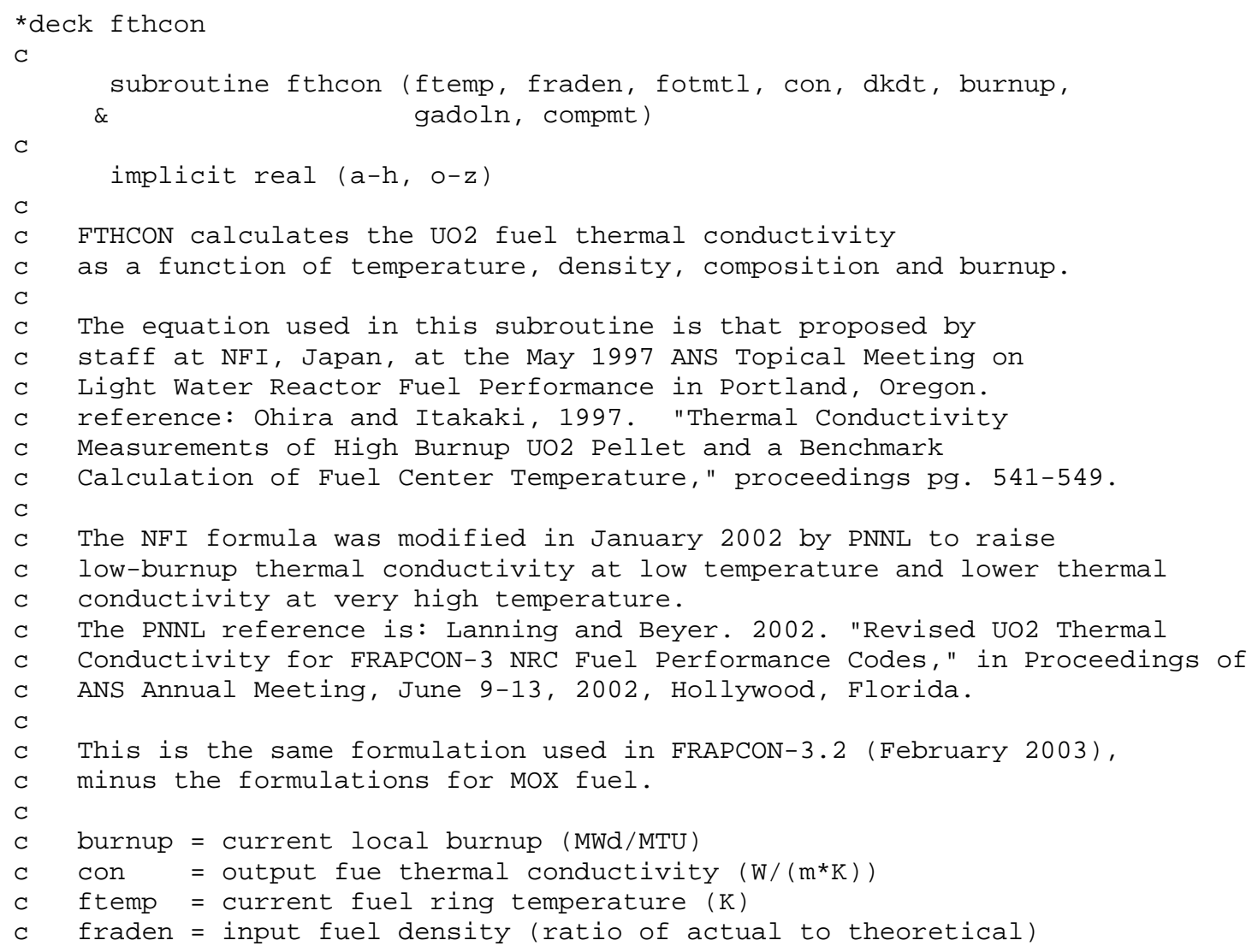




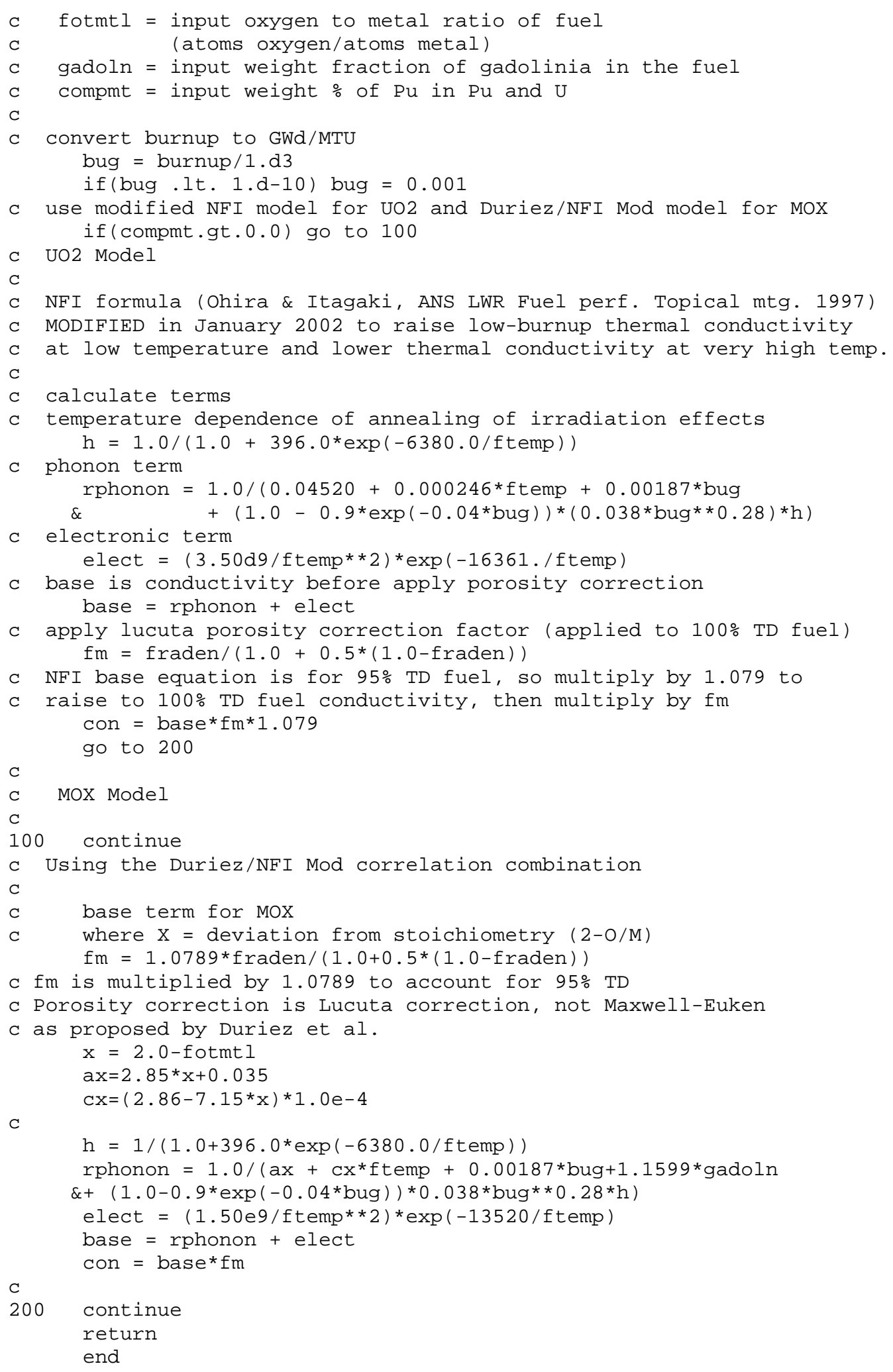

\section{A.1.4 Fuel Emissivity (FEMISS)}

\section{FRAPCON-3.4: FEMISS}




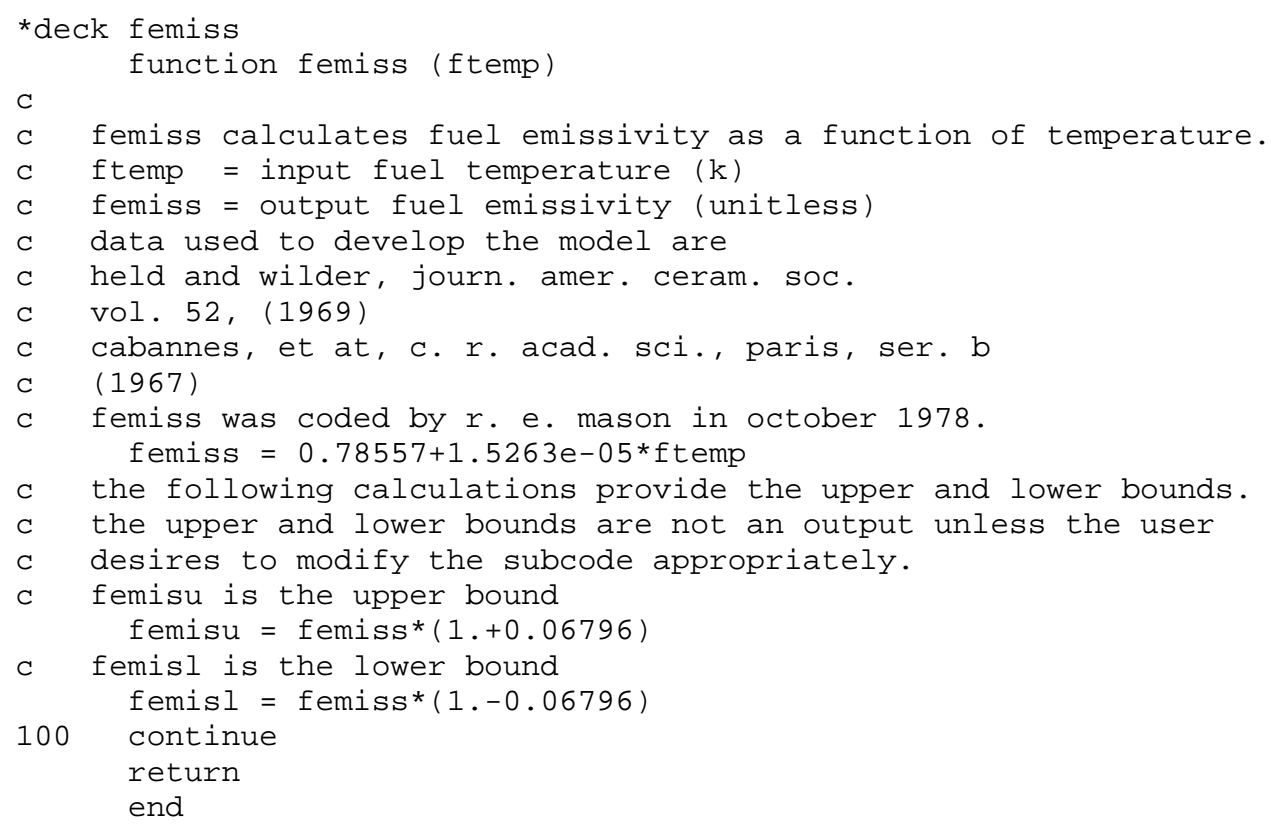

\section{FRAPTRAN 1.4: FEMISS}

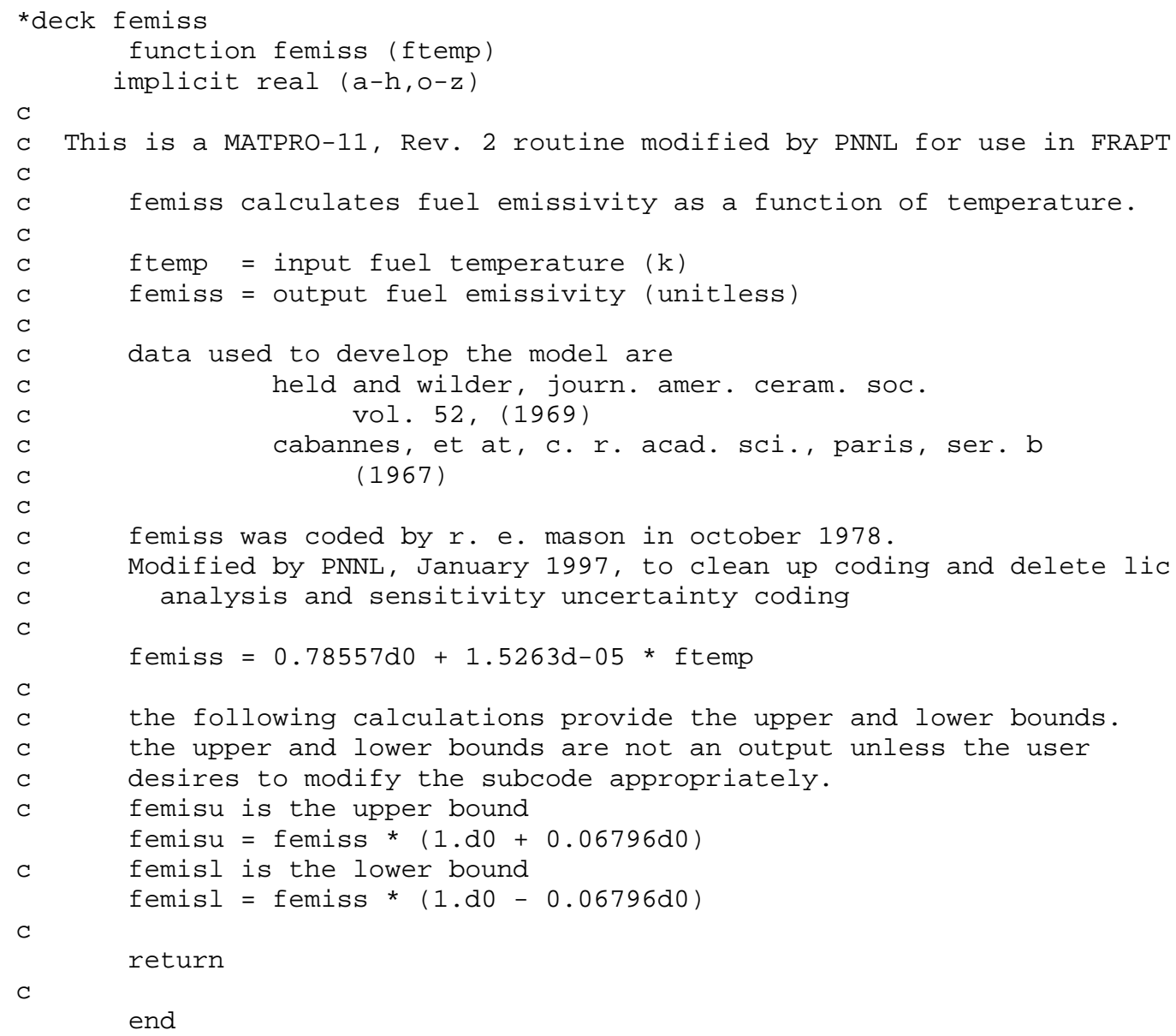




\section{A.1.5 Fuel Thermal Expansion (FTHEXP)}

\section{FRAPCON-3.4: FTHEXP}

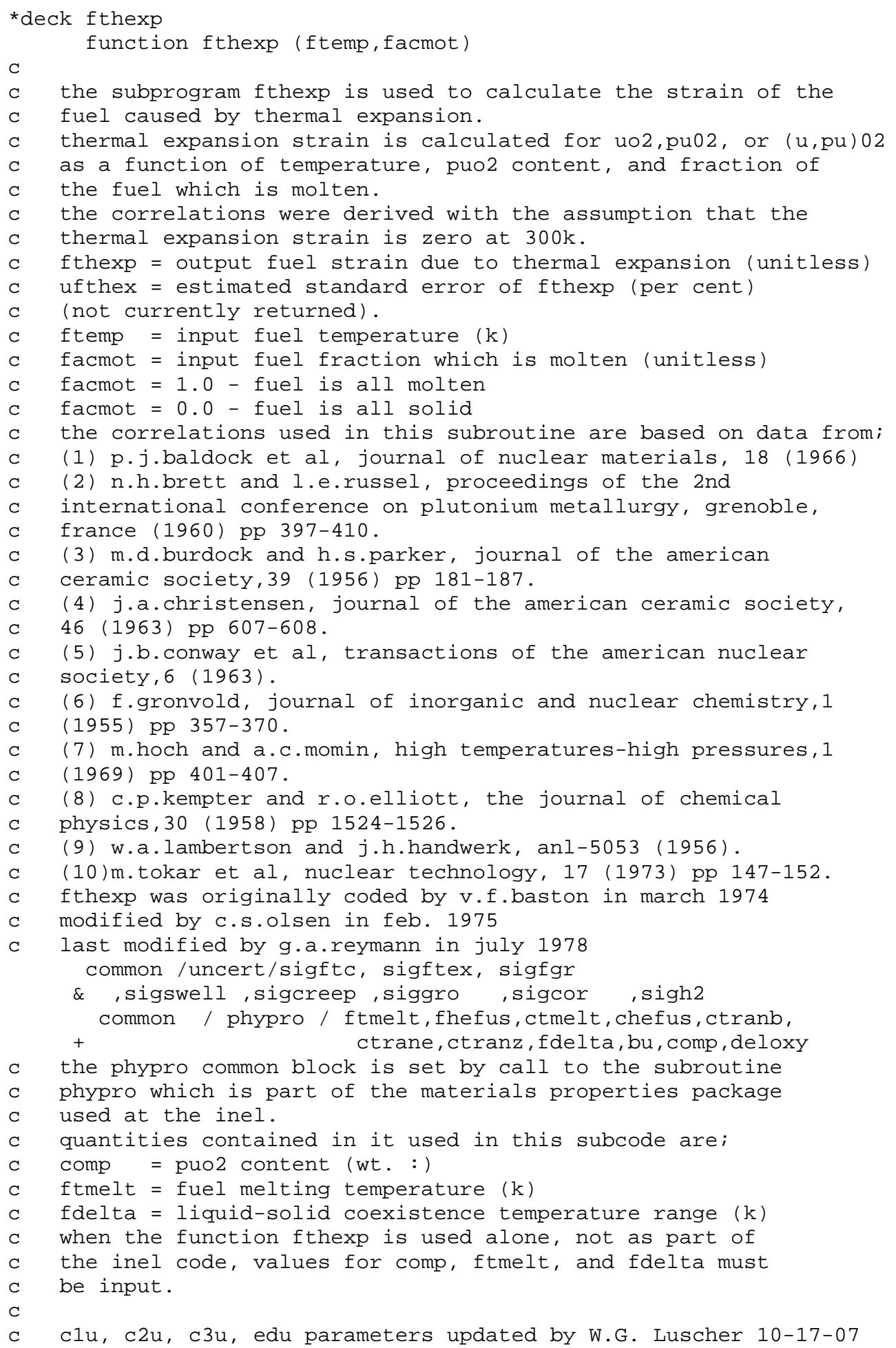

data c1u , c2u , c3u, edu / 9.8e-06, 2.61e-03, 3.16e-01, 1.32e-19 /

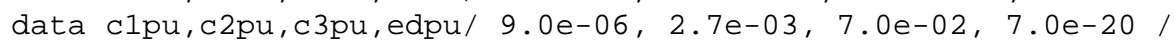




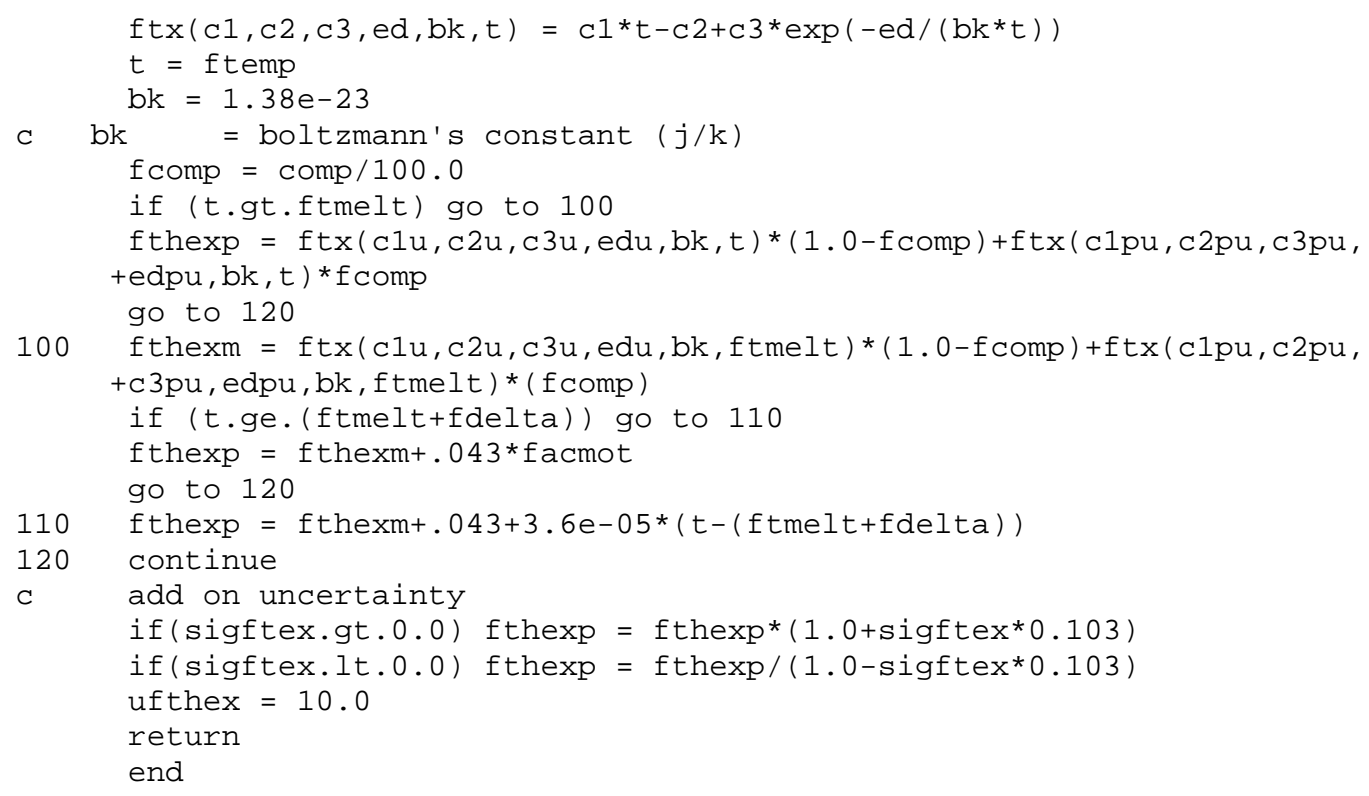

\section{FRAPTRAN 1.4: FTHEXP}

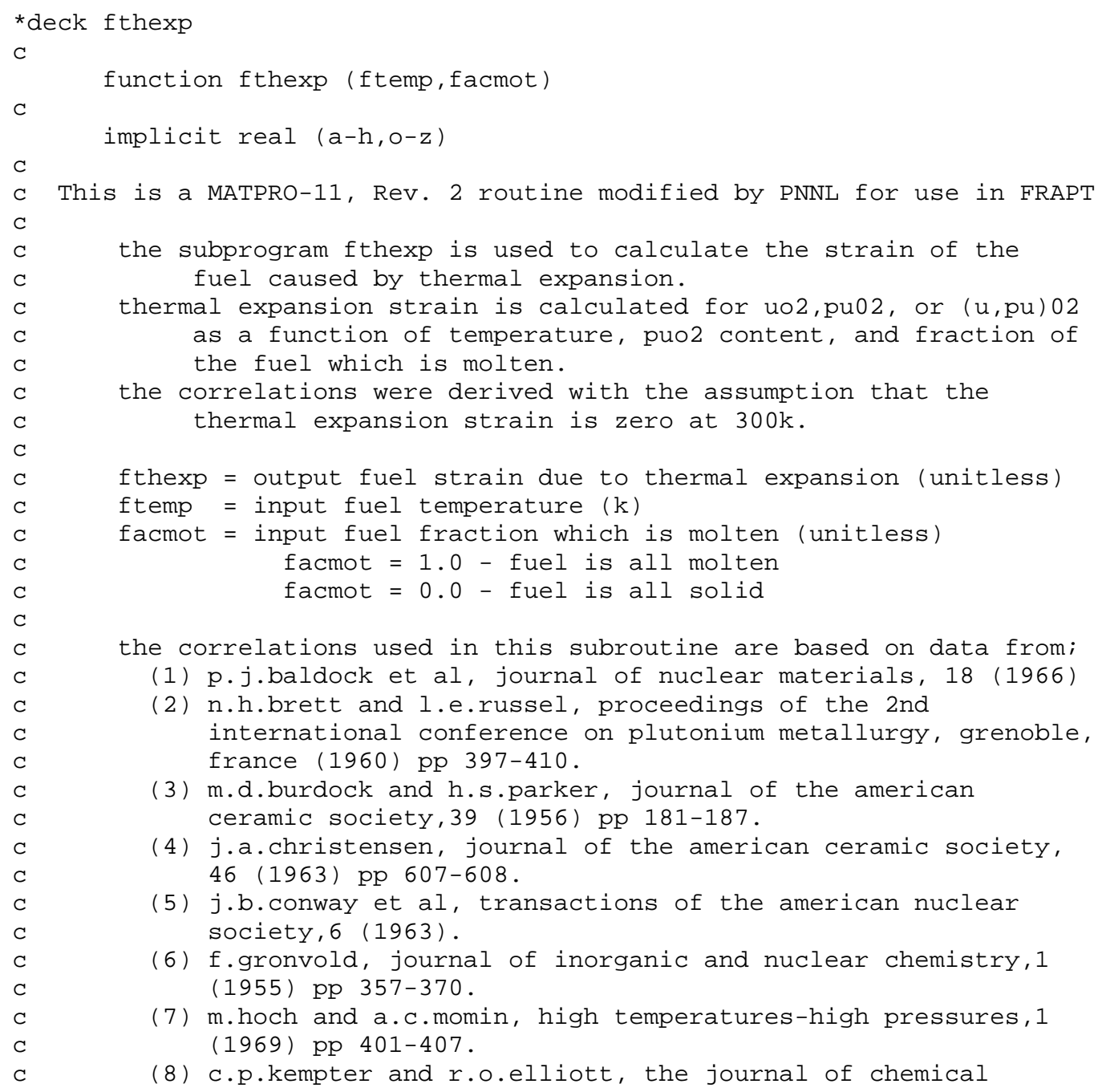




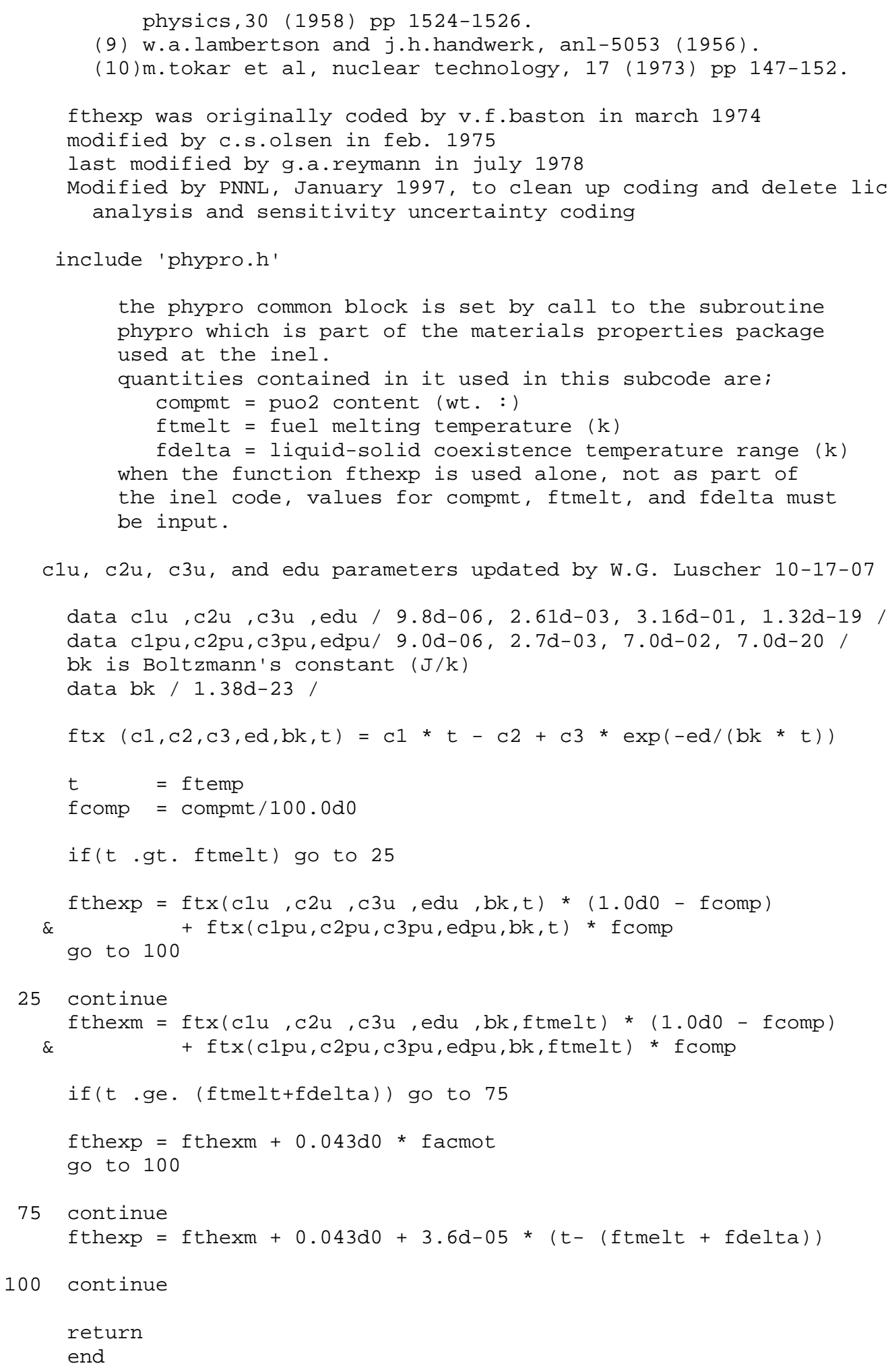

\section{A.1.6 Fuel Densification (FUDENS)}

FRAPCON-3.4: FUDENS 


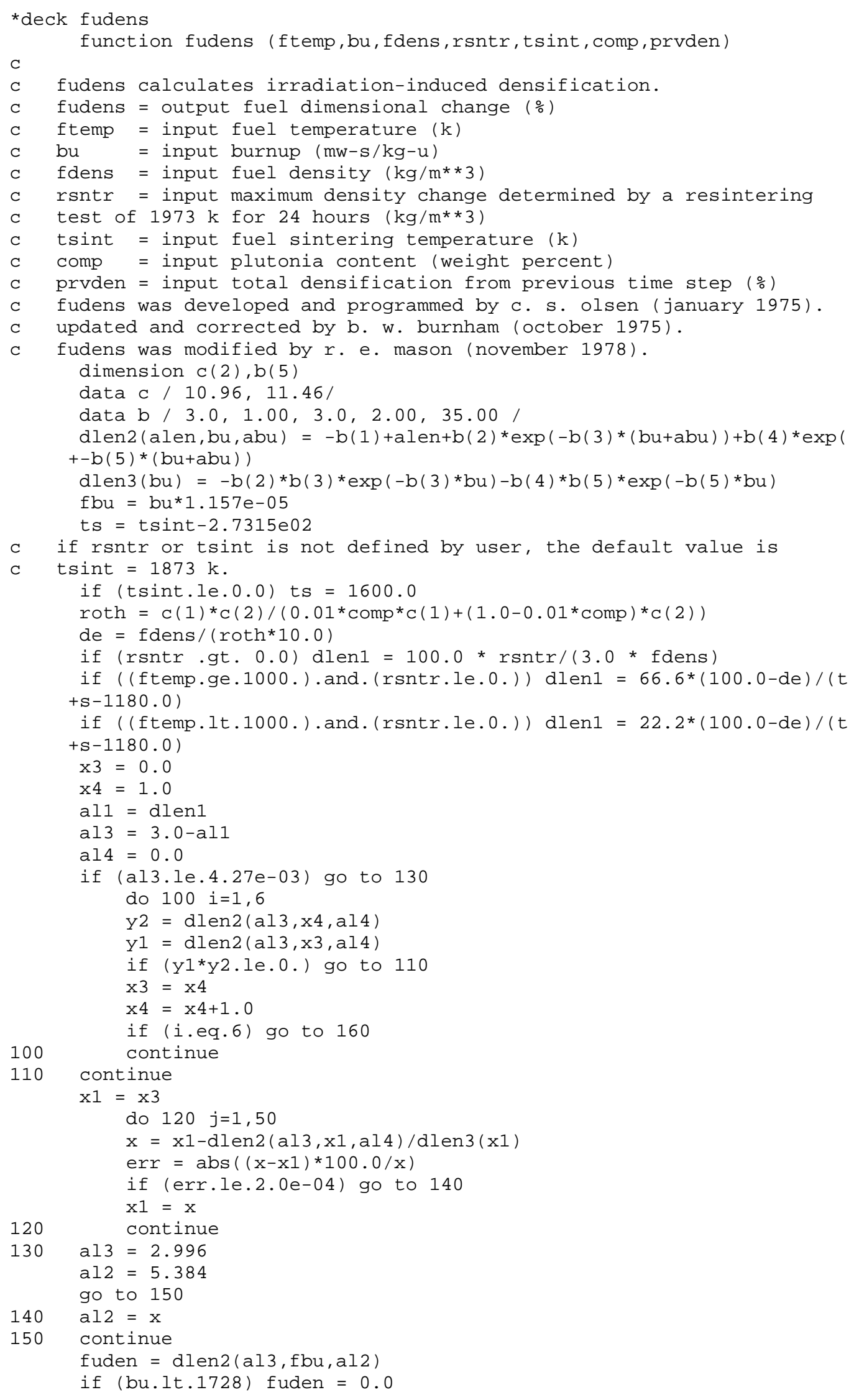




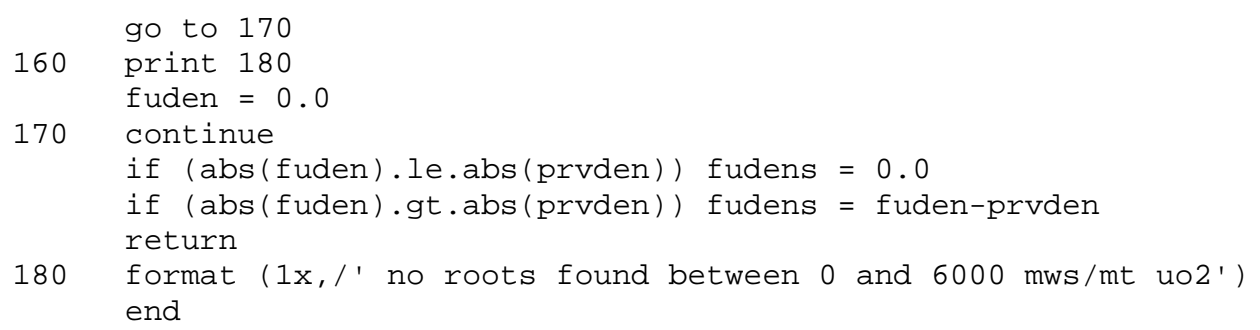

\section{A.1.7 Fuel Swelling (FSWELL)}

\section{FRAPCON-3.4: FSWELL}

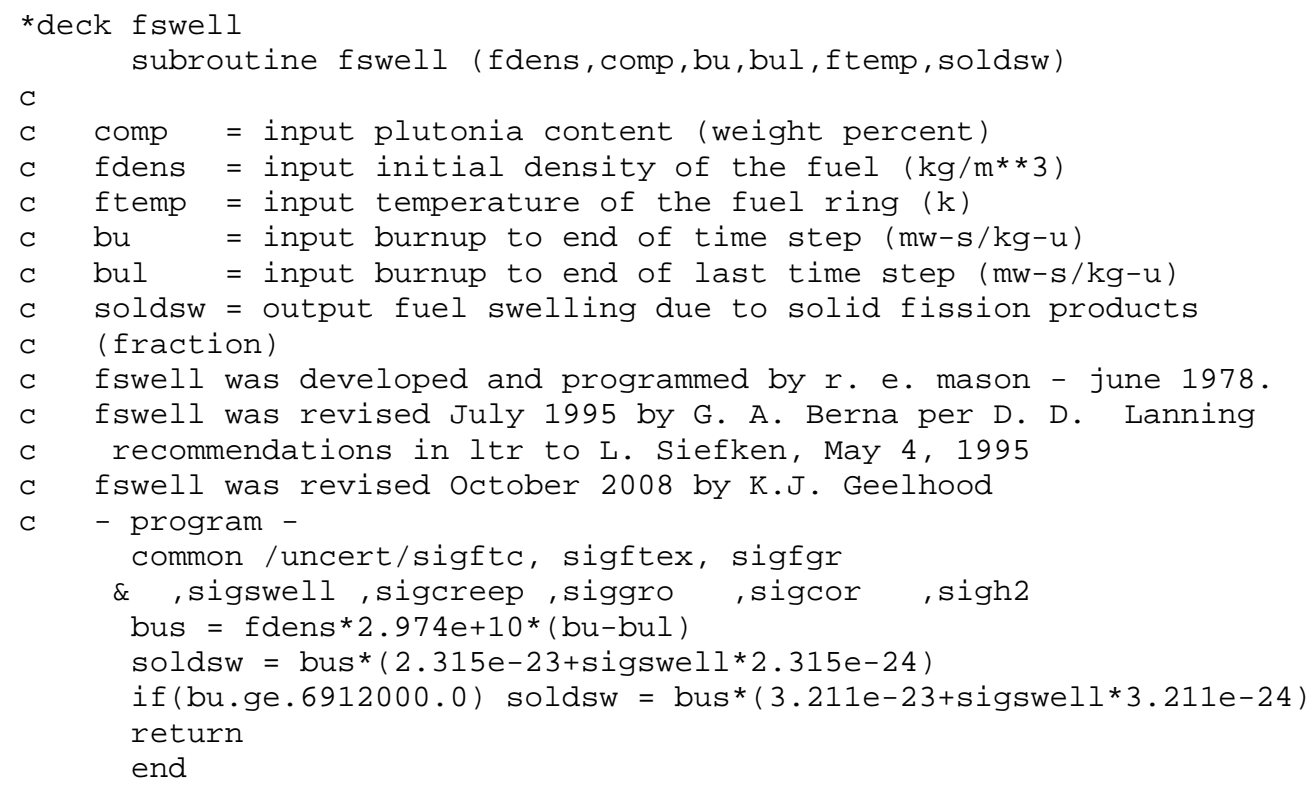

\section{A.2 Cladding Material Properties}

\section{A.2.1 Cladding Specific Heat (CCP)}

\section{FRAPCON-3.4: CCP}

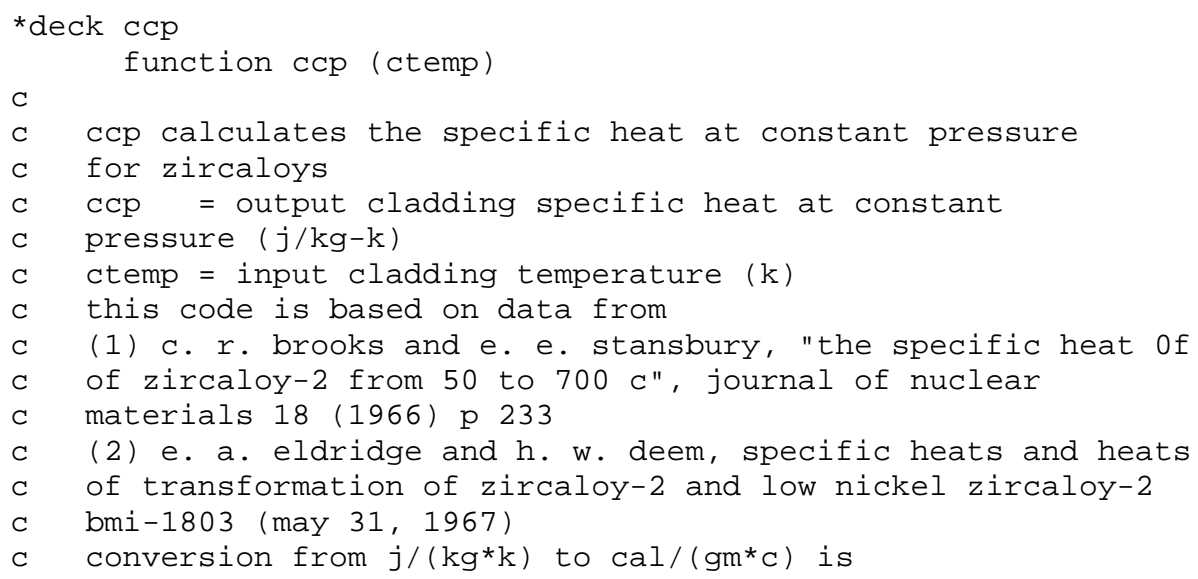




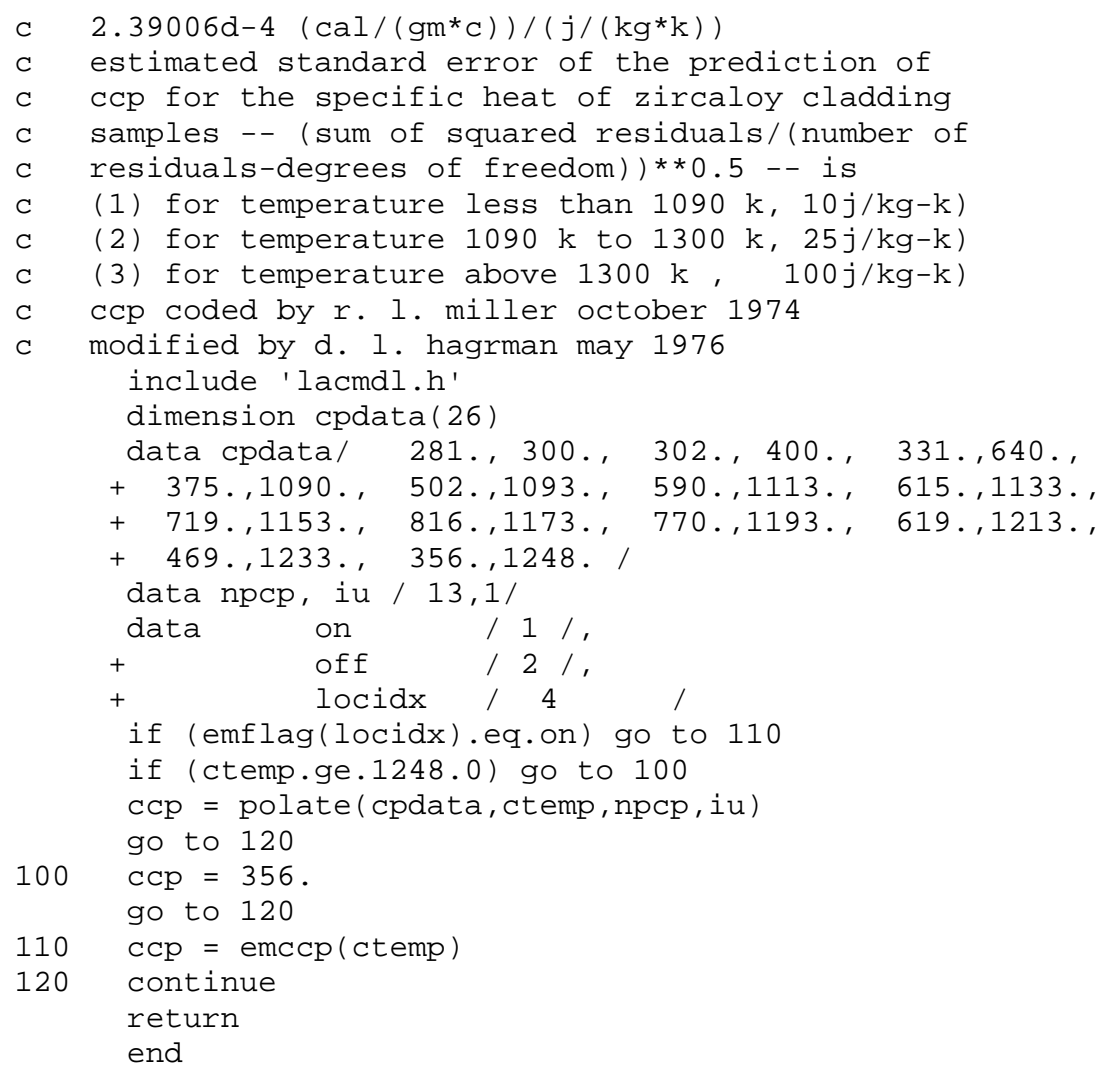

\section{FRAPTRAN 1.4: CCP}

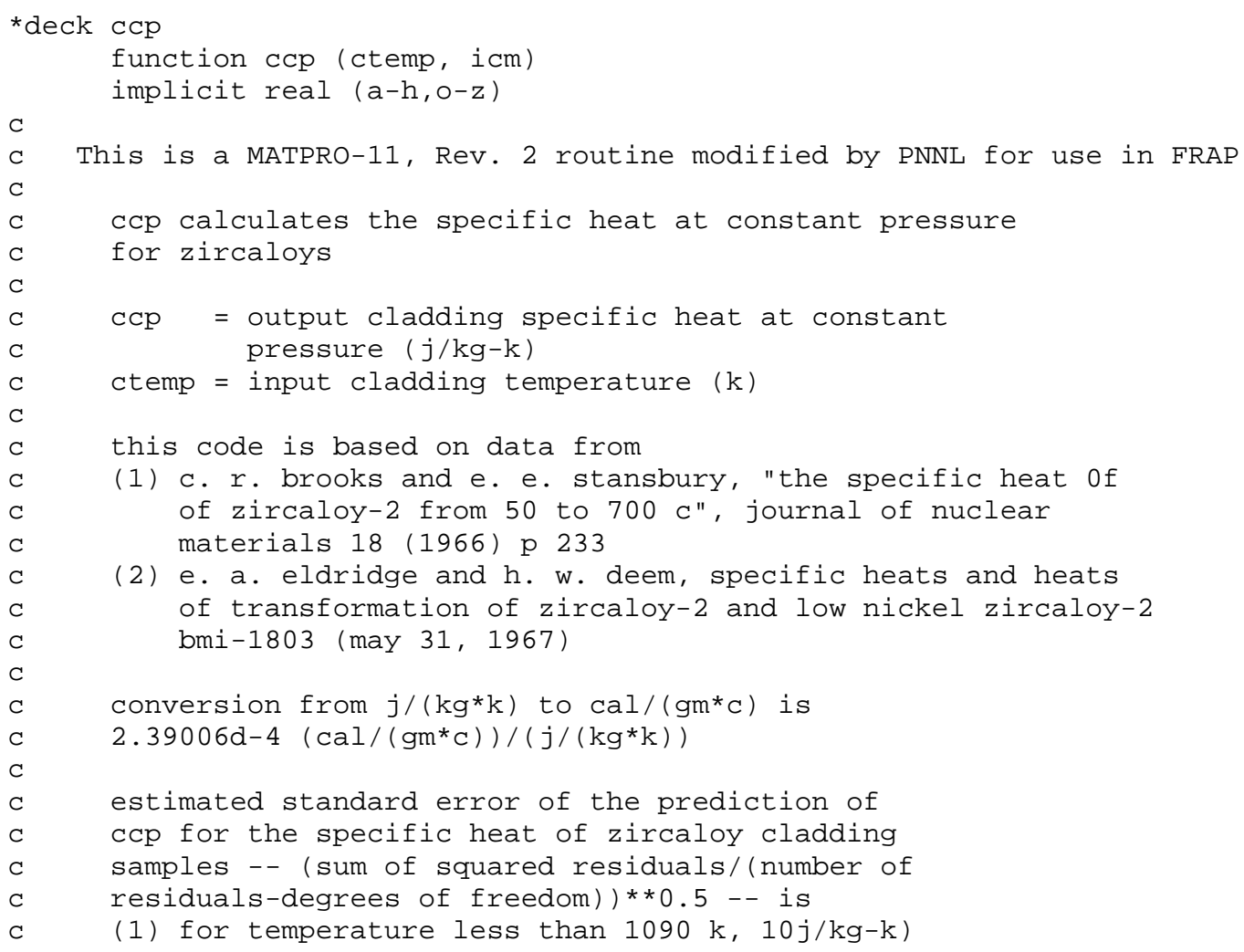




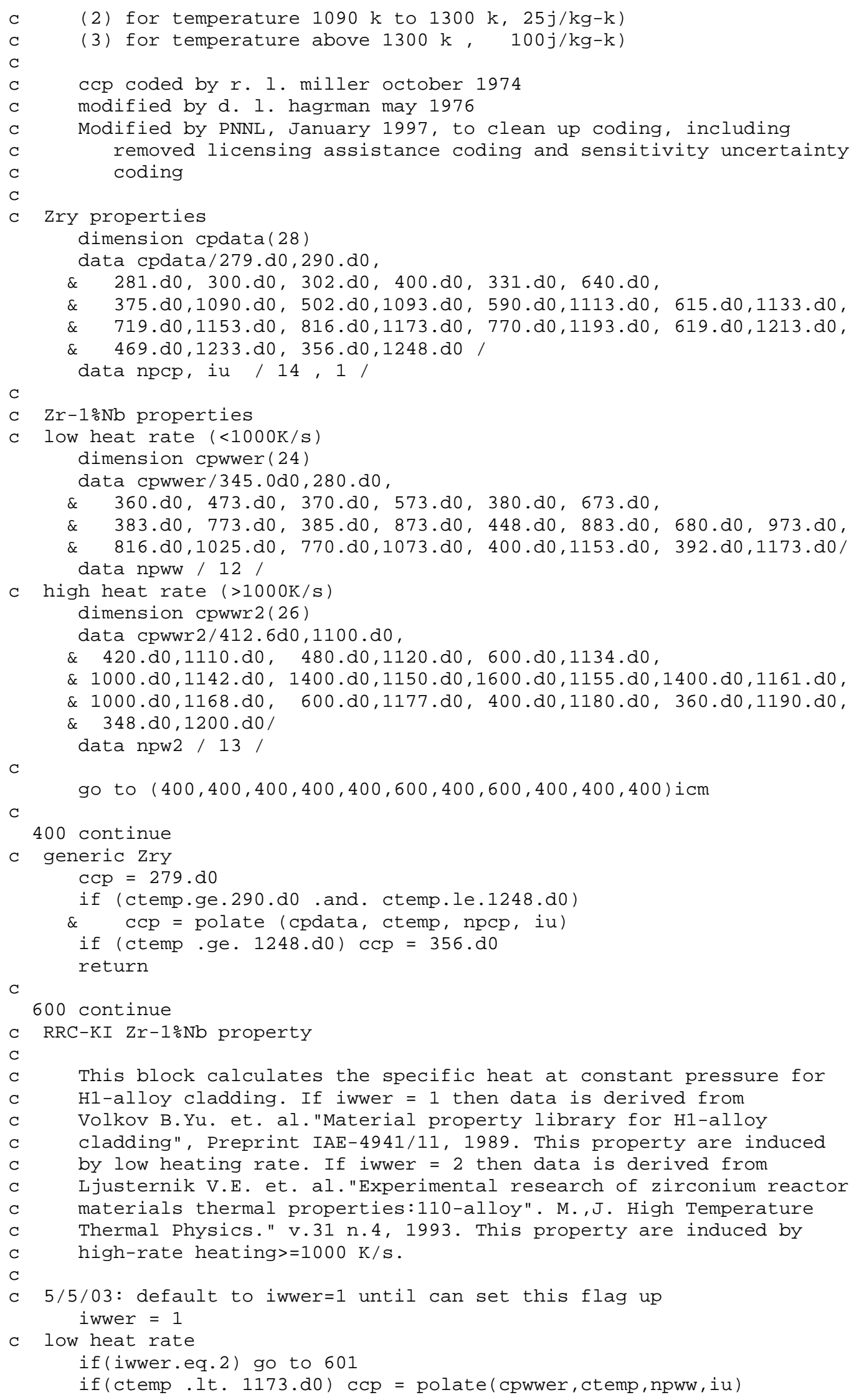




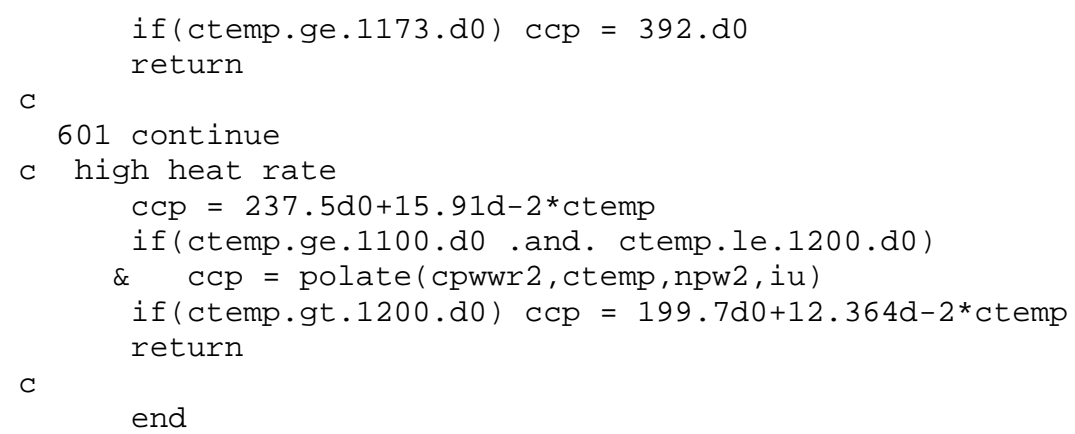

\section{A.2.2 Cladding Thermal Conductivity (CTHCON)}

\section{FRAPCON-3.4: (CTHCON)}

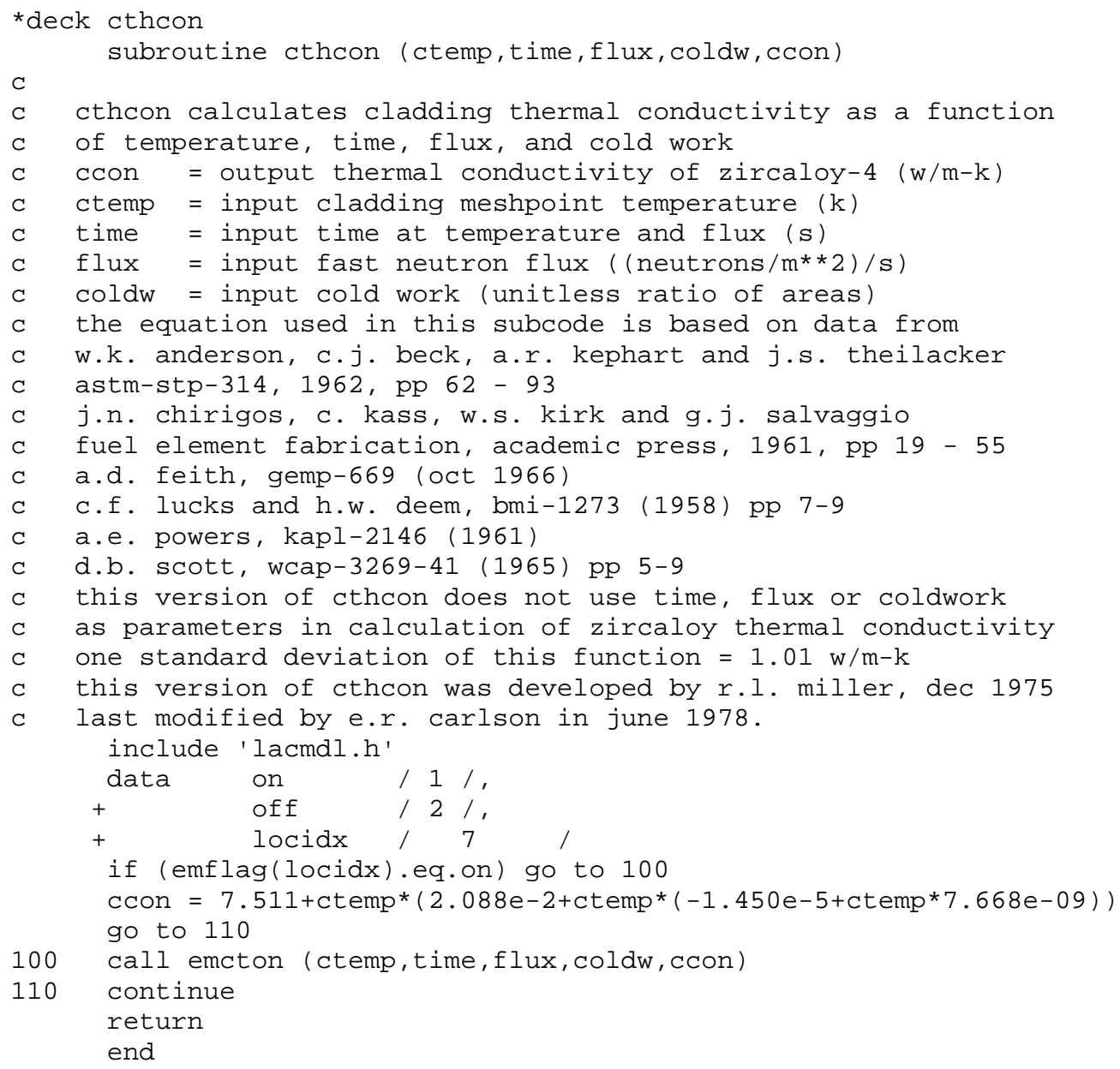

\section{FRAPTRAN 1.4: (CTHCON)}

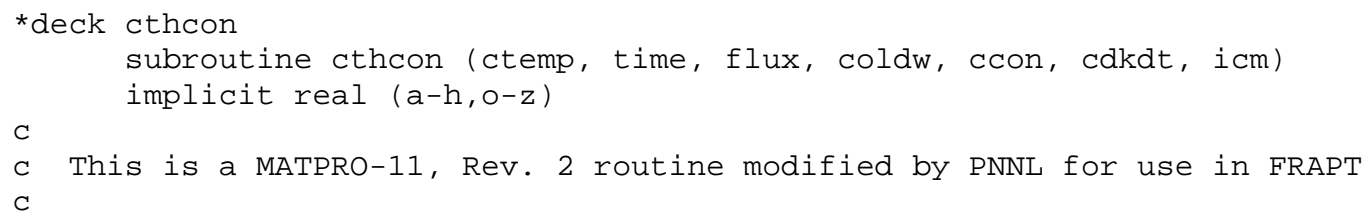




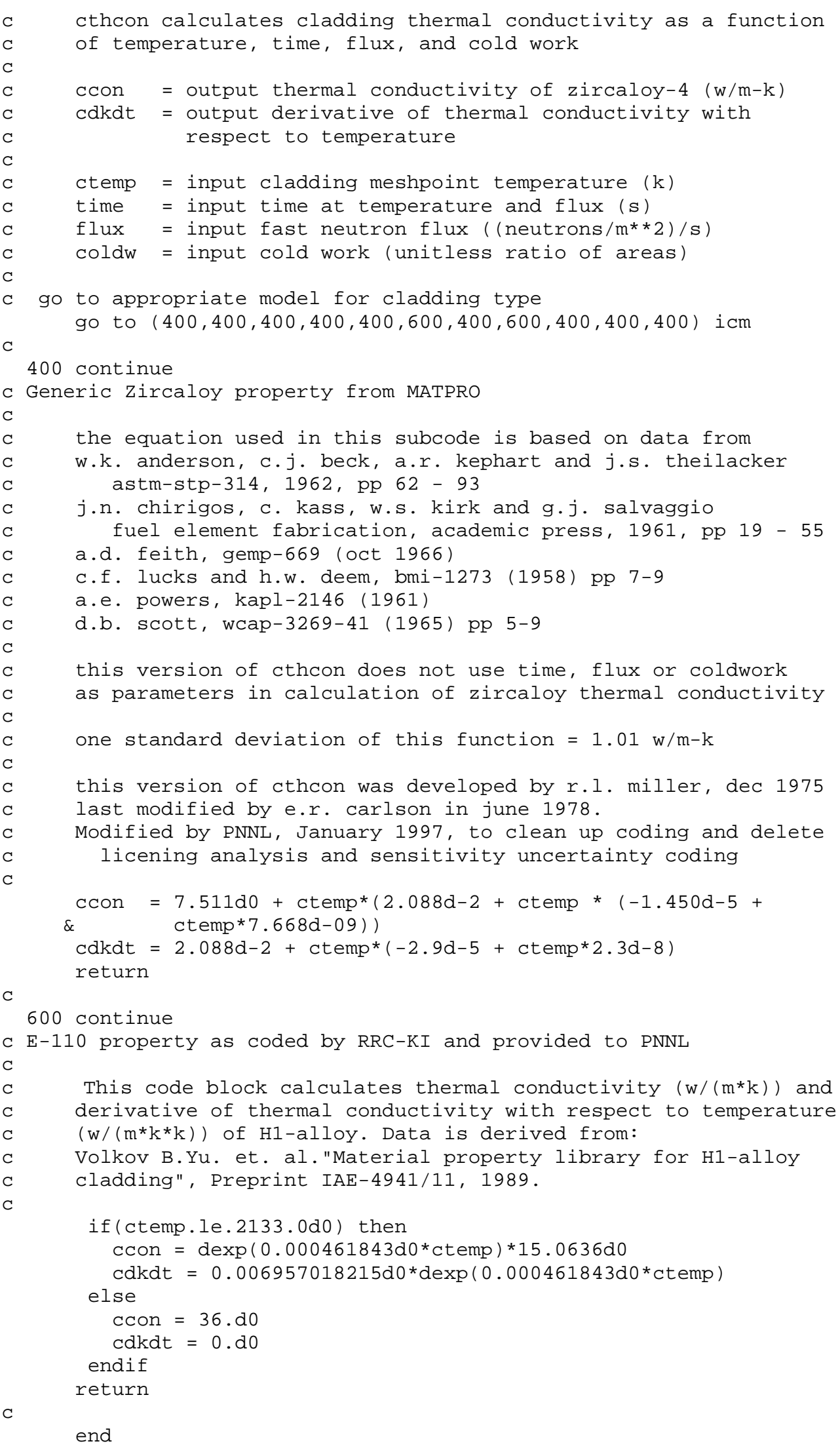




\section{A.2.3 Cladding Oxide Thermal Conductivity (ZOTCON)}

\section{FRAPCON-3.4: ZOTCON}

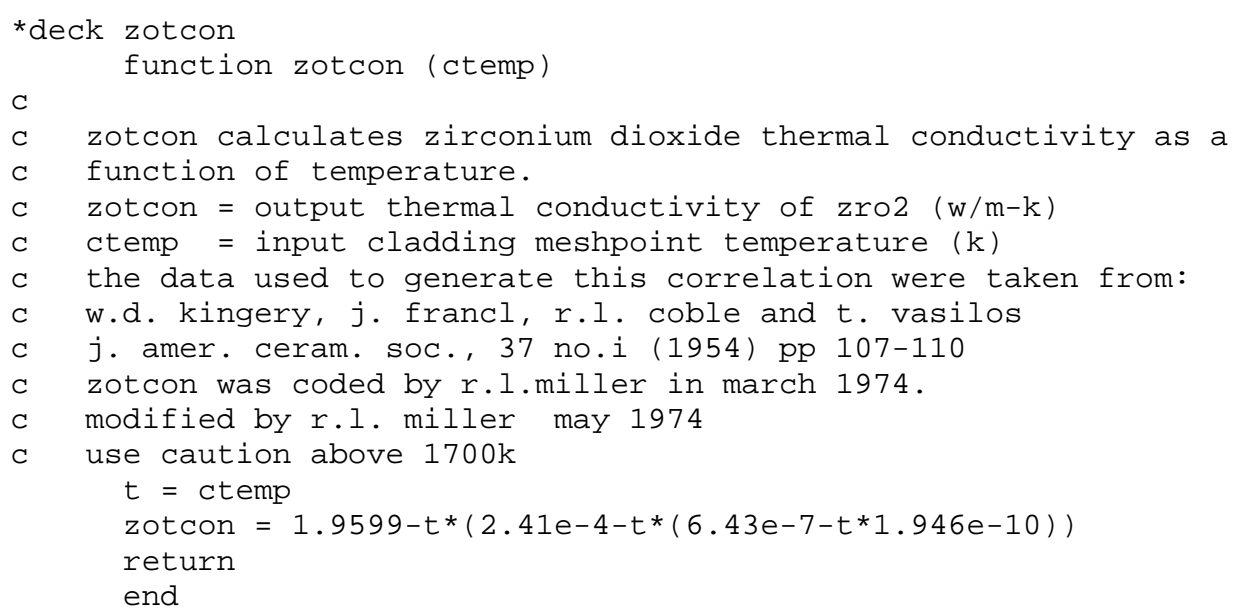

\section{FRAPTRAN 1.4: ZOTCON}

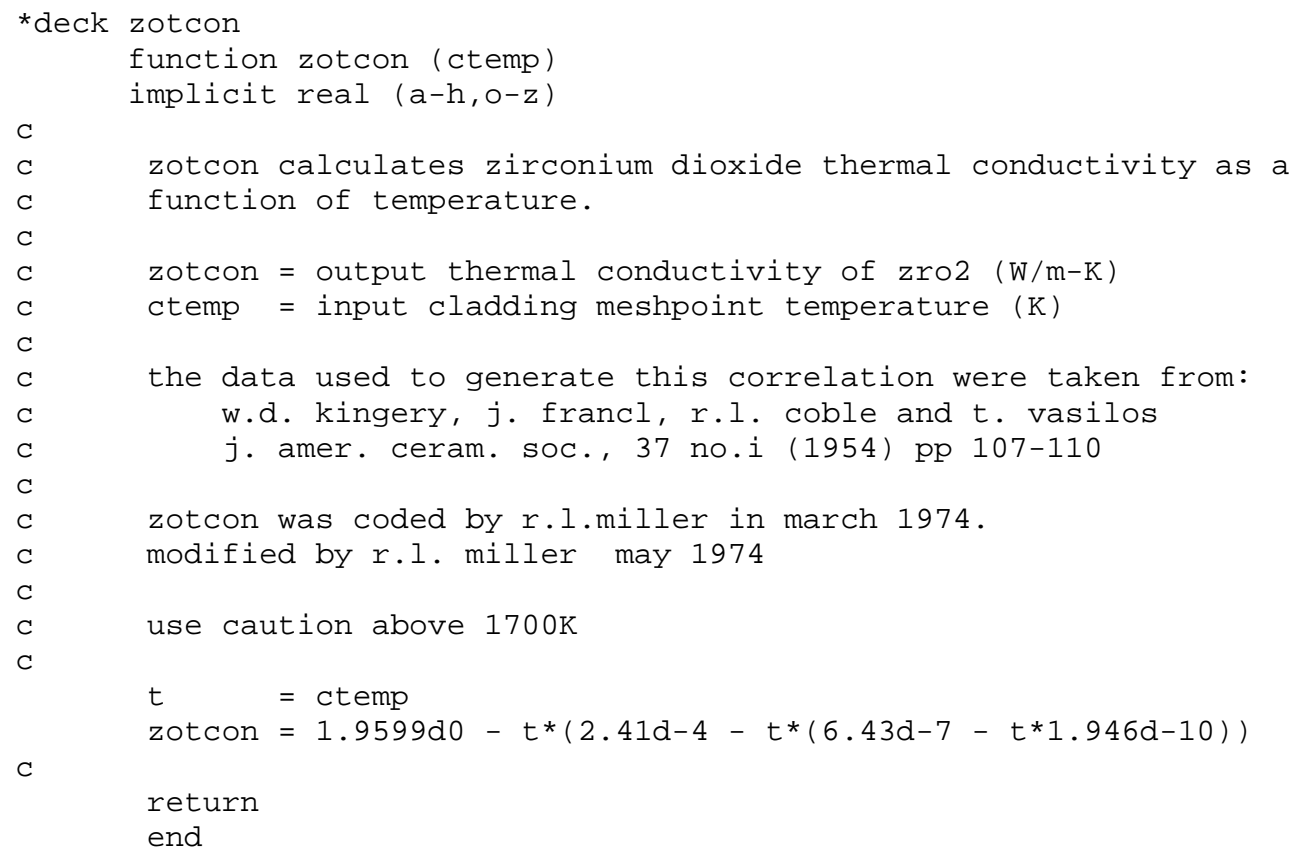

\section{A.2.4 Cladding Oxide Emissivity (ZOEMIS)}

\section{FRAPCON-3.4: ZOEMIS}

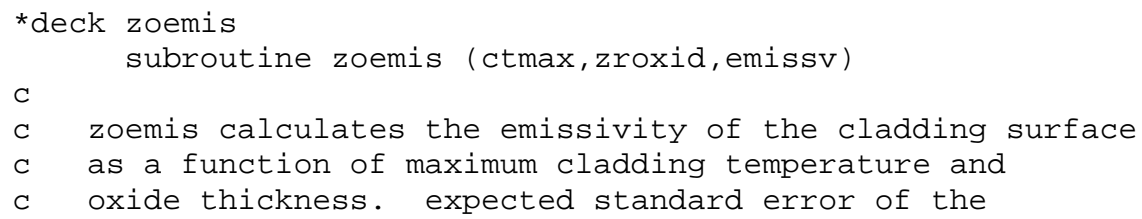




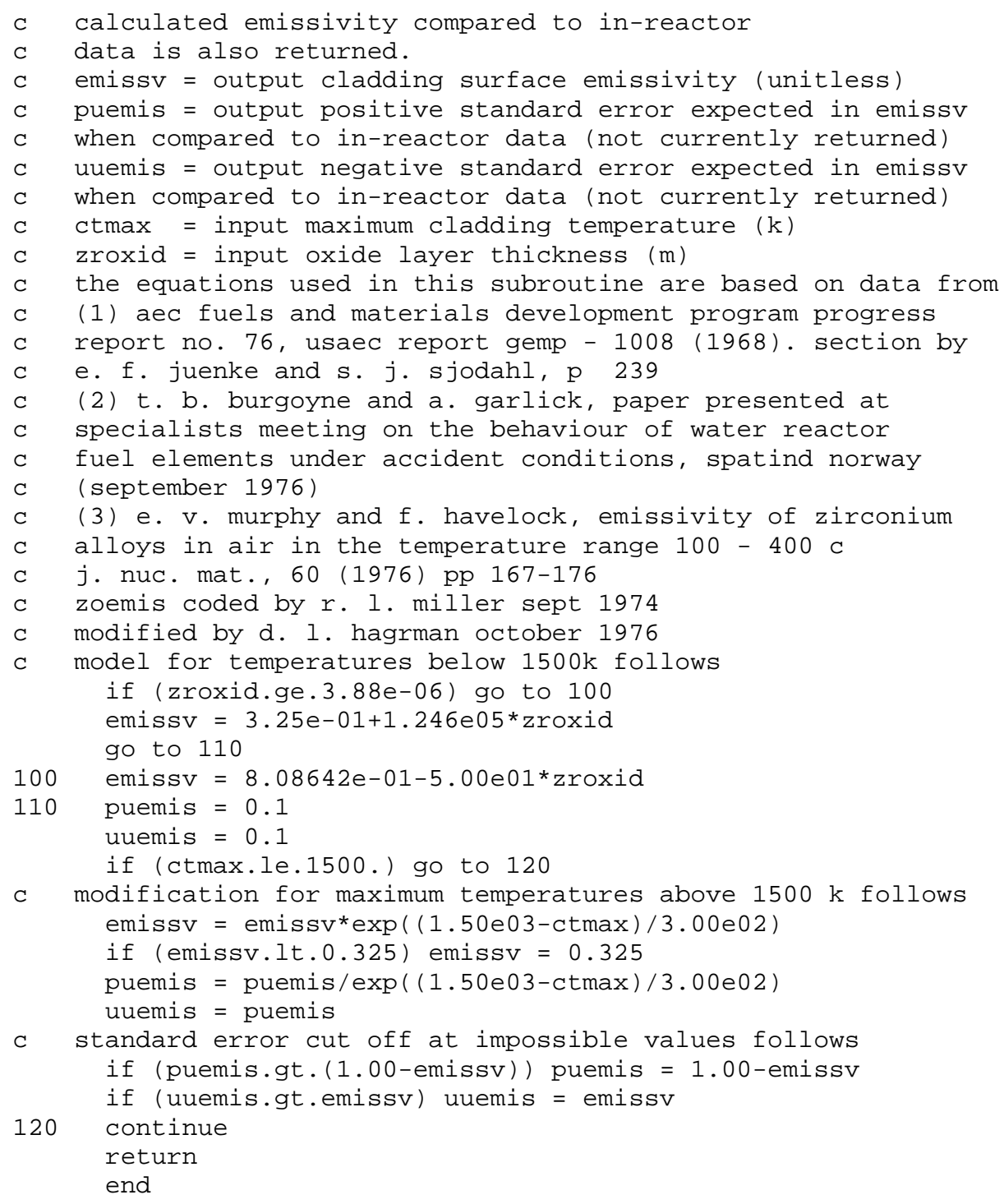

\section{FRAPTRAN 1.4: ZOEMIS}

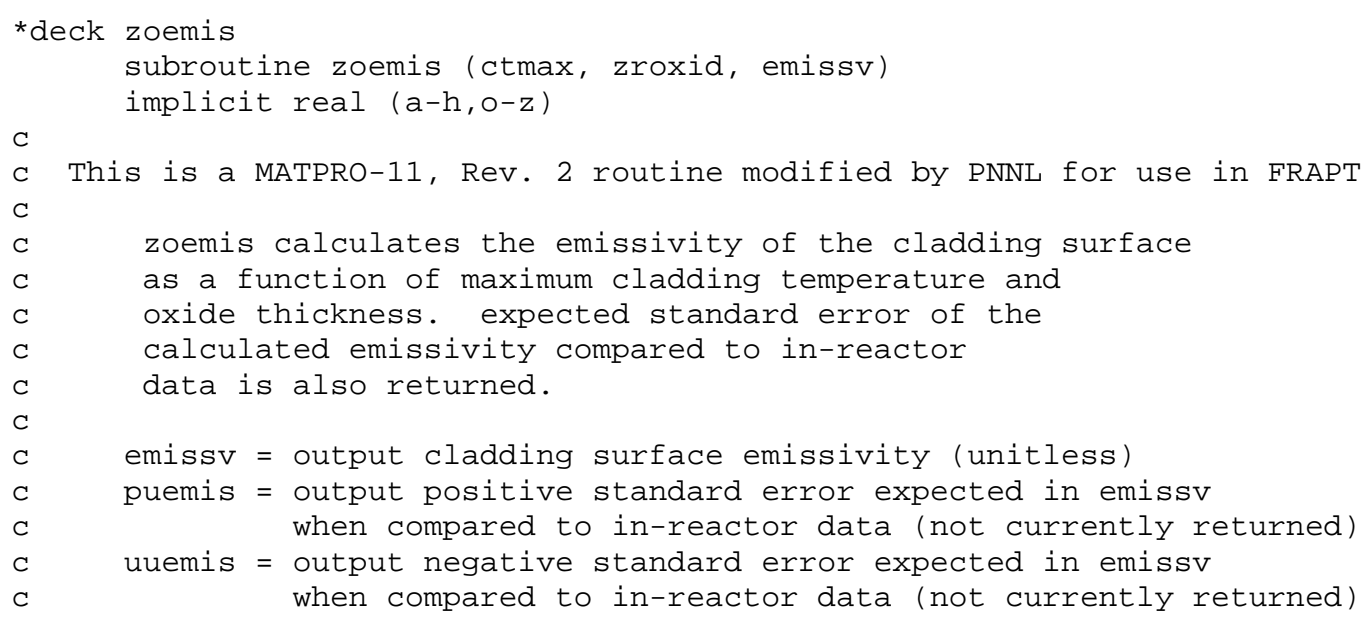




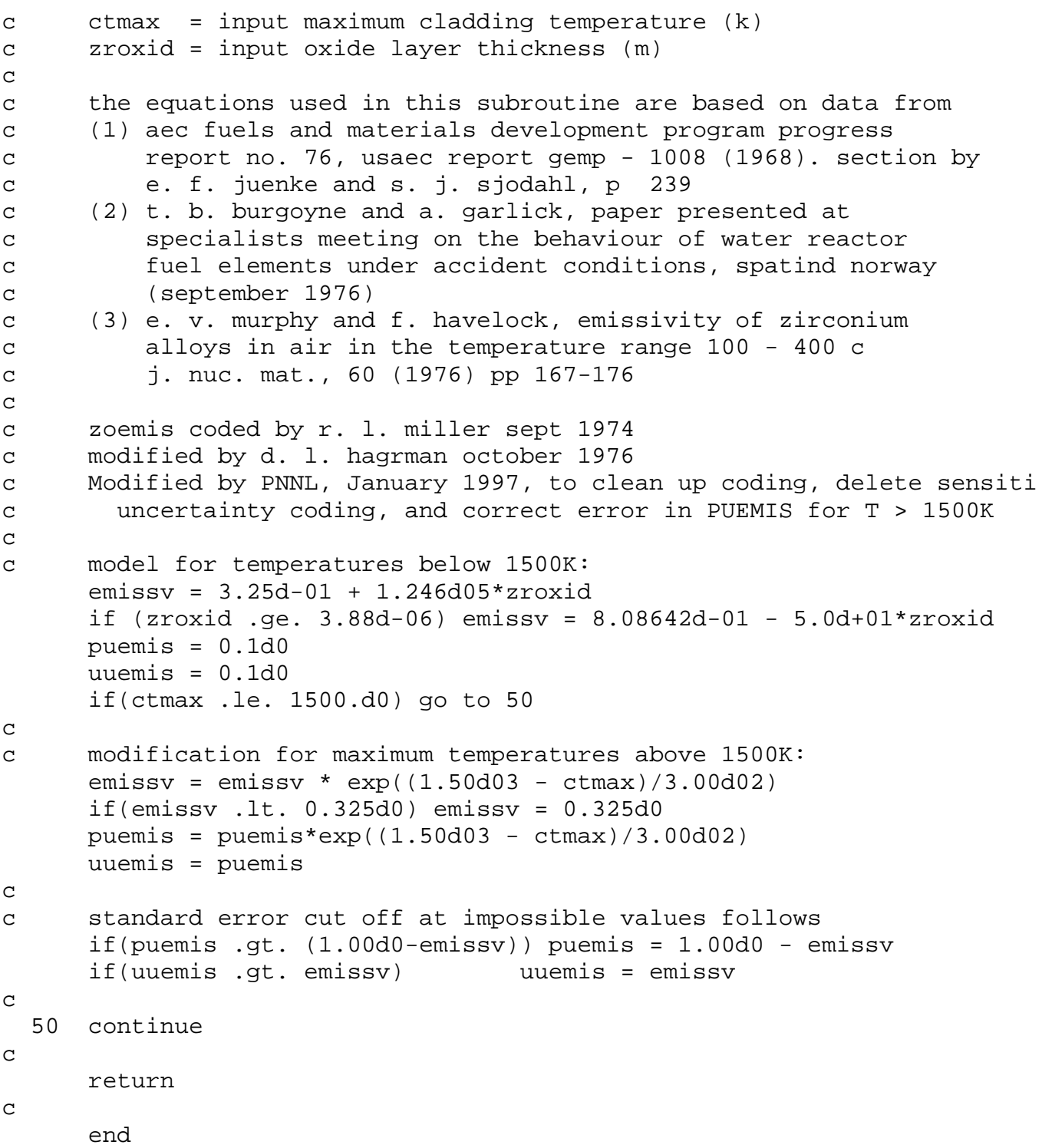

\section{A.2.5 Cladding Thermal Expansion (CTHEXP)}

\section{FRAPCON-3.4: CTHEXP}

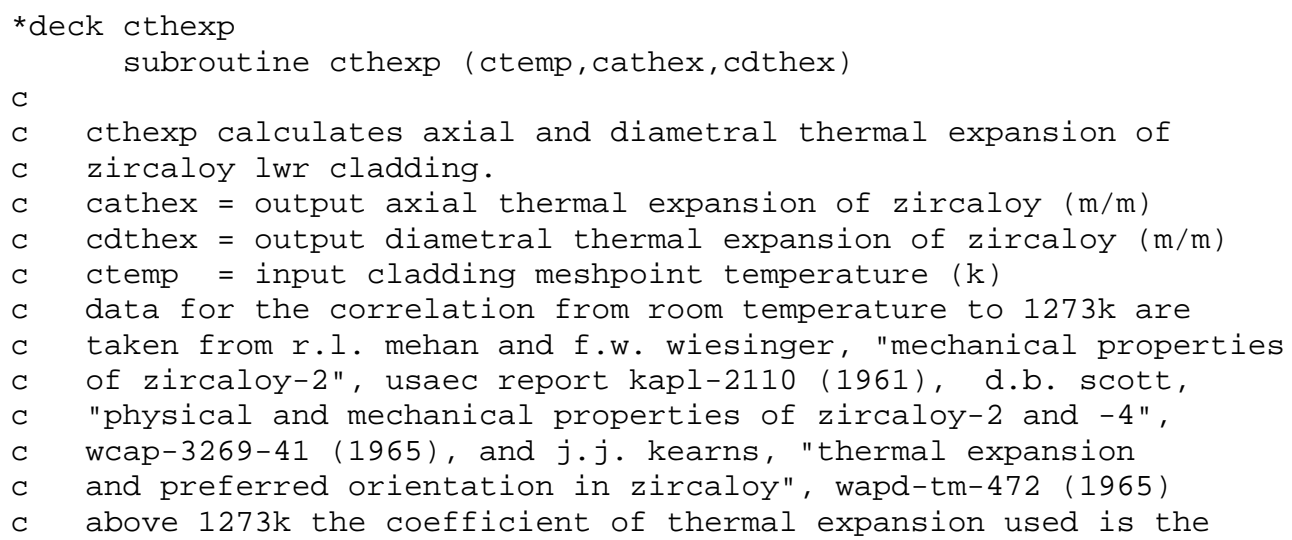


c constant value $9.7 e-06 / \mathrm{k}$ recommended by b. lustman and $f$. kerze, c "the metallurgy of zirconium", mc graw-hill book company, new c york (1955) page 355.

c between 1073 and 1273k (approximately the alpha-beta transition c range for zircaloy) cthexp uses the linear interpolation routine c polate to find the thermal expansion.

c cthexp was originally coded by r.l. miller in nov. 1974.

last updated by g.a. reymann in june 1978.

dimension cathxd (44)

dimension dthexp (44)

data cathxd/

$+\quad 3.52774 \mathrm{e}-03$

$+\quad 3.50000 \mathrm{e}-03$,

$+\quad 3.41000 \mathrm{e}-03$,

$+\quad 3.21000 \mathrm{e}-03$,

$+\quad 2.80000 \mathrm{e}-03$,

$+\quad 2.00000 \mathrm{e}-03$,

$+\quad 1.30000 \mathrm{e}-03$,

$+\quad 1.13000 \mathrm{e}-03$,

$+\quad 1.11000 \mathrm{e}-03$

$+\quad 1.20000 \mathrm{e}-03$,

$+\quad 1.40000 \mathrm{e}-03$,

data npcatx, iu / 22, 1

data dthexp/

+ 5.1395e-03, 1073.15,

1073.15
1093.15
1113.15
1133.15
1153.15
1173.15
1193.15
1213.15
1233.15
1253.15
1273.15

๑. $0, \quad 293.15$

$3.53000 \mathrm{e}-03, \quad 1083.15$

3. $46000 \mathrm{e}-03$,

1103.15

3.33000e-03, $\quad 1123.15$

3. $07000 \mathrm{e}-03, \quad 1143.15$

2.50000e-03, $\quad 1163.15$

$1.50000 \mathrm{e}-03, \quad 1183.15$

1. $16000 \mathrm{e}-03$,

1203.15

1. $10000 \mathrm{e}-03$,

1223.15

1. $13000 \mathrm{e}-03$,

1243.15

1.30000e-03,

1263.15

$+5.2800 \mathrm{e}-03,1103.15$,

$+5.2200 \mathrm{e}-03,1133.15$,

5. 2200e-03, 1083.15,

4.3480e-04, 373.15,

5.2800e-03, 1113.15,

5.2500е-03, 1093.15,

$+4.9000 \mathrm{e}-03,1163.15$,

5.1500е-03, 1143.15,

5. $0800 \mathrm{e}-03,1153.15$,

$+4.1000 \mathrm{e}-03,1193.15$,

$4.7000 \mathrm{e}-03,1173.15$,

4.4500e-03, 1183.15,

$+2.9700 \mathrm{e}-03,1223.15$,

3. $5000 \mathrm{e}-03,1203.15$,

3.1300e-03, 1213.15,

2.9200e-03, 1233.15, 2.8700e-03, 1243.15,

$+2.8600 \mathrm{e}-03,1253.15$,

2.8800e-03, 1263.15,

2. $9000 \mathrm{e}-03,1273.15 /$ data npdexp / 22 /

if (ctemp.le.1073.15) go to 100

if (ctemp.ge.1273.15) go to 110

cathex $=$ polate $($ cathxd, ctemp, npcatx, iu $)$

cdthex $=$ polate $($ dthexp, ctemp, npdexp, iu $)$

go to 120

100 cathex $=-2.5060 \mathrm{e}-05+($ ctemp -273.15$) * 4.4410 \mathrm{e}-06$

cdthex $=-2.3730 \mathrm{e}-04+($ ctemp -273.15$) * 6.7210 \mathrm{e}-06$

go to 120

110 cathex $=-8.300 \mathrm{e}-03+($ ctemp -273.15$) * 9.70 \mathrm{e}-06$

cdthex $=-6.800 \mathrm{e}-03+($ ctemp -273.15$) * 9.70 \mathrm{e}-06$

120

continue

return

end

\title{
FRAPTRAN 1.4: CTHEXP
}

\author{
* deck cthexp \\ subroutine cthexp (ctemp, cathex, cdthex, icm) \\ implicit real (a-h,o-z) \\ C \\ cthexp calculates axial and diametral thermal expansion of \\ zircaloy lwr cladding. \\ cathex $=$ output axial thermal expansion of zircaloy $(\mathrm{m} / \mathrm{m})$ \\ cdthex $=$ output diametral thermal expansion of zircaloy $(\mathrm{m} / \mathrm{m})$ \\ ctemp = input cladding meshpoint temperature $(\mathrm{k})$ \\ data for the correlation from room temperature to $1273 \mathrm{k}$ are \\ taken from r.l. mehan and f.w. wiesinger, "mechanical properties
}




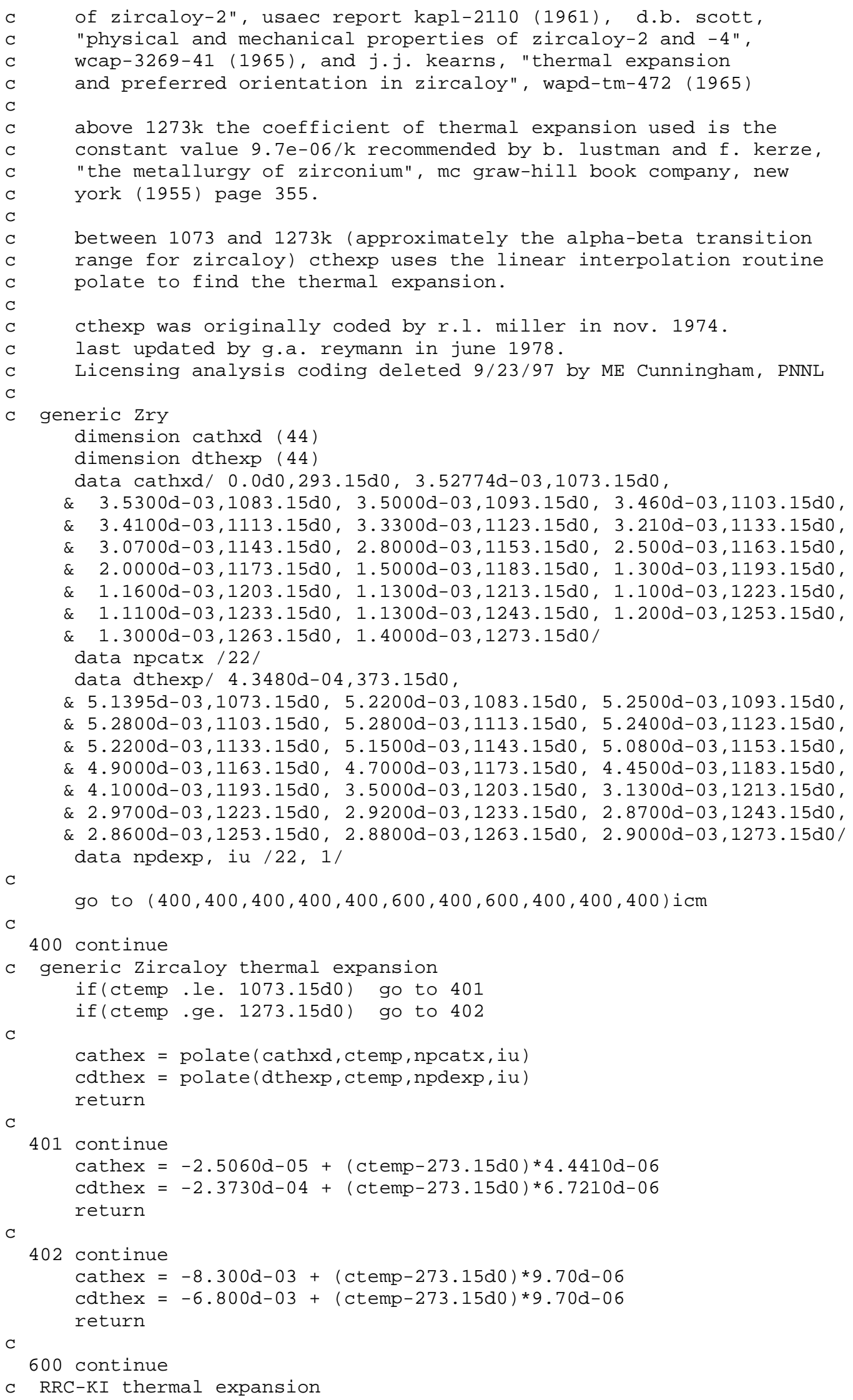

c RRC-KI thermal expansion 
C

if(ctemp .ge. 2133.d0) then

cathex $=1.0582459 \mathrm{~d}-2$

cdthex $=1.3133600 \mathrm{~d}-2$

C

else if(ctemp.gt.1153.d0) then

cathex $=1.076459 d-3+9.7 d-6^{*}($ ctemp $-1153 . d \odot)$

cdthex $=3.627600 d-3+9.7 d-6^{*}($ ctemp $-1153 . d \odot)$

C

else if(ctemp.gt. 883.d0)then

cathex $=3.0465577 d-3+2.312 d-8^{*}($ ctemp $-883 . d \odot)$

\& $\quad-7.358 d-8^{*}($ ctemp $-883 . d \odot) * * 2+1.7211 d-10^{*}($ ctemp $-883 . d 0) * * 3$ cdthex $=5.5977000 \mathrm{~d}-3+2.312 \mathrm{~d}-8 *($ ctemp $-883 . \mathrm{d} \odot)$

\& $\quad-7.358 d-8 *($ ctemp $-883 . d 0) * * 2+1.7211 d-10 *($ ctemp $-883 . d 0) * * 3$

C

else if(ctemp.gt. 573.d 0 ) then cathe $=\odot .13725577 d-2+5.4 d-6 *($ ctemp $-573 . d \odot)$

cdthe $x=\odot .3336985 d-8{ }^{*}$ ctemp $^{*}{ }^{*} 2+5.65390 d-6{ }^{*}$ ctemp $-\odot .199649865 d-2$

C

else

cathe $=0.1338985 d-8 *$ ctemp $*{ }^{*} 2+3.85875 d-6{ }^{*}$ ctemp $-0.127813365 d-2$

cdthex $=0.3336985 d-8{ }^{*}$ ctemp $^{*}{ }^{*} 2+5.65390 d-6{ }^{*}$ ctemp $-\odot .199649865 d-2$ endif

C

return

end

\section{A.2.6 Cladding Elastic Modulus (CELMOD) and Shear Modulus (CSHEAR)}

\section{FRAPCON-3.4: CELMOD}

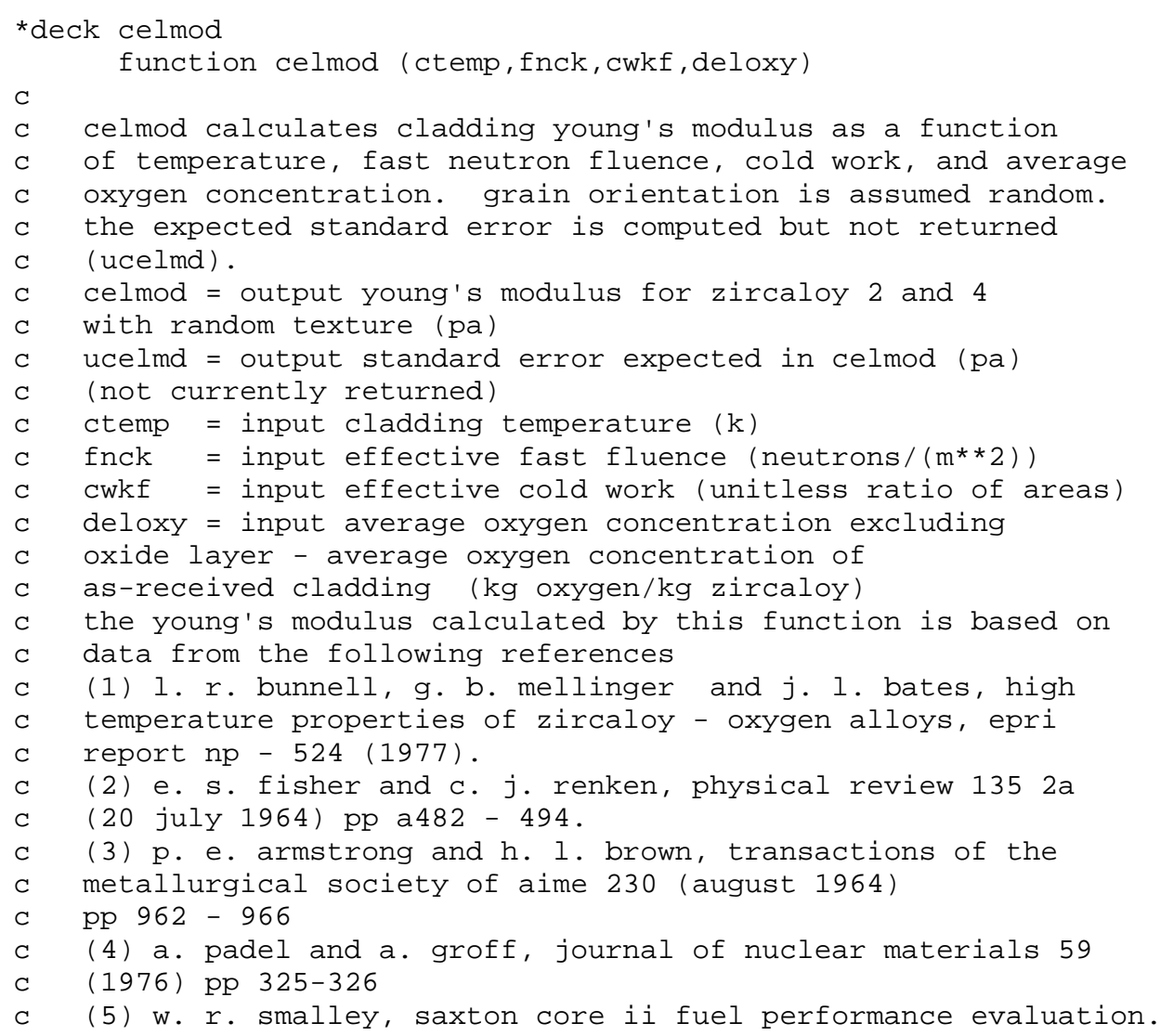




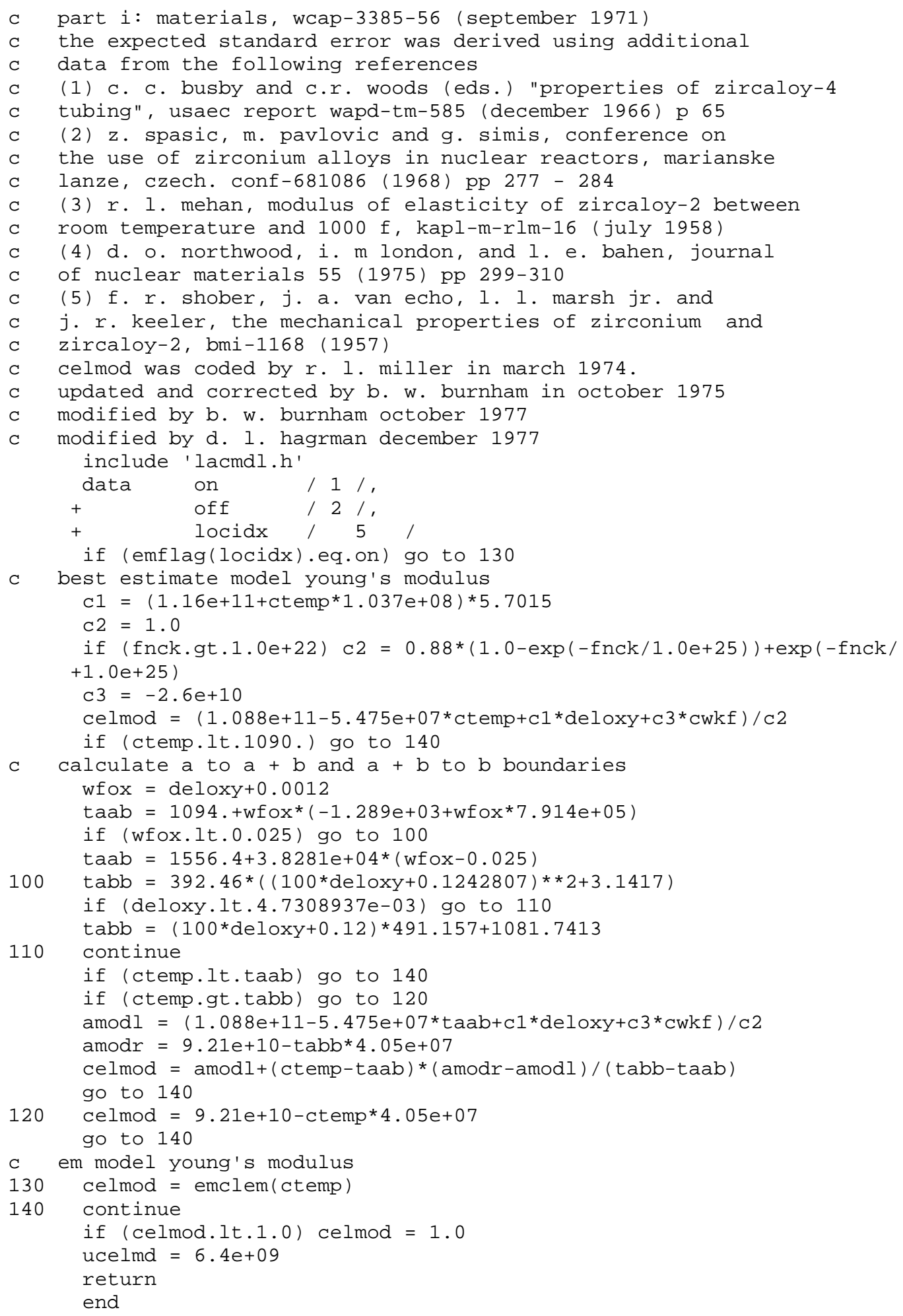

\section{FRAPTRAN1.4: CELMOD}

* deck celmod

c

function celmod (ctemp, fnck, cwkf, deloxy, icm) 
C

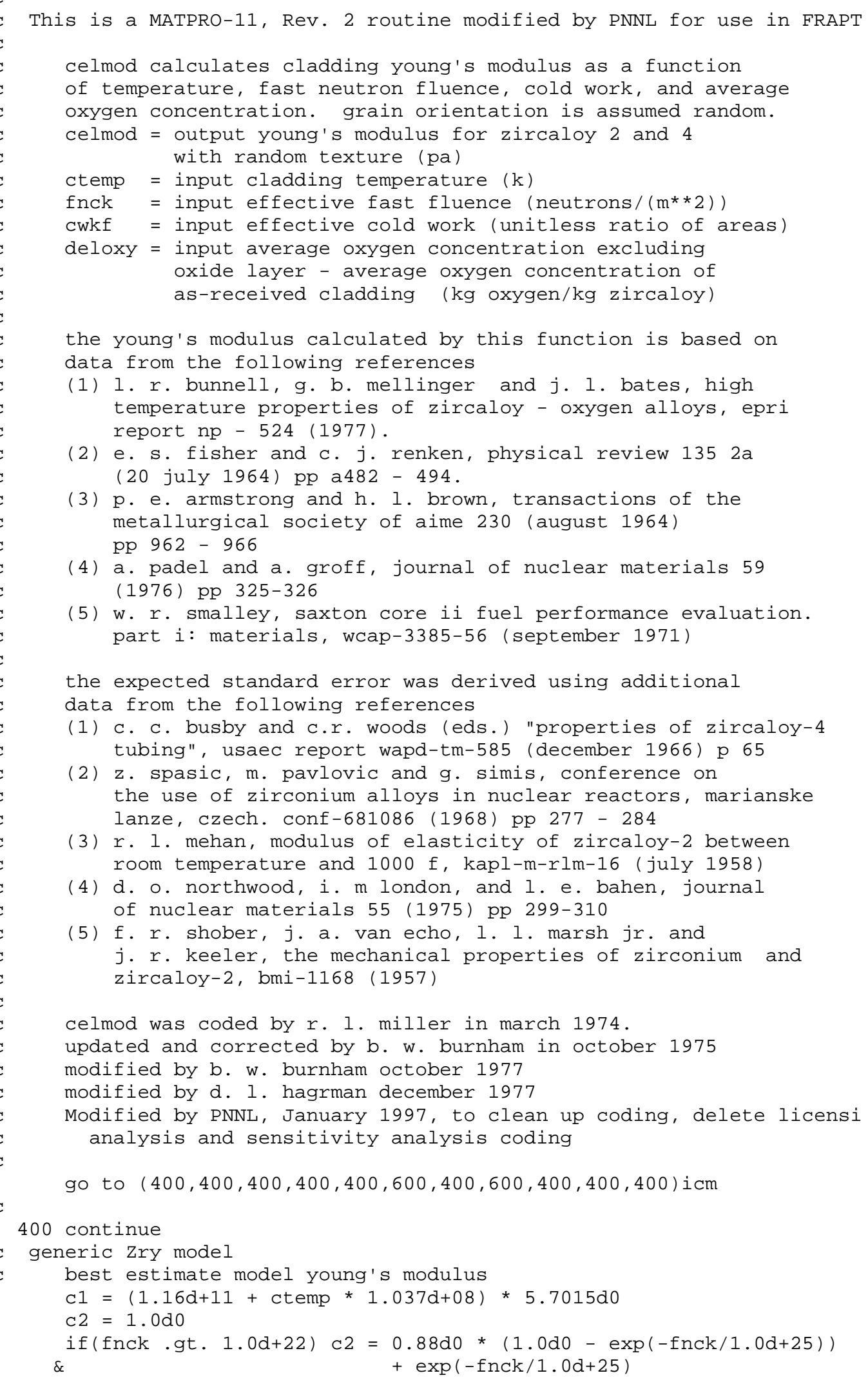




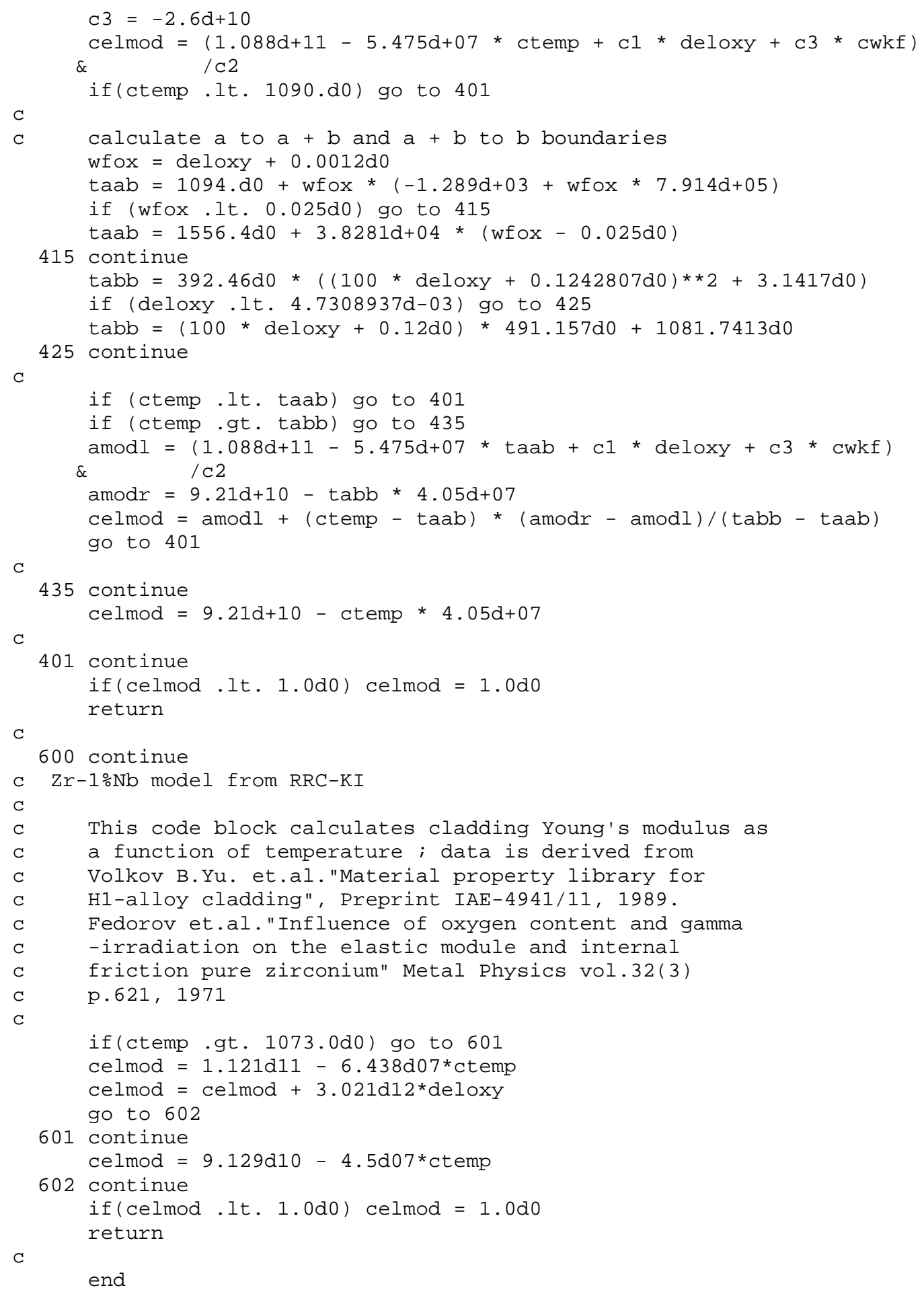

\section{FRAPCON-3.4: CSHEAR}

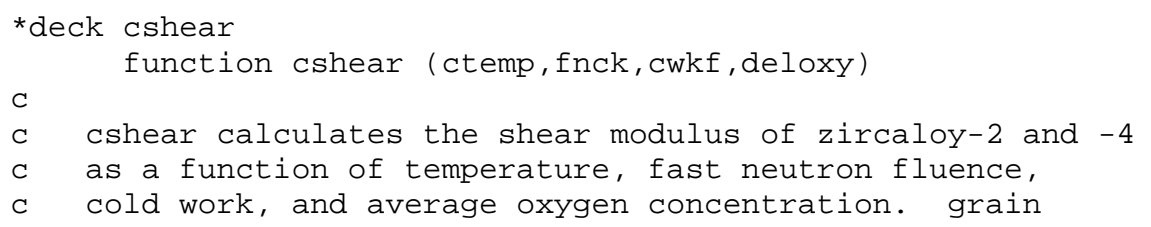




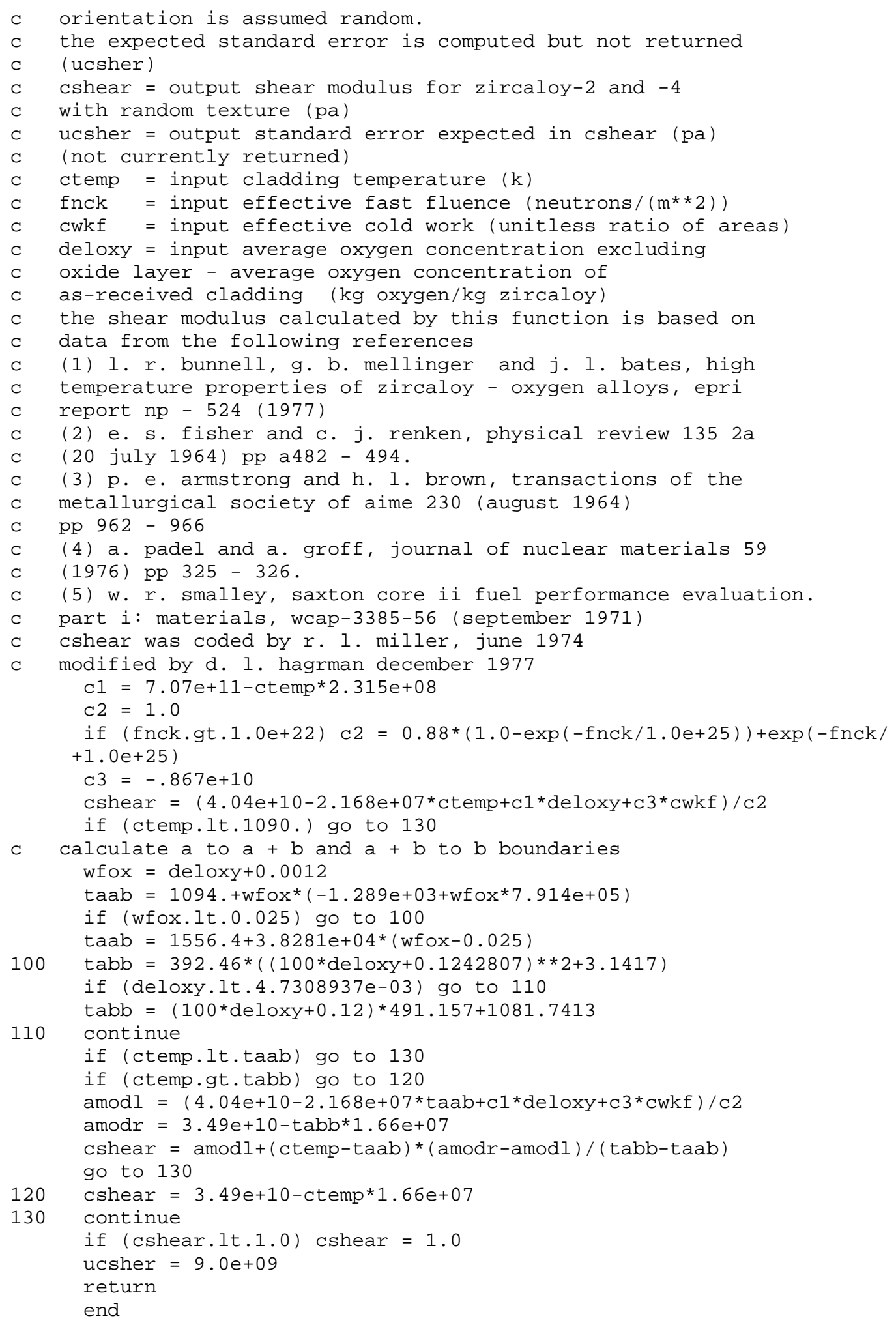

\section{FRAPTRAN 1.4: CSHEAR}

* deck cshear

c 


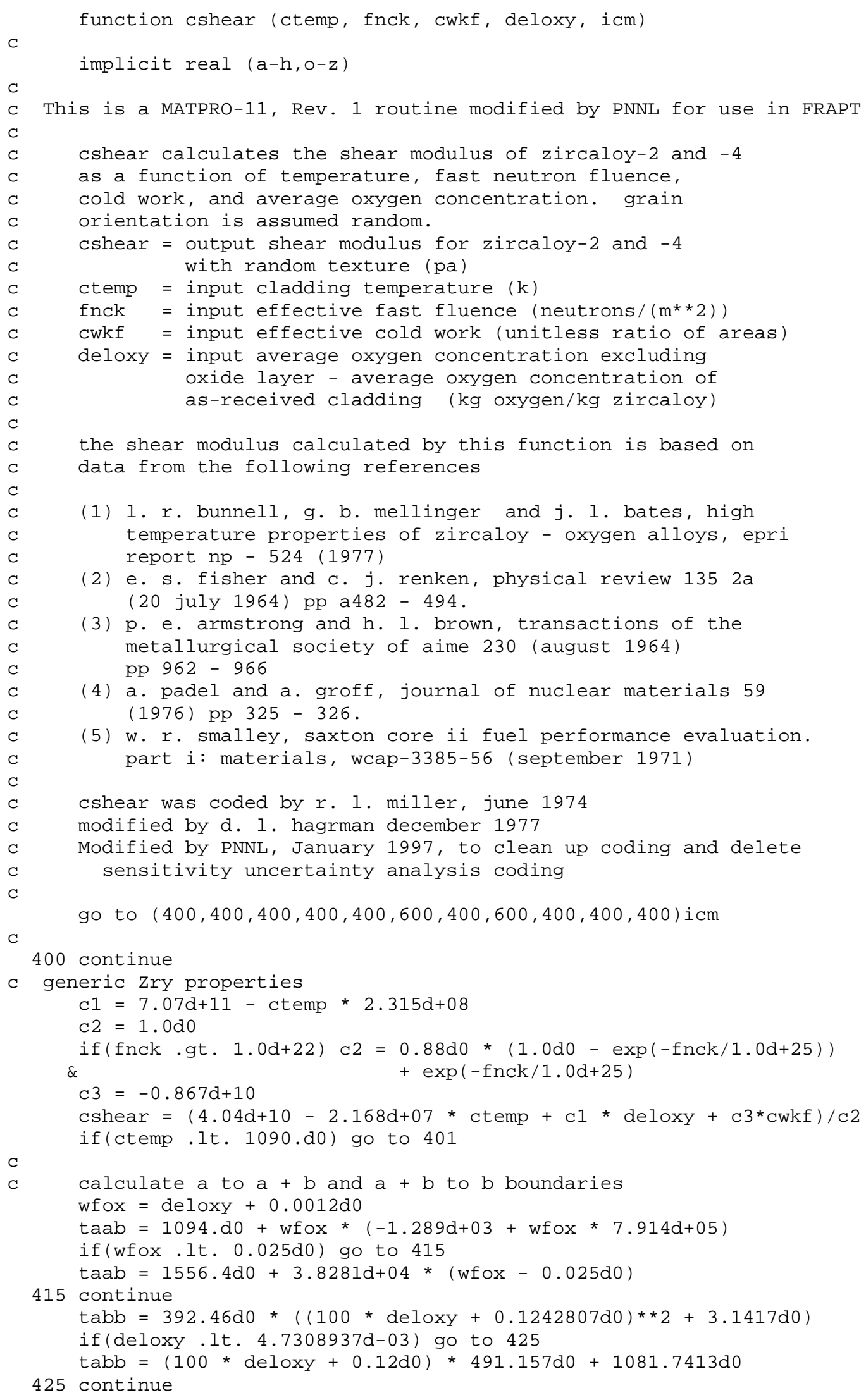




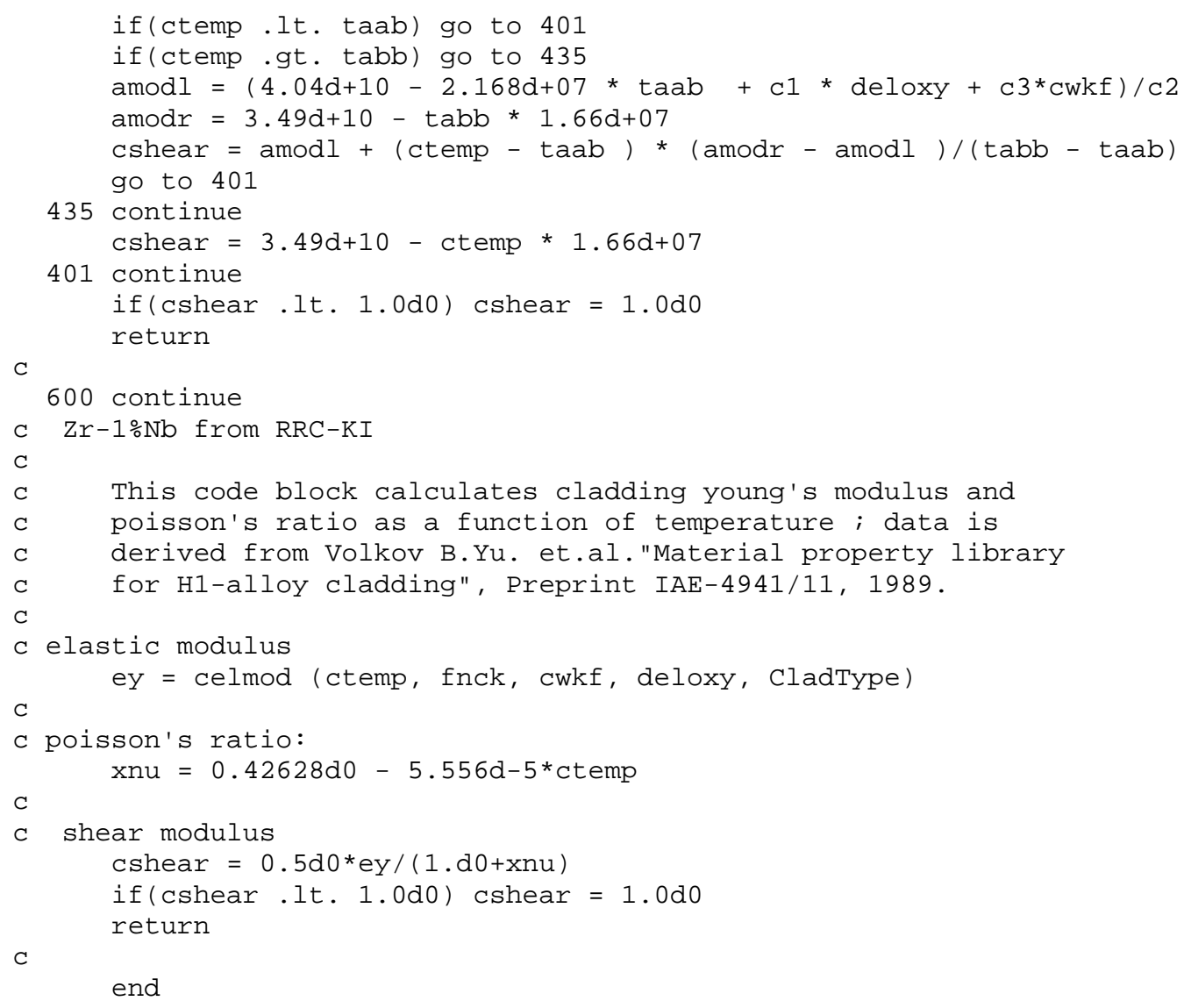

\section{A.2.7 Cladding Axial Growth (CAGROW)}

\section{FRAPCON-3.4: CAGROW}

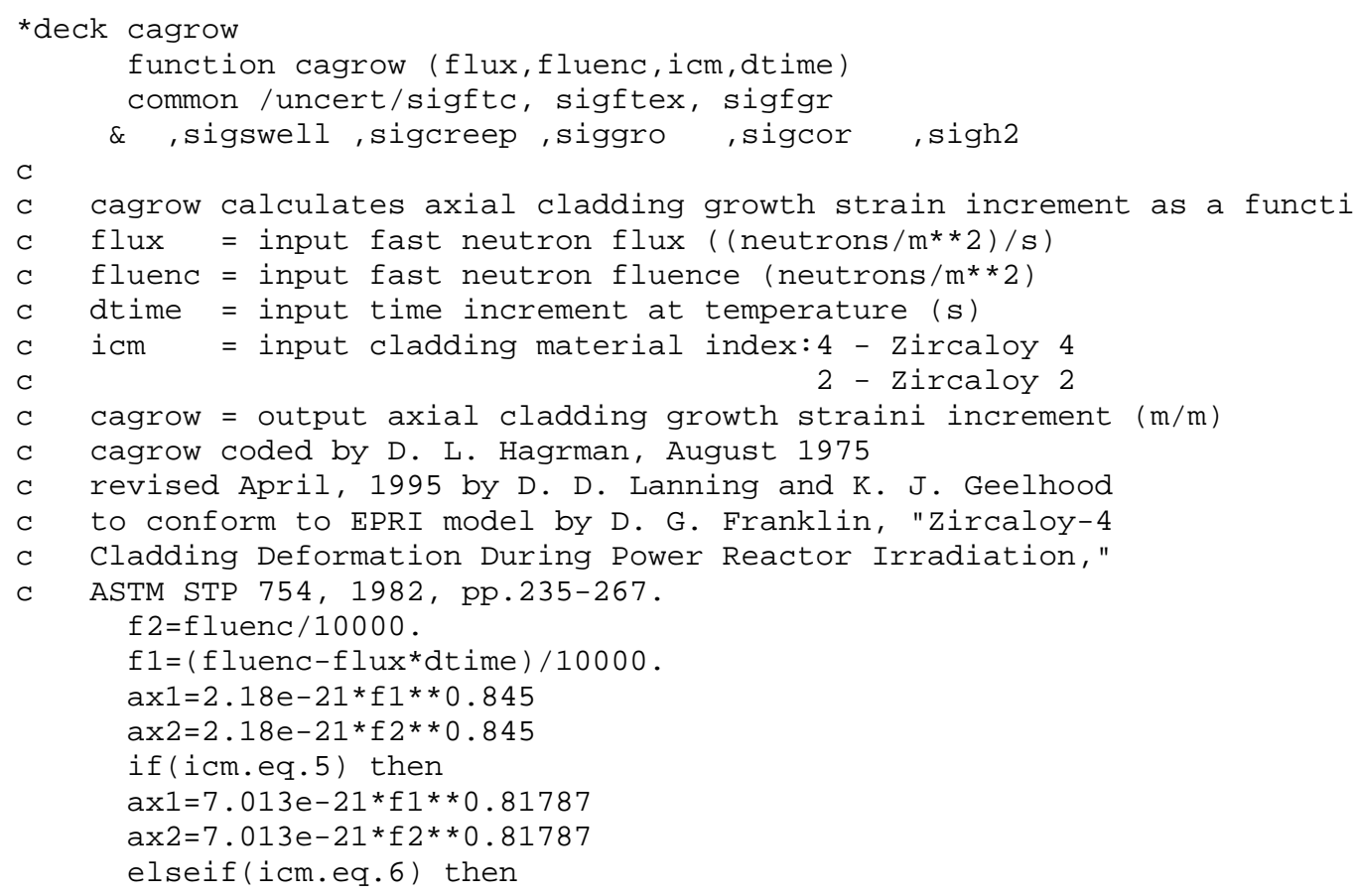




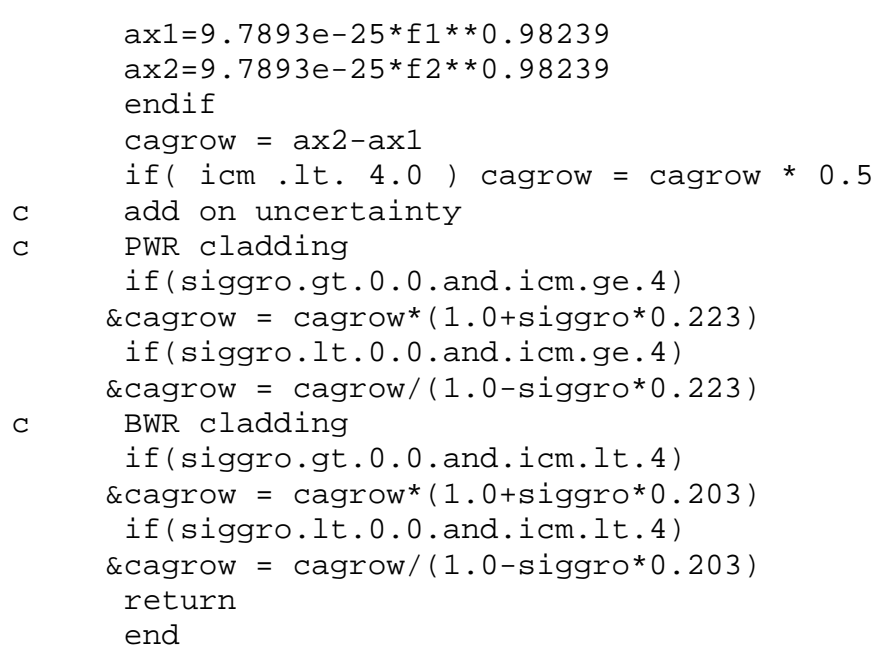

\section{A.2.8 Cladding Creep Rate (CREPR)}

\section{FRAPCON-3.4: CREPR}

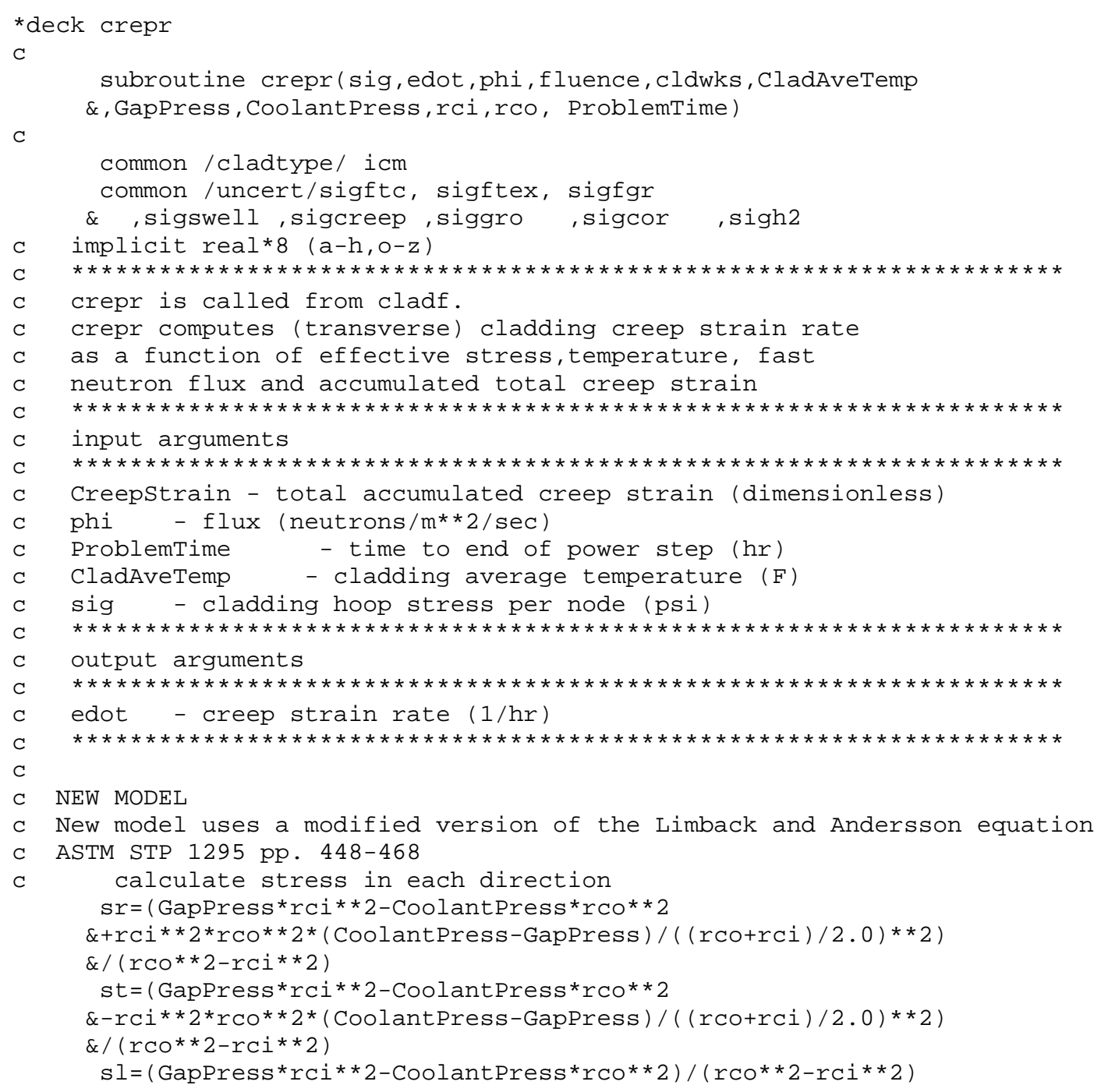


C

C

C

C

C

SRA cladding

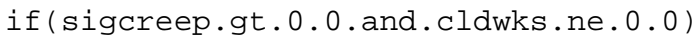

\&edot $=$ edot $^{*}(1.0+$ sigcreep $* .145)$

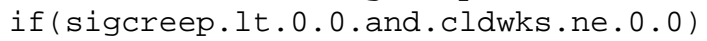

\&edot $=$ edot $/\left(1.0-\right.$ sigcreep $\left.^{*} 0.145\right)$

C

sag $=$ sig*6894.7573/1.0E6

tcak $=($ CladAveTemp+459.67)/1.8

fluencelimback = fluence $/ 10000.0$

Thermal Creep Rate

$A=1.08 \mathrm{E} 9$

if(cldwks.eq.0.0.or.icm.eq.5) $A=5.47 \mathrm{E} 8$

$=2.0$

if (cldwks.eq.0.0.or.icm.eq.5) an $=3.5$

$E=1.148 \mathrm{E} 5-59.9 *$ tcak

$Q=201000.0$

$\mathrm{R}=8.314$

Irradiation Creep Rate

COSR $=4.0985 \mathrm{E}-24$

$C \odot R X A=1.87473 E-24$

$\mathrm{C} \odot=\cos R$

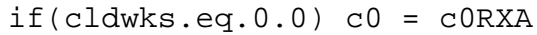

$c 1=0.85$

$c 2=1.0$

d=tcak

if (tcak.gt.625.0) tmod $=625.0$

temp $=-7.0237+0.0136 *$ tmod

Primary Creep Rate

if(ProblemTime.ge. $\odot .1)$ then

esat $=0.0216$ * (edot therm+edotirr $) * * 0.109$ *

$\&\left(2.0-\tanh \left(3.55 \mathrm{e} 4^{*}(\right.\right.$ edottherm+edotirr $\left.\left.)\right)\right) * *-2.05$

edotprimary $=$

edot therm=edot therm+edotprimary

endif

edot $=$ edottherm + edotir $r$

edot $=$ edot $* 1.1547$

if $\left(\right.$ icm.eq.6) edot $=\operatorname{edot}^{*} \odot .8$

continue

RXA cladding

$\mathrm{ai}=65 \odot . \odot *\left(1 . \odot-0.56 *\left(1 . \odot-\exp \left(-1.4 \mathrm{e}-27^{*}\right.\right.\right.$ fluenceLimback**1.3)) $)$

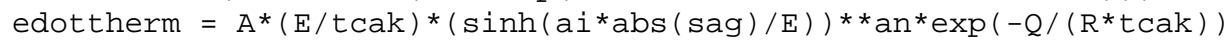

\&०. $5^{*}$ esat ${ }^{*} 52.0^{*}$ (edot therm+edotirr) ${ }^{* *} 0.5 /(($ ProblemTime $) * * 0.5)$ *

$\& \exp \left(-52.0^{*}(\right.$ edottherm+edotirr $){ }^{*} 0.5^{*}($ ProblemTime $\left.){ }^{*} \odot .5\right)$

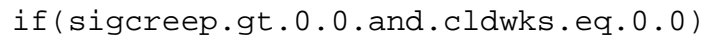

\&edot $=\operatorname{edot}^{*}(1.0+$ sigcreep $* .216)$

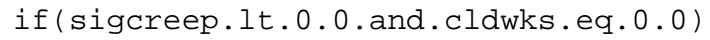

\&edot $=$ edot $/\left(1.0-\right.$ sigcreep $\left.{ }^{\star} \odot .216\right)$

return

end 


\section{A.2.9 Cladding Meyer Hardness (CMHARD)}

\section{FRAPCON-3.4: CMHARD}

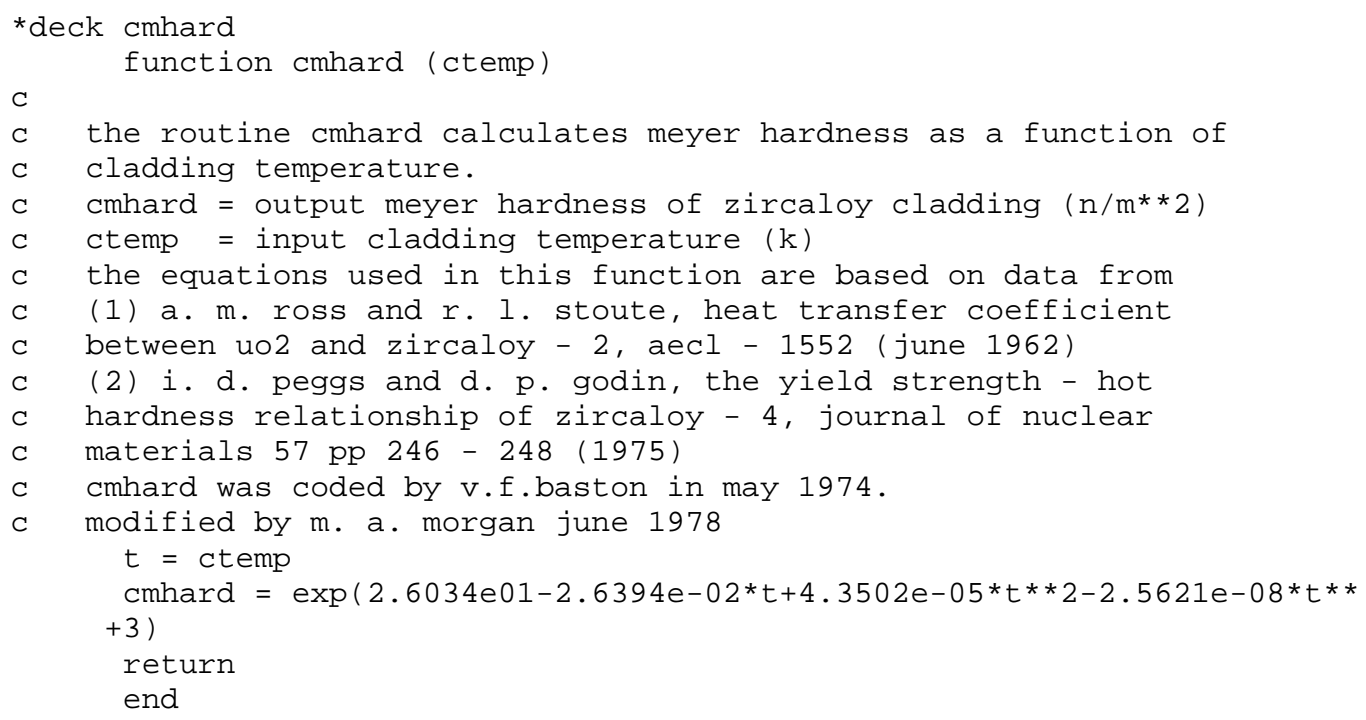

\section{FRAPTRAN 1.4: CMHARD}

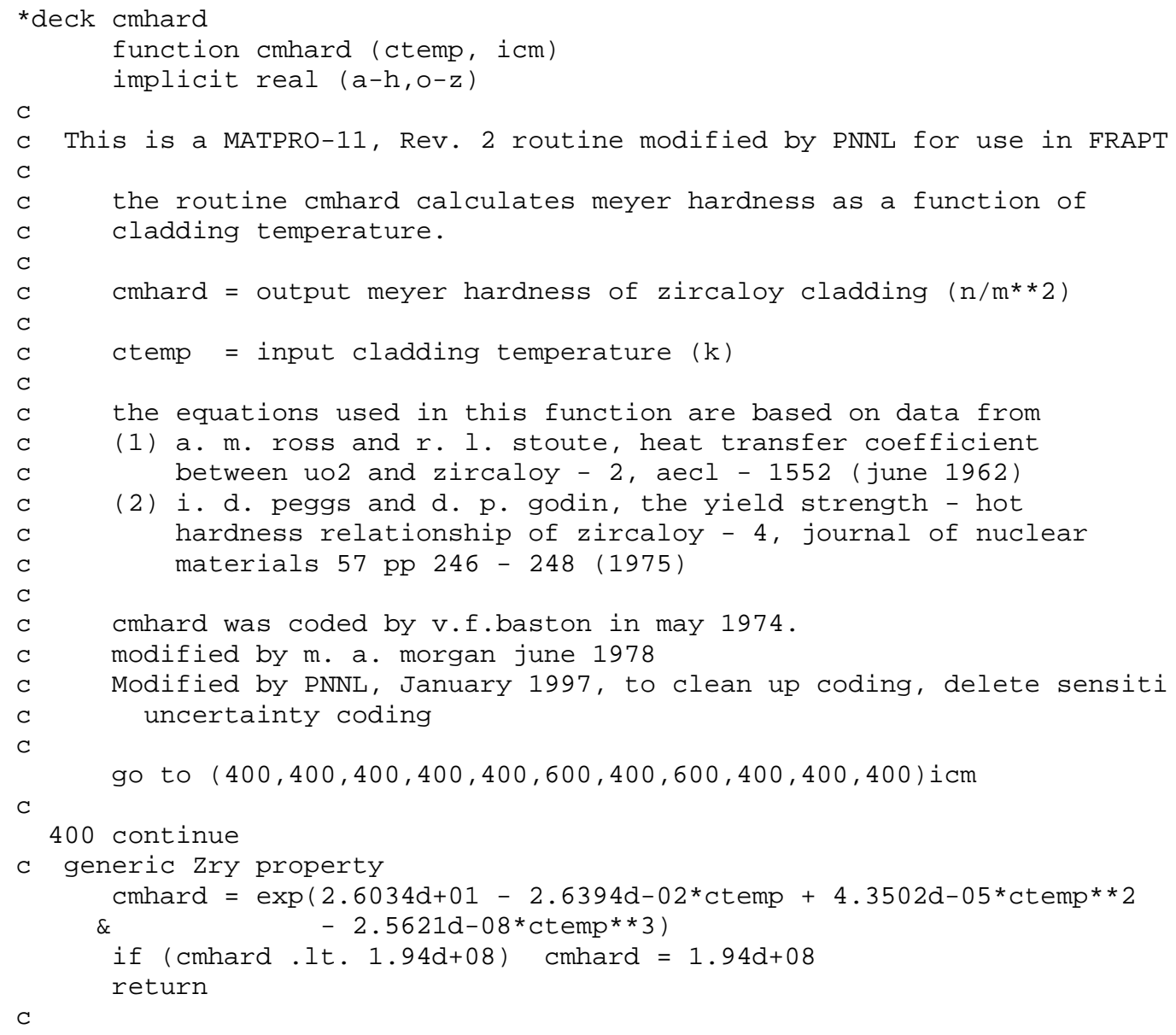




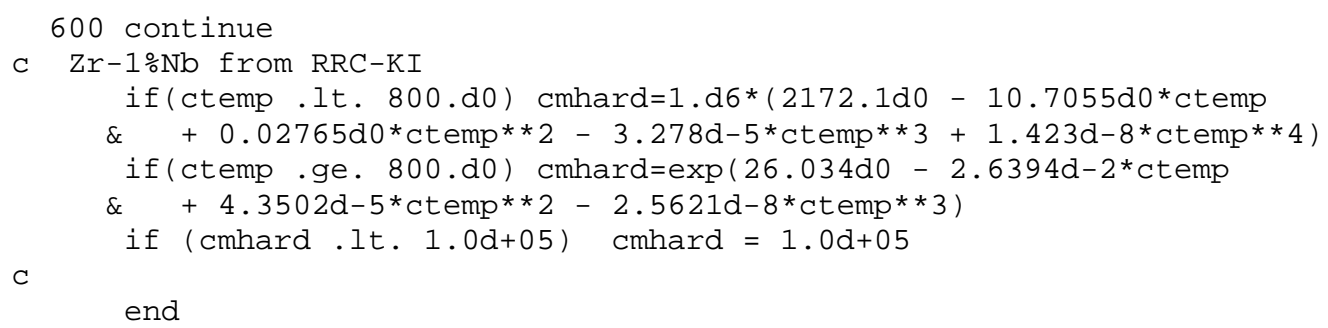

\section{A.3 Gas Material Properties}

\section{A.3.1 Gas Thermal Conductivity (GTHCON)}

\section{FRAPCON-3.4}

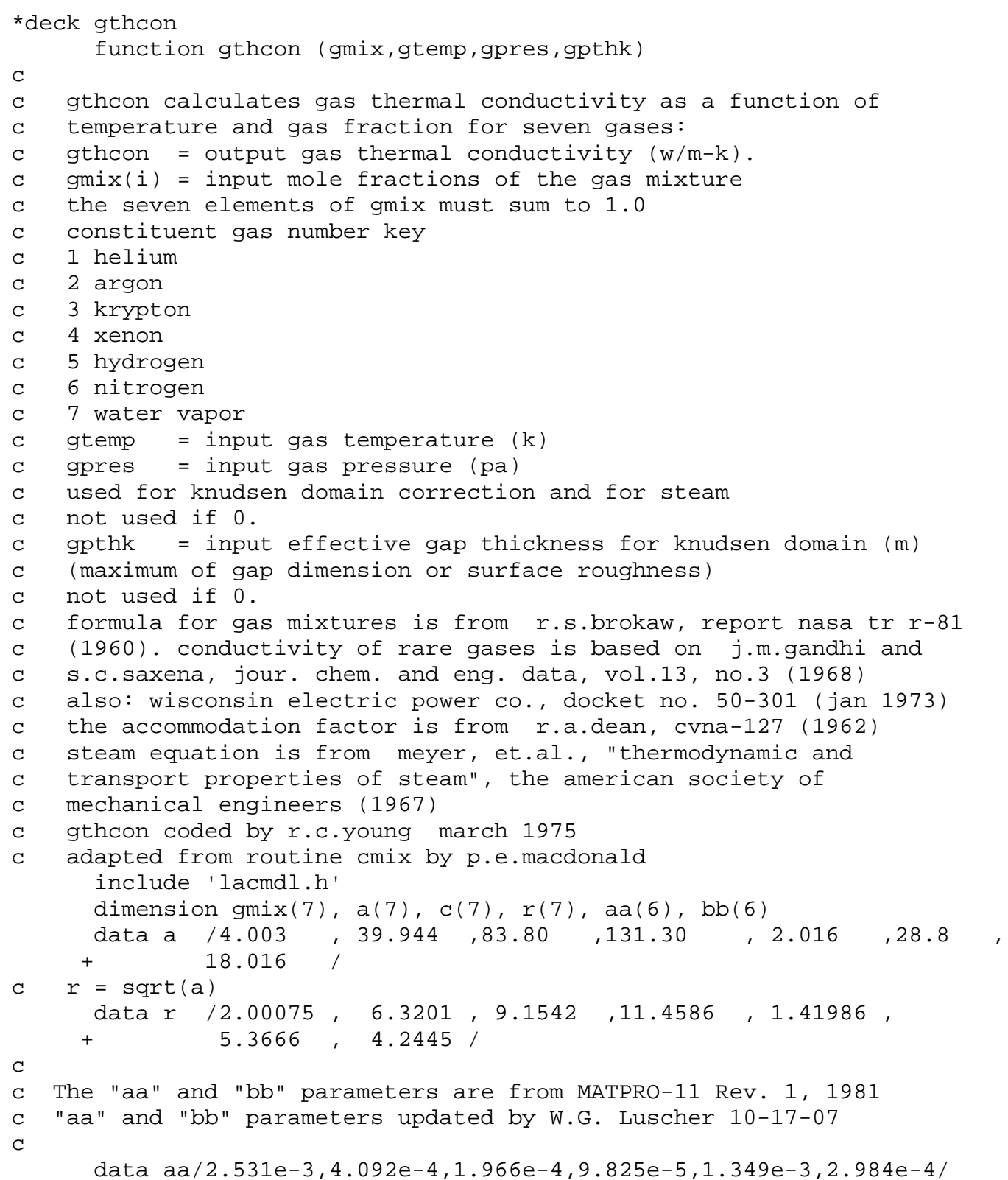




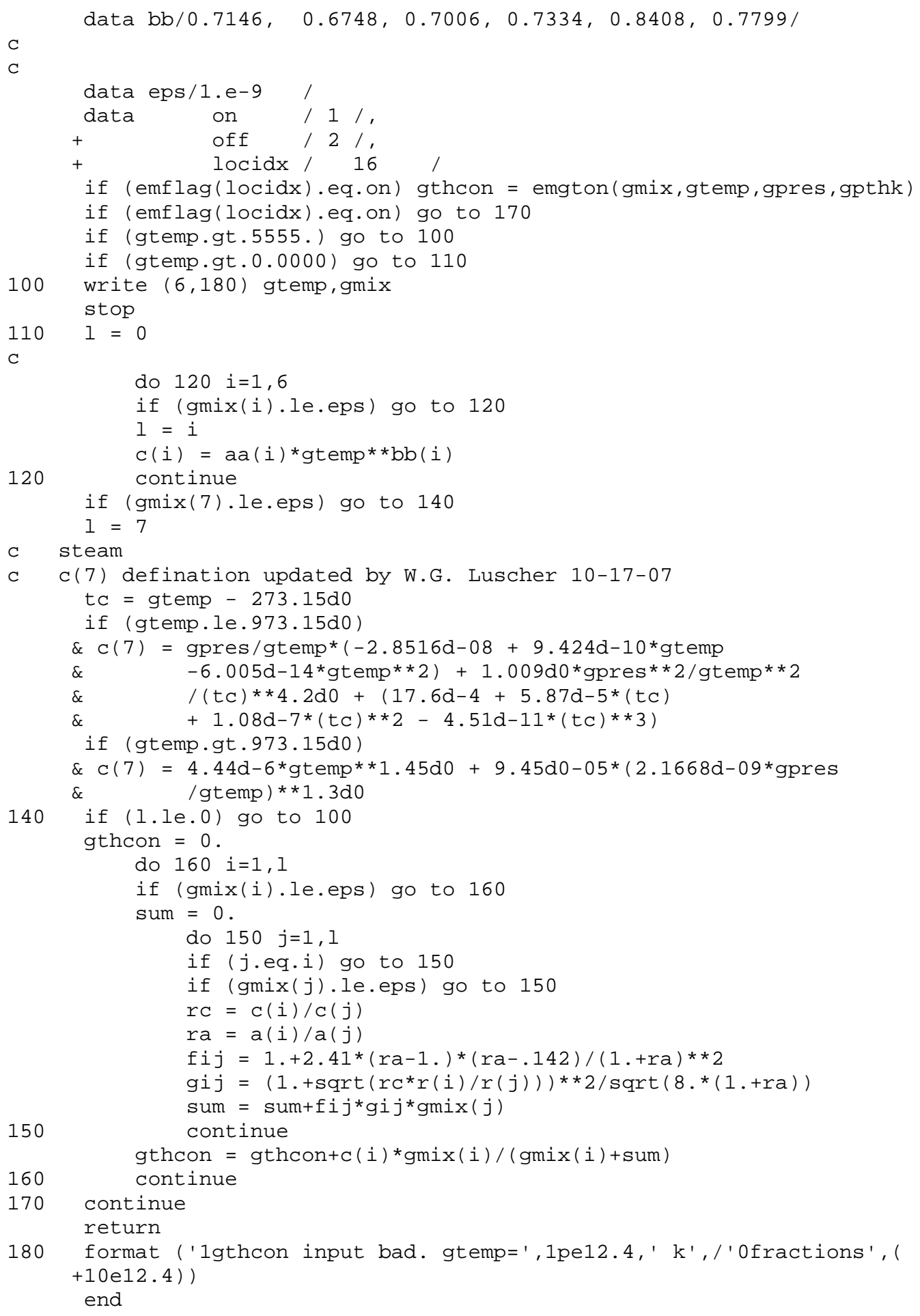

\title{
FRAPTRAN 1.4
}

\author{
*deck gthcon \\ function gthcon (gmix, gtemp, gpres, gpthk) \\ implicit real $(\mathrm{a}-\mathrm{h}, \mathrm{o}-\mathrm{z})$ \\ C \\ c gthcon calculates gas thermal conductivity as a function of
}




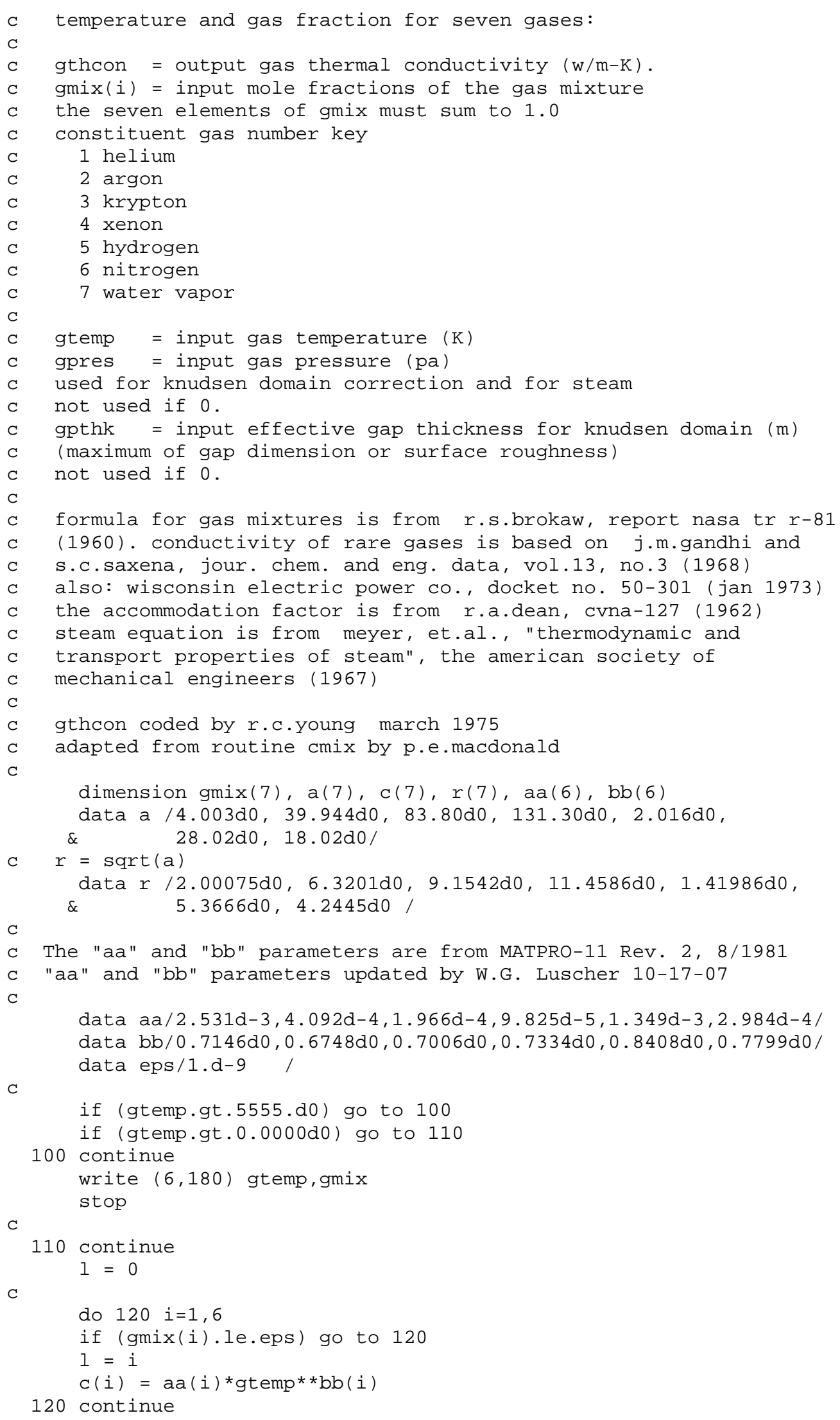




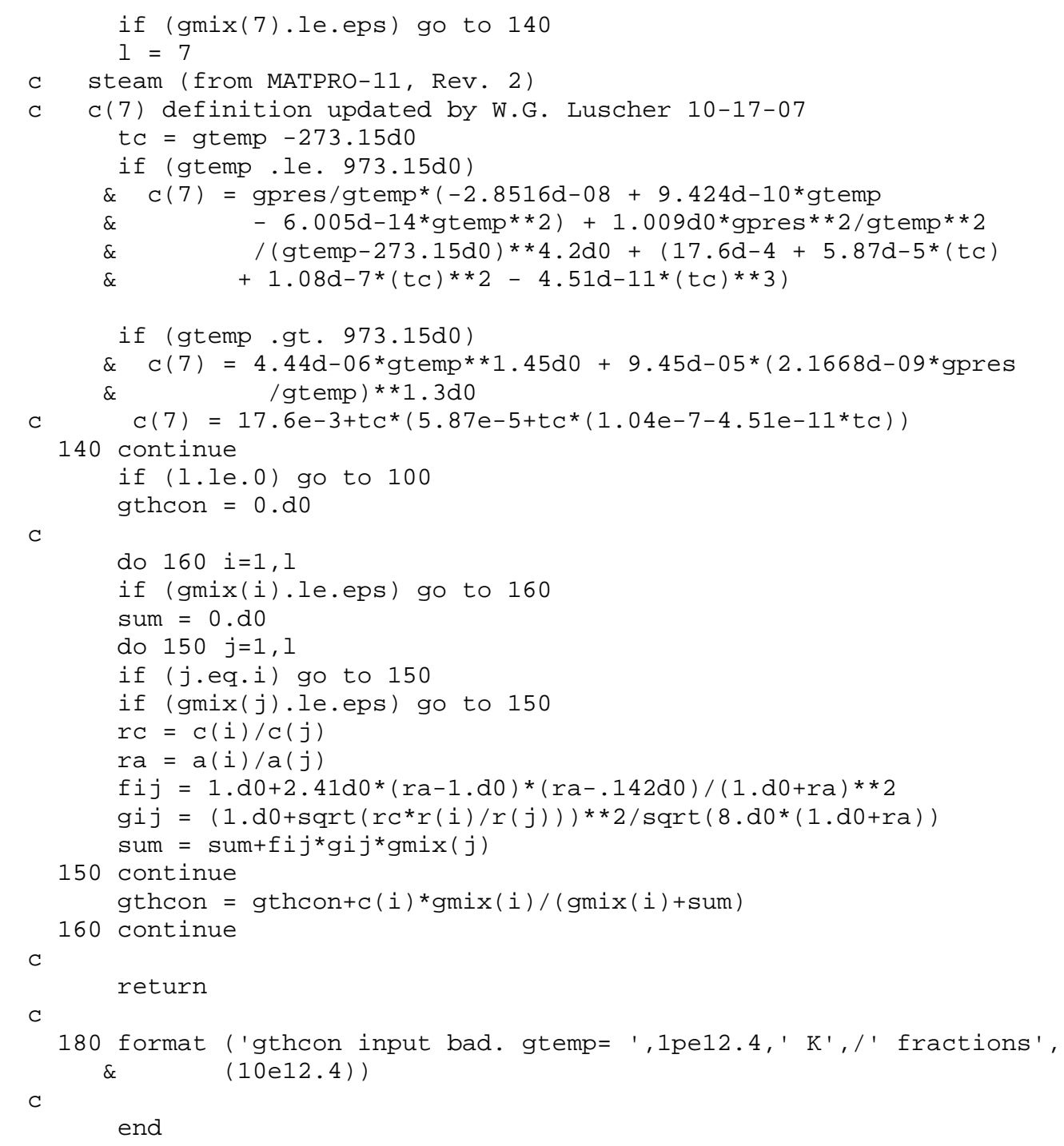

